\title{
Extended States for the Schrödinger Operator with Quasi-periodic Potential in Dimension Two
}

\author{
Yulia Karpeshina*and Roman Shterenberg
}

July 7, 2021

\begin{abstract}
We consider a Schrödinger operator $H=-\Delta+V(\vec{x})$ in dimension two with a quasi-periodic potential $V(\vec{x})$. We prove that the absolutely continuous spectrum of $H$ contains a semiaxis and there is a family of generalized eigenfunctions at every point of this semiaxis with the following properties. First, the eigenfunctions are close to plane waves $e^{i\langle\vec{\varkappa}, \vec{x}\rangle}$ at the high energy region. Second, the isoenergetic curves in the space of momenta $\vec{\varkappa}$ corresponding to these eigenfunctions have a form of slightly distorted circles with holes (Cantor type structure). A new method of multiscale analysis in the momentum space is developed to prove these results.

The result is based on the previous paper [1] on quasiperiodic polyharmonic operator $(-\Delta)^{l}+V(\vec{x}), l>1$. We address here technical complications arising in the case $l=1$. However, this text is self-contained and can be read without familiarity with [1].
\end{abstract}

\section{Contents}

1 Introduction $\quad 4$

2 Preliminary Remarks $\quad 11$

3 Step I

3.1 Operator $H^{(1)} \ldots \ldots \ldots \ldots \ldots \ldots \ldots \ldots$

3.2 Perturbation Formulas . . . . . . . . . . . . . . . . . . . . . 14

3.3 Geometric Considerations . . . . . . . . . . . . . . . . . . . . 17

3.4 Isoenergetic Surface for Operator $H^{(1)} \quad \ldots \ldots \ldots$. . . . . . . . . . 19

3.5 Preparation for Step II. Construction of the Second Nonresonant Set . . . 20

*Research partially supported by USNSF Grant DMS 1201048. 
3.5.1 Model Operator for Step II . . . . . . . . . . . . . . . . 20

3.5.2 Estimates for the Resolvent of the Model Operator . . . . . . . . 28

3.5.3 Resonant and Nonresonant Sets for Step II . . . . . . . . . . 42

4 Step II 44

4.1 Operator $H^{(2)}$. Perturbation Formulas . . . . . . . . . . . . 44

4.2 Isoenergetic Surface for Operator $H^{(2)} \ldots \ldots \ldots \ldots$

4.3 Preparation for Step III - Geometric Part. Properties of the Quasiperiodic

Lattice . . . . . . . . . . . . . . . . . . . . . 51

4.3 .1 General Lemmas . . . . . . . . . . . . . . . . . . . . . . 51

4.3.2 Lattice Points in the Nonresonant Set . . . . . . . . . . . . . . . 53

4.3.3 Lattice Points in the Resonant Set . . . . . . . . . . . . . . 54

4.4 Preparation for Step III - Analytic Part . . . . . . . . . . . . . 66

4.4 .1 Model Operator for Step III . . . . . . . . . . . . . . . . . 66

4.4.2 Resonant and Nonresonant Sets for Step III . . . . . . . . . . . 75

5 Step III 76

5.1 Operator $H^{(3)}$. Perturbation Formulas . . . . . . . . . . . 77

5.2 Isoenergetic Surface for Operator $H^{(3)} \ldots \ldots \ldots$. . . . . . . . . 84

5.3 Preparation for Step IV . . . . . . . . . . . . . . . . . 85

5.3.1 Properties of the Quasiperiodic Lattice. Continuation . . . . . . . 85

5.3 .2 Model Operator for Step IV . . . . . . . . . . . . . . . . . 93

5.3.3 Resonant and Nonresonant Sets for Step IV . . . . . . . . . . . . 99

6 STEP IV 100

6.1 Operator $H^{(4)}$. Perturbation Formulas . . . . . . . . . . . . . . 100

6.2 Isoenergetic Surface for Operator $H^{(4)} \ldots \ldots \ldots$

7 Induction 103

7.1 Inductive formulas for $r_{n} \ldots \ldots \ldots \ldots \ldots \ldots$

7.2 Preparation for Step $n+1, n \geq 4 \ldots \ldots \ldots$

7.2.1 Properties of the Quasiperiodic Lattice. Induction . . . . . . . . . 103

7.2 .2 Model Operator for Step $n+1 \ldots \ldots \ldots \ldots$

7.2.3 Resonant and Nonresonant Sets for Step $n+1 \ldots \ldots \ldots$

7.3 Operator $H^{(n+1)}$. Perturbation Formulas . . . . . . . . . . . . . 112

7.4 Isoenergetic Surface for Operator $H^{(n+1)} \ldots \ldots \ldots \ldots$

8 Isoenergetic Sets. Generalized Eigenfunctions of $H \quad \mathbf{1 1 4}$

8.1 Construction of Limit-Isoenergetic Set . . . . . . . . . . . . . . . . 114

8.2 Generalized Eigenfunctions of $H \ldots \ldots \ldots \ldots \ldots \ldots$ 
9 Proof of Absolute Continuity of the Spectrum 119

9.1 Operators $E_{n}\left(\mathcal{G}_{n}^{\prime}\right), \mathcal{G}_{n}^{\prime} \subset \mathcal{G}_{n} \ldots \ldots \ldots \ldots \ldots \ldots \ldots$

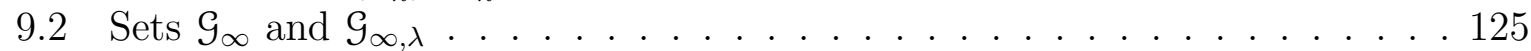

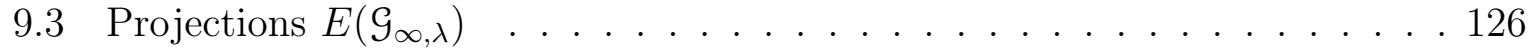

9.4 Proof of Absolute Continuity . . . . . . . . . . . . . . . . . . 128

10 Appendices $\quad \mathbf{1 2 8}$

10.1 Appendix 1. Proof of Lemma $3.12 \ldots \ldots \ldots \ldots \ldots \ldots$

10.2 Appendix 2. Proof of Lemma $3.13 \ldots \ldots \ldots$

10.3 Appendix $3 \ldots \ldots \ldots$

10.4 Appendix $4 \ldots \ldots \ldots \ldots$

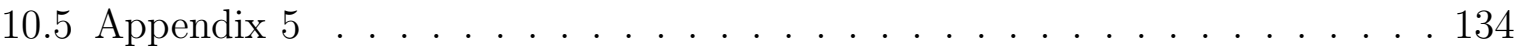

10.6 Appendix $6 \ldots \ldots \ldots \ldots \ldots$

10.7 Appendix $7 \ldots \ldots \ldots$

10.8 Appendix 8. On Application of Bezout Theorem . . . . . . . . . . . . . . 139

10.9 Appendix 9. On the Proof of Geometric Lemmas Allowing to Deal with

Clusters instead of Boxes . . . . . . . . . . . . . . . . . 140

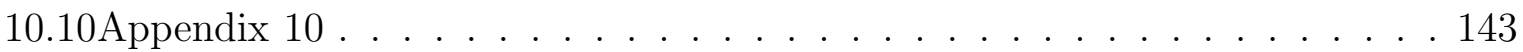

11 List of the main notations 


\section{Introduction}

We study an operator

$$
H=-\Delta+V(\vec{x})
$$

in dimension two, $V(\vec{x})$ being a quasi-periodic potential:

$$
V=\sum_{\mathbf{s}_{1}, \mathbf{s}_{2} \in \mathbb{Z}^{2}, \mathbf{s}_{1}+\alpha \mathbf{s}_{2} \in \mathcal{S}_{Q}} V_{\mathbf{s}_{1}, \mathbf{s}_{2}} e^{2 \pi i\left\langle\mathbf{s}_{1}+\alpha \mathbf{s}_{2}, \vec{x}\right\rangle},
$$

where $\alpha$ is irrational number and $\mathcal{S}_{Q}$ is a finite set. To simplify the construction we put some additional conditions on $\alpha$ and $\mathcal{S}_{Q}$, see the beginning of Section 2 .

The one-dimensional situation $d=1$ is thoroughly investigated in discrete and continuum settings, see e.g. [2]-[13] and references there. It is known that a one-dimensional quasi-periodic Schrödinger operator demonstrates spectral and transport properties which are not close to those of a periodic operator. The spectrum of the quasi-periodic operator is, as a rule, a Cantor set, while in the periodic case, it has a band structure. In the periodic case the spectrum is absolutely continuous, while in the quasi-periodic case, it can have any nature: absolutely continuous, singular continuous and pure point. The transition between different types of spectrum can happen even with a small change of a coefficient in a quasi-periodic operator (see [9], [13]-[15]). The mechanism of the difference in spectral behavior between periodic and quasi-periodic cases can be explained by a phenomenon which is known as resonance tunneling in quantum mechanics. It is associated with small denominators appearing in formal series of perturbation theory. Since the spectrum of the one-dimensional Laplacian is thin (multiplicity 2), resonance tunneling can produce an effect strong enough to destroy the spectrum. If a potential is periodic, then resonance tunneling produces gaps in the spectrum near the points $\lambda_{n}=(\pi n / a)^{2}$, $n \in \mathbb{Z}, a$ being the period of the potential. If the potential is quasi-periodic, then it can be thought as a sort of combination of infinite number of periodic potentials, each of them producing gaps near its own $\lambda_{n}$-s. Since the set of all $\lambda_{n}$-s can be dense, the number of points surrounded by gaps can be dense too. Thus, the spectrum gets a Cantor like structure. The properties of the operator in the high energy region for the continuum case $d=1$ are studied in [2]-[5], [8]. The KAM method is used to prove absolute continuity of the spectrum and existence of quasiperiodic solutions at high energies.

There are important results on the density of states, spectrum, localization concerning the quasi-periodic operators in $\mathbb{Z}^{d}$ and, partially, in $\mathbb{R}^{d}, d>1$, e.g. [16]-[26]. However, it is still much less known about (1) than about its one-dimensional analog. The properties of the spectrum in the high energy region, existence of extended states and quantum transport are still the wide open problems in the multidimensional case. It is worth noticing here the discrete results [44] and [45] which show that for discrete model the spectrum is pure point for a wide class of limit-periodic potentials and thus, no absolutely continuous spectrum is present. 
Here we study properties of the spectrum and eigenfunctions of (11) in the high energy region. We prove the following results for the case $d=2$. This is the extension of the analogous results proven by the authors [1] for the quasiperiodic operator $(-\Delta)^{l}+V$, $l>1, d=2$.

1. The spectrum of the operator (1) contains a semiaxis.

This is a generalization of a renown Bethe-Sommerfeld conjecture, which states that in the case of a periodic potential and $d \geq 2$, the spectrum of (1) contains a semiaxis. There is a variety of proofs for the periodic case, the earliest one is [27], for the most general case see [28]. For a limit-periodic periodic potential, being periodic in one direction, the conjecture is proved in [29]. For a general case of limit-periodic potential the conjecture is proven in [30]- 32 .

2. There are generalized eigenfunctions $\Psi_{\infty}(\vec{\varkappa}, \vec{x})$, corresponding to the semi-axis, which are close to plane waves: for every $\vec{\varkappa}$ in an extensive subset $\mathcal{G}_{\infty}$ of $\mathbb{R}^{2}$ (see (6) below $)$, there is a solution $\Psi_{\infty}(\vec{\varkappa}, \vec{x})$ of the equation $H \Psi_{\infty}=\lambda_{\infty} \Psi_{\infty}$ which can be described by the formula:

$$
\begin{gathered}
\Psi_{\infty}(\vec{\varkappa}, \vec{x})=e^{i\langle\vec{\varkappa}, \vec{x}\rangle}\left(1+u_{\infty}(\vec{\varkappa}, \vec{x})\right), \\
\left\|u_{\infty}\right\|_{L_{\infty}\left(\mathbb{R}^{2}\right)} \underset{|\vec{\imath}| \rightarrow \infty}{=} O\left(|\vec{\varkappa}|^{-\gamma_{1}}\right), \quad \gamma_{1}>0,
\end{gathered}
$$

where $u_{\infty}(\vec{\varkappa}, \vec{x})$ is a quasi-periodic function, namely a point-wise convergent series of exponentials $e^{2 \pi i\langle\mathbf{n}+\alpha \mathbf{m}, \vec{x}\rangle}, \mathbf{n}, \mathbf{m} \in \mathbb{Z}^{2}$. The eigenvalue $\lambda_{\infty}(\overrightarrow{\mathcal{L}})$, corresponding to $\Psi_{\infty}(\vec{\varkappa}, \vec{x})$, is close to $|\vec{\varkappa}|^{2}$ :

$$
\lambda_{\infty}(\vec{\varkappa}) \underset{|\vec{x}| \rightarrow \infty}{=}|\vec{\varkappa}|^{2}+O\left(|\vec{\varkappa}|^{-\gamma_{2}}\right), \quad \gamma_{2}>0 .
$$

The "non-resonant" set $\mathcal{G}_{\infty}$ of vectors $\vec{\varkappa}$, for which (3) - (5) hold, is a Cantor type set: $\mathcal{G}_{\infty}=\cap_{n=1}^{\infty} \mathcal{G}_{n}$, where $\left\{\mathcal{G}_{n}\right\}_{n=1}^{\infty}$ is a decreasing sequence of sets in $\mathbb{R}^{2}$. Each $\mathcal{G}_{n}$ has a finite number of holes in each bounded region. More and more holes appear when $n$ increases, however holes added at each step are of smaller and smaller size. The set $\mathcal{G}_{\infty}$ is extensive in the sense that it satisfies the estimate:

$$
\left|\mathcal{G}_{\infty} \cap \mathbf{B}_{\mathbf{R}}\right| \underset{R \rightarrow \infty}{=}\left|\mathbf{B}_{\mathbf{R}}\right|\left(1+O\left(R^{-\gamma_{3}}\right)\right), \quad \gamma_{3}>0
$$

where $\mathbf{B}_{\mathbf{R}}$ is the disk of radius $R$ centered at the origin, $|\cdot|$ is the Lebesgue measure in $\mathbb{R}^{2}$.

3. The set $\mathcal{D}_{\infty}(\lambda)$, defined as a level (isoenergetic) set for $\lambda_{\infty}(\vec{\varkappa})$,

$$
\mathcal{D}_{\infty}(\lambda)=\left\{\vec{\varkappa} \in \mathcal{G}_{\infty}: \lambda_{\infty}(\vec{\varkappa})=\lambda\right\}
$$


is proven to be a slightly distorted circle with infinite number of holes. It can be described by the formula:

$$
\mathcal{D}_{\infty}(\lambda)=\left\{\vec{\varkappa}: \vec{\varkappa}=\varkappa_{\infty}(\lambda, \vec{\nu}) \vec{\nu}, \vec{\nu} \in \mathcal{B}_{\infty}(\lambda)\right\}
$$

where $\mathcal{B}_{\infty}(\lambda)$ is a subset of the unit circle $S_{1}$. The set $\mathcal{B}_{\infty}(\lambda)$ can be interpreted as the set of possible directions of propagation for almost plane waves (3). The set $\mathcal{B}_{\infty}(\lambda)$ has a Cantor type structure and an asymptotically full measure on $S_{1}$ as $\lambda \rightarrow \infty$ :

$$
L\left(\mathcal{B}_{\infty}(\lambda)\right) \underset{\lambda \rightarrow \infty}{=} 2 \pi+O\left(\lambda^{-\gamma_{4}}\right), \quad \gamma_{4}>0,
$$

here and below $L(\cdot)$ is a length of a curve. The value $\varkappa_{\infty}(\lambda, \vec{\nu})$ in (7) is the "radius" of $\mathcal{D}_{\infty}(\lambda)$ in a direction $\vec{\nu}$. The function $\varkappa_{\infty}(\lambda, \vec{\nu})-\lambda^{1 / 2}$ describes the deviation of $\mathcal{D}_{\infty}(\lambda)$ from the perfect circle of the radius $\lambda^{1 / 2}$. It is proven that the deviation is asymptotically small:

$$
\varkappa_{\infty}(\lambda, \vec{\nu}) \underset{\lambda \rightarrow \infty}{=} \lambda^{1 / 2}+O\left(\lambda^{-\gamma_{5}}\right), \quad \gamma_{5}>0
$$

4. The branch of the spectrum of the operator (1) corresponding to the generalized eigenfunctions $\Psi_{\infty}(\vec{\varkappa}, \vec{x})$ is absolutely continuous.

To prove the results listed above we suggest a method which can be described as multiscale analysis in the space of momenta. This is a development of a method applied in [1] for operator $(-\Delta)^{l}+V, l>1, d=2$. The present case $l=1$ has technical complications, comparing with $l>1$. These complications are in the first steps of an approximation procedure. They are related to the fact, that $V$ is a stronger perturbation with respect to $-\Delta$ than with respect to $(-\Delta)^{l}, l>1$. The method is also related to that developed in [30]-32] for limit-periodic potentials. The essential difference is that in [30] -32 there was constructed a modification of KAM method, where the space variable $\vec{x}$ still plays some role (e.g. in the uniform in $\vec{x}$ approximation of a limit-periodic potential by periodic ones), while in the present situation all considerations are happening in the space of the dual variable $\vec{\varkappa}$. The KAM methods in [30]-[32] and here are motivated by [33]-[35], where modifications of KAM method are used for periodic problems. Multiscale analysis which we apply here is essentially analogous to the original multiscale method developed in [36] (see also [37], 21]) for the proof of localization. The essential difference is that in [36], [37], [21] the multiscale procedure is constructed with respect to space variable $\vec{x}$ to prove localization, while we construct a multiscale procedure in the space of momenta $\vec{\varkappa}$ to prove delocalization.

Here is a brief description of the iteration procedure which leads to the results described above. Indeed, let $\vec{\varkappa} \in \mathbb{R}^{2}$. We consider a set of finite linear combinations of plane waves $e^{i\langle\vec{x}+2 \pi(\mathbf{n}+\alpha \mathbf{m}), \vec{x}\rangle}, \mathbf{n}, \mathbf{m} \in \mathbb{Z}^{2}$. The set is invariant under action of the differential expression 
(1). Let $H(\vec{\varkappa})$ be a matrix describing action of (11) in the linear set of the exponents. Obviously,

$$
\begin{gathered}
H(\overrightarrow{\mathcal{\varkappa}})=H_{0}(\vec{\varkappa})+V, \quad H_{0}(\overrightarrow{\mathcal{\varkappa}})_{(\mathbf{n}, \mathbf{m}),\left(\mathbf{n}^{\prime}, \mathbf{m}^{\prime}\right)}=|\overrightarrow{\mathcal{\varkappa}}+2 \pi(\mathbf{n}+\alpha \mathbf{m})|_{\mathbb{R}^{2}}^{2} \delta_{\left(\mathbf{n}, \mathbf{n}^{\prime}\right)} \delta_{\left(\mathbf{m}, \mathbf{m}^{\prime}\right)}, \\
V_{(\mathbf{n}, \mathbf{m}),\left(\mathbf{n}^{\prime}, \mathbf{m}^{\prime}\right)}=V_{\mathbf{n}-\mathbf{n}^{\prime}, \mathbf{m}-\mathbf{m}^{\prime} .}
\end{gathered}
$$

Next, we consider an expanding sequence of finite sets $\Omega_{n}$ in the space $\mathbb{Z}^{2} \times \mathbb{Z}^{2}$ of indices $(\mathbf{n}, \mathbf{m}): \Omega_{n} \subset \Omega_{n+1}, \lim _{n \rightarrow \infty} \Omega_{n}=\mathbb{Z}^{2} \times \mathbb{Z}^{2}$. Let $P_{n}$ be the characteristic projection of set $\Omega_{n}$ in the space $\ell^{2}\left(\mathbb{Z}^{2} \times \mathbb{Z}^{2}\right)$. We consider a sequence of finite matrices $H^{(n)}(\vec{\varkappa})=P_{n} H(\vec{\varkappa}) P_{n}$. Each matrix corresponds to a finite dimensional operator in $\ell^{2}\left(\mathbb{Z}^{2} \times \mathbb{Z}^{2}\right)$, given that the operator acts as zero on $\left(I-P_{n}\right) \ell^{2}$. For each $n$ we construct a "non-resonant" set $\mathcal{G}_{n}$ in the space $\mathbb{R}^{2}$ of momenta $\vec{\varkappa}$, such that: if $\vec{\varkappa} \in \mathcal{G}_{n}$, then $H^{(n)}(\vec{\varkappa})=P_{n} H(\vec{\varkappa}) P_{n}$ has an eigenvalue $\lambda_{n}(\vec{\varkappa})$ and its spectral projector $\mathcal{E}_{n}(\vec{\varkappa})$ which can be described by perturbation formulas with respect to the previous operator $H^{(n-1)}(\vec{\varkappa})$. If $\vec{\varkappa} \in \cap_{n=1}^{\infty} \mathcal{G}_{n}$ then $\lambda_{n}(\vec{\varkappa})$ and $\varepsilon_{n}(\vec{\varkappa})$ have limits. The linear combinations of the exponentials, corresponding to the projectors $\mathcal{E}_{n}(\vec{\varkappa})$, have a point-wise limit in $\vec{x}$, the limit being a generalized eigenfunction of (11). The generalized eigenfunction is close to the plane wave $e^{i\langle\vec{\varkappa}, \vec{x}\rangle}$ in the high energy region.

Each matrix $H^{(n)}$ is considered as a perturbation of a matrix $\hat{H}^{(n)}$, the latter has a block structure, i.e., consists of a variety of blocks $H^{(s)}(\vec{\varkappa}+2 \pi(\mathbf{n}+\alpha \mathbf{m})), s=1, \ldots, n-$ 1 , and, naturally, some diagonal terms. Blocks with different indices $(s)$ have sizes of different orders of magnitude (the size increasing with $s$ ). Thus we have a multiscale structure in the definition of $\hat{H}^{(n)}$. We use $\hat{H}^{(n)}(\vec{\varkappa})$ as a starting operator to construct perturbation series for $H^{(n)}(\vec{\varkappa})$. At a step $n$ we apply our knowledge of spectral properties of $H^{(s)}\left(\vec{\varkappa}+2 \pi\left(\mathbf{n}^{\prime}+\alpha \mathbf{m}^{\prime}\right)\right), s=1, \ldots, n-1, \mathbf{n}^{\prime}, \mathbf{m}^{\prime} \in \mathbb{Z}^{2}$, obtained in the previous steps, to describe spectral properties of $H^{(n)}(\vec{\varkappa}+2 \pi(\mathbf{n}+\alpha \mathbf{m})), \mathbf{n}, \mathbf{m} \in \mathbb{Z}^{2}$ and to construct $\mathcal{G}_{n}$.

At step one we use a regular perturbation theory and elementary geometric considerations to prove the following results. There is a set $\mathcal{G}_{1} \subset \mathbb{R}^{2}$ such that: if $\vec{\varkappa} \in \mathcal{G}_{1}$, then the operator $H^{(1)}(\vec{\varkappa})$ has a single eigenvalue close to the unperturbed one:

$$
\lambda^{(1)}(\vec{\varkappa}) \underset{|\vec{\varkappa}| \rightarrow \infty}{=}|\vec{\varkappa}|^{2}+O\left(|\vec{\varkappa}|^{-\gamma_{2}}\right), \quad \gamma_{2}>0 .
$$

A normalized eigenvector $\mathbf{u}^{(1)}$ is also close to the unperturbed one: $\mathbf{u}^{(1)}=\mathbf{u}^{(0)}+\tilde{\mathbf{u}}^{(1)}$, where $\left(\mathbf{u}^{(0)}\right)_{(\mathbf{n}, \mathbf{m})}=\delta_{\mathbf{n}, \mathbf{0}} \delta_{\mathbf{m}, \mathbf{0}}$ and the $l^{1}$-norm of $\tilde{\mathbf{u}}^{(1)}$ is small: $\left\|\tilde{\mathbf{u}}^{(1)}\right\|_{l^{1}}<|\vec{\imath}|^{-\gamma_{1}}, \gamma_{1}>0$. It follows that:

$$
\Psi_{1}(\overrightarrow{\mathcal{\varkappa}}, \vec{x})=e^{i\langle\vec{\varkappa}, \vec{x}\rangle}+\tilde{u}_{1}(\vec{\varkappa}, \vec{x}), \quad\left\|\tilde{u}_{1}\right\|_{L_{\infty}\left(\mathbb{R}^{2}\right)} \underset{|\vec{x}| \rightarrow \infty}{=} O\left(|\vec{\varkappa}|^{-\gamma_{1}}\right), \quad \gamma_{1}>0,
$$

where $\Psi_{1}(\vec{\varkappa}, \vec{x}), \tilde{u}_{1}(\vec{\varkappa}, \vec{x})$ are the linear combinations of the exponentials corresponding to vectors $\mathbf{u}^{(1)}$ and $\tilde{\mathbf{u}}^{(1)}$, respectively. It is shown that function $\Psi_{1}(\vec{\varkappa}, \vec{x})$ satisfies the equation for eigenfunctions with a good accuracy:

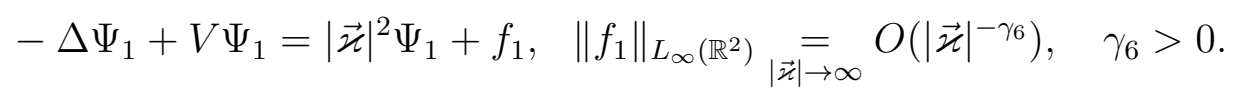


Relation (10) is differentiable:

$$
\nabla \lambda^{(1)}(\vec{\varkappa}) \underset{|\vec{\varkappa}| \rightarrow \infty}{=} 2 \vec{\varkappa}+O\left(|\vec{\varkappa}|^{-\gamma_{7}}\right), \quad \gamma_{7}>0
$$

Next, we construct a sequence $\mathcal{G}_{n}, n \geq 2$, such for any $\vec{\varkappa} \in \mathcal{G}_{n}$ the operator $H^{(n)}(\vec{\varkappa})$ has a single eigenvalue $\lambda^{(n)}(\vec{\varkappa})$ in a super exponentially small neighborhood of $\lambda^{(n-1)}(\vec{\varkappa})$ :

$$
\lambda^{(n)}(\vec{\varkappa}) \underset{|\vec{\varkappa}| \rightarrow \infty}{=} \lambda^{(n-1)}(\vec{\varkappa})+O\left(|\vec{\varkappa}|^{-|\vec{\varkappa}| \gamma^{\prime} n}\right), \quad \gamma_{8}>0 .
$$

Similar estimates hold for the eigenvectors and the corresponding functions $\Psi_{n}(\vec{\varkappa}, \vec{x})$ :

$$
\begin{array}{lll}
\Psi_{n}(\vec{\varkappa}, \vec{x})=\Psi_{n-1}(\vec{\varkappa}, \vec{x})+\tilde{u}_{n}(\vec{\varkappa}, \vec{x}), \quad\left\|\tilde{u}_{n}\right\|_{L_{\infty}\left(\mathbb{R}^{2}\right)} \underset{k \rightarrow \infty}{=} O\left(|\vec{\varkappa}|^{-|\vec{\varkappa}|^{\gamma_{9} n}}\right), & \gamma_{9}>0 . \\
-\Delta \Psi_{n}+V \Psi_{n}=\lambda^{(n)}(\vec{\varkappa}) \Psi_{n}+f_{n}, \quad\left\|f_{n}\right\|_{L_{\infty}\left(\mathbb{R}^{2}\right)} \underset{|\vec{\varkappa}| \rightarrow \infty}{=} O\left(|\vec{\varkappa}|^{-|\vec{\varkappa}|^{\gamma_{1} 0^{n}}}\right), & \gamma_{10}>0 .
\end{array}
$$

Formula (14) is differentiable with respect to $\vec{\varkappa}$ :

$$
\nabla \lambda^{(n)}(\vec{\varkappa}) \underset{|\vec{x}| \rightarrow \infty}{=} \nabla \lambda^{(n-1)}(\vec{\varkappa})+O\left(|\vec{\varkappa}|^{-|\vec{x}|^{\gamma_{8}{ }^{n}}}\right), \quad \gamma_{8}>0
$$

In fact, for large $n$ estimates (14) - (17) are even stronger. The non-resonant set $\mathcal{G}_{n}$ is proven to be extensive in $\mathbb{R}^{2}$ :

$$
\left|\mathcal{G}_{n} \cap \mathbf{B}_{\mathbf{R}}\right| \underset{R \rightarrow \infty}{=}\left|\mathbf{B}_{\mathbf{R}}\right|\left(1+O\left(R^{-\gamma_{3}}\right)\right) .
$$

Estimates (14) - (18) are uniform in $n$.

The set $\mathcal{D}_{n}(\lambda)$ is defined as the level (isoenergetic) set for the non-resonant eigenvalue $\lambda^{(n)}(\vec{\varkappa}):$

$$
\mathcal{D}_{n}(\lambda)=\left\{\vec{\varkappa} \in \mathcal{G}_{n}: \lambda^{(n)}(\vec{\varkappa})=\lambda\right\} .
$$

This set is proven to be a slightly distorted circle with a finite number of holes. The set $\mathcal{D}_{n}(\lambda)$ can be described by the formula:

$$
\mathcal{D}_{n}(\lambda)=\left\{\vec{\varkappa}: \vec{\varkappa}=\varkappa^{(n)}(\lambda, \vec{\nu}) \vec{\nu}, \vec{\nu} \in \mathcal{B}_{n}(\lambda)\right\},
$$

where $\mathcal{B}_{n}(\lambda)$ is a subset of the unit circle $S_{1}$. The set $\mathcal{B}_{n}(\lambda)$ can be interpreted as the set of possible directions of propagation for almost plane waves $\Psi_{n}(\vec{\varkappa}, \vec{x})$, see (11), (15). It has an asymptotically full measure on $S_{1}$ as $\lambda \rightarrow \infty$ :

$$
L\left(\mathcal{B}_{n}(\lambda)\right) \underset{\lambda \rightarrow \infty}{=} 2 \pi+O\left(\lambda^{-\gamma_{4}}\right) .
$$

Each set $\mathcal{B}_{n}(\lambda)$ has only a finite number of holes, however their number is growing with $n$. More and more holes of a smaller and smaller size are added at each step. The value 
$\varkappa^{(n)}(\lambda, \vec{\nu})-\lambda^{1 / 2}$ gives the deviation of $\mathcal{D}_{n}(\lambda)$ from the perfect circle of the radius $\lambda^{1 / 2}$ in the direction $\vec{\nu}$. It is proven that the deviation is asymptotically small:

$$
\varkappa^{(n)}(\lambda, \vec{\nu})=\lambda^{1 / 2}+O\left(\lambda^{-\gamma_{5}}\right), \quad \frac{\partial \varkappa^{(n)}(\lambda, \vec{\nu})}{\partial \varphi}=O\left(\lambda^{-\gamma_{11}}\right), \quad \gamma_{5}, \gamma_{11}>0,
$$

$\varphi$ being an angle variable, $\vec{\nu}=(\cos \varphi, \sin \varphi)$. Estimates (20), (21) are uniform in $n$.

On each step more and more points are excluded from the non-resonant sets $\mathcal{G}_{n}$, thus $\left\{\mathcal{G}_{n}\right\}_{n=1}^{\infty}$ is a decreasing sequence of sets. The set $\mathcal{G}_{\infty}$ is defined as the limit set: $\mathcal{G}_{\infty}=\cap_{n=1}^{\infty} \mathcal{G}_{n}$. It has an infinite number of holes, but nevertheless satisfies the relation (6). For every $\vec{\varkappa} \in \mathcal{G}_{\infty}$ and every $n$, there is a generalized eigenfunction of $H^{(n)}$ of the type (11), (15)). It is proven that the sequence of $\Psi_{n}(\vec{\varkappa}, \vec{x})$ has a limit in $L_{\infty}\left(\mathbb{R}^{2}\right)$ when $\vec{\varkappa} \in \mathcal{G}_{\infty}$. The function $\Psi_{\infty}(\vec{\varkappa}, \vec{x})=\lim _{n \rightarrow \infty} \Psi_{n}(\vec{\varkappa}, \vec{x})$ is a generalized eigenfunction of $H$. It can be written in the form (3i) - (44). Naturally, the corresponding eigenvalue $\lambda_{\infty}(\vec{\varkappa})$ is the limit of $\lambda^{(n)}(\vec{\varkappa})$ as $n \rightarrow \infty$.

It is shown that $\left\{\mathcal{B}_{n}(\lambda)\right\}_{n=1}^{\infty}$ is a decreasing sequence of sets, on each step more and more directions being excluded. We consider the limit $\mathcal{B}_{\infty}(\lambda)$ of $\mathcal{B}_{n}(\lambda)$ :

$$
\mathcal{B}_{\infty}(\lambda)=\bigcap_{n=1}^{\infty} \mathcal{B}_{n}(\lambda) .
$$

This set has a Cantor type structure on the unit circle. It is proven that $\mathcal{B}_{\infty}(\lambda)$ has an asymptotically full measure on the unit circle (see (8)). We prove that the sequence $\varkappa^{(n)}(\lambda, \vec{\nu}), n=1,2, \ldots$, describing the isoenergetic curves $\mathcal{D}_{n}(\lambda)$, quickly converges as $n \rightarrow \infty$. We show that $\mathcal{D}_{\infty}(\lambda)$ can be described as the limit of $\mathcal{D}_{n}(\lambda)$ in the sense (7), where $\varkappa_{\infty}(\lambda, \vec{\nu})=\lim _{n \rightarrow \infty} \varkappa^{(n)}(\lambda, \vec{\nu})$ for every $\vec{\nu} \in \mathcal{B}_{\infty}(\lambda)$. It is shown that the derivatives of the functions $\varkappa^{(n)}(\lambda, \vec{\nu})$ (with respect to the angle variable on the unit circle) have a limit as $n \rightarrow \infty$ for every $\vec{\nu} \in \mathcal{B}_{\infty}(\lambda)$. We denote this limit by $\frac{\partial \varkappa_{\infty}(\lambda, \vec{\nu})}{\partial \varphi}$. Using (21), we prove that

$$
\frac{\partial \varkappa_{\infty}(\lambda, \vec{\nu})}{\partial \varphi}=O\left(\lambda^{-\gamma_{11}}\right) .
$$

Thus, the limit curve $\mathcal{D}_{\infty}(\lambda)$ has a tangent vector in spite of its Cantor type structure, the tangent vector being the limit of corresponding tangent vectors for $\mathcal{D}_{n}(\lambda)$ as $n \rightarrow \infty$. The curve $\mathcal{D}_{\infty}(\lambda)$ looks as a slightly distorted circle with infinite number of holes for every sufficiently large $\lambda, \lambda>\lambda_{*}(V)$. It immediately follows that $\left[\lambda_{*}, \infty\right)$ is in the spectrum of $H$ (Bethe-Sommerfeld conjecture).

The main technical difficulty to overcome is the construction of non-resonant sets $\mathcal{B}_{n}(\lambda)$ for every fixed sufficiently large $\lambda, \lambda>\lambda_{0}(V)$, where $\lambda_{0}(V)$ is the same for all $n$. The set $\mathcal{B}_{n}(\lambda)$ is obtained by deleting a "resonant" part from $\mathcal{B}_{n-1}(\lambda)$. Definition of $\mathcal{B}_{n-1}(\lambda) \backslash$ $\mathcal{B}_{n}(\lambda)$ includes eigenvalues of $H^{(n-1)}(\vec{\varkappa})$. To describe $\mathcal{B}_{n-1}(\lambda) \backslash \mathcal{B}_{n}(\lambda)$ one has to consider not only non-resonant eigenvalues of the type (10), (14), but also resonant eigenvalues, for which no suitable formulas are known. Absence of formulas causes difficulties in 
estimating the size of $\mathcal{B}_{n-1}(\lambda) \backslash \mathcal{B}_{n}(\lambda)$. To treat this problem we start with introducing an angle variable $\varphi \in[0,2 \pi), \vec{\nu}=(\cos \varphi, \sin \varphi) \in S_{1}$ and consider sets $\mathcal{B}_{n}(\lambda)$ in terms of this variable. Next, we show that the resonant set $\mathcal{B}_{n-1}(\lambda) \backslash \mathcal{B}_{n}(\lambda)$ can be described as the set of zeros of functions of the type

$$
\operatorname{det}\left(H^{(s)}\left(\vec{\varkappa}_{n-1}(\varphi)+2 \pi(\mathbf{n}+\alpha \mathbf{m})\right)-\lambda-\varepsilon\right), \quad s=1, \ldots, n-1, \quad(\mathbf{n}, \mathbf{m}) \in \Omega_{n} \backslash(\mathbf{0}, \mathbf{0}),
$$

where $\vec{\varkappa}_{n-1}(\varphi)$ is a vector-function describing $\mathcal{D}_{n-1}(\lambda): \vec{\varkappa}_{n-1}(\varphi)=\varkappa_{n-1}(\lambda, \vec{\nu}) \vec{\nu}$. To obtain $\mathcal{B}_{n-1}(\lambda) \backslash \mathcal{B}_{n}(\lambda)$ we take all values of $\varepsilon$ in a small interval and $(\mathbf{n}, \mathbf{m})$ in some subset of $\Omega_{n}$. Further, we extend our considerations to a complex neighborhood $\Phi_{0}$ of $[0,2 \pi)$. We show that the determinants are analytic functions of $\varphi$ and, by this, reduce the problem of estimating the size of the resonant set to a problem in complex analysis. We use theorems for analytic functions to count zeros of the determinants and to investigate how far the zeros move when $\varepsilon$ changes. It enables us to estimate the size of the zero set of the determinants, and, hence, the size of the non-resonant set $\Phi_{n} \subset \Phi_{0}$, which is defined as a non-zero set for the determinants. Proving that the non-resonant set $\Phi_{n}$ is sufficiently large, we obtain estimates (18) for $\mathcal{G}_{n}$ and (20) for $\mathcal{B}_{n}$, the set $\mathcal{B}_{n}$ corresponding to the real part of $\Phi_{n}$. The essential part of constructing the nonresonant set is estimating the number of $(\mathbf{n}, \mathbf{m})$ in $\Omega_{n}$ for which (24) holds for a fixed real $\varphi$ and a sufficiently small values of $\varepsilon$. To obtain such an estimate we investigate geometric properties of the quasiperiodic lattice $\left\{\mathbf{n}+\alpha \mathbf{m},(\mathbf{n}, \mathbf{m}) \in \Omega_{n}\right\}$. Namely, we use algebraic and geometric considerations to obtain an estimate the number of lattice points inside a thin semi-agberaic set corresponding to zeros of (24) when $\varepsilon$ in a small neighborhood of zero.

To obtain $\Phi_{n}$ we delete from $\Phi_{0}$ more and more discs (holes) of smaller and smaller radii at each step. Thus, the non-resonant set $\Phi_{n} \subset \Phi_{0}$ has a structure of Swiss Cheese. Deleting a resonance set from $\Phi_{0}$ at each step of the recurrent procedure we call a "Swiss Cheese Method". The essential difference of our method from constructions of non-resonant sets in similar situations before (see e.g. [33]-[35], [38]) is that we construct a non-resonant set not only in the whole space of a parameter $\left(\vec{\varkappa} \in \mathbb{R}^{2}\right.$ here), but also on isoenergetic curves $\mathcal{D}_{n}(\lambda)$ in the space of the parameter, when $\lambda$ is sufficiently large. Estimates for the size of non-resonant sets on a curve require more subtle technical considerations than those sufficient for description of a non-resonant set in the whole space of the parameter. But as a reward, such estimates enable us to show that every isoenergetic set for $\lambda>\lambda_{0}$ is not empty and thus, to prove Bethe-Sommerfeld conjecture.

The plan of the paper is the following. Preliminary considerations are in Section 2. Sections $3-7$ describe steps of the recurrent procedure. Steps I,II are designed to start the procedure. Step I is quite simple and completely analogous to that in the case of the polyharmonic operator $(-\Delta)^{l}+V, l>1$, [1]. However, already the preparation for Step II (Section 3.5) is essentially more complicated in the present case of Schrödinger operator $(l=1)$ than in the case of the polyharmonic operator. It is caused by the fact, that $V(\vec{x})$ is a relatively stronger perturbation of $(-\Delta)^{l}$ at high energies in the case $l=1$ 
than in the case $l>1$. Mathematically, this means that the small denominator problem is more intricate for $l=1$. Even in the case of a periodic $V(\vec{x})$, Bloch eigenvalues of $(-\Delta)^{l}$ are located much denser at high energies in the case $l=1$ than in the case $l>1$. This means that description of their perturbation by a potential is a more challenging problem when $l=1$. In the case of a quasi-periodic potentials these difficulty, naturally, persists. The structure of the model operator for Step II is essentially more complicated here than that in [1]. In Step II we have to use the second condition on the potential (see the next section), which we did not impose in the case $l>1$. To construct the model block operator in Step II, we use one-dimensional periodic operators defined by "directional components" of $V(\vec{x})$ (by the second condition on the potential, they are periodic). Naturally, the proof of convergence of the perturbation series in Step II (Section 4.1) is more elaborated for $l=1$. Construction of the model operator in Step III requires understanding properties of a quasiperiodic lattice $\mathbf{n}+\alpha \mathbf{m}$ in a resonant set of $\vec{\varkappa}$. Resonant sets have more complicate structure here, than those in the case $l>1$. Therefore, the geometric part in the preparation for Step III (Section 4.3) is also trickier. Step III is already typical for the recurent procedure, however still uses some "non-typical" estimates from Steps I,II. This step is essentially the same for both $l>1$ and $l=1$ cases, except just a few places where results from Steps I,II are used. Step IV is completely typical: all other steps of the recurrent procedure differ from Step IV only by the change of indices. They are completely analogous for the cases $l=1$ and $l>1$. The proofs of convergence of the iteration procedure and of the results $1-3$, listed at the beginning of the introduction, are in Section 8. They are completely analogous to those in the polyharmonic case [1]. The result 4 on absolutely continuity of the spectrum is proven in Section 9 . The proof is also completely similar to that in [1]. Section 10 (Appendices) contains technical lemmas. For the sake of convenience we provide the list of the main notations at the end of the text (Section 11). In this text, for completeness of consideration, we provide all the proofs, even those which are analogous to the case $l>1$.

Acknowledgements. The authors are very grateful to Prof. Parnovski for useful discussions.

\section{Preliminary Remarks}

We consider two-dimensional quasi-periodic Schrödinger operator (11), which is perturbation of the free operator $H_{0}:=-\Delta$. Here the potential $V$ has the form (2), where $\alpha$ is an irrational number and $\mathcal{S}_{Q}=\mathcal{S}_{Q}(\alpha)$ is finite set. To simplify the construction we will assume that the set $\mathcal{S}_{Q}$ and $\alpha$ are not degenerate in some sense. More precisely, we impose the following conditions:

1) If $\mathbf{s}_{1}, \mathbf{s}_{2} \in \mathbb{Z}^{2}$ are such that $\mathbf{s}_{1}+\alpha \mathbf{s}_{2} \in \mathcal{S}_{Q}$ then $\left|\mathbf{s}_{1}\right|+\left|\mathbf{s}_{2}\right| \leq Q$.

2) If $\mathbf{s}_{1}, \mathbf{s}_{2}, \mathbf{s}_{1}^{\prime}, \mathbf{s}_{2}^{\prime} \in \mathbb{Z}^{2}$ are such that $\mathbf{s}_{1}+\alpha \mathbf{s}_{2}, \mathbf{s}_{1}^{\prime}+\alpha \mathbf{s}_{2}^{\prime} \in \mathcal{S}_{Q}$ and $\mathbf{s}_{1}+\alpha \mathbf{s}_{2}=c_{*}\left(\mathbf{s}_{1}^{\prime}+\alpha \mathbf{s}_{2}^{\prime}\right)$ 
then $c_{*}$ is rational. This means that if there are several vectors in $\mathcal{S}_{Q}$ with the same direction then they form a subset of a periodic one-dimensional lattice. Without loss of generality, we will assume that $\mathcal{S}_{Q}$ contains generating vectors $\mathbf{s}_{1}+\alpha \mathbf{s}_{2}$ of all present directions as well as all their integer multipliers $n\left(\mathbf{s}_{1}+\alpha \mathbf{s}_{2}\right), n \in \mathbb{Z}$, such that (see condition 1)) $|n|\left(\left|\mathbf{s}_{1}\right|+\left|\mathbf{s}_{2}\right|\right) \leq Q$. In particular, the set $\mathcal{S}_{Q}$ is symmetric with respect to $\mathbf{0}$ and $\mathbf{0} \in \mathcal{S}_{Q}$. We will assume though that $V_{\mathbf{0}, \mathbf{0}}=0$. As shown below in Lemma 2.1 the period of every one-dimensional sublattice in $\mathcal{S}_{Q}$ is not smaller than $C Q^{-\mu}$.

3) $\alpha: 0<\alpha<1$ is irrational and irrationality measure $\mu$ of $\alpha$ is finite: $\mu<\infty$ (in other words, this means that $\alpha$ is not a Liouville number). Note also, that $\mu \geq 2$ for any irrational number $\alpha$.

4) There are $N_{0}, N_{1}>0$ such that if $\left|n_{1}\right|+\left|n_{2}\right|+\left|n_{3}\right|>N_{1}$ then

$$
n_{1}+\alpha n_{2}+\alpha^{2} n_{3}=0 \quad \text { or } \quad\left|n_{1}+\alpha n_{2}+\alpha^{2} n_{3}\right|>\left(\left|n_{1}\right|+\left|n_{2}\right|+\left|n_{3}\right|\right)^{-N_{0}} .
$$

Note that the fourth condition is automatically satisfied for a quadratic irrational $\alpha$ :

$$
n_{1}^{\prime}+\alpha n_{2}^{\prime}+\alpha^{2} n_{3}^{\prime}=0
$$

for a triple $\left(n_{1}^{\prime}, n_{2}^{\prime}, n_{3}^{\prime}\right)$. Such triple is unique up to trivial multiplication (if exists), otherwise $\alpha$ is rational. If $n_{1}+\alpha n_{2}+\alpha^{2} n_{3} \neq 0$ for some other triple $\left(n_{1}, n_{2}, n_{3}\right)$, then (25) holds automatically, since otherwise $\mu=\infty$. The fourth condition is needed to estimate from below the angle between two non-colinear vectors $\mathbf{s}_{1}+\alpha \mathbf{s}_{2}$ and $\mathbf{s}_{1}^{\prime}+\alpha \mathbf{s}_{2}^{\prime}$ by a negative power of $\left|\mathbf{s}_{1}+\alpha \mathbf{s}_{2}\right|+\left|\mathbf{s}_{1}^{\prime}+\alpha \mathbf{s}_{2}^{\prime}\right|$.

It follows from the definition of the irrationality measure that 1) For any $\epsilon>0$ there exists a constant $C_{\varepsilon}$ such that for any irreducible rational number $\frac{\tilde{M}}{\tilde{N}}$ we have

$$
\left|\alpha-\frac{\tilde{M}}{\tilde{N}}\right| \geq \frac{C_{\varepsilon}}{\tilde{N}^{\mu+\epsilon}} .
$$

2) For any $\epsilon>0$ there exists a sequence $\frac{M}{N}$ of irreducible rational numbers such that

$$
\left|\alpha-\frac{M}{N}\right| \leq \frac{1}{N^{\mu-\epsilon}} .
$$

For simplicity we will often take $\epsilon=1$.

For every pair of integer vectors $\mathbf{s}_{1}, \mathbf{s}_{2} \in \mathbb{Z}^{2}$ we consider $\vec{p}_{\mathbf{s}}:=2 \pi\left(\mathbf{s}_{1}+\alpha \mathbf{s}_{2}\right)$. We introduce the norm

$$
||\left|\vec{p}_{\mathbf{s}}\right||:=| \mathbf{s}_{1}|+| \mathbf{s}_{2} \mid .
$$

We will also use the notation $p_{\mathbf{s}}:=\left|\vec{p}_{\mathbf{s}}\right|$ and $\vec{p}_{\mathbf{s}}=p_{\mathbf{s}}\left(\cos \varphi_{\mathbf{s}}, \sin \varphi_{\mathbf{s}}\right)$.

Lemma 2.1. For every $\vec{p}_{\mathbf{s}} \neq 0$ we have

$$
\begin{gathered}
p_{\mathbf{s}} \leq 2 \pi\left|\left\|\vec{p}_{\mathbf{s}}\right\|\right|, \\
p_{\mathbf{s}} \geq 2 \pi C_{\varepsilon}\left\|\left|\vec{p}_{\mathbf{s}} \|\right|^{-(\mu-1+\epsilon)} .\right.
\end{gathered}
$$


Proof. The estimate (29) is obvious. To prove (30) we notice that if $\mathbf{s}_{2}=0$ then $p_{\mathbf{s}}=2 \pi\left|\mathbf{s}_{1}\right| \geq 2 \pi$. Let $\mathbf{s}_{1}=\left(s_{11}, s_{12}\right), \mathbf{s}_{2}=\left(s_{21}, s_{22}\right)$. If, for example, $s_{21} \neq 0$ then from (27) and definition of $\left|\left\|\vec{p}_{\mathbf{s}}\right\|\right|$ we obtain

$$
\begin{aligned}
& p_{\mathbf{s}} \geq 2 \pi\left|s_{11}+\alpha s_{21}\right|=2 \pi\left|s_{21}\right|\left|\alpha+\frac{s_{11}}{s_{21}}\right| \geq \\
& 2 \pi C_{\varepsilon}\left|s_{21}\right|^{-\mu-\epsilon+1} \geq 2 \pi C_{\varepsilon}\left\|\left|\vec{p}_{\mathbf{s}} \|\right|^{-(\mu-1+\epsilon)} .\right.
\end{aligned}
$$

We introduce vector $\vec{\varkappa}(\varphi):=\left(\varkappa_{1}, \varkappa_{2}\right)=\varkappa \vec{\nu}:=\varkappa(\cos \varphi, \sin \varphi)$. Similar agreement will be used for other vectors. Let $H(\vec{\varkappa})=H(\varkappa, \varphi)$ be the "fiber" operator acting in $l^{2}\left(\mathbb{Z}^{4}\right)$ with its matrix elements given by

$$
(H(\vec{\varkappa}))_{\mathbf{s}, \mathbf{s}+\mathbf{q}}=\left|\vec{\varkappa}+\vec{p}_{\mathbf{s}}\right|^{2} \delta_{\mathbf{s}, \mathbf{s}+\mathbf{q}}+V_{\vec{p}_{\mathbf{q}}}
$$

Here $V_{\vec{p}_{\mathbf{q}}}:=V_{\mathbf{q}_{1}, \mathbf{q}_{2}}$ and $($ see (2) $)$

$$
V_{\vec{p}_{\mathbf{q}}}=0, \text { when }\left\|\mid \vec{p}_{\mathbf{q}}\right\| \|>Q, \quad(Q<\infty) .
$$

To simplify the notation in what follows we will write $V_{\mathbf{q}}$ instead of $V_{\vec{p}_{\mathbf{q}}}$ when it does not lead to confusion.

By $C, c$ we denote constants depending only on $V$, by $C_{0}, c_{0}$ we denote absolute constants.

\section{Step I}

\subsection{Operator $H^{(1)}$}

Let $\delta$ be some small parameter, $0<\delta<\left(10^{5} \mu\right)^{-1}$. We will also assume that $2 \delta N_{0} \leq 1 / 2$, $N_{0}$ being defined by (25). We put

$$
\Omega(\delta):=\left\{\mathbf{m} \in \mathbb{Z}^{4}: \quad|| \vec{p}_{\mathbf{m}} \mid \| \leq k^{\delta}\right\}, \quad \tilde{\Omega}(\delta):=\left\{\mathbf{m} \in \mathbb{Z}^{4}: \quad\left|\left\|\vec{p}_{\mathbf{m}} \mid\right\| \leq 4 k^{\delta}\right\} .\right.
$$

By $P(\delta)$ we denote an orthogonal (diagonal) projection in $l^{2}\left(\mathbb{Z}^{4}\right)$ on the set of elements supported in $\Omega(\delta)$. We call it the characteristic projector of $\Omega(\delta)$. The dimension of the projector is equal to the number of elements in $\Omega(\delta)$ and, obviously, does not exceed $\left(8 k^{\delta}\right)^{4}$. We have

$$
\left(P(\delta) H_{0}(\vec{\varkappa}) P(\delta)\right)_{\mathbf{m}, \mathbf{n}}=\left|\vec{\varkappa}+\vec{p}_{\mathbf{m}}\right|^{2} \delta_{\mathbf{m}, \mathbf{n}} \chi_{\Omega(\delta)}(\mathbf{m}),
$$

where as usual $\chi_{\Omega(\delta)}(\mathbf{m})$ is the characteristic function of the set $\Omega(\delta)$. We are going to consider $H^{(1)}(\vec{\varkappa})=P(\delta) H(\vec{\varkappa}) P(\delta)$ as a perturbation of the operator $P(\delta) H_{0}(\vec{\varkappa}) P(\delta)$. 


\subsection{Perturbation Formulas}

Now we construct a "non-resonant" set of $\varphi$, for which the operator $H^{(1)}(\vec{\varkappa}(\varphi))$ can be constructively considered as a perturbation of $H_{0}^{(1)}(\vec{\varkappa}(\varphi))$ corresponding to $V=0$. In what follows $\tau$ is an auxiliary parameter $\frac{1}{32} \leq \tau \leq 32$.

Lemma 3.1 (Geometric). For every $k>800$, there is a subset $\omega^{(1)}(k, \delta, \tau)$ of the interval $[0,2 \pi)$ such that:

1. For every $\varphi \in \omega^{(1)}(k, \delta, \tau)$ and $\mathbf{m} \in \tilde{\Omega}(\delta) \backslash\{0\}$, the following inequality holds:

$$
|| \vec{k}(\varphi)+\left.\vec{p}_{\mathbf{m}}\right|^{2}-k^{2} \mid>\tau k^{1-40 \mu \delta}, \quad \vec{k}:=k(\cos \varphi, \sin \varphi)
$$

2. For every $\varphi$ in the real $\frac{\tau}{16} k^{-(40 \mu+1) \delta}$-neighborhood of $\omega^{(1)}(k, \delta, \tau)$ and $\varkappa \in \mathbb{R}: \mid \varkappa-$ $k \mid<\frac{\tau}{16} k^{-40 \mu \delta}$, a slightly weaker inequality holds for $\vec{\varkappa}(\varphi)=\varkappa(\cos \varphi, \sin \varphi)$ and $\mathbf{m} \in \tilde{\Omega}(\delta) \backslash\{0\}:$

$$
|| \vec{\varkappa}(\varphi)+\left.\vec{p}_{\mathbf{m}}\right|^{2}-k^{2} \mid>\frac{\tau}{2} k^{1-40 \mu \delta}
$$

3. The set $\omega^{(1)}(k, \delta, \tau)$ has an asymptotically full measure in $[0,2 \pi)$ as $k \rightarrow \infty$. Namely,

$$
\left|\omega^{(1)}(k, \delta, \tau)\right|=2 \pi+O\left(k^{-37 \mu \delta}\right), \quad k \rightarrow \infty .
$$

Corollary 3.2. If $\varphi$ is in the real $\frac{\tau}{16} k^{-(40 \mu+1) \delta}$-neighborhood of $\omega^{(1)}(k, \delta, \tau)$ and $z$ is on the circle

$$
C_{1}=\left\{z:\left|z-k^{2}\right|=\frac{\tau}{4} k^{1-40 \mu \delta}\right\}
$$

then the following inequality holds for all $\mathbf{m} \in \tilde{\Omega}(\delta)$ :

$$
|| \vec{k}(\varphi)+\left.\vec{p}_{\mathbf{m}}\right|^{2}-z \mid \geq \frac{\tau}{4} k^{1-40 \mu \delta}, \quad z \in C_{1} .
$$

The lemma is proved in Section 3.3 (Corollaries 3.7 and 3.10, ) The corollary from the lemma is proven at the end of Section 3.3. Note that in Section 3.3 we construct non-resonance set of $\varphi$ in the set of complex numbers. Such complex non-resonance set we need for construction of further steps of approximation.

Let $r=1,2 \ldots$ and

$$
\begin{gathered}
g_{r}^{(1)}(\vec{\varkappa}):=\frac{(-1)^{r}}{2 \pi i r} \operatorname{Tr} \oint_{C_{1}}\left(\left(P(\delta)\left(H_{0}(\vec{\varkappa})-z I\right) P(\delta)\right)^{-1} V P(\delta)\right)^{r} d z \\
G_{r}^{(1)}(\vec{\varkappa}):=\frac{(-1)^{r+1}}{2 \pi i} \oint_{C_{1}}\left(\left(P(\delta)\left(H_{0}(\vec{\varkappa})-z I\right) P(\delta)\right)^{-1} V P(\delta)\right)^{r}\left(P(\delta)\left(H_{0}(\vec{\varkappa})-z I\right) P(\delta)\right)^{-1} d z .
\end{gathered}
$$


Note that $g_{1}^{(1)}(\vec{\varkappa})=0$ since $V=0$. Coefficient $g_{2}^{(1)}(\vec{\varkappa})$ admits representation:

$$
\begin{aligned}
g_{2}^{(1)}(\vec{\varkappa}) & =\sum_{\mathbf{q} \in \Omega(\delta) \backslash\{0\}}\left|V_{\mathbf{q}}\right|^{2}\left(|\vec{\varkappa}|^{2}-\left|\vec{\varkappa}+\vec{p}_{\mathbf{q}}\right|^{2}\right)^{-1} \\
& =-\sum_{\mathbf{q} \in \Omega(\delta) \backslash\{0\}} \frac{\left|V_{\mathbf{q}}\right|^{2}\left|\overrightarrow{p_{\mathbf{q}}}\right|^{2}}{\left(|\vec{\varkappa}|^{2}-\left|\vec{\varkappa}+\vec{p}_{\mathbf{q}}\right|^{2}\right)\left(|\vec{\varkappa}|^{2}-\left|\vec{\varkappa}-\vec{p}_{\mathbf{q}}\right|^{2}\right)},
\end{aligned}
$$

From now on $\|A\|_{1}$ means the norm of an operator $A$ in the trace class.

Theorem 3.3. Suppose $\varphi$ is in the real $\frac{\tau}{16} k^{-(40 \mu+1) \delta}$-neighborhood of $\omega^{(1)}(k, \delta, \tau)$ and $\varkappa \in$ $\mathbb{R},|\varkappa-k| \leq \frac{\tau}{16} k^{-40 \mu \delta}, \vec{\varkappa}=\varkappa(\cos \varphi, \sin \varphi)$. Then, for sufficiently large $k>k_{0}(V, \delta, \tau)$ there exists a single eigenvalue of $H^{(1)}(\vec{\varkappa})$ in the interval $\varepsilon_{1}(k, \delta, \tau)=\left(k^{2}-\frac{\tau}{2} k^{1-40 \mu \delta}, k^{2}+\right.$ $\left.\frac{\tau}{2} k^{1-40 \mu \delta}\right)$. It is given by the absolutely converging series:

$$
\lambda^{(1)}(\vec{\varkappa})=\varkappa^{2}+\sum_{r=2}^{\infty} g_{r}^{(1)}(\vec{\varkappa}) .
$$

For coefficients $g_{r}^{(1)}(\overrightarrow{\mathcal{\imath}})$ the following estimates hold:

$$
\left|g_{r}^{(1)}(\vec{\varkappa})\right| \leq(C k)^{-(r-1)(1-40 \mu \delta)+4 \delta}
$$

Moreover,

$$
\left|g_{2}^{(1)}(\vec{\varkappa})\right| \leq C k^{-2+(80 \mu+6) \delta} .
$$

The corresponding spectral projection is given by the series:

$$
\mathcal{E}^{(1)}(\vec{\varkappa})=\mathcal{E}_{0}(\vec{\varkappa})+\sum_{r=1}^{\infty} G_{r}^{(1)}(\vec{\varkappa}),
$$

$\mathcal{E}_{0}(\vec{\varkappa})$ being the unperturbed spectral projection. The operators $G_{r}^{(1)}(\vec{\varkappa})$ satisfy the estimates:

$$
\left\|G_{r}^{(1)}(\vec{\varkappa})\right\|_{1}<(C k)^{-r(1-44 \mu \delta)} .
$$

Matrix elements of $G_{r}^{(1)}(\vec{\varkappa})$ satisfy the following relations:

$$
G_{r}^{(1)}(\vec{\varkappa})_{\mathbf{s s}^{\prime}}=0, \quad \text { if } \quad r Q<\left\|\left|\vec { p } _ { \mathbf { s } } \left\|\left|+\left\|\left|\vec{p}_{\mathbf{s}^{\prime}} \|\right| .\right.\right.\right.\right.\right.
$$

Corollary 3.4. For the perturbed eigenvalue and its spectral projection the following estimates hold:

$$
\begin{aligned}
& \lambda^{(1)}(\vec{\varkappa})=\varkappa^{2}+O\left(k^{-2+(80 \mu+6) \delta}\right), \\
& \left\|\mathcal{E}^{(1)}(\vec{\varkappa})-\mathcal{E}_{0}(\vec{\varkappa})\right\|_{1}<c k^{-1+44 \mu \delta} .
\end{aligned}
$$

Matrix elements of spectral projection $\mathcal{E}^{(1)}(\vec{\varkappa})$ also satisfy the estimate:

$$
\left|\mathcal{E}^{(1)}(\vec{\varkappa})_{\mathbf{s s}^{\prime}}\right|<(C k)^{-d^{(1)}\left(\mathbf{s}, \mathbf{s}^{\prime}\right)}, \quad d^{(1)}\left(\mathbf{s}, \mathbf{s}^{\prime}\right)=Q^{-1}\left(\left\|\left|\vec { p } _ { \mathbf { s } } \left\|\left|+\left\|\left|\vec{p}_{\mathbf{s}^{\prime}} \|\right|\right)(1-44 \mu \delta) .\right.\right.\right.\right.\right.
$$


The last estimate easily follows from the formula (47) and estimate (46).

Proof. The proof is based on expansion of the resolvent in perturbation series on the circle $C_{1}$. Indeed, let us consider the series

$$
\left(H^{(1)}-z\right)^{-1}=\sum_{r=0}^{\infty}\left(H_{0}^{(1)}-z\right)^{-1}\left(-P(\delta) V P(\delta)\left(H_{0}^{(1)}-z\right)^{-1}\right)^{r}
$$

where $H_{0}^{(1)}=P(\delta) H_{0}$ and $z \in C_{1}$. It easily follows from (38) that

$$
\left\|\left(H_{0}^{(1)}(\vec{\varkappa})-z\right)^{-1}\right\|<\frac{8}{\tau} k^{-1+40 \mu \delta} .
$$

Hence,

$$
\left\|\left(H^{(1)}(\vec{\varkappa})-z\right)^{-1}\right\|<\frac{16}{\tau} k^{-1+40 \mu \delta}
$$

for sufficiently large $k$. Substituting the series into the formula $\mathcal{E}^{(1)}(\vec{\varkappa})=-\frac{1}{2 \pi i} \oint_{C_{1}}\left(H^{(1)}(\vec{\varkappa})-\right.$ $z)^{-1} d z$ and integrating term-wise, we arrive at (45). Estimates (46) easily follow from (52) and the obvious inequality $\|P(\delta)\|_{1} \leq\left(2 k^{\delta}\right)^{4}$. It follows $\mathcal{E}^{(1)}=\mathcal{E}_{0}+O\left(k^{-1+44 \mu \delta}\right)$. This means that there is a single eigenvalue of $H^{(1)}(\vec{\varkappa})$ inside $C_{1}$. In a similar way (using (39), (41) and $V=0$ ) we obtain the formula for the eigenvalue and (43), (44), for details see [39]. To prove (47) we consider the operator $A=V P(\delta)\left(H_{0}^{(1)}-z\right)^{-1}$ and represent it as $A=A_{0}+A_{1}+A_{2}$, where $A_{0}=\left(P(\delta)-\mathcal{E}_{0}(\vec{\varkappa})\right) A\left(P(\delta)-\mathcal{E}_{0}(\vec{\varkappa})\right)$, $A_{1}=\left(P(\delta)-\varepsilon_{0}(\vec{\varkappa})\right) A \varepsilon_{0}(\vec{\varkappa}), A_{2}=\varepsilon_{0}(\vec{\varkappa}) A\left(P(\delta)-\varepsilon_{0}(\vec{\varkappa})\right)$. It is easy to see that $\varepsilon_{0}(\vec{\varkappa}) A \varepsilon_{0}(\vec{\varkappa})=0$ because of $V=0$. Note that

$$
\oint_{C_{1}}\left(H_{0}^{(1)}-z\right)^{-1} A_{0}^{r} d z=0
$$

since the integrand is a holomorphic function inside $C_{1}$. Therefore,

$$
G_{r}^{(1)}(\vec{\varkappa})=\frac{(-1)^{r+1}}{2 \pi i} \sum_{j_{1}, \ldots j_{r}=0,1,2, j_{1}^{2}+\ldots+j_{r}^{2} \neq 0} \oint_{C_{1}}\left(H_{0}^{(1)}-z\right)^{-1} A_{j_{1}} \ldots . . A_{j_{r}} d z .
$$

At least one of indices in each term is equal to 1,2 . We take into account that $\left(A_{2}\right)_{\mathbf{s s}^{\prime}}=$ $\left(A_{1}\right)_{\mathbf{s}^{\prime} \mathbf{s}}=0$ if $\mathbf{s} \neq 0$ and $A_{\mathbf{s s}^{\prime}}=0$ if $\left\|\left|\vec{p}_{\mathbf{s}-\mathbf{s}^{\prime}} \|\right|>Q\right.$. It follows that $G_{r}^{(1)}(\vec{\varkappa})_{\mathbf{s s}^{\prime}}$ can differ from zero only if $r Q \geq\left\|\left|\vec{p}_{\mathbf{s}}\left\|\left|+\left\|\left|\vec{p}_{\mathbf{s}^{\prime}} \|\right|\right.\right.\right.\right.\right.$.

It will be shown (Corollary 3.8) that coefficients $g_{r}^{(1)}(\vec{\varkappa})$ and operators $G_{r}^{(1)}(\vec{\varkappa})$ can be analytically extended into the complex $\frac{\tau}{16} k^{-(40 \mu+1) \delta}$-neighborhood of $\omega^{(1)}(k, \delta, \tau)$ as functions of $\varphi$ and to the complex $\frac{\tau}{8} k^{-(40 \mu+1) \delta}$ - neighborhood of $k$ as functions of $\varkappa$, estimates (43), (44), (46) being preserved. Now, we use formulae (39), (42) to extend $\lambda^{(1)}(\vec{\varkappa})=\lambda^{(1)}(\varkappa, \varphi)$ as an analytic function. Obviously, series (42) is differentiable. Using Cauchy integral we get the following lemma. 
Lemma 3.5. Under conditions of Theorem 3.3 the following estimates hold when $\varphi$ is in $\omega^{(1)}(k, \delta, \tau)$ or its complex $\frac{\tau}{32} k^{-(40 \mu+1) \delta}$-neighborhood and $\varkappa$ is in the complex $\frac{\tau}{16} k^{-40 \mu \delta}$ neighborhood of $\varkappa=k$ :

$$
\begin{gathered}
\lambda^{(1)}(\vec{\varkappa})=\varkappa^{2}+O\left(k^{-2+(80 \mu+6) \delta}\right), \\
\frac{\partial \lambda^{(1)}}{\partial \varkappa}=2 \varkappa+O\left(k^{-2+(120 \mu+6) \delta}\right), \quad \frac{\partial \lambda^{(1)}}{\partial \varphi}=O\left(k^{-2+(120 \mu+7) \delta}\right), \\
\frac{\partial^{2} \lambda^{(1)}}{\partial \varkappa^{2}}=2+O\left(k^{-2+(160 \mu+6) \delta}\right), \\
\frac{\partial^{2} \lambda^{(1)}}{\partial \varkappa \partial \varphi}=O\left(k^{-2+(160 \mu+7) \delta}\right), \quad \frac{\partial^{2} \lambda^{(1)}}{\partial \varphi^{2}}=O\left(k^{-2+(160 \mu+8) \delta}\right) .
\end{gathered}
$$

\subsection{Geometric Considerations}

In this section we prove Lemma 3.1 and its corollary. However, we will prove a version of this lemma for a complex set of $\varphi$. We need this complex version for further steps. Lemma 3.1 is a simple corollary of the result proven in this section. We will use the notation $|\mathbf{a}|_{\mathbb{R}}^{2}:=(\mathbf{a}, \mathbf{a})_{\mathbb{R}}$ where $(\mathbf{a}, \mathbf{b})_{\mathbb{R}}:=a_{1} b_{1}+a_{2} b_{2}$ when $\mathbf{a}, \mathbf{b} \in \mathbb{C}^{2}$. It is easy to see that $\left|\vec{\varkappa}(\varphi)+\vec{p}_{\mathbf{m}}\right|_{\mathbb{R}}^{2}$ is an analytic extension in $\varkappa$ and $\varphi$ of

$$
\left|\vec{\varkappa}+\vec{p}_{\mathbf{m}}\right|^{2}=\varkappa^{2}+p_{\mathbf{m}}^{2}+2 \varkappa p_{\mathbf{m}} \cos \left(\varphi-\varphi_{\mathbf{m}}\right)
$$

defined for real $\varkappa, \varphi$. Note that $|\cdot|$ is the canonical norm in $\mathbb{C}$ or $\mathbb{R}^{2}$. For every fixed $k \geq 1$ and $\frac{1}{32} \leq \tau \leq 32$, we describe a resonance set $\mathcal{O}^{(1)}=\mathcal{O}^{(1)}(k, \tau)$ of $\varphi \in \mathbb{C}$. We put

$$
\mathcal{O}^{(1)}(k, \tau):=\cup_{\mathbf{m} \in \tilde{\Omega}(\delta) \backslash\{0\}} \mathcal{O}_{\mathbf{m}}(k, \tau),
$$

where

$$
\begin{aligned}
& \mathcal{O}_{\mathbf{m}}(k, \tau):=\left\{\varphi \in \mathbb{C}: \quad|| \vec{k}+\left.\vec{p}_{\mathbf{m}}\right|_{\mathbb{R}} ^{2}-k^{2} \mid \leq \tau k^{1-40 \mu \delta}\right\}= \\
& \left\{\varphi \in \mathbb{C}:\left|p_{\mathbf{m}}^{2}+2 k p_{\mathbf{m}} \cos \left(\varphi-\varphi_{\mathbf{m}}\right)\right| \leq \tau k^{1-40 \mu \delta}\right\} .
\end{aligned}
$$

In most cases parameter $\tau$ will be equal to 1 . But sometimes we will use different choice of $\tau$. It easily follows from the definition (58) and the estimate (29) that for any $\varkappa \in \mathbb{C}$ such that $|\varkappa-k| \leq 1$ and any $\varphi \in \mathcal{O}_{\mathbf{m}}(k, \tau)$ we have

$$
|| p_{\mathbf{m}}^{2}+2 \varkappa p_{\mathbf{m}} \cos \left(\varphi-\varphi_{\mathbf{m}}\right)|-| p_{\mathbf{m}}^{2}+2 k p_{\mathbf{m}} \cos \left(\varphi-\varphi_{\mathbf{m}}\right)|| \leq \frac{\tau}{4} k^{1-40 \mu \delta},
$$

provided $2(1+40 \mu) \delta \leq 1$ and $k \geq 800$ which will be assumed in what follows.

Let $\mathcal{W}_{0}:=\{\varphi \in \mathbb{C}:|\Im \varphi|<1\}$. We introduce a complex non-resonant set:

$$
\mathcal{W}^{(1)}(k, \tau):=\mathcal{W}_{0} \backslash \mathcal{O}^{(1)}(k, \tau) .
$$


Clearly, it is open. We also note that the set $\mathcal{O}^{(1)} \cap[0,2 \pi]$ is symmetric, i.e. $\mathcal{O}^{(1)} \cap[0,2 \pi]+$ $\pi(\bmod 2 \pi)=\mathcal{O}^{(1)} \cap[0,2 \pi]$, since $\varphi_{-\mathbf{m}}=\varphi_{\mathbf{m}}+\pi$. We define $\omega^{(1)}(k, \delta, \tau)$ as a real part of $\mathcal{W}^{(1)}(k, \delta, \tau)$ :

$$
\omega^{(1)}(k, \delta, \tau)=\mathcal{W}^{(1)}(k, \tau) \cap[0,2 \pi) .
$$

Lemma 3.6. Let $\varphi$ be in $\mathcal{W}^{(1)}(k, \tau)$, then

$$
|| \vec{k}(\varphi)+\left.\vec{p}_{\mathbf{m}}\right|_{\mathbb{R}} ^{2}-k^{2} \mid \geq \tau k^{1-40 \mu \delta} \text { for all } \mathbf{m} \in \tilde{\Omega}(\delta) \backslash\{0\} .
$$

If $\varphi$ is in the complex $k^{-(40 \mu+1) \delta}$-neighborhood of $\mathcal{W}^{(1)}(k, \tau)$ and $\varkappa \in \mathbb{C}:|\varkappa-k|<\frac{\tau}{8} k^{-40 \mu \delta}$. Then, for $\vec{\varkappa}=\varkappa(\cos \varphi, \sin \varphi)$ the following estimate holds:

$$
|| \vec{\varkappa}(\varphi)+\left.\vec{p}_{\mathbf{m}}\right|_{\mathbb{R}} ^{2}-k^{2} \mid \geq \frac{\tau}{2} k^{1-40 \mu \delta} \text { for all } \mathbf{m} \in \tilde{\Omega}(\delta) \backslash\{0\} .
$$

The lemma easily follows from (58) and (59).

Corollary 3.7. Parts 1 and 2 of Lemma 3.1 hold.

Corollary 3.8. Coefficients $g_{r}^{(1)}(\vec{\varkappa})$ and operators $G_{r}^{(1)}(\vec{\varkappa})$ can be analytically extended into the complex $\frac{\tau}{16} k^{-(40 \mu+1) \delta}$-neighborhood of $\omega^{(1)}(k, \delta, \tau)$ as functions of $\varphi$ and to the complex $\frac{\tau}{16} k^{-(40 \mu+1) \delta}$ - neighborhood of $k$ as functions of $\varkappa$, estimates (43), (44), (46) being preserved.

Lemma 3.9. The measure of the resonance set $\mathcal{O}^{(1)} \cap[0,2 \pi]$ satisfies the estimate:

$$
\operatorname{meas}\left(\mathcal{O}^{(1)} \cap[0,2 \pi]\right) \leq C_{0} k^{-37 \delta \mu} .
$$

Corollary 3.10. Part 3 of Lemma 3.1 holds.

Proof.

Let $\mathbf{m} \neq 0$ and $\varphi_{\mathbf{m}}^{ \pm}$be two $(\bmod 2 \pi)$ solutions of the equation

$$
p_{\mathbf{m}}^{2}+2 k p_{\mathbf{m}} \cos \left(\varphi-\varphi_{\mathbf{m}}\right)=0 .
$$

Obviously, $\varphi_{\mathbf{m}}^{ \pm}-\varphi_{\mathbf{m}}= \pm \frac{\pi}{2}+O\left(k^{-1+\delta}\right)$. Put

$$
\Phi_{\mathbf{m}}^{ \pm}:=\left\{\varphi \in \mathbb{C}:\left|\varphi-\varphi_{\mathbf{m}}^{ \pm}\right| \leq \tau k^{-39 \delta \mu}\right\} .
$$

Then, taking into account (30), it is not difficult to see that $\mathcal{O}_{\mathbf{m}} \subset \cup_{ \pm, j \in \mathbb{Z}}\left(\Phi_{\mathbf{m}}^{ \pm}+2 \pi j\right)$. Thus,

$$
\operatorname{meas}\left(\mathcal{O}^{(1)} \cap[0,2 \pi]\right) \leq 4 \tau k^{-39 \delta \mu}\left(8 k^{\delta}\right)^{4} \leq C k^{-37 \delta \mu} .
$$

Proof of Corollary 3.2, Let $C_{1}:=\left\{z \in \mathbb{C}:\left|z-k^{2}\right|=\frac{\tau}{4} k^{1-40 \mu \delta}\right\}$ be the contour around eigenvalue $k^{2}$ of the unperturbed operator $H_{0}(\vec{k})$. Then it follows from (62) that for any $\varphi \in \mathcal{W}^{(1)}(k, \tau), \mathbf{m} \in \tilde{\Omega}(\delta) \backslash\{0\}$, and $z:\left|z-k^{2}\right| \leq \frac{\tau}{4} k^{1-40 \mu \delta}$ we have

$$
\begin{aligned}
& || \vec{k}+\left.\vec{p}_{\mathbf{m}}\right|_{\mathbb{R}} ^{2}-z|\geq|\left|\vec{k}+\vec{p}_{\mathbf{m}}\right|_{\mathbb{R}}^{2}-k^{2} \mid-\frac{\tau}{4} k^{1-40 \mu \delta} \geq \\
& \tau k^{1-40 \mu \delta}-\frac{\tau}{4} k^{1-40 \mu \delta} \geq \frac{\tau}{4} k^{1-40 \mu \delta},
\end{aligned}
$$

for sufficiently large $k$. For $\mathbf{m}=0$ the estimate follows from the definition of $C_{1}$. 


\subsection{Isoenergetic Surface for Operator $H^{(1)}$}

Lemma 3.11. 1. For every sufficiently large $\lambda, \lambda:=k^{2}$, and $\varphi$ in the real $\frac{\tau}{32} k^{-(40 \mu+1) \delta}$ neighborhood of $\omega^{(1)}(k, \delta, \tau)$, there is a unique $\varkappa^{(1)}(\lambda, \varphi)$ in the interval $I_{1}:=[k-$ $\left.\frac{\tau}{32} k^{-40 \mu \delta}, k+\frac{\tau}{32} k^{-40 \mu \delta}\right]$, such that

$$
\lambda^{(1)}\left(\vec{\varkappa}^{(1)}(\lambda, \varphi)\right)=\lambda, \quad \vec{\varkappa}^{(1)}(\lambda, \varphi):=\varkappa^{(1)}(\lambda, \varphi) \vec{\nu}(\varphi) .
$$

2. Furthermore, there exists an analytic in $\varphi$ continuation of $\varkappa^{(1)}(\lambda, \varphi)$ to the complex $\frac{\tau}{32} k^{-(40 \mu+1) \delta}$-neighborhood of $\omega^{(1)}(k, \delta, \tau)$ such that $\lambda^{(1)}\left(\vec{\varkappa}^{(1)}(\lambda, \varphi)\right)=\lambda$. Function $\varkappa^{(1)}(\lambda, \varphi)$ can be represented as $\varkappa^{(1)}(\lambda, \varphi)=k+h^{(1)}(\lambda, \varphi)$, where

$$
\begin{gathered}
\left|h^{(1)}\right|=O\left(k^{-3+(80 \mu+6) \delta}\right), \\
\frac{\partial h^{(1)}}{\partial \varphi}=O\left(k^{-3+(120 \mu+7) \delta}\right), \quad \frac{\partial^{2} h^{(1)}}{\partial \varphi^{2}}=O\left(k^{-3+(160 \mu+8) \delta}\right), \\
\frac{\partial \varkappa^{(1)}}{\partial \lambda}=\frac{1}{2 k}\left(1+O\left(k^{-3+(120 \mu+6) \delta}\right)\right) .
\end{gathered}
$$

Proof.

1. Let us prove existence of $\varkappa^{(1)}(\lambda, \varphi)$. By Theorem 3.3, there exists an eigenvalue $\lambda^{(1)}(\vec{\varkappa})$, given by (42), for all $\varkappa$ in the interval $I_{1}$. Let $\mathcal{L}^{(1)}(\varphi):=\left\{\lambda^{(1)}(\vec{\varkappa}): \varkappa \in I_{1}\right\}$. Using the definition of $I_{1}$, (48), and continuity of $\lambda^{(1)}(\vec{\varkappa})$ in $\varkappa$, we easily obtain $\mathcal{L}^{(1)}(\varphi) \supset\left[k^{2}-t, k^{2}+t\right], t=c_{1} k^{1-40 \mu \delta}, 0<c_{1} \neq c_{1}(k)$. Hence, there exists a $\varkappa^{(1)}$ such that $\lambda^{(1)}\left(\vec{\varkappa}^{(1)}\right)=k^{2}, \varkappa^{(1)} \in I_{1}$.

Now we show that there is only one $\varkappa^{(1)}$ in the interval $I_{1}$ satisfying (67). Indeed, by $(\underline{55}), \frac{\partial \lambda^{(1)}(\vec{\varkappa})}{\partial \varkappa} \geq 2 k(1+o(1))$. This implies that $\lambda^{(1)}(\vec{\varkappa})$ is monotone with respect to $\varkappa$ in $I_{1}$. Thus, there is only one $\varkappa \in I_{1}$ satisfying (67).

2. We consider $\lambda^{(1)}(\vec{\varkappa}(\varphi))$ as a function of complex variable $\varkappa$ in the disc $|\varkappa-k|<$ $\frac{\tau}{32} k^{-40 \mu \delta}$. Taking into account (54) and applying Rouché's Theorem, we obtain that for any $\varphi$ in $\frac{\tau}{32} k^{-(40 \mu+1) \delta}$-neighborhood of $\omega^{(1)}(k, \delta, \tau)$ there exists unique value of $\varkappa^{(1)}(\varphi)$ such that $\left|\varkappa^{(1)}(\varphi)-k\right|<\frac{\tau}{32} k^{-40 \mu \delta}$ and $\lambda^{(1)}\left(\vec{\varkappa}^{(1)}(\varphi)\right)=\lambda:=k^{2}$. Actually (see $(\underline{54})$ ),

$$
\left|\varkappa^{(1)}(\varphi)-k\right|=O\left(k^{-3+(80 \mu+6) \delta}\right) .
$$

Then it follows from (55) and implicit function theorem that $\varkappa^{(1)}(\varphi)$ is locally analytic. Combined with uniqueness this implies global analyticity.

The estimate (68) follows from (71). Applying standard arguments with the Cauchy formula we obtain (69). Using (55) we get (70). 
Let us consider the set of points in $\mathbb{R}^{2}$ given by the formula: $\vec{\varkappa}=\vec{\varkappa}^{(1)}(\varphi), \quad \varphi \in$ $\omega^{(1)}(k, \delta, \tau)$. By Lemma 3.11 this set of points is a slightly disturbed circle with holes. All the points of this curve satisfy the equation $\lambda^{(1)}\left(\vec{\varkappa}^{(1)}(\lambda, \varphi)\right)=k^{2}$. We call it isoenergetic surface of the operator $H^{(1)}$ and denote by $\mathcal{D}_{1}(\lambda)$. The "radius" $\varkappa^{(1)}(\lambda, \varphi)$ of $\mathcal{D}_{1}(\lambda)$ monotonously increases with $\lambda$, see (170).

\subsection{Preparation for Step II. Construction of the Second Non- resonant Set}

\subsubsection{Model Operator for Step II}

Here we will describe an operator $P H P$, see (150), which will be used for constructing perturbation series in the second step. The operator $P H P$ has a block structure.

Let $r_{1}$ be some fixed number $2<r_{1}$. An upper bound on $r_{1}$ we will introduce in Step II. We defined $\mathcal{O}_{\mathbf{m}}$ by formula (58) for all $\mathbf{m}: 0<\left\|\left|\vec{p}_{\mathbf{m}} \|\right| \leq 4 k^{\delta}\right.$. Now, we define $\mathcal{O}_{\mathbf{m}}$ for $\mathbf{m}: 4 k^{\delta}<\left\|\left|\vec{p}_{\mathbf{m}} \|\right| \leq k^{r_{1}}\right.$ by the following formula:

$$
\begin{aligned}
& \mathcal{O}_{\mathbf{m}}(k, \tau):=\left\{\varphi \in \mathbb{C}:|| \vec{k}+\left.\vec{p}_{\mathbf{m}}\right|_{\mathbb{R}} ^{2}-k^{2} \mid \leq \tau k^{\delta_{*}}\right\}= \\
& \left\{\varphi \in \mathbb{C}:\left|p_{\mathbf{m}}^{2}+2 k p_{\mathbf{m}} \cos \left(\varphi-\varphi_{\mathbf{m}}\right)\right| \leq \tau k^{\delta_{*}}\right\}, \quad \delta_{*}=10^{4} \mu \delta<1 / 10 .
\end{aligned}
$$

Note that the right-hand part in the inequality here is smaller than the corresponding one in (58). Obviously, $\mathcal{O}_{\mathbf{m}}$ contains the whole interval $[0,2 \pi)$ for sufficiently small $p_{\mathbf{m}}$. As in Step I let $\varphi_{\mathbf{m}}^{ \pm}$be two $(\bmod 2 \pi)$ solutions of the equation

$$
p_{\mathbf{m}}^{2}+2 k p_{\mathbf{m}} \cos \left(\varphi-\varphi_{\mathbf{m}}\right)=0 .
$$

Lemma 3.12. The set $\mathcal{O}_{\mathbf{m}}(k, \tau)$ has the following properties:

1. If $p_{\mathbf{m}}>4 k$, then $\mathcal{W}_{0} \cap \mathcal{O}_{\mathbf{m}}(k, \tau)=\emptyset$.

2. If $k^{-1+2 \delta_{*}} \leq p_{\mathbf{m}} \leq 4 k$ and $\left|4 k^{2}-p_{\mathbf{m}}^{2}\right|>4 \tau k^{\delta_{*}}$, then $\mathcal{O}_{\mathbf{m}} \subset \cup_{ \pm, j \in \mathbb{Z}}\left(\Phi_{\mathbf{m}}^{ \pm}+2 \pi j\right)$, where

$$
\Phi_{\mathbf{m}}^{ \pm}:=\left\{\varphi \in \mathbb{C}: \quad\left|\varphi-\varphi_{\mathbf{m}}^{ \pm}\right| \leq \frac{\tau k^{-1+\delta_{*}}}{p_{\mathbf{m}} \sqrt{1-p_{\mathbf{m}}^{2}(2 k)^{-2}}}\right\},
$$

and $\Phi_{\mathbf{m}}^{+} \cap \Phi_{\mathbf{m}}^{-}=\emptyset$.

3. If $\left|4 k^{2}-p_{\mathbf{m}}^{2}\right| \leq 4 \tau k^{\delta_{*}}$, then $\mathcal{O}_{\mathbf{m}} \subset \cup_{ \pm, j \in \mathbb{Z}}\left(\Phi_{\mathbf{m}}^{ \pm}+2 \pi j\right)$, where

$$
\Phi_{\mathbf{m}}^{ \pm}:=\left\{\varphi \in \mathbb{C}:\left|\varphi-\varphi_{\mathbf{m}}^{ \pm}\right| \leq 32 \tau k^{-1+\delta_{*} / 2}\right\}
$$


In the proof we use the Taylor series with respect to $\varphi$ for $\left|\vec{k}(\varphi)+\vec{p}_{\mathbf{m}}\right|_{\mathbb{R}}^{2}-k^{2}$ near its zeros, see Appendix 1.

Let $\varphi_{0} \in[0,2 \pi) \backslash \mathcal{O}^{(1)}(k, 8)$, where $\mathcal{O}^{(1)}(k, 8)$ is given by (57). We define $\mathcal{M}\left(\varphi_{0}\right) \subset \mathbb{Z}^{4}$ as follows:

$$
\mathcal{M}\left(\varphi_{0}\right):=\left\{\mathbf{m}: \quad 0<||\left|\vec{p}_{\mathbf{m}}\right| \| \leq k^{r_{1}} \text { and } \varphi_{0} \in \mathcal{O}_{\mathbf{m}}(k, 1)\right\} .
$$

We will also need a larger set

$$
\mathcal{M}^{\prime}\left(\varphi_{0}\right):=\left\{\mathbf{m}: \quad 0<\left|\left\|\vec{p}_{\mathbf{m}} \mid\right\| \leq 2 k^{r_{1}} \text { and } \varphi_{0} \in \mathcal{O}_{\mathbf{m}}(k, 1)\right\} .\right.
$$

In fact, $\mathcal{M}\left(\varphi_{0}\right), \mathcal{M}^{\prime}\left(\varphi_{0}\right)$ do not include $\mathbf{m}:\left|\left\|\vec{p}_{\mathbf{m}} \mid\right\|<4 k^{\delta}\right.$, since $\varphi_{0} \in[0,2 \pi) \backslash \mathcal{O}^{(1)}(k, 8)$.

We split $\mathcal{M}\left(\varphi_{0}\right)$ into two components $\mathcal{M}:=\mathcal{M}_{1} \cup \mathcal{M}_{2}$. By definition, $\mathbf{m} \in \mathcal{M}_{1}$ if

$$
\min _{\mathbf{m}^{\prime} \in \mathcal{M}^{\prime}(\varphi), \mathbf{m}^{\prime} \neq \mathbf{m}}\left\|\left|\vec{p}_{\mathbf{m}-\mathbf{m}^{\prime}} \|\right|>k^{\delta} .\right.
$$

Let $\mathcal{M}_{2}=\mathcal{M} \backslash \mathcal{M}_{1}$. Next, let $\tilde{\mathcal{M}}_{\mathbf{m}}$ be $\left(k^{\delta} / 3\right)$-neighborhood of $\mathbf{m} \in \mathcal{M}$ in $\||\cdot \||$ norm:

$$
\tilde{\mathcal{M}}_{\mathbf{m}}:=\left\{\mathbf{n}: \quad\left|\left\|\vec{p}_{\mathbf{n}-\mathbf{m}} \mid\right\|<k^{\delta} / 3 \text { for a given } \mathbf{m} \in \mathcal{M}\left(\varphi_{0}\right)\right\} .\right.
$$

Obviously,

$$
\tilde{\mathcal{M}}_{\mathbf{m}}\left(\varphi_{0}\right) \cap \tilde{\mathcal{M}}_{\mathbf{m}^{\prime}}\left(\varphi_{0}\right)=\emptyset, \quad \text { for any } \mathbf{m} \in \mathcal{M}_{1} \text { and } \mathbf{m}^{\prime} \in \mathcal{M}^{\prime}, \quad \mathbf{m}^{\prime} \neq \mathbf{m} .
$$

Let $\tilde{\mathcal{M}}_{1}\left(\varphi_{0}\right)$ be $\left(k^{\delta} / 3\right)$-neighborhood of $\mathcal{M}_{1}$ in $\||\cdot \||$ norm:

$$
\tilde{\mathcal{M}}_{1}\left(\varphi_{0}\right):=\cup_{\mathbf{m} \in \mathcal{M}_{1}\left(\varphi_{0}\right)} \tilde{\mathcal{M}}_{\mathbf{m}}\left(\varphi_{0}\right)=\left\{\mathbf{n}: \quad|| \vec{p}_{\mathbf{n}-\mathbf{m}} \mid \|<k^{\delta} / 3 \text { for some } \mathbf{m} \in \mathcal{M}_{1}\left(\varphi_{0}\right)\right\} .
$$

Let us introduce an equivalence relation in $\mathcal{M}^{\prime}$. We say $\mathbf{m}_{0} \sim \mathbf{m}_{0}^{\prime}$ if there is a sequence $\mathbf{m}_{j} \in \mathcal{M}^{\prime}, j=1, \ldots, J$, connecting these two points $\left(\mathbf{m}_{J}=\mathbf{m}_{0}^{\prime}\right)$, such that for each $j$ $\min _{k: 0 \leq k<j}\left\|\left|\vec{p}_{\mathbf{m}_{j}-\mathbf{m}_{k}} \|\right| \leq 3 k^{\delta}\right.$. We denote the equivalence class containing $\mathbf{m} \in \mathcal{M}_{2}$ by $\mathcal{M}_{2}^{(\mathbf{m})}$. By definition of $\mathcal{M}_{2}$ such equivalence class contains at least one more element.

Lemma 3.13. Let $\mathbf{m}_{0} \in \mathcal{M}_{2}$ and $\mathbf{m}_{j} \in \mathcal{M}^{\prime}, j=1,2$, are such that all $\mathbf{m}_{j}, j=0,1,2$, are different and $\min _{k: k \neq j}\left\|\left|\vec{p}_{\mathbf{m}_{j}-\mathbf{m}_{k}} \|\right| \leq 3 k^{\delta}\right.$ for all $j=0,1,2$. Then, $\vec{p}_{\mathbf{m}_{1}-\mathbf{m}_{0}}=c \vec{p}_{\mathbf{m}_{2}-\mathbf{m}_{0}}$ with some $c \in \mathbb{R}$.

Corollary 3.14. All points $\vec{p}_{\mathbf{m}^{\prime}}$ with $\mathbf{m}^{\prime}$ being in a class of equivalence $\mathcal{M}_{2}^{(\mathbf{m})}$ are situated on a line.

The proof of the lemma is in Appendix 2.

Obviously, for any pair $\mathbf{m}, \mathbf{m}^{\prime} \in \mathcal{M}_{2}$ either $\mathcal{M}_{2}^{(\mathbf{m})}=\mathcal{M}_{2}^{\left(\mathbf{m}^{\prime}\right)}$ or $\mathcal{M}_{2}^{(\mathbf{m})} \cap \mathcal{M}_{2}^{\left(\mathbf{m}^{\prime}\right)}=\emptyset$. We can enumerate different equivalence classes $\mathcal{M}_{2}^{(\mathbf{m})}$ by an index $j$ and denote them by 
$\mathcal{M}_{2}^{j}, j=1, \ldots, J_{0}$. By construction, $\mathcal{M}_{2} \subset \cup_{j=1}^{J_{0}} \mathcal{M}_{2}^{j} \subset \mathcal{M}^{\prime}$. By Lemma 3.13 each class $\mathcal{M}_{2}^{j}$ has a "direction", which is the direction of the corresponding line. We denote it by $\vec{p}_{\mathbf{q}}, \vec{p}_{\mathbf{q}}=\vec{p}_{\mathbf{m}_{1}-\mathbf{m}_{0}}$, the direction, naturally, being defined up to a constant multiplier. Obviously, all vectors $\vec{p}_{\mathbf{m}}, \mathbf{m} \in \mathcal{M}_{2}^{j}$, have the same projection on a direction $\vec{\nu}_{\mathbf{q}}^{\perp}$ orthogonal to $\vec{\nu}_{\mathbf{q}}:=\vec{p}_{\mathbf{q}} / p_{\mathbf{q}}$. We define the "orthogonal component" $t_{\mathbf{q}}^{\perp}$ of $\mathcal{M}_{2}^{j}$ as

$$
t_{\mathbf{q}}^{\perp}=\left(\vec{k}\left(\varphi_{0}\right)+\vec{p}_{\mathbf{m}}, \vec{\nu}_{\mathbf{q}}^{\perp}\right), \quad \mathbf{m} \in \mathcal{M}_{2}^{j}
$$

Definition 3.15. We call $\mathcal{M}_{2}^{j}$ trivial if one of two conditions holds:

1) no vector in $\mathcal{S}_{Q}$ has the same direction as $\mathcal{M}_{2}^{j}$ or

2) there is $\mathbf{q} \in \mathcal{S}_{Q}$ which has the same direction as $\mathcal{M}_{2}^{j}$ and $t_{\mathbf{q}}^{\perp}$ satisfies the inequality:

$$
\left|k^{2}-\left(t_{\mathbf{q}}^{\perp}\right)^{2}\right|>\frac{1}{8} k^{\delta_{*}}
$$

Otherwise, we call a cluster non-trivial. In a non-trivial case, without the loss of generality, we can consider that the directional vector $\mathbf{q}$ of $\mathcal{M}_{2}^{j}$ belongs to $\mathcal{S}_{Q}$ and it is a generating vector in its direction (see the definition of $\mathcal{S}_{Q}$ ).

When a cluster is trivial, it can be treated by a method quite similar to that in the case of a polyharmonic operator. For non-trivial clusters we will use an additional consideration, involving a periodic operator in one dimension. It is a periodic and not a quasiperiodic operator, because of condition 2 on $V(\vec{x})$ at the beginning of Section 2 . Treatment of non-trivial clusters is the main reason we need condition 2 . We did not need condition 2 on $V(\vec{x})$ for the polyharmonic case [1], since in that case all clusters $\mathcal{M}_{2}^{j}$ could be treated the same way as trivial ones here.

Now we introduce a further split of every $\mathcal{M}_{2}^{j}$. Suppose $\mathcal{M}_{2}^{j}$ is non-trivial. For $\mathbf{m}, \mathbf{m}^{\prime} \in$ $\mathcal{M}_{2}^{j}$ we say $\mathbf{m} \sim \mathbf{m}^{\prime}$ if $\mathbf{m}-\mathbf{m}^{\prime}=n \mathbf{q}, n \in \mathbb{Z}, \mathbf{q}$ is the directional vector of $\mathcal{M}_{2}^{j}$ in $\mathcal{S}_{Q}$ as described above. Hence, every $\mathcal{M}_{2}^{j}$ is represented as a disjoint union of such subsets, $\mathcal{M}_{2}^{j}=\cup_{s} \mathcal{M}_{2}^{j, s}$. We notice that every set $\mathcal{M}_{2}^{j, s}$ consists of vectors of the form $\mathbf{m}_{j, s}+n \mathbf{q}$, where $n \in \mathbb{Z}$ and such that $\mathbf{m}_{j, s}+n \mathbf{q} \in \mathcal{M}_{2}^{j}, \mathbf{q}=\mathbf{q}(j)$. By (174), (172),

$$
|| \vec{k}\left(\varphi_{0}\right)+\vec{p}_{\mathbf{m}_{j, s}}+\left.n \vec{p}_{\mathbf{q}}\right|^{2}-k^{2} \mid<k^{\delta_{*}} .
$$

Considering the inequality opposite to (77), we obtain that each $\mathcal{M}_{2}^{j, s}$ can be described by the formula:

$$
\mathcal{M}_{2}^{j, s}=\left\{\mathbf{m}_{j, s}+n \mathbf{q}: 0 \leq\left(\vec{k}\left(\varphi_{0}\right)+\vec{p}_{\mathbf{m}_{j, s}}, \vec{\nu}_{\mathbf{q}}\right)<p_{\mathbf{q}}, n \in \mathbb{Z}, n_{-}^{j, s} \leq n \leq n_{+}^{j, s}\right\},
$$

where $n_{-}^{j, s}<0, n_{+}^{j, s}>0, \frac{1}{2} p_{\mathbf{q}}^{-1} k^{\delta_{*} / 2}<\left|n_{-}^{j, s}\right|, n_{+}^{j, s}<2 p_{\mathbf{q}}^{-1} k^{\delta_{*} / 2}$ and

$$
|| \vec{k}\left(\varphi_{0}\right)+\vec{p}_{\mathbf{m}_{j, s}}+\left.n_{ \pm}^{j, s} \vec{p}_{\mathbf{q}}\right|^{2}-k^{2} \mid=k^{\delta_{*}}+O\left(k^{\delta_{*} / 2}\right) .
$$


It is easy to see also that

$$
\left|n_{ \pm}^{j, s}-n_{ \pm}^{j, s^{\prime}}\right| \leq 1
$$

for any pair $s, s^{\prime}$. We will refer the point $\mathbf{m}_{j, s}$ as the central point of $\mathcal{M}_{2}^{j, s}$. We also have $\left|\vec{p}_{\mathbf{q}}\right| \geq C Q^{-\mu}$.

In the case when $\mathcal{M}_{2}^{j}$ is trivial, we consider that each $\mathcal{M}_{2}^{j, s}$ contains just one point.

In a non-trivial case the reduction $H^{j, s}\left(\vec{k}\left(\varphi_{0}\right)\right):=P_{j, s} H\left(\vec{k}\left(\varphi_{0}\right)\right) P_{j, s}$ (here $P_{j, s}$ is the diagonal projection in $l^{2}$ corresponding to $\left.\mathcal{M}_{2}^{j, s}\right)$ can be described by the matrix:

$$
H_{n n^{\prime}}^{j, s}=\left|\vec{k}\left(\varphi_{0}\right)+\vec{p}_{\mathbf{m}_{j, s}}+n \vec{p}_{\mathbf{q}(j)}\right|_{\mathbb{R}}^{2} \delta_{n, n^{\prime}}+V_{\left(n-n^{\prime}\right) \mathbf{q}(j)} .
$$

with $\mathbf{m}_{j, s}+n \mathbf{q}, \mathbf{m}_{j, s}+n^{\prime} \mathbf{q} \in \mathcal{M}_{2}^{j, s}\left(n_{-}^{j, s} \leq n, n^{\prime} \leq n_{+}^{j, s}\right)$. Note that there is a "separation of variables", i.e., this matrix can be rewritten as

$$
H^{j, s}=\tilde{H}^{j, s}\left(t_{\mathbf{q}}\right)+\left(t_{\mathbf{q}}^{\perp}\right)^{2} I,
$$

where

$$
\begin{gathered}
\tilde{H}_{n_{1}, n_{2}}^{j, s}:=\left(t_{\mathbf{q}}+n_{1} p_{\mathbf{q}}\right)^{2} \delta_{n_{1}, n_{2}}+V_{\left(n_{1}-n_{2}\right) \mathbf{q}} \\
t_{\mathbf{q}}:=\left(\vec{k}\left(\varphi_{0}\right)+\vec{p}_{\mathbf{m}_{j, s}}, \vec{\nu}_{\mathbf{q}}\right), \quad t_{\mathbf{q}}^{\perp}:=\left(\vec{k}\left(\varphi_{0}\right)+\vec{p}_{\mathbf{m}_{j, s}}, \vec{\nu}_{\mathbf{q}}^{\perp}\right) .
\end{gathered}
$$

Note that $t_{\mathbf{q}}$ depends both on $j$ and $s$, while $t_{\mathbf{q}}^{\perp}$ depends on $j$ only (we omit indices $j, s$ for shortness). By construction, $\mathbf{q}=\mathbf{q}(j) \in \mathcal{S}_{Q}$ and $0 \leq t_{\mathbf{q}}<p_{\mathbf{q}}$. Let us consider also an infinite version of (83) $\tilde{H}_{\text {per }}^{j, s}$, i.e. $\left(\tilde{H}_{p e r}^{j, s}\right)_{n n^{\prime}}$ is given by (83) for all $n, n^{\prime} \in \mathbb{Z}$. Clearly, $\tilde{H}_{\text {per }}^{j, s}$ corresponds to a one-dimensional Schroedinger operator with a periodic potential and quasimomentum $t_{\mathbf{q}}$. Obviously, this construction works not only for $\varphi_{0}$, but also for any $\varphi$ in the $2 k^{-2-40 \mu \delta}$-neighborhood of $\varphi_{0}$. We are going to investigate properties of $H^{j, s}$ using known properties of $\tilde{H}_{p e r}^{j, s}$.

Lemma 3.16. Let $\left|\Re \varphi-\varphi_{0}\right| \leq 2 k^{-2-40 \mu \delta}$. Then $\Im\left|\vec{k}(\varphi)+\vec{p}_{\mathbf{n}}\right|_{\mathbb{R}}^{2}$ have the same sign for all $\mathbf{n} \in \mathcal{M}_{2}^{j, s}$ when $\Im \varphi>0$ (or $\Im \varphi<0$ ) and the following inequality holds:

$$
|\Im| \vec{k}(\varphi)+\left.\vec{p}_{\mathbf{n}}\right|_{\mathbb{R}} ^{2}\left|\geq k^{2-85 \mu \delta}\right| \Im \varphi \mid
$$

Corollary 3.17. If $\varphi$ is real and $\left|\varphi-\varphi_{0}\right| \leq 2 k^{-2-40 \mu \delta}$, then $H^{j, s}(\vec{k}(\varphi))$ is monotonous in $\varphi$ and its eigenvalues $\lambda_{n}(\varphi)$ satisfy the estimates:

$$
\left|\frac{\partial \lambda_{n}(\varphi)}{\partial \varphi}\right| \geq k^{2-85 \mu \delta}
$$

Proof of the corollary. Since $\left|\vec{k}(\varphi)+\vec{p}_{\mathbf{n}}\right|_{\mathbb{R}}^{2}$ is a holomorphic function of $\varphi$ in the rectangle $\left|\Re \varphi-\varphi_{0}\right| \leq 2 k^{-2-40 \mu \delta}$, the inequality (85) yields that

$$
\left|\frac{\partial\left|\vec{k}(\varphi)+\vec{p}_{\mathbf{n}}\right|_{\mathbb{R}}^{2}}{\partial \varphi}\right| \geq k^{2-85 \mu \delta}
$$


for real $\varphi$ and the derivative has the same sign for all $\mathbf{n} \in \mathcal{M}_{2}^{j, s}$. Now, the obvious relation $\frac{\partial H^{j, s}}{\partial \varphi}=\frac{\partial H_{0}^{j, s}}{\partial \varphi}$ yields $(\underline{86})$.

Proof. It is enough to consider the case $\Im \varphi>0$. The other case is just the complex conjugated. Let $\mathbf{m} \in \mathcal{M}_{2}^{j}$. Obviously,

$$
\Im\left|\vec{k}(\varphi)+\vec{p}_{\mathbf{m}}\right|_{\mathbb{R}}^{2}=\Im 2\left(\vec{k}(\varphi), \vec{p}_{\mathbf{m}}\right)=-2 k p_{\mathbf{m}} \sin \left(\Re \varphi-\varphi_{\mathbf{m}}\right) \sinh (\Im \varphi),
$$

where $\varphi_{\mathbf{m}}$ is the angle corresponding to $\vec{p}_{\mathbf{m}}$. It suffices to show that $p_{\mathbf{m}}>k^{1-42 \mu \delta}$ and

$$
\left|\sin \left(\Re \varphi-\varphi_{\mathbf{m}}\right)\right|>k^{-42 \mu \delta} .
$$

Indeed, let $\vec{p}_{\mathbf{q}}$ be a vector, such that $0<\left\|\left|\vec{p}_{\mathbf{q}} \|\right|<k^{\delta}\right.$ and

$$
|| \vec{k}\left(\varphi_{0}\right)+\left.\vec{p}_{\mathbf{m}}\right|_{\mathbb{R}} ^{2}-k^{2}\left|\leq k^{\delta_{*}}, \quad\right|\left|\vec{k}\left(\varphi_{0}\right)+\vec{p}_{\mathbf{m}+\mathbf{q}}\right|_{\mathbb{R}}^{2}-k^{2} \mid \leq k^{\delta_{*}} .
$$

Such a $\vec{p}_{\mathbf{q}}$ exists, since $\mathbf{m} \in \mathcal{M}_{2}^{j}$. It follows:

$$
2\left|\left(\vec{k}\left(\varphi_{0}\right)+\vec{p}_{\mathbf{m}}, \vec{p}_{\mathbf{q}}\right)\right| \leq 2 k^{\delta_{*}}+(2 \pi)^{2} k^{2 \delta}
$$

Since $\varphi_{0} \in \mathcal{W}^{(1)}(k, \tau)$, we have $\left|\left(\vec{k}\left(\varphi_{0}\right), \vec{p}_{\mathbf{q}}\right)\right|>\frac{1}{8} k^{1-40 \mu \delta}$, see Lemma 3.6 for $\mathbf{m}=\mathbf{q}$. Using the last two inequalities, we obtain $\left|\left(\vec{p}_{\mathbf{m}}, \vec{p}_{\mathbf{q}}\right)\right|>\frac{1}{16} k^{1-40 \mu \delta}$. Hence, $p_{\mathbf{m}}>k^{1-42 \mu \delta}$. It remains to prove (88). Assume that $\left|\sin \left(\Re \varphi-\varphi_{\mathbf{m}}\right)\right| \leq k^{-42 \mu \delta}$. It follows from the assumption $\left|\Re \varphi-\varphi_{0}\right|<2 k^{-2-40 \mu \delta}$ that

$$
\left|\sin \left(\varphi_{0}-\varphi_{\mathbf{m}}\right)\right| \leq 2 k^{-42 \mu \delta}
$$

Since $\mathbf{m} \in \mathcal{M}_{2}^{j}$,

$$
|| \vec{k}\left(\varphi_{0}\right)+\left.\vec{p}_{\mathbf{m}}\right|_{\mathbb{R}} ^{2}-k^{2}|=| 2 k p_{\mathbf{m}} \cos \left(\varphi_{0}-\varphi_{\mathbf{m}}\right)+p_{\mathbf{m}}^{2} \mid \leq k^{\delta_{*}} .
$$

This and (90) yield $\vec{p}_{\mathbf{m}}=-2 \vec{k}\left(\varphi_{0}\right)+O\left(k^{1-42 \mu \delta}\right)$. Then,

$$
\left|2\left(\vec{k}\left(\varphi_{0}\right)+\vec{p}_{\mathbf{m}}, \vec{p}_{\mathbf{q}}\right)+p_{\mathbf{q}}^{2}\right|=\left|\left(\vec{k}\left(\varphi_{0}\right), \vec{p}_{\mathbf{q}}\right)\right|+O\left(k^{1-41 \mu \delta}\right) \geq \frac{1}{16} k^{1-40 \mu \delta}
$$

This contradicts to (89)). The contradiction completes the proof of (88). Assume $\mathcal{M}_{2}^{j}$ is trivial. Then, $\mathcal{M}_{2}^{j, s}$ contains just one point and the statement of the lemma follows immediately. If $\mathcal{M}_{2}^{j}$ is non-trivial, then considering as above, we obtain (85) for every $\mathbf{n} \in \mathcal{M}_{2}^{j, s}$. It remains to prove only that $\Im\left|\vec{k}(\varphi)+\vec{p}_{\mathbf{n}}\right|_{\mathbb{R}}^{2}$ has the same sign for all $\mathbf{n} \in \mathcal{M}_{2}^{j, s}$. By the definition of $\mathcal{M}_{2}^{j, s}$, every $\mathbf{n} \in \mathcal{M}_{2}^{j, s}$ can be written as $\mathbf{n}=\mathbf{m}_{j, s}+n \mathbf{q},|n|=O\left(k^{\delta_{*} / 2}\right)$ (see (79)). Next,

$$
\Im\left|\vec{k}(\varphi)+\vec{p}_{\mathbf{m}_{j, s}+n \mathbf{q}}\right|_{\mathbb{R}}^{2}=\Im 2\left(\vec{k}(\varphi), \vec{p}_{\mathbf{m}_{j, s}+n \mathbf{q}}\right)=-2 k p_{\mathbf{m}_{j, s}+n \mathbf{q}} \sin \left(\Re \varphi-\varphi_{\mathbf{m}_{j, s}+n \mathbf{q}}\right) \sinh (\Im \varphi),
$$


where $\varphi_{\mathbf{m}_{j, s}+n \mathbf{q}}$ is the angle corresponding to $\vec{p}_{\mathbf{m}_{j, s}+n \mathbf{q}}$. Since $|n| p_{\mathbf{q}} \leq C(Q) k^{\delta_{*} / 2}$ and $p_{\mathbf{m}_{j, s}} \geq k^{1-42 \mu \delta}$, we get $\varphi_{\mathbf{m}_{j, s}+n \mathbf{q}}=\varphi_{\mathbf{m}_{j, s}}+O\left(k^{-1+42 \mu \delta+\delta_{*} / 2}\right)$. Thus,

$$
\Im\left|\vec{k}(\varphi)+\vec{p}_{\mathbf{m}_{j, s}+n \mathbf{q}}\right|_{\mathbb{R}}^{2}=-2 k p_{\mathbf{m}_{j, s}+n \mathbf{q}}\left(\sin \left(\Re \varphi-\varphi_{\mathbf{m}_{j, s}}\right)+O\left(k^{-1+42 \mu \delta+\delta_{*} / 2}\right)\right) \sinh (\Im \varphi) .
$$

Using (88), we obtain that the right-hand side of the last inequality has the same sign for all $n: \mathbf{m}_{j, s}+n \mathbf{q} \in \mathcal{M}_{2}^{j, s}$.

Lemma 3.18. For every $j$ and $s$ the resolvent $\left(H^{j, s}(\vec{k}(\varphi))-k^{2}\right)^{-1}$ has no more than two poles in the strip $\left\{\varphi:\left|\Re \varphi-\varphi_{0}\right| \leq 2 k^{-2-40 \mu \delta},|\Im \varphi|<1\right\}$, counting multiplicity 1 . The poles are situated on the real axis.

Proof. If $\mathcal{M}_{2}^{j, s}$ consists of just one element $\mathbf{m}$, then $H^{j, s}$ has one matrix element and

$$
H_{\mathbf{m m}}^{j, s}-k^{2}=\left|\vec{k}(\varphi)+\vec{p}_{\mathbf{m}}\right|_{\mathbb{R}}^{2}-k^{2}=2 k p_{\mathbf{m}} \cos \left(\varphi-\varphi_{\mathbf{m}}\right)+p_{\mathbf{m}}^{2} .
$$

Obviously, the number of roots of this function in the strip does not exceed two. By (85), they are both real. Assume that $\mathcal{M}_{2}^{j, s}$ contains more than one element (the case of a non-trivial cluster). Obviously, $H^{j, s}-\left(H^{j, s}\right)^{*}=H_{0}^{j, s}-\left(H_{0}^{j, s}\right)^{*}$. Considering now (85), we conclude that the resolvent $\left(H^{j, s}(\vec{k}(\varphi))-k^{2}\right)^{-1}$ may have only real poles $\varphi$. The poles are described by equations $\lambda_{n}(\varphi)=k^{2}, n=1, \ldots$, the eigenvalues $\lambda_{n}$ of $H^{j, s}$ being numerated in the increasing order counting multiplicity. By Corollary 3.17. $\partial \lambda_{n} / \partial \varphi \neq 0$ and the derivatives have the same sign for all $n$. Assume, for definiteness, that $\partial \lambda_{n} / \partial \varphi>0$. It follows that the order of each pole $\varphi_{k}$ of the resolvent is less or equal to the multiplicity of the corresponding eigenvalue $\lambda_{n(k)}\left(\varphi_{k}\right)$. If for two poles we have $\varphi_{k}<\varphi_{k^{\prime}}$, then $n\left(k^{\prime}\right)<n(k)$ because of monotonicity of $\lambda_{n}(\varphi)$. Thus, the number of poles $\varphi_{k}$ (counting multiplicity) in the disk $\left|\varphi-\varphi_{0}\right| \leq 2 k^{-2-40 \mu \delta}$ does not exceed the number of eigenvalues (counting multiplicity) satisfying $\lambda_{n}(\varphi)=k^{2}$ for some $\varphi \in \mathbb{R}:\left|\varphi-\varphi_{0}\right| \leq 2 k^{-2-40 \mu \delta}$. Note that by perturbation theory it is enough to estimate the number of eigenvalues of $H^{j, s}\left(\vec{k}\left(\varphi_{0}\right)\right)$ in the interval $\left[k^{2}-4 k^{-40 \mu \delta}, k^{2}+4 k^{-40 \mu \delta}\right]$.

Using the "separation of the variables" (라), we rewrite $\left(H^{j, s}(\vec{k}(\varphi))-k^{2}\right)^{-1}$ in the form $\left(\tilde{H}^{j, s}\left(t_{\mathbf{q}}\right)+\left(t_{\mathbf{q}}^{\perp}\right)^{2}-k^{2}\right)^{-1}$. We denote eigenvalues of $\tilde{H}^{j, s}\left(t_{\mathbf{q}}\right)$ by $\tilde{\lambda}_{n}\left(t_{\mathbf{q}}\right)$. Thus, $\lambda_{n}(\varphi)=$ $\tilde{\lambda}_{n}\left(t_{\mathbf{q}}\right)+\left(t_{\mathbf{q}}^{\perp}\right)^{2}$. It suffices to prove that there are no more than two eigenvalues $\tilde{\lambda}_{n}\left(t_{\mathbf{q}}\right)$ (counting multiplicity) in the interval $\left[k^{2}-\left(t_{\mathbf{q}}^{\perp}\right)^{2}-4 k^{-40 \mu \delta}, k^{2}-\left(t_{\mathbf{q}}^{\perp}\right)^{2}+4 k^{-40 \mu \delta}\right]$. Suppose there are at least three. For a non-trivial cluster, $\left|k^{2}-\left(t_{\mathbf{q}}^{\perp}\right)^{2}\right| \leq \frac{1}{8} k^{\delta_{*}}$ at $\varphi_{0}$, see Definition 3.15. It follows from the defnition of $\mathcal{M}_{2}^{j, s}$ that all $n$ such that $\left(t_{\mathbf{q}}+n p_{\mathbf{q}}\right)^{2} \leq \frac{7}{8} k^{\delta_{*}}$ are present in the matrix of $\tilde{H}^{j, s}\left(t_{\mathbf{q}}\right)$. Now, by the perturbation theory, all eigenvalues of $\tilde{H}^{j, s}\left(t_{\mathbf{q}}\right)$ such that $\left|\tilde{\lambda}_{n}\left(t_{\mathbf{q}}\right)\right| \leq \frac{1}{8} k^{\delta_{*}}$ can be approximated by eigenvalues of the corresponding infinite matrix $\tilde{H}_{\text {per }}^{j, s}\left(t_{\mathbf{q}}\right)$ (cf. ( $(\underline{83})$ ) with an accuracy $O\left(k^{-\frac{\delta_{*}}{C(Q)} k^{\delta_{*} / 2}}\right)$. Infinite matrix $\tilde{H}_{\text {per }}^{j, s}\left(t_{\mathbf{q}}\right)$

\footnotetext{
${ }^{1}$ By the multiplicity of a pole of the resolvent we mean the multiplicity of the zero of $\operatorname{det}\left(H^{j, s}-k^{2}\right)$ as function of $\varphi$.
} 
corresponds to the one-dimensional periodic Schrödinger operator with the period $p_{\mathbf{q}}^{-1}$. We denote the eigenvalues of the matrix $\tilde{H}_{\text {per }}^{j, s}\left(t_{\mathbf{q}}\right)$ by $\tilde{\lambda}_{n}^{\text {per }}\left(t_{\mathbf{q}}\right)$. We have

$$
\tilde{\lambda}_{n}^{p e r}\left(t_{\mathbf{q}}\right)=\tilde{\lambda}_{n}\left(t_{\mathbf{q}}\right)+O\left(k^{-\frac{\delta_{*}}{C(Q)} k^{\delta_{*} / 2}}\right) .
$$

Thus, if the operator $H^{j, s}\left(\vec{k}\left(\varphi_{0}\right)\right)$ has three eigenvalues in the interval $\left[k^{2}-4 k^{-40 \mu \delta}, k^{2}+\right.$ $\left.4 k^{-40 \mu \delta}\right]$, then $\tilde{H}_{\text {per }}^{j, s}\left(t_{\mathbf{q}}\right)$ has at least three eigenvalues in the interval $\left[k^{2}-\left(t_{\mathbf{q}}^{\perp}\right)^{2}-8 k^{-40 \mu \delta}, k^{2}-\right.$ $\left.\left(t_{\mathbf{q}}^{\perp}\right)^{2}+8 k^{-40 \mu \delta}\right]$. Since every eigenvalue belongs to a different zone of the corresponding one-dimensional periodic operator, it means that there is a zone in the spectrum with the length less than $16 k^{-40 \mu \delta}$. We also notice that $t_{\mathbf{q}}$ in the matrix $\tilde{H}_{p e r}^{j, s}\left(t_{\mathbf{q}}\right)$ plays the role of quasi-momentum, while the corresponding one-dimensional periodic operator does not depend on $k$. Now, we arrive at the contradiction if $k^{-40 \mu \delta}$ is sufficiently small (obviously, depending on $V$ only).

Definition 3.19. We call a set $\mathcal{M}_{2}^{j, s}$ strongly resonant if the corresponding resolvent $\left(H^{j, s}(\vec{k}(\varphi))-k^{2}\right)^{-1}$ has at least one pole in the disk $\left|\varphi-\varphi_{0}\right| \leq 2 k^{-2-40 \mu \delta}$. Otherwise the set is called weakly resonant.

We say that two strongly resonant sets $\mathcal{M}_{2}^{j, s}$ and $\mathcal{M}_{2}^{j^{\prime}, s^{\prime}}$ are adjacent, if the $\|\mid \cdot\| \|$ distance between them is no more than $3 k^{\delta}$. We say that two sets $\mathcal{M}_{2}^{j, s}$ and $\mathcal{M}_{2}^{j, s^{\prime}}$ belong to the same cluster if they can be connected via some path of subsequently adjacent sets. This defines an equivalence relation. Thus, strongly resonant sets $\mathcal{M}_{2}^{j, s}$ form clusters (equivalence classes). The cluster, containing a strongly resonant set $\mathcal{M}_{2}^{j, s}$, we denote by $\mathcal{M}_{2, s t r}^{j, s}$. Obviously, $\mathcal{M}_{2, s t r}^{j, s}=\mathcal{M}_{2, s t r}^{j, s^{\prime}}$ when $\mathcal{M}_{2}^{j, s}$ and $\mathcal{M}_{2}^{j, s^{\prime}}$ are from the same cluster.

Lemma 3.20. A cluster $\mathcal{M}_{2, \text { str }}^{j, s}$ of strongly resonant sets contains no more than two sets $\mathcal{M}_{2}^{j, s}$.

Proof. By the definition of $\mathcal{M}_{2}^{j}$, all $\mathcal{M}_{2}^{j, s}$ in a cluster belong to the same $\mathcal{M}_{2}^{j}$. First, assume $\mathcal{M}_{2}^{j}$ is trivial. Then, by definition, each $\mathcal{M}_{2}^{j, s}$ consists of just one point $\mathbf{m}$ and, by the Definition 3.19, ||$\vec{k}\left(\varphi_{0}\right)+\left.\vec{p}_{\mathbf{m}}\right|_{\mathbb{R}} ^{2}-k^{2} \mid=O\left(k^{-40 \mu \delta}\right)$. Suppose there are more than two $\mathcal{M}_{2}^{j, s}$ in the cluster. Then, there are $\mathbf{n}, \mathbf{n}+\mathbf{q}_{1}, \mathbf{n}+\mathbf{q}_{2} \in \mathcal{M}_{2}^{j}$ such that $\mathbf{q}_{1} \neq \mathbf{q}_{2}$, $0<\left\|\left|\vec{p}_{\mathbf{q}_{1}}\left\|\left|,\left\|\left|\vec{p}_{\mathbf{q}_{2}} \|\right|<3 k^{\delta}\right.\right.\right.\right.\right.$, and

$$
|| \vec{k}\left(\varphi_{0}\right)+\left.\vec{p}_{\mathbf{n}}\right|_{\mathbb{R}} ^{2}-k^{2}\left|=O\left(k^{-40 \mu \delta}\right), \quad\right|\left|\vec{k}\left(\varphi_{0}\right)+\vec{p}_{\mathbf{n}+\mathbf{q}_{i}}\right|_{\mathbb{R}}^{2}-k^{2} \mid=O\left(k^{-40 \mu \delta}\right), i=1,2 .
$$

It follows that

$$
\left|2\left(\vec{k}\left(\varphi_{0}\right)+\vec{p}_{\mathbf{n}}, \vec{p}_{\mathbf{q}_{i}}\right)+p_{\mathbf{q}_{i}}^{2}\right|=O\left(k^{-40 \mu \delta}\right), \quad i=1,2 .
$$

By Lemma 3.13, $\mathbf{q}_{1}$ is parallel to $\mathbf{q}_{2}$. Now (94) yields $p_{\mathbf{q}_{1}-\mathbf{q}_{2}}=\left|p_{\mathbf{q}_{1}}-p_{\mathbf{q}_{2}}\right|=O\left(k^{-38 \mu \delta}\right)$. Using (30), we obtain $\mathbf{q}_{1}=\mathbf{q}_{2}$, since $\left\|\left|\vec{p}_{\mathbf{q}_{1}}\left\|\left|,\left\|\left|\vec{p}_{\mathbf{q}_{2}} \|\right|<3 k^{\delta}\right.\right.\right.\right.\right.$. However, $\mathbf{q}_{1} \neq \mathbf{q}_{2}$ by construction. Thus, the lemma is proven in the case of a trivial $\mathcal{M}_{2}^{j}$. 
Suppose $\mathcal{M}_{2}^{j}$ is not trivial and $\vec{p}_{\mathbf{q}}$ is its directional vector, $\mathbf{q} \in \mathcal{S}_{Q}$. Let $\mathcal{M}_{2}^{j, s_{i}}, i=$ $0,1,2$, be any three neighboring elements in a $3 k^{\delta}$-cluster. Let $\mathbf{m}_{i}$ be the central point of $\mathcal{M}_{2}^{j, s_{i}}, i=0,1,2$. All $\mathcal{M}_{2}^{j, s_{i}}$ are subsets of periodic one-dimensional lattices with the same period $\vec{p}_{\mathbf{q}}$. By Corollary $\underline{3.14}, \vec{p}_{\mathbf{m}_{0}-\mathbf{m}_{i}}=w_{i} \vec{p}_{\mathbf{q}}, w_{i} \in \mathbb{R}, i=1,2$. Since $\mathbf{m}_{i}$ are central points, we have $\left|w_{i}\right|<1$, see (179). Let us show that at least one of the numbers $w_{1}, w_{2}, w_{1}-w_{2}$ is an integer. Suppose it is not so. By Definition 3.19 and elementary perturbation consideration, each operator $H^{j, s_{i}}\left(\vec{k}\left(\varphi_{0}\right)\right)$ has an eigenvalue $\lambda_{n_{i}}^{\left(s_{i}\right)}\left(\varphi_{0}\right)$ such that $\lambda_{n_{i}}^{\left(s_{i}\right)}\left(\varphi_{0}\right)=k^{2}+O\left(k^{-40 \mu \delta}\right)$. Using (82), we obtain:

$$
\tilde{\lambda}_{n_{i}}\left(t_{\mathbf{q}}^{\left(s_{i}\right)}\right)+\left(t_{\mathbf{q}}^{\perp}\right)^{2}=k^{2}+O\left(k^{-40 \mu \delta}\right)
$$

where $\tilde{\lambda}_{n_{i}}\left(t_{\mathbf{q}}^{\left(s_{i}\right)}\right)$ is an eigenvalue of $\tilde{H}^{j, s_{i}}$. By (93) , these eigenvalues of $\tilde{H}^{j, s_{i}}, i=0,1,2$, can be approximated by the corresponding eigenvalues of $\tilde{H}_{p e r}^{j, s}$ with the high accuracy $O\left(k^{-\frac{\delta_{*}}{C(Q)} k^{\delta_{*} / 2}}\right)$. It follows from (95) that

$$
\begin{gathered}
\left|\tilde{\lambda}_{n_{0}}^{\text {per }}\left(t_{\mathbf{q}}^{\left(s_{0}\right)}\right)-\tilde{\lambda}_{n_{1}}^{\text {per }}\left(t_{\mathbf{q}}^{\left(s_{1}\right)}\right)\right|=O\left(k^{-40 \mu \delta}\right), \\
\left|\tilde{\lambda}_{n_{0}}^{\text {per }}\left(t_{\mathbf{q}}^{\left(s_{0}\right)}\right)-\tilde{\lambda}_{n_{2}}^{\text {per }}\left(t_{\mathbf{q}}^{\left(s_{2}\right)}\right)\right|=O\left(k^{-40 \mu \delta}\right) .
\end{gathered}
$$

All $\tilde{\lambda}_{n_{i}}^{\text {per }}\left(t_{\mathbf{q}}^{\left(s_{i}\right)}\right), i=0,1,2$, are eigenvalues of the same fiber matrix $\tilde{H}_{\text {per }}^{j}$, but at different values of the quasi-momentum $t_{\mathbf{q}}^{\left(s_{i}\right)}$ (see (84)). Recall that the corresponding periodic operator does not depend on $k$. Properties of the one-dimensional periodic Schrödinger operators (i.e. non-degeneracy of the band functions) and (96), imply that for sufficiently large $k$, depending on $V$ only:

$$
\left|\left(t_{\mathbf{q}}^{\left(s_{0}\right)} \pm t_{\mathbf{q}}^{\left(s_{i}\right)}\right)_{\bmod p_{\mathbf{q}}}\right|=O\left(k^{-20 \mu \delta}\right), \quad i=1,2
$$

Substituting the expression for $t_{\mathbf{q}}($ see (84) $)$ into the above estimate and considering that $\vec{p}_{\mathbf{m}_{0}-\mathbf{m}_{i}}=w_{i} \vec{p}_{\mathbf{q}}, i=1,2$, yields that either $\left|w_{i}-n_{i}\right|=O\left(k^{-20 \mu \delta}\right)$ or $\left|2 t_{\mathbf{q}}^{\left(s_{0}\right)} p_{\mathbf{q}}^{-1}-w_{i}-n_{i}\right|=$ $O\left(k^{-20 \mu \delta}\right)$ for some $n_{i} \in \mathbb{Z}, i=1,2$. Suppose the first relation holds for $i=1$. By properties of $w_{1}, p_{\mathbf{m}_{0}-\mathbf{m}_{1}-n_{1} \mathbf{q}}=O\left(k^{-20 \mu \delta}\right)$. Let us estimate $\left\|\left|\vec{p}_{\mathbf{m}_{0}-\mathbf{m}_{1}-n_{1} \mathbf{q}} \|\right|\right.$ from above. Since $\mathbf{m}_{0}$ and $\mathbf{m}_{1}$ are central points of adjacent sets $\mathcal{M}_{2}^{j, s_{i}}$, we have $\left\|\left|\vec{p}_{\mathbf{m}_{0}-\mathbf{m}_{1}} \|\right|<3 C(Q) k^{\delta}\right.$, see Appendix 3. Since $\left|w_{1}\right|<1$, and $\left|w_{1}-n_{1}\right|=O\left(k^{-20 \mu \delta}\right)$, we have $\left|n_{1}\right|<2$. It follows that $\left\|\left|\vec{p}_{\mathbf{m}_{0}-\mathbf{m}_{1}-n_{1} \mathbf{q}} \|\right|=O\left(k^{\delta}\right)\right.$. Therefore, by (30), $p_{\mathbf{m}_{0}-\mathbf{m}_{1}-n_{1} \mathbf{q}}>k^{-2 \mu \delta}$. This contradicts to the inequality $p_{\mathbf{m}_{0}-\mathbf{m}_{1}-n_{1} \mathbf{q}}=O\left(k^{-20 \mu \delta}\right)$ obtained just above. Therefore, $\mathbf{m}_{0}-\mathbf{m}_{1}-n_{1} \mathbf{q}=\mathbf{0}$, that is $w_{1}=n_{1}$. This contradicts to the initial assumption that $w_{1}$ is not an integer. If $\left|w_{2}-n_{2}\right|=O\left(k^{-20 \mu \delta}\right)$, we arrive to the the analogous contradiction for $w_{2}$. It remains to assume that $\left|2 t_{\mathbf{q}}^{\left(s_{0}\right)} p_{\mathbf{q}}^{-1}-w_{i}-n_{i}\right|=O\left(k^{-20 \mu \delta}\right)$ for both $i=1$, 2. It follows $\left|w_{1}-w_{2}-\left(n_{2}-n_{1}\right)\right|=O\left(k^{-20 \mu \delta}\right)$ and, hence, $p_{\mathbf{m}_{1}-\mathbf{m}_{2}-n \mathbf{q}}=O\left(k^{-20 \mu \delta}\right), n=n_{2}-n_{1}$. Let us estimate $\left\|\left|\vec{p}_{\mathbf{m}_{1}-\mathbf{m}_{2}-n \mathbf{q}} \|\right|\right.$ from above. Since $\mathbf{m}_{1}$ and $\mathbf{m}_{2}$ are central points, we have $\left\|\left|\vec{p}_{\mathbf{m}_{1}-\mathbf{m}_{2}} \|\right|<6 C(Q) k^{\delta}\right.$, see Appendix 3. Since $\left|w_{1}-w_{2}-\left(n_{2}-n_{1}\right)\right|=O\left(k^{-20 \mu \delta}\right)$, we have $|n|<3$. It follows that $\left\|\left|\vec{p}_{\mathbf{m}_{1}-\mathbf{m}_{2}-n \mathbf{q}} \|\right|=O\left(k^{\delta}\right)\right.$. Therefore, by (130), $p_{\mathbf{m}_{1}-\mathbf{m}_{2}-n \mathbf{q}}>k^{-2 \mu \delta}$. 
This contradicts to the inequality $p_{\mathbf{m}_{1}-\mathbf{m}_{2}-n \mathbf{q}}=O\left(k^{-20 \mu \delta}\right)$ obtained just above. Hence, we obtain $w_{1}-w_{2}=n$. Thus, we proved that at least two out of each three neighboring sets $\mathcal{M}_{2}^{j, s}$ in a cluster are shifted with respect to each other by $n \vec{p}_{\mathbf{q}}, n \in \mathbb{Z}$, and, thus, (see (79) ) they coincide. Moreover, we proved that if $w_{i}$ is not an integer, then it is equal to $2 t_{\mathbf{q}}^{\left(s_{0}\right)} p_{\mathbf{q}}^{-1}+n_{i}, n_{i} \in \mathbb{Z}$.

\subsubsection{Estimates for the Resolvent of the Model Operator}

We are going to obtain estimates for the resolvent $\left(P\left(r_{1}\right)\left(H\left(\vec{\varkappa}^{(1)}(\varphi)\right)-k^{2}\right) P\left(r_{1}\right)\right)^{-1}$. We start with the following lemma.

Lemma 3.21. Let $\left|\varphi-\varphi_{0}\right| \leq k^{-2}$. If $\mathbf{m}$ does not belong to $\mathcal{M}\left(\varphi_{0}\right)$ then

$$
\left|\left(H_{0}(\vec{k}(\varphi))-k^{2}\right)_{\mathbf{m m}}\right| \geq \frac{1}{2} k^{\delta_{*}}, \quad\left|\left(H_{0}\left(\vec{\varkappa}^{(1)}(\varphi)\right)-k^{2}\right)_{\mathbf{m m}}\right| \geq \frac{1}{2} k^{\delta_{*}} .
$$

Proof. The proof follows from the definition of $\mathcal{M}\left(\varphi_{0}\right)$ (see (74), (72)), (68) and simple perturbative arguments.

Let $P_{\mathbf{m}}$ be the orthogonal projector corresponding to the set $\tilde{\mathcal{M}}_{\mathbf{m}}$, see (75). We consider $\mathbf{m} \in \mathcal{M}_{1}$. The following lemma is an analogue of Lemma 3.14 from [1] (statements 1-3). The proof is very similar, however, we present it here for completeness. The statement 4 of Lemma 3.14 from [1] is now quite different and will require additional arguments which we present below. It is the main place where we have the difference between the Schrödinger operator and polyharmonic operators considered in [1].

Lemma 3.22. Let $\varphi_{0} \in \omega^{(1)}(k, 8)$.

1. If $\mathbf{m} \in \mathcal{M}_{1}\left(\varphi_{0}\right): p_{\mathbf{m}}>4 k^{\delta},\left|2 k-p_{\mathbf{m}}\right| \geq 1$, then, the operator

$$
\left(P_{\mathbf{m}}\left(H\left(\vec{\varkappa}^{(1)}(\varphi)\right)-k^{2} I\right) P_{\mathbf{m}}\right)^{-1}
$$

has no more than one pole in the disk $\left|\varphi-\varphi_{0}\right|<2 k^{-2-\delta(40 \mu+1)}$. The following estimate holds:

$$
\left\|\left(P_{\mathbf{m}}\left(H\left(\vec{\varkappa}^{(1)}(\varphi)\right)-k^{2} I\right) P_{\mathbf{m}}\right)^{-1}\right\|<c k^{-1} \varepsilon_{0}^{-1}, \quad \varepsilon_{0}=\min \left\{\varepsilon, k^{-2-(40 \mu+1) \delta}\right\},
$$

when $\varphi$ is in the smaller disk $\left|\varphi-\varphi_{0}\right|<k^{-2-\delta(40 \mu+1)}$, $\varepsilon$ being the distance from $\varphi$ to the nearest pole of the operator.

2. If $\mathbf{m} \in \mathcal{M}:\left|2 k-p_{\mathbf{m}}\right|<1$, then, in fact, $\mathbf{m} \in \mathcal{M}_{1}$ and the operator

$$
\left(P_{\mathbf{m}}\left(H\left(\vec{\varkappa}^{(1)}(\varphi)\right)-k^{2} I\right) P_{\mathbf{m}}\right)^{-1}
$$


has no more than two poles in the disk $\left|\varphi-\varphi_{0}\right|<2 k^{-2-\delta(40 \mu+1)}$. The following estimate holds:

$$
\left\|\left(P_{\mathbf{m}}\left(H\left(\vec{\varkappa}^{(1)}(\varphi)\right)-k^{2} I\right) P_{\mathbf{m}}\right)^{-1}\right\|<c k^{-2} \varepsilon_{0}^{-2}, \quad \varepsilon_{0}=\min \left\{\varepsilon, k^{-2-\delta(40 \mu+1)}\right\},
$$

when $\varphi$ is in the smaller disk $\left|\varphi-\varphi_{0}\right|<k^{-2-\delta(40 \mu+1)}$, $\varepsilon$ being the distance from $\varphi$ to the nearest pole of the operator.

3. If $\mathbf{m} \in \mathcal{M}: p_{\mathbf{m}}<4 k^{\delta}$, then, in fact, $\mathbf{m} \in \mathcal{M}_{1}$ and the operator

$$
\left(P_{\mathbf{m}}\left(H\left(\vec{\varkappa}^{(1)}(\varphi)\right)-k^{2} I\right) P_{\mathbf{m}}\right)^{-1}
$$

has no more than one pole in the disk $\left|\varphi-\varphi_{0}\right|<2 k^{-2-\delta(40 \mu+1)}$. The following estimate holds:

$$
\left\|\left(P_{\mathbf{m}}\left(H\left(\vec{\varkappa}^{(1)}(\varphi)\right)-k^{2} I\right) P_{\mathbf{m}}\right)^{-1}\right\|<8 k^{-1} p_{\mathbf{m}}^{-1} \varepsilon_{0}^{-1}, \quad \varepsilon_{0}=\min \left\{\varepsilon, k^{-1+\delta}\right\},
$$

when $\varphi$ is in the smaller disk $\left|\varphi-\varphi_{0}\right|<k^{-2-\delta(40 \mu+1)}$, $\varepsilon$ being the distance from $\varphi$ to the nearest pole of the operator.

Proof.

1. Let $\left|2 k-p_{\mathbf{m}}\right| \geq 1, p_{\mathbf{m}}>4 k^{\delta}$. Clearly only the case $p_{\mathbf{m}}<4 k$ is significant, since otherwise $\mathcal{O}_{\mathbf{m}}(k, 1)$ cannot intersect the disc $\left|\varphi-\varphi_{0}\right|<k^{-2-40(\mu+1) \delta}$ by Lemma 3.12, It is easy to see that the set $\mathcal{O}_{\mathbf{m}}(k, 1)$ consists of two separate $\operatorname{discs} \mathcal{O}_{\mathbf{m}}^{ \pm}(k, 1)$, the distance between them being greater than $c k^{-1 / 2}$. Let us assume for definiteness $\varphi_{0} \in \mathcal{O}_{\mathbf{m}}^{+}(k, 1)$. This means the disc $\left|\varphi-\varphi_{0}\right|<k^{-2-\delta(40 \mu+1)}$ does not intersect $\mathcal{O}_{\mathbf{m}}^{-}(k, 1)$. Let us first show that the operator

$$
\left(P_{\mathbf{m}}\left(H_{0}\left(\vec{\varkappa}^{(1)}(\varphi)\right)-k^{2} I\right) P_{\mathbf{m}}\right)^{-1}
$$

has exactly one pole inside $\mathcal{O}_{\mathbf{m}}^{+}(k, 1)$, which is, in fact, inside $\mathcal{O}_{\mathbf{m}}^{+}(k, 1 / 4)$. Note that $\vec{\varkappa}^{(1)}(\varphi)$ is defined in $\mathcal{O}_{\mathbf{m}}^{+}(k, 1)$, since the size of $\mathcal{O}_{\mathbf{m}}^{+}(k, 1)$ is much less than that of any circle in $\mathcal{O}^{(1)}$. It satisfies the estimate $\vec{\varkappa}^{(1)}(\varphi)=\vec{k}(\varphi)+o\left(k^{-2}\right)$ in $\mathcal{O}_{\mathbf{m}}^{+}$, see (68). If $\varphi_{0} \in \mathcal{O}_{\mathbf{m}}^{+}(k, 1) \backslash \mathcal{O}_{\mathbf{m}}^{+}(k, 1 / 4)$, then the estimates ||$\vec{\varkappa}^{(1)}\left(\varphi_{0}\right)+\left.\vec{p}_{\mathbf{m}+\mathbf{q}}\right|^{2}-k^{2} \mid>\frac{1}{4} k^{\delta_{*}}$, hold for $0 \leq\left\|\left|\vec{p}_{\mathbf{q}} \|\right|<k^{\delta}\right.$ (see the definition of $\mathcal{M}_{1}\left(\varphi_{0}\right)$ ) and these estimates can be extended to the $\left(k^{-2-(40 \mu+1) \delta}\right)$-neighborhood of $\varphi_{0}\left(\frac{1}{4}\right.$ becomes $\left.\frac{1}{8}\right)$. Thus,

$$
\left\|\left(P_{\mathbf{m}}\left(H_{0}\left(\vec{\varkappa}^{(1)}(\varphi)\right)-k^{2} I\right) P_{\mathbf{m}}\right)^{-1}\right\|<c k^{-\delta_{*}}
$$

when $\left|\varphi-\varphi_{0}\right|<k^{-2-(40 \mu+1) \delta}, \quad \varphi_{0} \in \mathcal{O}_{\mathbf{m}}^{+}(k, 1) \backslash \mathcal{O}_{\mathbf{m}}^{+}(k, 1 / 4)$.

Clearly the resolvent (102) does not have poles in the set $\left|\varphi-\varphi_{0}\right|<k^{-2-(40 \mu+1) \delta}$. The estimate (98) with $\varepsilon_{0}=k^{-2-(40 \mu+1) \delta}$ follows from (102) and Hilbert identity. 
Now, suppose that $\varphi_{0} \in \mathcal{O}_{\mathbf{m}}^{+}\left(k, \frac{1}{4}\right)$. The function $\left|\vec{k}(\varphi)+\vec{p}_{\mathbf{m}}\right|_{\mathbb{R}}^{2}-k^{2}$ has a single zero inside $\mathcal{O}_{\mathbf{m}}^{+}\left(k, \frac{1}{4}\right)$. Using Rouché's Theorem, we obtain that $\left|\vec{\varkappa}^{(1)}(\varphi)+\vec{p}_{\mathbf{m}}\right|_{\mathbb{R}}^{2}-k^{2}$ also has a single zero inside $\mathcal{O}_{\mathbf{m}}^{+}\left(k, \frac{1}{4}\right)$. Note that the following inequality holds in $\mathcal{O}_{\mathbf{m}}^{+}\left(k, \frac{1}{4}\right)$ for $0<\left\|\left|\vec{p}_{\mathbf{q}} \|\right|<k^{\delta}\right.$ :

$$
|| \vec{\varkappa}^{(1)}(\varphi)+\left.\vec{p}_{\mathbf{m}+\mathbf{q}}\right|_{\mathbb{R}} ^{2}-k^{2} \mid>\frac{1}{4} k^{\delta_{*}} .
$$

Indeed, if ||$\vec{\varkappa}^{(1)}(\varphi)+\left.\vec{p}_{\mathbf{m}+\mathbf{q}}\right|_{\mathbb{R}} ^{2}-k^{2} \mid \leq \frac{1}{4} k^{\delta_{*}}$ for some $\mathbf{q} \neq(0,0)$ and $\varphi \in \mathcal{O}_{\mathbf{m}}^{+}\left(k, \frac{1}{4}\right)$, then

$$
\left|2\left(\vec{\varkappa}^{(1)}(\varphi)+\vec{p}_{\mathbf{m}}, \vec{p}_{\mathbf{q}}\right)_{\mathbb{R}}+p_{\mathbf{q}}^{2}\right|<\frac{1}{2} k^{\delta_{*}} .
$$

Considering that the size of $\mathcal{O}_{\mathbf{m}}^{+}\left(k, \frac{1}{4}\right)$ is $\frac{k^{-1+\delta_{*}}}{p_{\mathbf{m}} \sqrt{1-p_{\mathbf{m}}^{2}(2 k)^{-2}}}(1+o(1))$ and that $p_{\mathbf{m}}>$ $4 k^{\delta}>4 p_{\mathbf{q}} / 2 \pi$, we obtain the inequality analogous to (103) for $\varphi_{0}$ with $\frac{3}{4}$ instead of $\frac{1}{2}$. This contradicts to the assumption $\mathbf{m} \in \mathcal{M}_{1}\left(\varphi_{0}\right)$. Thus, the following inequality holds for all $\mathbf{q}:\left\|\vec{p}_{\mathbf{q}}\right\| \mid<k^{\delta}$ including $\mathbf{q}=(0,0)$ :

$$
|| \vec{\varkappa}^{(1)}(\varphi)+\left.\vec{p}_{\mathbf{m}+\mathbf{q}}\right|_{\mathbb{R}} ^{2}-k^{2} \mid \geq \frac{1}{4} k^{\delta_{*}}
$$

when $\varphi$ is on the boundary of $\mathcal{O}_{\mathbf{m}}^{+}\left(k, \frac{1}{4}\right)$. Hence, the resolvent

$$
\left(P_{\mathbf{m}}\left(H_{0}\left(\vec{\varkappa}^{(1)}(\varphi)\right)-k^{2} I\right) P_{\mathbf{m}}\right)^{-1}
$$

of the free operator $P_{\mathbf{m}} H_{0}$ has exactly one pole inside $\mathcal{O}_{\mathbf{m}}^{+}\left(k, \frac{1}{4}\right)$ and

$$
\left\|\left(P_{\mathbf{m}}\left(H_{0}\left(\vec{\varkappa}^{(1)}(\varphi)\right)-k^{2} I\right) P_{\mathbf{m}}\right)^{-1}\right\| \leq 4 k^{-\delta_{*}}
$$

when $\varphi$ is on the boundary on the disc $\mathcal{O}_{\mathbf{m}}^{+}\left(k, \frac{1}{4}\right)$. Considering that the dimension of $P_{\mathbf{m}}$ does not exceed $16 k^{4 \delta}$ we obtain:

$$
\left\|\left(P_{\mathbf{m}}\left(H_{0}\left(\vec{\varkappa}^{(1)}(\varphi)\right)-k^{2} I\right) P_{\mathbf{m}}\right)^{-1}\right\|_{1} \leq 64 k^{-\delta_{*}+4 \delta} .
$$

It remains to prove the analogous result for the perturbed operator $H$. We introduce the determinant

$$
D(\varphi)=\operatorname{det}\left(P_{\mathbf{m}}\left(H\left(\vec{\varkappa}^{(1)}(\varphi)\right)-k^{2} I\right) P_{\mathbf{m}}\left(H_{0}\left(\vec{\varkappa}^{(1)}(\varphi)\right)-k^{2} I\right)^{-1} P_{\mathbf{m}}\right) .
$$

Obviously, $D(\varphi)=\operatorname{det}(I+A)$, where $I, A: P_{\mathbf{m}} L_{2}\left(\mathbb{Z}^{2}\right) \rightarrow P_{\mathbf{m}} L_{2}\left(\mathbb{Z}^{2}\right)$

$$
A(\varphi)=P_{\mathbf{m}} V\left(H_{0}\left(\vec{\varkappa}^{(1)}(\varphi)\right)-k^{2} I\right)^{-1} P_{\mathbf{m}} .
$$


Taking into account that

$$
D(\varphi)=\frac{\operatorname{det}\left(P_{\mathbf{m}}\left(H\left(\vec{\varkappa}^{(1)}(\varphi)\right)-k^{2} I\right) P_{\mathbf{m}}\right)}{\operatorname{det}\left(P_{\mathbf{m}}\left(H_{0}\left(\vec{\varkappa}^{(1)}(\varphi)\right)-k^{2} I\right) P_{\mathbf{m}}\right)},
$$

we see that $D(\varphi)$ is a meromorphic function inside $\mathcal{O}_{\mathbf{m}}^{+}\left(k, \frac{1}{4}\right)$. Next, we employ a well-known inequality for the determinants, see [40]:

$$
|\operatorname{det}(I+A)-\operatorname{det}(I+B)| \leq\|A-B\|_{1} \exp \left(\|A\|_{1}+\|B\|_{1}+1\right), \quad A, B \in \mathbf{S}_{\mathbf{1}} .
$$

Putting $A=A(\varphi), B=0$, we obtain

$$
|\operatorname{det}(I+A)-1| \leq\|A\|_{1} \exp \left(\|A\|_{1}+1\right) .
$$

It is easy to see that

$$
\|A\|_{1} \leq\|V\|\left\|P_{\mathbf{m}}\left(H_{0}\left(\vec{\varkappa}^{(1)}(\varphi)\right)-k^{2} I\right)^{-1} P_{\mathbf{m}}\right\|_{1} .
$$

Considering the estimate (105) for the resolvent of the free operator, we obtain $\left\|A_{1}(\varphi)\right\|_{1}<1 / 200$ on the boundary of $\mathcal{O}_{\mathbf{m}}^{+}\left(k, \frac{1}{4}\right)$ for sufficiently large $k$. By Rouché's Theorem, $D(\varphi)$ has only one zero in $\mathcal{O}_{\mathbf{m}}^{+}\left(k, \frac{1}{4}\right)$. Thus, $\operatorname{det} P_{\mathbf{m}}\left(H\left(\vec{\varkappa}^{(1)}(\varphi)\right)-k^{2} I\right) P_{\mathbf{m}}$ has exactly one zero in $\mathcal{O}_{\mathbf{m}}^{+}\left(k, \frac{1}{4}\right)$. Using this, we immediately obtain that operator $\left(P_{\mathbf{m}}\left(H\left(\vec{\varkappa}^{(1)}(\varphi)\right)-k^{2} I\right) P_{\mathbf{m}}\right)^{-1}$ has one pole inside $\mathcal{O}_{\mathbf{m}}^{+}\left(k, \frac{1}{4}\right)$. Considering the estimate for the free resolvent and using Hilbert identity, we immediately obtain,

$$
\left\|\left(P_{\mathbf{m}}\left(H\left(\vec{\varkappa}^{(1)}(\varphi)\right)-k^{2} I\right) P_{\mathbf{m}}\right)^{-1}\right\| \leq 8 k^{-\delta_{*}}
$$

for all $\varphi$ on the boundary of $\mathcal{O}_{\mathbf{m}}^{+}\left(k, \frac{1}{4}\right)$. Taking into account that the size of $\mathcal{O}_{\mathbf{m}}^{+}\left(k, \frac{1}{4}\right)$ does not exceed $k^{-1+\delta_{*}}$, we obtain:

$$
\left\|\left(P_{\mathbf{m}}\left(H\left(\vec{\varkappa}^{(1)}(\varphi)\right)-k^{2} I\right) P_{\mathbf{m}}\right)^{-1}\right\| \leq 8 k^{-\delta_{*}}\left(k^{-1+\delta_{*}} / \varepsilon\right)
$$

when $\varphi \in \mathcal{O}_{\mathbf{m}}^{+}\left(k, \frac{1}{4}\right)$ on the distance $\varepsilon$, from the pole. If $\left|\varphi-\varphi_{0}\right|<k^{-2-(40 \mu+1) \delta}$, but $\varphi \notin \mathcal{O}_{\mathbf{m}}^{+}\left(k, \frac{1}{4}\right)$, then $\varphi$ is on the distance less than $k^{-2-(40 \mu+1) \delta}$ from the boundary of $\mathcal{O}_{\mathbf{m}}^{+}\left(k, \frac{1}{4}\right)$, since $\varphi_{0}$ is inside $\mathcal{O}_{\mathbf{m}}^{+}\left(k, \frac{1}{4}\right)$. The estimate (108) holds on the boundary and stable with respect to such a small perturbation of $\varphi$. Thus, estimate (98) is proven.

2. Let $\mathbf{m} \in \mathcal{M},\left|2 k-p_{\mathbf{m}}\right|<1$. Then, $\mathcal{O}_{\mathbf{m}}^{+}$and $\mathcal{O}_{\mathbf{m}}^{-}$can overlap. The case $\varphi_{0} \in$ $\mathcal{O}_{\mathbf{m}}(k, 1) \backslash \mathcal{O}_{\mathbf{m}}(k, 1 / 4)$ we consider in the same way as for $\left|2 k-p_{\mathbf{m}}\right| \geq 1$. Suppose $\varphi_{0} \in \mathcal{O}_{\mathbf{m}}(k, 1 / 4)$. Combining ||$\vec{k}\left(\varphi_{0}\right)+\left.\vec{p}_{\mathbf{m}}\right|_{\mathbb{R}} ^{2}-k^{2} \mid<\frac{1}{4} k^{\delta_{*}}$ with $\left|2 k-p_{\mathbf{m}}\right|<1$, we obtain that the vectors $2 \vec{k}(\varphi)$ and $-\vec{p}_{\mathbf{m}}$ are close:

$$
\left|2 \vec{k}\left(\varphi_{0}\right)+\vec{p}_{\mathbf{m}}\right|_{\mathbb{R}}^{2}<5 k
$$


Therefore, $\left(\vec{k}\left(\varphi_{0}\right)+\vec{p}_{\mathbf{m}}, \vec{p}_{\mathbf{q}}\right)_{\mathbb{R}}=-\left(\vec{k}\left(\varphi_{0}\right), \vec{p}_{\mathbf{q}}\right)_{\mathbb{R}}+O\left(k^{1 / 2+\delta}\right)$ for all $0<\left\|\vec{p}_{\mathbf{q}}\right\|<k^{\delta}$. Considering that the size of $\mathcal{O}_{\mathbf{m}}^{ \pm}$does not exceed $c k^{-1+\delta_{*} / 2}$ (Lemma 3.12) and the distance between $\mathcal{O}_{\mathbf{m}}^{+}$and $\mathcal{O}_{\mathbf{m}}^{-}$is $O\left(k^{-1 / 2}\right)$, we obtain the analogous estimate for all $\varphi$ in $\mathcal{O}_{\mathbf{m}}$ :

$$
\left(\vec{k}(\varphi)+\vec{p}_{\mathbf{m}}, \vec{p}_{\mathbf{q}}\right)_{\mathbb{R}}=-\left(\vec{k}(\varphi), \vec{p}_{\mathbf{q}}\right)_{\mathbb{R}}+O\left(k^{1 / 2+\delta}\right) \quad \text { for all } 0<\left\|\vec{p}_{\mathbf{q}}\right\| \mid<k^{\delta} .
$$

It immediately follows:

$$
\left|\vec{k}(\varphi)+\vec{p}_{\mathbf{m}+\mathbf{q}}\right|_{\mathbb{R}}^{2}-\left|\vec{k}(\varphi)+\vec{p}_{\mathbf{m}}\right|_{\mathbb{R}}^{2}=\left|\vec{k}(\varphi)-\vec{p}_{\mathbf{q}}\right|_{\mathbb{R}}^{2}-|\vec{k}(\varphi)|_{\mathbb{R}}^{2}+O\left(k^{1 / 2+\delta}\right)
$$

for all $0<\left\|\left|\vec{p}_{\mathbf{q}} \|\right|<k^{\delta}\right.$. The size of $\mathcal{O}_{\mathbf{m}}$ is much smaller than that of $\mathcal{O}_{-\mathbf{q}}$ and $\mathcal{O}_{\mathbf{m}}$ is not completely in $\mathcal{O}_{-\mathbf{q}}$. Hence,

$$
|| \vec{k}(\varphi)+\left.\vec{p}_{\mathbf{m}+\mathbf{q}}\right|_{\mathbb{R}} ^{2}-\left|\vec{k}(\varphi)+\vec{p}_{\mathbf{m}}\right|_{\mathbb{R}}^{2} \mid>\frac{1}{2} k^{1-40 \mu \delta}
$$

for all $\varphi \in \mathcal{O}_{\mathbf{m}}$. Considering that ||$\vec{k}(\varphi)+\left.\vec{p}_{\mathbf{m}}\right|_{\mathbb{R}} ^{2}-k^{2} \mid \leq \frac{1}{4} k^{\delta_{*}}$ in $\mathcal{O}_{\mathbf{m}}$, we obtain

$$
|| \vec{k}(\varphi)+\left.\vec{p}_{\mathbf{m}+\mathbf{q}}\right|_{\mathbb{R}} ^{2}-k^{2} \mid>\frac{1}{4} k^{1-40 \mu \delta}, \quad \text { when } \varphi \in \mathcal{O}_{\mathbf{m}} \quad \text { for all } 0<\left\|\left|\vec{p}_{\mathbf{q}} \|\right|<k^{\delta} .\right.
$$

In particular, this means $\mathbf{m} \in \mathcal{M}_{1}\left(\varphi_{0}\right)$, since $\left|\varphi-\varphi_{0}\right|<k^{-2-\delta(40 \mu+1)}$. Considering that ||$\vec{k}(\varphi)+\left.\vec{p}_{\mathbf{m}}\right|_{\mathbb{R}} ^{2}-k^{2} \mid=\frac{1}{4} k^{\delta_{*}}$ on the boundary of $\mathcal{O}_{\mathbf{m}}$, we obtain (104) and (105)). Considering as before, we show that the resolvent (101) has at most two poles inside $\mathcal{O}_{\mathrm{m}}$. It follows that

$$
\left\|\left(P_{\mathbf{m}}\left(H\left(\vec{\varkappa}^{(1)}(\varphi)\right)-k^{2} I\right) P_{\mathbf{m}}\right)^{-1}\right\| \leq k^{-\delta_{*}}\left(c k^{-1+\delta_{*} / 2} / \varepsilon\right)^{2}
$$

when $\varphi$ is on the distance $\varepsilon$ from the pole.

3. Let $\mathbf{m} \in \mathcal{M}, 0<p_{\mathbf{m}} \leq 4 k^{\delta}, \mathbf{m} \notin \Omega(\delta)$. The case $\varphi_{0} \in \mathcal{O}_{\mathbf{m}}(k, 1) \backslash \mathcal{O}_{\mathbf{m}}\left(k, \frac{1}{4}\right)$ is considered the same way as in the previous steps, see (102). From now on we assume $\varphi_{0} \in \mathcal{O}_{\mathbf{m}}\left(k, \frac{1}{4}\right)$. There is an eigenvalue $\lambda^{(1)}\left(\vec{\varkappa}^{(1)}(\varphi)+\vec{p}_{\mathbf{m}}\right)$ of $P_{\mathbf{m}} H\left(\vec{\varkappa}^{(1)}(\varphi)\right) P_{\mathbf{m}}$ given by the perturbation series. Indeed,

$$
|| \vec{k}\left(\varphi_{0}\right)+\left.\vec{p}_{\mathbf{m}}\right|_{\mathbb{R}} ^{2}-k^{2} \mid<\frac{1}{4} k^{\delta_{*}}
$$

since $\varphi_{0} \in \mathcal{O}_{\mathbf{m}}\left(k, \frac{1}{4}\right)$. Considering that $\varphi_{0} \notin \mathcal{O}^{(1)}(k, 8)$, we easily obtain that $\left|\left(\vec{k}\left(\varphi_{0}\right), \vec{p}_{\mathbf{q}}\right)_{\mathbb{R}}\right| \gtrsim k^{1-40 \mu \delta}$ for all $\mathbf{q} \in \Omega(\delta) \backslash\{0\}$. Taking into account that and $p_{\mathbf{m}} \leq 4 k^{\delta}$ we arrive at the estimate:

$$
|| \vec{k}\left(\varphi_{0}\right)+\left.\vec{p}_{\mathbf{m}+\mathbf{q}}\right|_{\mathbb{R}} ^{2}-k^{2} \mid \gtrsim k^{1-40 \mu \delta}
$$


for all $\mathbf{q} \in \Omega(\delta) \backslash\{0\}$ and any $\varphi_{0} \in \omega^{(1)}(k, 8) \cap \mathcal{O}_{\mathbf{m}}\left(k, \frac{1}{4}\right)$. It follows $\mathbf{m} \in \mathcal{M}_{1}$. By Lemma 3.11, $\vec{\varkappa}^{(1)}(\varphi)$ is defined in $\frac{1}{4} k^{-(40 \mu+1) \delta}$-neighborhood of $\omega^{(1)}(k, 8)$, which we denote by $\tilde{\mathcal{W}}^{(1)}\left(k, \frac{1}{4}\right)$. It is easy to show that the estimates similar to the last two hold for $\left.\vec{\varkappa}^{(1)}(\varphi), \varphi \in \tilde{\mathcal{W}}^{(1)}\left(k, \frac{1}{4}\right)\right) \cap \mathcal{O}_{\mathbf{m}}\left(k, \frac{1}{2}\right)$. Therefore,

$$
\begin{gathered}
|| \vec{\varkappa}^{(1)}(\varphi)+\left.\vec{p}_{\mathbf{m}}\right|_{\mathbb{R}} ^{2}-k^{2} \mid \leq \frac{1}{2} k^{\delta_{*}}, \\
|| \vec{\varkappa}^{(1)}(\varphi)+\left.\vec{p}_{\mathbf{m}+\mathbf{q}}\right|^{2}-k^{2} \mid \geq \frac{1}{2} k^{1-40 \mu \delta}
\end{gathered}
$$

for all $\mathbf{q} \in \Omega(\delta) \backslash\{0\}$. It follows from the last two estimates, that the perturbation series for $\lambda^{(1)}\left(\vec{\varkappa}^{(1)}(\varphi)+\vec{p}_{\mathbf{m}}\right)$ and $\lambda^{(1)}\left(\vec{\varkappa}^{(1)}(\varphi)\right)$ converge. Both are holomorphic functions of $\varphi$ in $\tilde{\mathcal{W}}^{(1)}\left(k, \frac{1}{4}\right) \cap \mathcal{O}_{\mathbf{m}}\left(k, \frac{1}{2}\right)$. Using Rouché's Theorem, it is not difficult to show (for details see Appendix 4, Lemma 10.2) that the equation

$$
\lambda^{(1)}\left(\vec{\varkappa}^{(1)}(\varphi)+\vec{p}_{\mathbf{m}}\right)=k^{2}+\varepsilon_{0}, \quad\left|\varepsilon_{0}\right| \leq p_{\mathbf{m}} k^{\delta},
$$

has no more than two solutions $\varphi^{ \pm}\left(\varepsilon_{0}\right)$ in the $\tilde{\mathcal{W}}^{(1)}\left(k, \frac{1}{8}\right) \cap \mathcal{O}_{\mathbf{m}}\left(k, \frac{1}{2}\right)$. They satisfy the estimates:

$$
\left|\varphi^{ \pm}\left(\varepsilon_{0}\right)-\varphi_{\mathbf{m}}^{ \pm}\right|<4 k^{-1+2 \delta} .
$$

Considering that $\varphi_{\mathbf{m}}^{ \pm}=\varphi_{\mathbf{m}} \pm \pi / 2+O\left(k^{-1+\delta}\right)$, we see that the distance between two solutions is approximately equal to $\pi$. For any $\varphi \in \tilde{\mathcal{W}}^{(1)}\left(k, \frac{1}{4}\right) \cap \mathcal{O}_{\mathbf{m}}\left(k, \frac{1}{2}\right)$ satisfying the estimate $\left|\varphi-\varphi_{\mathbf{m}}^{ \pm}\right|<k^{-\delta}$,

$$
\frac{\partial}{\partial \varphi} \lambda^{(1)}\left(\vec{\varkappa}^{(1)}(\varphi)+\vec{p}_{\mathbf{m}}\right)= \pm 2 p_{\mathbf{m}} k(1+o(1)),
$$

for details see Appendix 4, Lemma 10.3. Therefore (for details see Appendix 4, Lemma 10.4),

$$
\left|\lambda^{(1)}\left(\vec{\varkappa}^{(1)}(\varphi)+\vec{p}_{\mathbf{m}}\right)-k^{2}\right| \geq k p_{\mathbf{m}} \varepsilon
$$

if $\varphi \in \tilde{\mathcal{W}}^{(1)}\left(k, \frac{1}{8}\right) \cap \mathcal{O}_{\mathbf{m}}\left(k, \frac{1}{2}\right)$ is outside $\tilde{\mathcal{O}}_{\mathbf{m}, \varepsilon}^{+} \cup \tilde{\mathcal{O}}_{\mathbf{m}, \varepsilon}^{-}$, here and below $\tilde{\mathcal{O}}_{\mathbf{m}, \varepsilon}^{ \pm}$are the open discs of the radius $\varepsilon, 0<\varepsilon<k^{-1+\delta}$, centered at $\varphi^{ \pm}(0)$. It is shown in Appendix 4, Lemma 10.5 that

$$
\left\|\left(\lambda^{(1)}(\vec{y}(\varphi))-k^{2}\right)\left(P_{\mathbf{m}}\left(H\left(\vec{\varkappa}^{(1)}(\varphi)\right)-k^{2}\right) P_{\mathbf{m}}\right)^{-1}\right\| \leq 8, \quad \vec{y}(\varphi):=\vec{\varkappa}^{(1)}(\varphi)+\vec{p}_{\mathbf{m}},
$$

for any $\varphi$ in $\tilde{\mathcal{W}}^{(1)}\left(k, \frac{1}{8}\right) \cap \mathcal{O}_{\mathbf{m}}\left(k, \frac{1}{2}\right)$.

If $\left|\varphi-\varphi_{0}\right|<2 k^{-2-\delta(40 \mu+1)}$ and $\varphi_{0} \in \omega(k, \delta, 8) \cap \mathcal{O}_{\mathbf{m}}\left(k, \frac{1}{4}\right)$, then $\varphi \in \tilde{\mathcal{W}}^{(1)}\left(k, \frac{1}{8}\right) \cap$ $\mathcal{O}_{\mathbf{m}}\left(k, \frac{1}{2}\right)$ and, hence, (115), (116) hold. Now (100) easily follows from (115) and (116). 
Next, we consider the resolvent reduced to a weakly resonant $\mathcal{M}_{2}^{j, s}$.

Lemma 3.23. Let $\mathcal{M}_{2}^{j, s}$ be weakly resonant and let $\left|\varphi-\varphi_{0}\right| \leq \frac{3}{2} k^{-2-40 \mu \delta}$. Then

$$
\left\|\left(H^{j, s}\left(\vec{\varkappa}^{(1)}(\varphi)\right)-k^{2}\right)^{-1}\right\| \leq k^{214 \mu \delta} .
$$

Proof. By (68) and perturbative arguments it is enough to show that

$$
\left\|\left(H^{j, s}(\vec{k}(\varphi))-k^{2}\right)^{-1}\right\|=O\left(k^{213 \mu \delta}\right) .
$$

Let $|\Im \varphi|>k^{-2-128 \mu \delta}$, then (118) follows from (85). Suppose $|\Im \varphi| \leq k^{-2-128 \mu \delta}$. Let $\varphi_{*}=\Re \varphi$. Assume first $\left\|\left(H^{j, s}\left(\vec{k}\left(\varphi_{*}\right)\right)-k^{2}\right)^{-1}\right\|<k^{127 \mu \delta}$. Using perturbative arguments, we obtain $\left\|\left(H^{j, s}(\vec{k}(\varphi))-k^{2}\right)^{-1}\right\|<2 k^{127 \mu \delta}$ and, hence, (118) holds. It remains to assume $\left\|\left(H^{j, s}\left(\vec{k}\left(\varphi_{*}\right)\right)-k^{2}\right)^{-1}\right\| \geq k^{127 \mu \delta}$. Considering ( $(\underline{86})$, we obtain that there is a real pole $\varphi_{* *}$ of the resolvent in the $k^{-2-42 \mu \delta}$-neighborhood of $\varphi_{*}$. It follows that $\varphi$ is in the $k^{-2-41 \mu \delta_{-}}$ neighborhood of a pole $\varphi_{* *}$ of the resolvent. This contradicts to the definition of the weakly resonant $\mathcal{M}_{2}^{j, s}$. Thus, (118) and, hence, (117) are proven.

Now, we discuss the part of the resolvent corresponding to strongly resonant blocks. Let us consider a trivial $\mathcal{M}_{2}^{j}$ and a cluster $\mathcal{M}_{2, s t r}^{j, s}$ of strongly resonant sets, see Definition 3.19 and the text after. We denote by $\tilde{\mathcal{M}}_{2, s t r}^{j, s}$ the $\frac{1}{3} k^{\delta}$-neighborhood (in $\||\cdot \||$-norm) of $\mathcal{M}_{2, s t r}^{j, s}$. By definition of a cluster, such neighborhoods of different clusters are disjoint.

Let $P_{s t r}$ be the projection corresponding to a $\tilde{\mathcal{M}}_{2, s t r}^{j, s}$. We consider the operator $P_{s t r} H\left(\vec{\varkappa}^{(1)}(\varphi)\right) P_{s t r}$. By $d_{s t r}(\mathbf{n})$ we denote the $\||\cdot \||$-distance from a point $\mathbf{n}$ to the nearest strongly resonant $\mathcal{M}_{2}^{j, s}$, i.e.,

$$
d_{s t r}(\mathbf{n}):=\min _{\mathbf{m}}\left\|\left|\vec{p}_{\mathbf{n}-\mathbf{m}} \|\right|,\right.
$$

where the minimum is over all $\mathbf{m}$ in strongly resonant $\mathcal{M}_{2}^{j, s}$.

Lemma 3.24. Let $\mathcal{M}_{2}^{j}$ be trivial. Then, the resolvent $\left(P_{\text {str }}\left(H\left(\vec{\varkappa}^{(1)}(\varphi)\right)-k^{2}\right) P_{\text {str }}\right)^{-1}$ has at most 4 poles in the disc $\left|\varphi-\varphi_{0}\right| \leq k^{-2-40 \mu \delta}$. The resolvent obeys the estimate

$$
\left\|\left(P_{s t r}\left(H\left(\vec{\varkappa}^{(1)}(\varphi)\right)-k^{2}\right) P_{s t r}\right)^{-1}\right\| \leq k^{127 \mu \delta}\left(\frac{k^{-2-41 \mu \delta}}{\varepsilon_{0}}\right)^{4},
$$

where $\varepsilon_{0}=\min \left\{\varepsilon, k^{-2-41 \mu \delta}\right\}$ with $\varepsilon$ being the distance from $\varphi$ to the nearest pole of the resolvent, $\vec{\varkappa}^{(1)}(\varphi)$ being defined in Section 3.4.

Moreover, the following estimate for the matrix elements holds:

$$
\begin{aligned}
& \left|\left(P_{s t r}\left(H\left(\vec{\varkappa}^{(1)}(\varphi)\right)-k^{2}\right) P_{s t r}\right)_{\mathbf{m m}^{\prime}}^{-1}-\left(P_{s t r}\left(H_{0}\left(\vec{\varkappa}^{(1)}(\varphi)\right)-k^{2}\right) P_{s t r}\right)_{\mathbf{m m}^{\prime}}^{-1}\right| \leq \\
& \left(\frac{k^{-2-41 \mu \delta}}{\varepsilon_{0}}\right)^{4} k^{-\delta_{*} d\left(\mathbf{m}, \mathbf{m}^{\prime}\right)}+k^{-\delta_{*} / 9},
\end{aligned}
$$


where

$$
d\left(\mathbf{m}, \mathbf{m}^{\prime}\right)=\frac{d_{s t r}(\mathbf{m})+d_{s t r}\left(\mathbf{m}^{\prime}\right)}{20 Q}, \quad d_{s t r}(\mathbf{m})+d_{s t r}\left(\mathbf{m}^{\prime}\right)>20 Q
$$

Proof. First we consider the resolvent of the free operator: $\left(P_{s t r}\left(H_{0}(\vec{k}(\varphi))-k^{2}\right) P_{s t r}\right)^{-1}$. Its poles are zeros of the function (92) for $\mathbf{m}$ being in the $\frac{1}{3} k^{\delta}$-neighborhood of a strongly resonant cluster (in particular, either $\mathbf{m} \in \mathcal{M}_{2}^{j}$ or $\mathbf{m} \notin \mathcal{M}\left(\varphi_{0}\right)$ ). It follows from Lemma 3.21 that (92) does not have zeros in $\left|\varphi-\varphi_{0}\right| \leq k^{-2-40 \mu \delta}$ for $\mathbf{m} \notin \mathcal{N}_{2}^{j}$. Let $\mathbf{m} \in \mathcal{M}_{2}^{j}$. Then, (92) has no more than two zeros. Using Lemma 3.20, we obtain that the resolvent of the free operator has no more than four poles.

Let $\tilde{\mathcal{O}}$ be the union of the discs of radius $k^{-2-41 \mu \delta}$ around poles of $\left(P_{s t r}\left(H_{0}(\vec{k}(\varphi))-\right.\right.$ $\left.\left.k^{2}\right) P_{s t r}\right)^{-1}$ in the $2 k^{-2-40 \mu \delta}$-neighborhood of $\varphi_{0}$. Obviously, there is a circle $\left|\varphi-\varphi_{0}\right|=$ $c k^{-2-40 \mu \delta}$ with $1 \leq c \leq \frac{3}{2}$ which does not intersect $\tilde{\mathcal{O}}$. Further we reduce our considerations to the disc bounded by this circle, since it contains $\left|\varphi-\varphi_{0}\right|<k^{-2-40 \mu \delta}$ and any connected component of $\tilde{\mathcal{O}}$ which intersect this disc is strongly inside it.

We show that in the disc $\left|\varphi-\varphi_{0}\right| \leq c k^{-2-40 \mu \delta}$ the resolvent of the perturbed operator $\left(P_{s t r}\left(H(\vec{k}(\varphi))-k^{2}\right) P_{s t r}\right)^{-1}$ has no poles outside $\tilde{\mathcal{O}}$ and it has the same number of poles in $\tilde{\mathcal{O}}$ as the resolvent of the free operator. Indeed, let us consider the perturbation series for the resolvent:

$$
\begin{aligned}
& \left(P_{s t r}\left(H(\vec{k}(\varphi))-k^{2}\right) P_{s t r}\right)^{-1}= \\
& \sum_{r=0}^{\infty}\left(P_{s t r}\left(H_{0}(\vec{k}(\varphi))-k^{2}\right) P_{s t r}\right)^{-1}\left(-V\left(P_{s t r}\left(H_{0}(\vec{k}(\varphi))-k^{2}\right) P_{s t r}\right)^{-1}\right)^{r} .
\end{aligned}
$$

The series converges if $\|V A\|<1$, where

$$
A(\vec{k}(\varphi)):=\left(P_{s t r}\left(H_{0}(\vec{k}(\varphi))-k^{2}\right) P_{s t r}\right)^{-1} V\left(P_{s t r}\left(H_{0}(\vec{k}(\varphi))-k^{2}\right) P_{s t r}\right)^{-1}
$$

We prove that

$$
\|A(\vec{k}(\varphi))\| \leq k^{132 \mu \delta-\delta_{*} / 2}<k^{-\delta_{*} / 3},
$$

when $\varphi \notin \tilde{\mathcal{O}}$. First, using (87), we easily obtain that

$$
|| \vec{k}(\varphi)+\left.\vec{p}_{\mathbf{m}}\right|^{2}-k^{2} \mid \geq k^{-126 \mu \delta} \quad \text { when } \mathbf{m} \in \mathcal{M}_{2}^{j}, \varphi \notin \tilde{\mathcal{O}},\left|\varphi-\varphi_{0}\right|<c k^{-2-40 \mu \delta} .
$$

Combining (97) and (125), we obtain:

$$
\left\|\left(P_{s t r}\left(H_{0}(\vec{k}(\varphi))-k^{2}\right) P_{s t r}\right)^{-1}\right\| \leq k^{126 \mu \delta} \text { when } \varphi \notin \tilde{\mathcal{O}},\left|\varphi-\varphi_{0}\right|<c k^{-2-40 \mu \delta} .
$$

Next, let $\mathcal{M}_{2}^{j}$ be such that corresponding direction $\mathbf{q}$ does not belong to $\mathcal{S}_{Q}$ (the first option from the definition of a trivial $\mathcal{M}_{2}^{j}$ ). Assume $\mathbf{m}, \mathbf{m}^{\prime} \in \mathcal{M}_{2}^{j}$. Note that $A_{\mathbf{m m}^{\prime}}$ can differ from zero only if $\mathbf{m}-\mathbf{m}^{\prime} \in \mathcal{S}_{Q} \backslash\{\mathbf{0}\}$. On the other hand $\mathbf{m}-\mathbf{m}^{\prime}=w \mathbf{q}$, where $\mathbf{q}$ 
is the directional vector of $\mathcal{M}_{2}^{j}, w \in \mathbb{R}$. This contradicts to assumption $\mathbf{q} \notin \mathcal{S}_{Q}$. Thus, if $A_{\mathbf{m m}^{\prime}} \neq 0$ we have either $\mathbf{m} \notin \mathcal{M}_{2}^{j}$ or $\mathbf{m}^{\prime} \notin \mathcal{M}_{2}^{j}$. Using (97), we get

$$
\left|A_{\mathbf{m m}^{\prime}}\right| \leq 2 k^{-\delta_{*}}\|V\| k^{126 \mu \delta} .
$$

It is easy to see that the number of $\mathbf{m}$ in a cluster of boxes does not exceed $c k^{4 \delta}$, Therefore, (124) holds. Now, let $\mathbf{q} \in \mathcal{S}_{Q}$, but

$$
\left|k^{2}-\left(\vec{k}+\vec{p}_{\mathbf{m}}, \vec{\nu}_{\mathbf{q}}^{\perp}\right)^{2}\right| \geq k^{\delta_{*}} / 8
$$

when $\mathbf{m} \in \mathcal{M}_{2}^{j}$ (the second option from Definition 3.15 of a trivial $\mathcal{M}_{2}^{j}$ ). Let us show that

$$
|| \vec{k}+\left.\vec{p}_{\mathbf{m}}\right|_{\mathbb{R}} ^{2}-k^{2}|+|\left|\vec{k}+\vec{p}_{\mathbf{m}^{\prime}}\right|_{\mathbb{R}}^{2}-k^{2} \mid>k^{\delta_{*} / 2-\delta} .
$$

Suppose the inequality does not hold. As above, if $A_{\mathbf{m m}^{\prime}} \neq 0$ then $\mathbf{m}-\mathbf{m}^{\prime}=n_{0} \mathbf{q}$, $n_{0} \in \mathbb{Z} \backslash\{0\}$ and $\left|n_{0}\right| \|\left|\vec{p}_{\mathbf{q}}\right||| \leq Q$. It follows:

$$
\left|2\left(\vec{k}+\vec{p}_{\mathbf{m}}, n_{0} p_{\mathbf{q}}\right)+\left(n_{0} \vec{p}_{\mathbf{q}}\right)^{2}\right| \leq 2 k^{\delta_{*} / 2-\delta} .
$$

Considering (127) and the inequality opposite to (128), we obtain

$$
\left|\left(\vec{k}+\vec{p}_{\mathbf{m}}, \vec{\nu}_{\mathbf{q}}\right)\right|^{2}>\frac{1}{16} k^{\delta_{*}}
$$

which contradicts (129). Hence, (128) holds when $A_{\mathbf{m m}^{\prime}} \neq 0$. Using (125) and the inequality (128), we obtain (124) for the second case of a trivial $\mathcal{M}_{2}^{j}$.

Estimates (124) and (126) yield that the series (122) converges and

$$
\left\|\left(P_{s t r}\left(H(\vec{k}(\varphi))-k^{2}\right) P_{s t r}\right)^{-1}\right\|<2 k^{126 \mu \delta}, \text { when } \varphi \notin \tilde{\mathcal{O}},\left|\varphi-\varphi_{0}\right|<c k^{-2-40 \mu \delta} .
$$

Next, we show that $\left(P_{s t r}\left(H_{0}(\vec{k}(\varphi))-k^{2}\right) P_{s t r}\right)^{-1}$ and $\left(P_{s t r}\left(H(\vec{k}(\varphi))-k^{2}\right) P_{s t r}\right)^{-1}$ have the same number of poles (counting algebraic multiplicity) inside $\tilde{\mathcal{O}}$. Indeed, we introduce $H_{\beta}=P_{\text {str }}\left(H_{0}+\beta V\right) P_{\text {str }}, 0 \leq \beta \leq 1$. The series for the resolvent converges on the boundary of $\tilde{\mathcal{O}}$ uniformly in $\beta$. Thus, the determinant $D_{\beta}:=\operatorname{det}\left(H_{\beta}(\vec{k}(\varphi))-k^{2} I\right)$ is the polynomial in $\beta$ uniformly bounded from below on the boundary of $\tilde{\mathcal{O}}$. Now, it follows from continuity that in each connected component of $\tilde{\mathcal{O}}$ the determinant $D_{\beta}$ has the same number of zeros for all $0 \leq \beta \leq 1$. Thus, $\left(P_{s t r}\left(H(\vec{k}(\varphi))-k^{2}\right) P_{s t r}\right)^{-1}$ has the same number of poles in $\tilde{\mathcal{O}}$ as the unperturbed operator $\left(P_{s t r}\left(H_{0}(\vec{k}(\varphi))-k^{2}\right) P_{s t r}\right)^{-1}$, i.e. not more than 4. We note that in what follows we will often use similar arguments without additional comments.

Now, we can apply (68) and pertubative arguments to (124) - (130) to obtain

$$
\| A\left(\left(\overrightarrow{\mathcal{\varkappa}}^{(1)}(\varphi)\right) \|<k^{-\delta_{*} / 4} \text {, when } \varphi \notin \tilde{\mathcal{O}},\left|\varphi-\varphi_{0}\right|<c k^{-2-40 \mu \delta},\right.
$$




$$
\begin{gathered}
\left\|\left(P_{s t r}\left(H_{0}\left(\vec{\varkappa}^{(1)}(\varphi)\right)-k^{2}\right) P_{s t r}\right)^{-1}\right\| \leq 2 k^{126 \mu \delta}, \text { when } \varphi \notin \tilde{\mathcal{O}},\left|\varphi-\varphi_{0}\right|<c k^{-2-40 \mu \delta}, \\
\left\|\left(P_{s t r}\left(H\left(\vec{\varkappa}^{(1)}(\varphi)\right)-k^{2}\right) P_{s t r}\right)^{-1}\right\|<4 k^{126 \mu \delta}, \text { when } \varphi \notin \tilde{\mathcal{O}},\left|\varphi-\varphi_{0}\right|<c k^{-2-40 \mu \delta} .
\end{gathered}
$$

To show that $\left(P_{s t r}\left(H\left(\vec{\varkappa}^{(1)}(\varphi)\right)-k^{2}\right) P_{s t r}\right)^{-1}$ and $\left(P_{s t r}\left(H(\vec{k}(\varphi))-k^{2}\right) P_{s t r}\right)^{-1}$ have the same number of poles (counting algebraic multiplicity) inside $\tilde{\mathcal{O}}$ we apply the arguments as above for the determinant $D_{t}:=\operatorname{det}\left(H\left((1-t) \vec{k}(\varphi)+t \vec{\varkappa}^{(1)}(\varphi)\right)-k^{2} I\right), 0 \leq t \leq 1$. Finally, using the maximum principle in $\mathcal{O}$ and (133), we get (119)).

Next, we prove (120). We rewrite a matrix element of $\left(P_{s t r}\left(H\left(\vec{\varkappa}^{(1)}(\varphi)\right)-k^{2}\right) P_{s t r}\right)^{-1}$ in the form

$$
\begin{aligned}
& \left(P_{s t r}\left(H\left(\vec{\varkappa}^{(1)}(\varphi)\right)-k^{2}\right) P_{s t r}\right)_{\mathbf{m m}^{\prime}}^{-1}= \\
& \sum_{r=0}^{R}\left(\left(P_{s t r}\left(H_{0}\left(\vec{\varkappa}^{(1)}(\varphi)\right)-k^{2}\right) P_{s t r}\right)^{-1}\left(-V\left(P_{s t r}\left(H_{0}\left(\vec{\varkappa}^{(1)}(\varphi)\right)-k^{2}\right) P_{s t r}\right)^{-1}\right)^{r}\right)_{\mathbf{m m}^{\prime}}+ \\
& \left(\left(P_{s t r}\left(H\left(\vec{\varkappa}^{(1)}(\varphi)\right)-k^{2}\right) P_{s t r}\right)^{-1}\left(-V\left(P_{s t r}\left(H_{0}\left(\vec{\varkappa}^{(1)}(\varphi)\right)-k^{2}\right) P_{s t r}\right)^{-1}\right)^{R+1}\right)_{\mathbf{m m}^{\prime}},
\end{aligned}
$$

where we choose $R:=\left[\frac{d_{s t r}\left(\mathbf{m}^{\prime}\right)}{Q}\right]-2$ (here, without the loss of generality we assume $\left.d_{s t r}\left(\mathbf{m}^{\prime}\right) \geq d_{s t r}(\mathbf{m})\right)$. Note that $\mathbf{m}^{\prime} \notin \mathcal{M}_{2}^{j, s}$ (strongly resonant) by (121). Let us consider the sum in the right-hand side. By induction, one can easily see that the sum in the right-hand side part is holomorphic in $\tilde{\mathcal{O}}\left(R:=\left[\frac{d_{s t r}\left(\mathbf{m}^{\prime}\right)}{Q}\right]-2\right)$, since (32) is valid. If $\varphi \notin \tilde{\mathcal{O}}$, then we use (132) for $\mathbf{m}, \mathbf{m}^{\prime}$ and (131) to show:

$$
\begin{array}{r}
\left|\left(\left(P_{s t r}\left(H_{0}\left(\vec{\varkappa}^{(1)}(\varphi)\right)-k^{2}\right) P_{s t r}\right)^{-1}\left(V\left(P_{s t r}\left(H_{0}\left(\vec{\varkappa}^{(1)}(\varphi)\right)-k^{2}\right) P_{s t r}\right)^{-1}\right)^{r}\right){ }_{\mathbf{m m}^{\prime}}\right| \\
2 k^{126 \mu \delta-\delta_{*} r / 8}, \quad r \geq 1 .
\end{array}
$$

Since the sum is holomorphic in $\tilde{\mathcal{O}}$ it can be estimated by the maximum principle inside $\tilde{\mathcal{O}}$. Using (119), (132) and (131), we obtain that the last term in the right-hand side of (134) is bounded by $\left(\frac{k^{-2-41 \mu \delta}}{\varepsilon_{0}}\right)^{4} k^{-\delta_{*} d\left(\mathbf{m}, \mathbf{m}^{\prime}\right)}$. Now (120) easily follows.

We also notice that from (132) and the maximum principle one has the estimate (cf. (135), (117))

$$
\left|\left(P_{s t r}\left(H_{0}\left(\vec{\varkappa}^{(1)}(\varphi)\right)-k^{2}\right) P_{s t r}\right)_{\mathbf{m m}}^{-1}\right| \leq 2 k^{126 \mu \delta}, \quad \text { when } d_{s t r}(\mathbf{m})>0,
$$

uniformly in the disc $\left|\varphi-\varphi_{0}\right| \leq k^{-2-40 \mu \delta}$.

Now, we consider the case of a non-trivial $\mathcal{M}_{2}^{j}$. Let $\mathcal{M}_{2, s t r}^{j, s}$ be a strongly resonant cluster, see Definition 3.19 and the text after. We need to consider its $k^{\delta}$-neighborhood in $\||\cdot| \mid-$-norm. According to Lemma 3.20 and the definition of a cluster, such a neighborhood contains no more than two strongly resonant sets $\mathcal{M}_{2}^{j, s}$. A slight technical complication is that such a neighborhood can intersect weak clusters. If a weak cluster is 
completely inside the neighborhood, it is not a problem. If a weak cluster sticks out of the neighborhood we have to "attach" it to the neighborhood as a whole. Here are more technical details. Let $\mathcal{M}_{2, s t r}^{j, s}$ be a strongly resonant cluster. We consider its $\frac{1}{6} C(Q)^{-1} k^{\delta}$ neighborhood, where $C(Q)$ is as in Appendix 3 (without the loss of generality, $C(Q) \geq 1$ ). We also consider a slightly bigger $\frac{1}{5} C(Q)^{-1} k^{\delta}$-neighborhood of $\mathcal{M}_{2, s t r}^{j, s}$. If a weakly resonant $\mathcal{M}_{2}^{j, s^{\prime \prime}}$ intersects the bigger neighborhood, then we attach the whole $\mathcal{M}_{2}^{j, s^{\prime \prime}}$ to the smaller $\frac{1}{6} C(Q)^{-1} k^{\delta}$-neighborhood of $\mathcal{M}_{2, s t r}^{j, s}$. We call this object the extended $\frac{1}{6} C(Q)^{-1} k^{\delta}$ neighborhood of $\mathcal{M}_{2, s t r}^{j, s}$ and denote by $\tilde{\mathcal{M}}_{2, s t r}^{j, s}$. In other words, our extended neighborhood contains the "body", which is $\frac{1}{6} C(Q)^{-1} k^{\delta}$-neighborhood of $\mathcal{M}_{2, s t r}^{j, s}$ and the branches, which are all weakly resonant $\mathcal{M}_{2}^{j, s^{\prime \prime}}$ intersecting the bigger $\frac{1}{5} C(Q)^{-1} k^{\delta}$-neighborhood of $\mathcal{M}_{2, s t r}^{j, s}$. Thus, this branches can be, in fact, disjoint from the body. At the same time, such definition will be very convenient later as (by construction of $\mathcal{M}_{2}^{j, s^{\prime \prime}}$ ) our extended neighborhood is not connected by potential $V$ with any new weakly resonant $\mathcal{M}_{2}^{j, s^{\prime \prime}}$ (see below the construction of the model operator). Note that any weak "branch" $\mathcal{M}_{2}^{j, s "}$ considered above can be included into the $\frac{1}{3} k^{\delta}$-neighborhood of $\mathcal{M}_{2, s t r}^{j, s}$, see Appendix 3. This means that the extended neighborhood $\tilde{\mathcal{M}}_{2, s t r}^{j, s}$ belongs to the $\frac{1}{3} k^{\delta}$-neighborhood of $\mathcal{M}_{2, s t r}^{j, s}$, but, generally speaking, does not coincide with it. Note that extended neighborhoods of any two different clusters $\mathcal{M}_{2, s t r}^{j, s}$ are disjoint. It follows from the definition of a cluster and the fact that each extended neighborhood belongs to the $\frac{1}{3} k^{\delta}$-neighborhood of $\mathcal{M}_{2, s t r}^{j, s}$.

By definition, $\mathcal{M}_{2, s t r}^{j, s}=\cup_{s} \mathcal{M}_{2}^{j, s}$, the union is taken over all $\mathcal{M}_{2}^{j, s}$ belonging to the cluster. Let $P_{j}$ be the projection corresponding to this union, while $P_{j, s}$ are the projections corresponding to sets $\mathcal{M}_{2}^{j, s}$. We put $H^{j, s}:=P_{j, s} H P_{j, s}, \tilde{H}^{j}=\oplus_{s} H^{j, s}$ and $\hat{H}:=\tilde{H}^{j}+$ $H_{0}\left(P_{s t r}-P_{j}\right)$, where $P_{s t r}$ is the projection corresponding to $\tilde{\mathcal{M}}_{2, s t r}^{j, s}$. Hence,

$$
P_{s t r} H P_{s t r}=\hat{H}+W, \quad W:=P_{s t r} V P_{s t r}-\sum_{s} P_{j, s} V P_{j, s}
$$

Lemma 3.25. Let $\mathcal{M}_{2}^{j}$ be non-trivial. The resolvent $\left(P_{\text {str }}\left(H\left(\vec{\varkappa}^{(1)}(\varphi)\right)-k^{2}\right) P_{\text {str }}\right)^{-1}$ has at most 4 poles in the disc $\left|\varphi-\varphi_{0}\right| \leq k^{-2-40 \mu \delta}$. It obeys the estimate

$$
\left\|\left(P_{s t r}\left(H\left(\vec{\varkappa}^{(1)}(\varphi)\right)-k^{2}\right) P_{s t r}\right)^{-1}\right\| \leq k^{215 \mu \delta}\left(\frac{k^{-2-41 \mu \delta}}{\varepsilon_{0}}\right)^{4},
$$

where $\varepsilon_{0}=\min \left\{\varepsilon, k^{-2-41 \mu \delta}\right\}$ with $\varepsilon$ being the distance from $\varphi$ to the nearest pole of the resolvent.

Moreover, the following estimate for the matrix elements holds:

$$
\begin{aligned}
& \left|\left(P_{s t r}\left(H\left(\vec{\varkappa}^{(1)}(\varphi)\right)-k^{2}\right) P_{s t r}\right)_{\mathbf{m m}^{\prime}}^{-1}-\left(P_{s t r}\left(\hat{H}\left(\vec{\varkappa}^{(1)}(\varphi)\right)-k^{2}\right) P_{s t r}\right)_{\mathbf{m m}^{\prime}}^{-1}\right| \leq \\
& \left(\frac{k^{-2-41 \mu \delta}}{\varepsilon_{0}}\right)^{4} k^{-\delta_{*} d\left(\mathbf{m}, \mathbf{m}^{\prime}\right)}+k^{-\delta_{*} / 9}
\end{aligned}
$$


where

$$
d\left(\mathbf{m}, \mathbf{m}^{\prime}\right)=\frac{d_{s t r}(\mathbf{m})+d_{s t r}\left(\mathbf{m}^{\prime}\right)}{20 C(Q) Q}, \quad d_{s t r}(\mathbf{m}), d_{s t r}\left(\mathbf{m}^{\prime}\right)>10 C(Q) Q .
$$

Here $C(Q)$ is the constant from Appendix 3.

Proof. Let $A:=\left(\hat{H}-k^{2}\right)^{-1} W\left(\hat{H}-k^{2}\right)^{-1}$ and $\tilde{\mathcal{O}}$ be the $k^{-2-41 \mu \delta}$-neighborhood of the poles of $\left(\hat{H}(\vec{k}(\varphi))-k^{2}\right)^{-1}$ in the $2 k^{-2-40 \mu \delta}$-neighborhood of $\varphi_{0}$. Obviously, such poles are just poles of $\left(H^{j, s}(\vec{k}(\varphi))-k^{2}\right)^{-1}$ corresponding to strongly resonant $\mathcal{M}_{2}^{j, s}$. In particular, we have no more than 4 poles and (cf. above) there exists $c \in[1,3 / 2]$ such that the circle $\left|\varphi-\varphi_{0}\right|=c k^{-2-40 \mu \delta}$ does not intersect $\tilde{\mathcal{O}}$. Considering as in the proof of Lemma 3.23 we obtain the estimate

$$
\left\|\left(\hat{H}(\vec{k}(\varphi))-k^{2}\right)^{-1}\right\|=O\left(k^{213 \mu \delta}\right) \text {, when }\left|\varphi-\varphi_{0}\right| \leq c k^{-2-40 \mu \delta} \text { and } \varphi \notin \tilde{\mathcal{O}} \text {. }
$$

We just note that condition $\varphi \notin \tilde{\mathcal{O}}$ ensures that the proof holds in the case of a strongly resonant $\mathcal{M}_{2}^{j, s}$.

If $\mathbf{m}^{\prime} \notin \cup_{s} \mathcal{M}_{2}^{j, s}$ then

$$
\left|\left(\left(\hat{H}-k^{2}\right)^{-1}\right)_{\mathbf{m m}^{\prime}}\right|=\left|\delta_{\mathbf{m m}^{\prime}}\left(\left(H_{0}-k^{2}\right)^{-1}\right) \mathbf{m m}^{\prime}\right| \leq k^{-\delta_{*}} .
$$

Thus,

$$
\left\|A\left(P_{s t r}-P_{j}\right)\right\| \leq\|V\| k^{214 \mu \delta-\delta_{*}} \leq k^{-\delta_{*} / 2}
$$

Next,

$$
P_{j} A P_{j}=\sum_{s, s^{\prime}}\left(H^{j, s}-k^{2}\right)^{-1} P_{j, s} W P_{j, s^{\prime}}\left(H^{j, s^{\prime}}-k^{2}\right)^{-1}
$$

Let us show that $P_{j} A P_{j}=0$. Indeed,

$$
P_{j, s} W P_{j, s^{\prime}}=P_{j, s} V P_{j, s^{\prime}}-P_{j, s}\left(\sum_{s^{\prime \prime}} P_{j, s^{\prime \prime}} V P_{j, s^{\prime \prime}}\right) P_{j, s^{\prime}}
$$

Let us show that the last expression is equal to zero. It is obvious for $s=s^{\prime}$. If $s \neq s^{\prime}$ we have $P_{j, s} W P_{j, s^{\prime}}=P_{j, s} V P_{j, s^{\prime}}$. Consider $V_{\mathbf{m m}^{\prime}}$ with $\mathbf{m} \in \mathcal{M}_{2}^{j, s}, \mathbf{m}^{\prime} \in \mathcal{M}_{2}^{j, s^{\prime}}$. We have $\mathbf{m}-\mathbf{m}^{\prime}=w \mathbf{q}$. If $V_{\mathbf{m m}^{\prime}} \neq 0$, then $\mathbf{m}-\mathbf{m}^{\prime} \in \mathcal{S}_{Q}$ and hence, by the second condition on the potential, $w$ is integer. But this means $\mathcal{M}_{2}^{j, s}=\mathcal{M}_{2}^{j, s^{\prime}}$. Hence, $P_{j, s} W P_{j, s^{\prime}}=0$ for every $s, s^{\prime}$. Therefore, $P_{j} A P_{j}=0$. Combining this with (141), we obtain the estimate analogous to (124). Further, applying arguments from the proof of Lemma 3.24 and (140), we obtain (137). 
It remains to estimate the matrix elements. We have

$$
\begin{aligned}
& \left(P_{\text {str }}\left(H\left(\vec{\varkappa}^{(1)}(\varphi)\right)-k^{2}\right) P_{\text {str }}\right)_{\mathbf{m m}^{\prime}}^{-1}= \\
& \sum_{r=0}^{R}\left(\left(\hat{H}\left(\vec{\varkappa}^{(1)}(\varphi)\right)-k^{2}\right)^{-1}\left(-W\left(\hat{H}\left(\vec{\varkappa}^{(1)}(\varphi)\right)-k^{2}\right)^{-1}\right)^{r}\right)_{\mathbf{m m}^{\prime}}+ \\
& \left(\left(P_{\text {str }}\left(H\left(\vec{\varkappa}^{(1)}(\varphi)\right)-k^{2}\right) P_{\text {str }}\right)^{-1}\left(-W\left(\hat{H}\left(\vec{\varkappa}^{(1)}(\varphi)\right)-k^{2}\right)^{-1}\right)^{R+1}\right)_{\mathbf{m m}^{\prime}},
\end{aligned}
$$

where $R:=\left[\frac{d\left(\mathbf{m}^{\prime}\right)}{C(Q) Q}\right]-2$. Here, as before, without the loss of generality we assume that $d\left(\mathbf{m}^{\prime}\right) \geq d(\mathbf{m})$. Let us show that the sum in the right hand side part is holomorphic in $\tilde{\mathcal{O}}$. It is easy to see that this sum is a combination (including products and sums) of the operators of the type

$$
\left(H^{j, s_{1}}-k^{2}\right)^{-1}\left(W\left(P_{s t r}-P_{j}\right)\left(H_{0}-k^{2}\right)^{-1}\right)^{t} W\left(H^{j, s_{2}}-k^{2}\right)^{-1}, t \geq 0,
$$

where $\left(P_{s t r}-P_{j}\right)\left(H_{0}-k^{2}\right)^{-1}$ is just a diagonal part of $\left.\hat{H}\left(\vec{\varkappa}^{(1)}(\varphi)\right)-k^{2}\right)^{-1}$. Operators of the type (143) without the end terms also can be present. Since $W_{\mathbf{q q}^{\prime}}=0$ when $\left\|\left|\vec{p}_{\mathbf{q}-\mathbf{q}^{\prime}} \|\right|>Q\right.$, the operator (143) can differ from zero only when the $\left\||\cdot \||\right.$-distance between $\mathcal{M}_{2}^{j, s_{1}}$ and $\mathcal{M}_{2}^{j, s_{2}}$ is less than $(t+1) Q$. Therefore (see Appendix 3), the $\||\cdot \||$-distance between their central points is less than $C(Q)(t+1) Q$. Consequently, if any term in the finite sum of (142) includes a strongly resonant block of $\hat{H}$, this term is equal to zero unless the $\||\cdot \||-$ distance between the central point of the strongly resonant $\mathcal{M}_{2}^{j, s}$ and the central point of a weakly resonant block containing $\mathbf{m}^{\prime}$ is less than $R C(Q) Q$, if $\mathbf{m}^{\prime}$ is in a resonant block. By Appendix 3, $\||\cdot|||$-distance between $\mathbf{m}^{\prime}$ and the strongly resonant $\mathcal{M}_{2}^{j, s}$ is not greater than $R C(Q) Q+\left\|\left|\vec{p}_{\mathbf{q}} \|\right| \leq(R+1) C(Q) Q\right.$, otherwise the corresponding term is just zero. This contradicts to the definition of $R, R:=\left[\frac{d\left(\mathbf{m}^{\prime}\right)}{C(Q) Q}\right]-2$. If $\mathbf{m}^{\prime}$ is not in a resonant block, then the considerations are, obviously, similar, just simpler.

Now, the proof of (138) can be completed in the same way as in the proof of Lemma 3.24

We also notice that from (140), (68) and the maximum principle one has the estimate (cf. (117))

$$
\left|\left(P_{s t r}\left(\hat{H}\left(\vec{\varkappa}^{(1)}(\varphi)\right)-k^{2}\right) P_{s t r}\right)_{\mathbf{m m}^{\prime}}^{-1}\right| \leq k^{214 \mu \delta}, \text { when } d_{s t r}(\mathbf{m})+d_{s t r}\left(\mathbf{m}^{\prime}\right)>0,
$$

uniformly in the disc $\left|\varphi-\varphi_{0}\right| \leq k^{-2-40 \mu \delta}$.

Now, we put

$$
P=\sum_{\mathbf{m} \in \mathcal{M}_{1}} P_{\mathbf{m}}+\sum_{j, \text { trivial }} \sum_{t} P_{2, \text { str }}^{j, t}+\sum_{j, \text { non-trivial }}\left(\sum_{s} P_{2, w e a k}^{j, s}+\sum_{t} P_{2, \text { str }}^{j, t}\right)
$$

where $P_{\mathbf{m}}$ are diagonal projectors corresponding to the sets $\tilde{\mathcal{M}}_{\mathbf{m}}, P_{2, \text { weak }}^{j, s}$ correspond to weakly resonant $\mathcal{M}_{2}^{j, s}$ and $P_{2, s t r}^{j, t}$ correspond to all different $\tilde{\mathcal{M}}_{2, s t r}^{j, t}$ introduced before Lemmas 3.24 and 3.25. In (145) we take the sum over all (non-trivial) weakly resonant sets, 
which are disjoint from any $\tilde{\mathcal{M}}_{2, s t r}^{j, t}$ and over all $\mathbf{m} \in \mathcal{M}_{1}$, which are not in any $\tilde{\mathcal{M}}_{2, s t r}^{j, t}$ or a weakly resonant set of a non-trivial $\mathcal{M}_{2}^{j}$.

Lemma 3.26. The projectors in (145) are mutually orthogonal:

$$
\begin{gathered}
P_{\mathbf{m}} V P_{\mathbf{m}^{\prime}}=P_{\mathbf{m}} V P_{2, \text { weak }}^{j, s}=P_{\mathbf{m}} V P_{2, \text { str }}^{j, t}=0 \text {, when } \mathbf{m}, \mathbf{m}^{\prime} \in \mathcal{M}_{1}, \mathbf{m} \neq \mathbf{m}^{\prime}, \\
P_{2, \text { str }}^{j, t} V P_{2, \text { str }}^{j^{\prime}, t^{\prime}}=0 \text {, when }(j, t) \neq\left(j^{\prime}, t^{\prime}\right),
\end{gathered}
$$

where $\mathcal{M}_{2}^{j}$ and $\mathcal{M}_{2}^{j^{\prime}}$ are trivial or non-trivial; and

$$
P_{2, \text { weak }}^{j, s} V P_{2, \text { weak }}^{j^{\prime}, s^{\prime}}=0, \text { when }(j, s) \neq\left(j^{\prime}, s^{\prime}\right) \text {, }
$$

where $\mathcal{M}_{2}^{j}$ and $\mathcal{M}_{2}^{j^{\prime}}$ are both non-trivial; and

$$
P_{2, \text { weak }}^{j, s} V P_{2, \text { str }}^{j^{\prime}, t}=0
$$

where $\mathcal{M}_{2}^{j}$ is non-trivial and $\mathcal{M}_{2}^{j^{\prime}}$ is trivial or non-trivial.

Corollary 3.27. The operator PHP has a block structure defined by the projectors in (145) :

$$
\begin{gathered}
\text { PHP }=\sum_{\mathbf{m} \in \mathcal{M}_{1}} P_{\mathbf{m}} H P_{\mathbf{m}}+\sum_{j, \text { trivial }} \sum_{t} P_{2, s t r}^{j, t} H P_{2, s t r}^{j, t}+ \\
\sum_{j, \text { non-trivial }}\left(\sum_{s} P_{2, \text { weak }}^{j, s} H P_{2, \text { weak }}^{j, s}+\sum_{t} P_{2, s t r}^{j, t} H P_{2, \text { str }}^{j, t}\right) .
\end{gathered}
$$

Proof. Relations (146) follow from the definition of $\mathcal{M}_{1}$ and the obvious inequality $Q<k^{\delta} / 3$. Similar consideration yields (147)-(149) for $j \neq j^{\prime}$. Further we assume $j=j^{\prime}$.

The relation (147) follows from the definition of a cluster and the fact that each $\tilde{\mathcal{M}}_{2, s t r}^{j, t}$ belongs to $k^{\delta}$-neighborhoods of $\mathcal{M}_{2, s t r}^{j, t}$

Let us prove (148). Suppose (148) does not hold. Then, there is $\mathbf{m} \in \mathcal{M}_{2, \text { weak }}^{j, s}$, $\mathbf{m}^{\prime} \in \mathcal{M}_{2, \text { weak }}^{j, s^{\prime}}$, such that $V_{\mathbf{m}-\mathbf{m}^{\prime}} \neq 0$. Since $\mathbf{m}, \mathbf{m}^{\prime} \in \mathcal{M}_{2}^{j}$, we have $\mathbf{m}-\mathbf{m}^{\prime}=c \mathbf{q}$, where $\mathbf{q}$ is the direction of $\mathcal{M}_{2}^{j}$. Property 2) of potential $V$ implies that $c$ is an integer. Now, using the definition of $\mathcal{M}_{2}^{j, s}$, we obtain $s=s^{\prime}$. This contradicts to the assumption $s \neq s^{\prime}$ and, hence, proves (148).

Let us prove (149). Indeed, if $\mathcal{M}_{2, \text { weak }}^{j, s}$ is connected by $V$ with the main body of $\tilde{\mathcal{M}}_{2, s t r}^{j, t}$, then, by construction, such $\mathcal{M}_{2, \text { weak }}^{j, s}$ belongs to $\tilde{\mathcal{M}}_{2, s t r}^{j, t}$. If $\mathcal{M}_{2, \text { weak }}^{j, s}$ is connected by $V$ with a branch of $\tilde{\mathcal{M}}_{2, s t r}^{j, t}$, which is another weakly resonant $\mathcal{M}_{2, \text { weak }}^{j, s^{\prime}}$, then, considering as in the proof of (148), we obtain that this is the same branch. Therefore, again, $\mathcal{M}_{2, \text { weak }}^{j, s} \subset \tilde{\mathcal{M}}_{2, s t r}^{j, t}$. 
Since the summation in (145) is only over $\mathcal{M}_{2, \text { weak }}^{j, s}$ which are disjoint from $\tilde{\mathcal{M}}_{2, s t r}^{j, t}$, we arrive to contradiction. Thus, (149) is proven.

Since (34) holds for any $\mathbf{m} \in \tilde{\Omega}(\delta) \backslash\{0\}$ (see (33)), we have $\mathcal{M}\left(\varphi_{0}\right) \cap \tilde{\Omega}(\delta)=\emptyset$. This means that the $\||\cdot|||$-distance between $\tilde{\mathcal{M}}\left(\varphi_{0}\right)$ and $\Omega(\delta)$ is no less than $3 k^{\delta}$. Hence,

$$
P(\delta) V P_{\mathbf{m}}=P(\delta) V P_{2, w e a k}^{j, s}=P(\delta) V P_{2, s t r}^{j, t}=0 .
$$

We conclude this subsection with the following corollaries.

Lemma 3.28. Let $\varphi_{0} \in \omega^{(1)}(k, 8)$. Then, the operator $\left(P\left(H\left(\vec{\varkappa}^{(1)}(\varphi)\right)-k^{2} I\right) P\right)^{-1}$ has no more than $64 k^{4 r_{1}}$ poles in the disk $\left|\varphi-\varphi_{0}\right|<2 k^{-2-\delta(40 \mu+1)}$. The following estimate holds:

$$
\left\|\left(P\left(H\left(\vec{\varkappa}^{(1)}(\varphi)\right)-k^{2} I\right) P\right)^{-1}\right\|<k^{2 \mu r_{1}} \varepsilon_{0}^{-1}+c k^{-2} \varepsilon_{0}^{-2}+k^{-7} \varepsilon_{0}^{-4}, \quad \varepsilon_{0}=\min \left\{\varepsilon, k^{-2-41 \mu \delta}\right\},
$$

when $\varphi$ is in the smaller disk $\left|\varphi-\varphi_{0}\right|<k^{-2-\delta(40 \mu+1)}$, $\varepsilon$ being the distance from $\varphi$ to the nearest pole of the operator.

Corollary 3.29. If $\varepsilon=k^{-r_{1}^{\prime}}, r_{1}^{\prime} \geq \mu r_{1}$, then

$$
\begin{gathered}
\left\|\left(P\left(H\left(\vec{\varkappa}^{(1)}(\varphi)\right)-k^{2} I\right) P\right)^{-1}\right\|<\frac{1}{32} k^{4 r_{1}^{\prime}}, \\
\left\|\left(P\left(H\left(\vec{\varkappa}^{(1)}(\varphi)\right)-k^{2} I\right) P\right)^{-1}\right\|_{1}<\frac{1}{2} k^{4 r_{1}^{\prime}+4 r_{1}} .
\end{gathered}
$$

The first formula follows from (152). The second formula follows from the fact that the dimension of $P$ does not exceed $16 k^{4 r_{1}}$.

Proof. Indeed, the number of blocks in PHP (see (150) ) does not exceed $16 k^{4 r_{1}}$ (the number of elements in $\Omega\left(r_{1}\right)$ ). The resolvent of each block has no more than four poles. Therefore, the resolvent of PHP has no more than $64 k^{4 r_{1}}$ poles. Using Lemmas 3.21, 3.22, 3.23, 3.24, 3.25 and, using that $p_{\mathbf{m}}>k^{-2 \mu r_{1}}$ in (100), we obtain the lemma.

\subsubsection{Resonant and Nonresonant Sets for Step II}

We divide $[0,2 \pi)$ into $\left[2 \pi k^{2+\delta(40 \mu+1)}\right]+1$ intervals $\Delta_{l}^{(1)}$ with the length not bigger than $k^{-2-\delta(40 \mu+1)}$. If a particular interval belongs to $\mathcal{O}^{(1)}(k, 8)$ we ignore it; otherwise, let $\varphi_{0}^{(l)}$ be a point inside the $\Delta_{l}^{(1)}, \varphi_{0}^{(l)} \notin \mathcal{O}^{(1)}(k, 8)$. Let

$$
\mathcal{W}_{l}^{(1)}=\left\{\varphi \in \mathcal{W}^{(1)}:\left|\varphi-\varphi_{0}^{(l)}\right|<2 k^{-2-\delta(40 \mu+1)}\right\}
$$

Clearly, neighboring sets $\mathcal{W}_{l}^{(1)}$ overlap (because of the multiplier 2 in the inequality), they cover the $2 k^{-2-\delta(40 \mu+1)}$-neighborhood of $\omega^{(1)}(k, \delta, 8)$. We denote this neighborhood by 
$\hat{\mathcal{W}}^{(1)}(k, 2)$. For each $\varphi$ in the neighborhood there is a $l$ such that $\left|\varphi-\varphi_{0}^{(l)}\right|<k^{-2-\delta(40 \mu+1)}$. We consider the poles of the operator $\left(P\left(\varphi_{0}^{(l)}\right)\left(H\left(\vec{\varkappa}^{(1)}(\varphi)\right)-k^{2}\right) P\left(\varphi_{0}^{(l)}\right)\right)^{-1}$ in a $\mathcal{W}_{l}^{(1)}$ and denote them by $\varphi_{l m}, m=1, \ldots, M_{l}$. By Lemma 3.28, $M_{l}<64 k^{4 r_{1}}$. Next, let $\mathcal{O}_{l m}^{(2)}$ be the disc of the radius $k^{-r_{1}^{\prime}}$ around $\varphi_{l m}, r_{1}^{\prime}>\mu r_{1}$.

Definition 3.30. The set

$$
\mathcal{O}^{(2)}=\cup_{l m} \mathcal{O}_{l m}^{(2)}
$$

we call the second resonant set. The set

$$
\mathcal{W}^{(2)}=\hat{\mathcal{W}}^{(1)}(k, 2) \backslash \mathcal{O}^{(2)}
$$

is called the second nonresonant set. The set

$$
\omega^{(2)}=\mathcal{W}^{(2)} \cap[0,2 \pi)
$$

is called the second real nonresonant set.

Lemma 3.31. Let $r_{1}^{\prime}>\mu r_{1}, \varphi \in \mathcal{W}^{(2)}$ and $\varkappa \in \mathbb{C}:\left|\varkappa-\varkappa^{(1)}(\varphi)\right|<k^{-4 r_{1}^{\prime}-1-\delta}$. Then,

$$
\begin{gathered}
\left\|\left(P\left(H(\vec{\varkappa}(\varphi))-k^{2} I\right) P\right)^{-1}\right\|<k^{4 r_{1}^{\prime}}, \\
\left\|\left(P\left(H(\vec{\varkappa}(\varphi))-k^{2} I\right) P\right)^{-1}\right\|_{1}<k^{4 r_{1}^{\prime}+4 r_{1}},
\end{gathered}
$$

where $P$ is the projection (145) corresponding to the interval $\Delta_{l}^{(1)}$ containing $\Re \varphi$.

Proof. For $\vec{\varkappa}=\vec{\varkappa}^{(1)}(\varphi)$ the lemma follows immediately from the definition of $\mathcal{W}^{(2)}$ and Corollary 3.29. Considering the Hilbert identity, it is easy to see that estimates (153) and (154) are stable with respect to perturbation of $\varkappa^{(1)}$ of order $k^{-4 r_{1}^{\prime}-1-\delta}$. This stability ensure (158) and (159).

By total size of the set $\mathcal{O}^{(2)}$ we mean the sum of the sizes of its connected components.

Lemma 3.32. Let $r_{1}^{\prime} \geq(\mu+4) r_{1}$. Then, the size of each connected component of $\mathcal{O}^{(2)}$ is less than $128 k^{4 r_{1}-r_{1}^{\prime}}$. The total size of $\mathcal{O}^{(2)}$ is less than $10^{3} k^{2+\delta(40 \mu+1)+4 r_{1}-r_{1}^{\prime}}$, where $2+\delta(40 \mu+1)+4 r_{1}-r_{1}^{\prime}<0$.

Corollary 3.33. If a connected component of $\mathcal{O}^{(2)}$ intersects $[0,2 \pi)$ or its $\frac{1}{2} k^{-2-\delta(40 \mu+1)}$ neighborhood, then it is strictly inside $\tilde{\mathcal{W}}^{(1)}$.

Proof. Indeed, each set $\mathcal{W}_{l}^{(1)}$ contains no more than $64 k^{4 r_{1}} \operatorname{discs} \mathcal{O}_{l m}$. Therefore, the total size of $\mathcal{O}^{(2)} \cap \mathcal{W}_{l}^{(1)}$ is less than $128 k^{-r_{1}^{\prime}+4 r_{1}}$. Considering that $128 k^{-r_{1}^{\prime}+4 r_{1}}$ is much smaller than the length of $\Delta_{l}^{(1)}$, we obtain that there is no connected components which go across the whole set $\mathcal{W}_{l}^{(1)}$ and the size of each connected component of $\mathcal{O}^{(2)}$ is less than 
$128 k^{4 r_{1}-r_{1}^{\prime}}$. Considering that $l<7 k^{2+\delta(40 \mu+1)}$, we obtain the required estimate for the total size of $\mathcal{O}^{(2)}$.

We will also need the estimates for the resolvent in the neighborhood of $\mathbf{m}=0$. Let $C_{2}$ be a circle in the complex plane:

$$
C_{2}=\left\{z \in \mathbb{C}:\left|z-k^{2}\right|=\frac{1}{2} k^{-4 r_{1}^{\prime}}\right\} .
$$

Using the definition of $\vec{\varkappa}^{(1)}(\varphi)$ we obtain the following lemma.

Lemma 3.34. Let $\varphi \in \mathcal{W}_{l}^{(1)}$. Then,

$$
\left\|\left(P(\delta)\left(H\left(\vec{\varkappa}^{(1)}(\varphi)\right)-z\right) P(\delta)\right)^{-1}\right\| \leq 8 k^{4 r_{1}^{\prime}} .
$$

The proof is completely analogous to the proof of corresponding Lemma 3.21 from [1] and we omit it here.

Let

$$
\tilde{P}\left(\varphi_{0}^{(l)}\right):=P\left(\varphi_{0}^{(l)}\right)+P(\delta),
$$

Lemma 3.35. Let $\varphi$ be in the $k^{-r_{1}^{\prime}-\delta}$-neighborhood of $\mathcal{W}^{(2)} \cap W_{l}^{(1)}$ and $P, \tilde{P}$ be constructed for the interval $\Delta_{l}^{(1)}$ containing $\Re \varphi$. Then,

$$
\begin{gathered}
P V P(\delta)=0, \\
\tilde{P} H\left(\vec{\varkappa}^{(1)}(\varphi)\right) \tilde{P}=P(\delta) H P(\delta)+P H P \\
\left\|\left(\tilde{P}\left(H\left(\vec{\varkappa}^{(1)}(\varphi)\right)-z\right) \tilde{P}\right)^{-1}\right\| \leq 16 k^{4 r_{1}^{\prime}}, \quad \text { when } z \in C_{2} .
\end{gathered}
$$

Proof. Formula (162) follows from (145) and (151). Using (161) and (162), we obtain (163). We notice that the statement of the Lemma 3.31 still holds (up to the multiplier 2 at the r.h.s.), when we use $z \in C_{2}$ instead of $k^{2}$. We also use Lemma 3.34. At last, considering from the beginning the discs $\mathcal{O}_{j m}^{(2)}$ with radius $\frac{1}{2} k^{-r_{1}^{\prime}}$ instead of $k^{-r_{1}^{\prime}}$ one can easily see that similar estimates (up to another multiplier 2) hold in $k^{-r_{1}^{\prime}-\delta}$-neighborhood of $\mathcal{W}^{(2)}$.

\section{Step II}

\subsection{Operator $H^{(2)}$. Perturbation Formulas}

Let $P\left(r_{1}\right)$ be an orthogonal projector onto $\Omega\left(r_{1}\right):=\left\{\mathbf{m}:\left\|\vec{p}_{\mathbf{m}}\right\| \mid \leq k^{r_{1}}\right\}$ and $H^{(2)}=$ $P\left(r_{1}\right) H P\left(r_{1}\right)$. From now on we assume

$$
r_{1}^{\prime}=40 \mu r_{1}+2, \quad 2<r_{1}<k^{\delta / 8} .
$$


Let $\varphi$ be in the $k^{-r_{1}^{\prime}-\delta}$-neighborhood of $\mathcal{W}^{(2)} \cap W_{l}^{(1)}$. We consider $H^{(2)}\left(\vec{\varkappa}^{(1)}(\varphi)\right)$ as a perturbation of

$$
\tilde{H}^{(1)}=\tilde{P} H\left(\vec{\varkappa}^{(1)}(\varphi)\right) \tilde{P}+\left(P\left(r_{1}\right)-\tilde{P}\right) H_{0}\left(\vec{\varkappa}^{(1)}(\varphi)\right)\left(P\left(r_{1}\right)-\tilde{P}\right)
$$

where $\tilde{P}$ is defined in (161) and corresponds to the interval $\Delta_{l}^{(1)}$ containing $\Re \varphi$. By (163) and (150), the first term on the right-hand side of (166) has a block structure. The second term in (166) is, obviously, diagonal. Thus, $\tilde{H}^{(1)}$ has a block-diagonal structure. Let $W$ be the perturbation of $\tilde{H}^{(1)}$, i.e, $W=H^{(2)}-\tilde{H}^{(1)}$. It is easy to see that:

$$
W=P\left(r_{1}\right) V P\left(r_{1}\right)-\tilde{P} V \tilde{P} .
$$

By analogy with (39), (40),

$$
\begin{gathered}
g_{r}^{(2)}(\vec{\varkappa}):=\frac{(-1)^{r}}{2 \pi i r} \operatorname{Tr} \oint_{C_{2}}\left(W\left(\tilde{H}^{(1)}(\vec{\varkappa})-z I\right)^{-1}\right)^{r} d z, \\
G_{r}^{(2)}(\vec{\varkappa}):=\frac{(-1)^{r+1}}{2 \pi i} \oint_{C_{2}}\left(\tilde{H}^{(1)}(\vec{\varkappa})-z I\right)^{-1}\left(W\left(\tilde{H}^{(1)}(\vec{\varkappa})-z I\right)^{-1}\right)^{r} d z .
\end{gathered}
$$

Next theorem is the analogue of Theorem 4.1 from [1].

Theorem 4.1. Suppose $\varphi$ is in the real $k^{-r_{1}^{\prime}-\delta}$-neighborhood of $\omega^{(2)}(k, \delta, \tau)$ and $\varkappa \in \mathbb{R}$, $\left|\varkappa-\varkappa^{(1)}(\varphi)\right| \leq k^{-4 r_{1}^{\prime}-1-\delta}, \vec{\varkappa}=\varkappa(\cos \varphi, \sin \varphi)$. Then, for sufficiently large $k>k_{1}(V, \delta, \tau)$ there exists a single eigenvalue of $H^{(2)}(\vec{\varkappa})$ in the interval $\varepsilon_{2}(k, \delta, \tau)=\left(k^{2}-\frac{1}{2} k^{-4 r_{1}^{\prime}}, k^{2}+\frac{1}{2} k^{-4 r_{1}^{\prime}}\right)$. It is given by the absolutely converging series:

$$
\lambda^{(2)}(\vec{\varkappa})=\lambda^{(1)}(\vec{\varkappa})+\sum_{r=2}^{\infty} g_{r}^{(2)}(\vec{\varkappa}) .
$$

For coefficients $g_{r}^{(2)}(\vec{\varkappa})$ the following estimates hold:

$$
\left|g_{r}^{(2)}(\vec{\varkappa})\right|<k^{-k^{\delta}(2 Q)^{-1}} k^{-\delta_{*} r / 16} .
$$

The corresponding spectral projection is given by the series:

$$
\mathcal{E}^{(2)}(\vec{\varkappa})=\mathcal{E}^{(1)}(\vec{\varkappa})+\sum_{r=1}^{\infty} G_{r}^{(2)}(\vec{\varkappa}),
$$

$\mathcal{E}^{(1)}(\vec{\varkappa})$ being the spectral projection of $H^{(1)}(\vec{\varkappa})$. The operators $G_{r}^{(2)}(\vec{\varkappa})$ satisfy the estimates:

$$
\begin{gathered}
\left\|G_{r}^{(2)}(\vec{\varkappa})\right\|_{1}<k^{-k^{\delta}(4 Q)^{-1}} k^{-\delta_{*} r / 16} . \\
G_{r}^{(2)}(\vec{\varkappa})_{\mathbf{s s}^{\prime}}=0, \quad \text { if } 10 r k^{\delta_{*}}<\left\|\left|\vec { p } _ { \mathbf { s } } \left\|\left|+\left\|\vec{p}_{\mathbf{s}^{\prime}}\right\|\right|\right.\right.\right.
\end{gathered}
$$


Corollary 4.2. For the perturbed eigenvalue and its spectral projection the following estimates hold:

$$
\begin{gathered}
\lambda^{(2)}(\vec{\varkappa})=\lambda^{(1)}(\vec{\varkappa})+O\left(k^{-k^{\delta}(2 Q)^{-1}}\right), \\
\left\|\mathcal{E}^{(2)}(\vec{\varkappa})-\mathcal{E}^{(1)}(\vec{\varkappa})\right\|_{1}<k^{-k^{\delta}(4 Q)^{-1}}, \\
\left|\mathcal{E}^{(2)}(\vec{\varkappa})_{\mathbf{s s}^{\prime}}\right|<k^{-d^{(2)}\left(\mathbf{s}, \mathbf{s}^{\prime}\right)}, \quad \text { when }\left\|\left|\vec{p}_{\mathbf{s}} \|\right|>k^{\delta} \text { or }\right\|\left|\vec{p}_{\mathbf{s}^{\prime}} \|\right|>k^{\delta}, \\
d^{(2)}\left(\mathbf{s}, \mathbf{s}^{\prime}\right)=\frac{\delta_{*}}{160}\left(\left\|\left|\vec { p } _ { \mathbf { s } } \left\|\left|+\left\|\left|\vec{p}_{\mathbf{s}^{\prime}} \|\right|\right) k^{-\delta_{*}}+k^{\delta}(4 Q)^{-1} .\right.\right.\right.\right.\right.
\end{gathered}
$$

Formulas (175) and (176) easily follow from (170), (172) and (171) and (173). The estimate (177) follows from (172), (173) and (174). Indeed, using these estimates, we obtain $\left|\left(\mathcal{E}^{(2)}(\vec{\varkappa})-\mathcal{E}^{(1)}(\vec{\varkappa})\right)_{\mathbf{s s}^{\prime}}\right|<k^{-d^{(2)}\left(\mathbf{s}, \mathbf{s}^{\prime}\right)}$. Considering that $\mathcal{E}^{(1)}(\vec{\varkappa})_{\mathbf{s s}^{\prime}}=0$ when $\left\|\left|\vec{p}_{\mathbf{s}} \|\right|>\right.$ $k^{\delta}$ or $\left\|\left|\vec{p}_{\mathbf{s}^{\prime}} \|\right|>k^{\delta}\right.$, we arrive at (177).

Proof. Let $P^{\prime}:=P\left(r_{1}\right)-\tilde{P}$. By (166), (167),

$$
\tilde{H}^{(1)}\left(\vec{\varkappa}^{(1)}(\varphi)\right):=\tilde{P} H\left(\vec{\varkappa}^{(1)}(\varphi)\right) \tilde{P}+P^{\prime} H_{0}\left(\vec{\varkappa}^{(1)}(\varphi)\right) P^{\prime}, \quad W:=P^{\prime} V P^{\prime}+P^{\prime} V \tilde{P}+\tilde{P} V P^{\prime} .
$$

We will often omit $\vec{\varkappa}^{(1)}(\varphi)$ in the arguments when it cannot lead to confusion. By (164), we have

$$
\left\|\left(\tilde{H}^{(1)}-z I\right)^{-1}\right\|<16 k^{4 r_{1}^{\prime}} .
$$

Let us consider the perturbation series

$$
\left(H^{(2)}-z\right)^{-1}=\sum_{r=0}^{\infty}\left(\tilde{H}^{(1)}-z\right)^{-1}\left(-W\left(\tilde{H}^{(1)}-z\right)^{-1}\right)^{r} .
$$

Put

$$
\tilde{A}:=-\left(\tilde{H}^{(1)}-z\right)^{-1} W\left(\tilde{H}^{(1)}-z\right)^{-1} .
$$

To check the convergence it is enough to show that

$$
\|\tilde{A}\|<k^{-\delta_{*} / 8}
$$

Estimates (178) and (180) yield

$$
\left\|\left(H^{(2)}-z I\right)^{-1}\right\|<32 k^{4 r_{1}^{\prime}} .
$$

To prove (180) it suffices to check

$$
\begin{gathered}
\left\|P^{\prime} \tilde{A} P^{\prime}\right\|<4\|V\| k^{-\delta_{*} / 2+215 \mu \delta} . \\
\left\|P^{\prime} \tilde{A} \tilde{P}\right\|<2\|V\| k^{-\delta_{*} / 4+214 \mu \delta} .
\end{gathered}
$$

Let us prove (182). By construction, $P^{\prime}=P_{\text {nonres }}+P_{\text {triv,weak }}$, where $P_{\text {nonres }}$ is the projection on $\Omega\left(r_{1}\right) \backslash \mathcal{M}$ and $P_{\text {triv,weak }}$ is the projection on those weak points in trivial clusters 
$\mathcal{M}_{2}^{j}$, which do not belong to $\frac{1}{3} k^{\delta}$-neighborhoods of strong points. Using Lemmas 3.21 and 3.23, we easily obtain

$$
\left\|P_{\text {nonres }} \tilde{A} P_{\text {nonres }}\right\|<4\|V\| k^{-2 \delta_{*}},\left\|P_{\text {nonres }} \tilde{A} P_{\text {triv,weak }}\right\|,\left\|P_{\text {triv,weak }} \tilde{A} P_{\text {nonres }}\right\|<2\|V\| k^{-\delta_{*}+214 \mu \delta} \text {. }
$$

To obtain $\left\|P_{\text {triv,weak }} \tilde{A} P_{\text {triv,weak }}\right\|<2\|V\| k^{-\delta_{*} / 2+215 \mu \delta}$, we use the arguments from the proof of Lemma 3.24. Namely, if $\mathcal{M}_{2}^{j}$ corresponds to the first option in the Definition 3.15 then $P_{\text {triv,weak }} \tilde{A} P_{\text {triv,weak }}=0$. If $\mathcal{M}_{2}^{j}$ corresponds to the second option in the Definition 3.15] then the inequality opposite to (128) is valid for any pair $\mathbf{m}, \mathbf{m}^{\prime} \in \mathcal{M}_{2, \text { triv,weak }}^{j}$. Combining this with Lemma 3.23 we get the sought estimate. Thus, (182) is proven.

Next, we prove (183). By (146)-(148), it is enough to check

$$
\begin{gathered}
\left\|P^{\prime} \tilde{A} P_{\mathbf{m}}\right\|<8\|V\| k^{-\delta_{*}+214 \mu \delta}, \mathbf{m} \in \mathcal{M}_{1} \cup \mathbf{0}, \text { where } P_{\mathbf{0}}:=P(\delta), \\
\left\|P^{\prime} \tilde{A} P_{2, \text { triv,str }}^{j, s}\right\|<2\|V\| k^{-\delta_{*} / 4+214 \mu \delta}, \\
\left\|P^{\prime} \tilde{A} P_{2, \text { nontriv,weak }}^{j, s}\right\|<2\|V\| k^{-\delta_{*}+214 \mu \delta} \\
\left\|P^{\prime} \tilde{A} P_{2, \text { nontriv, strong }}^{j, s}\right\|<2\|V\| k^{-\delta_{*} / 4+214 \mu \delta}
\end{gathered}
$$

To prove (185) we represent $\left(\tilde{H}^{(1)}-z\right)^{-1} P_{\mathbf{m}}$ as follows:

$$
\begin{aligned}
& \left(\tilde{H}^{(1)}-z\right)^{-1} P_{\mathbf{m}}=\sum_{r=0}^{R_{0}}\left(-\left(H_{0}-z\right)^{-1} P_{\mathbf{m}} V P_{\mathbf{m}}\right)^{r}\left(H_{0}-z\right)^{-1} P_{\mathbf{m}}+ \\
& \left(-\left(H_{0}-z\right)^{-1} P_{\mathbf{m}} V P_{\mathbf{m}}\right)^{R_{0}+1}\left(\tilde{H}^{(1)}-z\right)^{-1} P_{\mathbf{m}},
\end{aligned}
$$

where $R_{0}$ to be fixed later. Then,

$$
\begin{aligned}
& \left\|P^{\prime} V P_{\mathbf{m}}\left(\tilde{H}^{(1)}-z\right)^{-1}\right\| \leq \sum_{r=0}^{R_{0}}\left\|B_{r}\right\|+\left\|P^{\prime} V\left(\left(H_{0}-z\right)^{-1} P_{\mathbf{m}} V P_{\mathbf{m}}\right)^{R_{0}+1}\right\|\left\|\left(\tilde{H}^{(1)}-z\right)^{-1} P_{\mathbf{m}}\right\|, \\
& B_{r}:=P^{\prime} V\left(\left(H_{0}-z\right)^{-1} P_{\mathbf{m}} V P_{\mathbf{m}}\right)^{r}\left(H_{0}-z\right)^{-1} P_{\mathbf{m}} .
\end{aligned}
$$

Note that $B_{r}=P^{\prime} B_{r} P_{\mathbf{m}}$ and matrix elements $\left(B_{r}\right)_{\mathbf{j s}}$ are equal to zero if $\left\|\vec{p}_{\mathbf{j}}-\vec{p}_{\mathbf{s}}\right\| \mid>$ $Q(r+1)$ (see (32) ). Thus, the only non-trivial elements $\left(B_{r}\right)_{\mathbf{j s}}$ are such that

$$
\mathbf{j} \in \Omega\left(r_{1}\right) \backslash\left(\tilde{\mathcal{M}}\left(\varphi_{0}\right) \cup \Omega(\delta)\right), \quad \mathbf{s} \in \tilde{\mathcal{M}}_{\mathbf{m}}\left(\varphi_{0}\right) \cup \Omega(\delta), \quad\left|\left\|\vec{p}_{\mathbf{j}}-\vec{p}_{\mathbf{s}}\right\|\right| \leq Q(r+1) .
$$

Let $r: Q(r+1) \leq k^{\delta} / 6$. It follows that $\left(B_{r}\right)_{\mathbf{j s}}=0$ if $\mathbf{s}=\mathbf{m}$ or $\mathbf{s}=\mathbf{0}$, since such $\mathbf{s}$ have the distance greater than $\frac{1}{3} k^{\delta}$ from $\mathbf{j}$. If $\mathbf{s} \neq \mathbf{m}$ or $\mathbf{s} \neq 0$, then ||$\vec{\varkappa}^{(1)}(\varphi)+\left.\vec{p}_{\mathbf{s}}\right|_{\mathbb{R}} ^{2}-z \mid>\frac{1}{4} k^{\delta_{*}}$. Therefore, for $r: Q(r+1) \leq k^{\delta} / 6$ we have:

$$
\left\|B_{r}\right\| \leq\left(4\|V\| k^{-\delta_{*}}\right)^{r+1}, \quad\left\|P^{\prime} V\left(\left(H_{0}-z\right)^{-1} P_{\mathbf{m}} V P_{\mathbf{m}}\right)^{r+1}\right\| \leq\|V\|\left(4\|V\| k^{-\delta_{*}}\right)^{r+1} .
$$


Now, we fix $R_{0}:=\left[k^{\delta} /(6 Q)\right]-1$. Then the condition $Q(r+1) \leq k^{\delta} / 6$ is satisfied for all $r \leq R_{0}$ and

$$
\left\|P^{\prime} V P_{\mathbf{m}}\left(\tilde{H}^{(1)}-z\right)^{-1}\right\| \leq \sum_{r=0}^{R_{0}}\left(4\|V\| k^{-\delta_{*}}\right)^{r+1}+\|V\|\left(4\|V\| k^{-\delta_{*}}\right)^{R_{0}+1} 16 k^{4 r_{1}^{\prime}} .
$$

Assuming that $k$ is large enough (in particular, $\frac{\delta_{*} k^{\delta}}{6 Q}>5 r_{1}^{\prime}$ ) and using Lemma 3.23 , we obtain (185).

Let us prove (186). Noting that $P_{2, \text { triv,str }}^{j, s}$ is the projection into the $k^{\delta}$-neighborhood of a strongly resonant point and $V_{\mathbf{q}}=0$ when $\left\|\left|\vec{p}_{\mathbf{q}} \|\right|>Q\right.$, we conclude that we can insert a projection into the formula for $\tilde{A}$; namely $P^{\prime} \tilde{A} P_{2, s t r}^{j, s}=P^{\prime}\left(H_{0}-z\right)^{-1} V P^{\prime \prime}\left(\tilde{H}^{(1)}-z\right)^{-1}$, where $P^{\prime \prime}$ is the projection corresponding to the points situated outside the $\left(k^{\delta}-Q\right)$ neighborhood of a strongly resonant point. Using Lemmas 3.21 and 3.23 , we obtain $\left\|P^{\prime}\left(H_{0}-z\right)^{-1}\right\|<2 k^{214 \mu \delta}$. Using (120) with $d\left(\mathbf{m}, \mathbf{m}^{\prime}\right)>\left(k^{\delta}-Q\right) /(20 Q)$, and $\varepsilon_{0}=k^{-r_{1}^{\prime}}$, $\left(r_{1}^{\prime}<k^{\delta} \delta_{*} /(100 Q)\right)$, we obtain $\left\|P^{\prime \prime}\left(\tilde{H}^{(1)}-z\right)^{-1}\right\|<\|V\| k^{-\delta_{*} / 4}$. Now (186) easily follows.

Let us prove (187). Obviously, $P^{\prime} \tilde{A} P_{2, \text { nontriv,weak }}^{j, s}=P_{\text {nonres }} \tilde{A} P_{2, \text { nontriv,weak }}^{j, s}$. Using Lemmas 3.21 and 3.23, we arrive at (187).

The proof of (188) is analogous to the proof of (186), with (138) being used instead of $(120)$.

To prove (173) we consider the operator $A=W\left(\tilde{H}^{(1)}-z\right)^{-1}$ and represent it as $A=$ $A_{0}+A_{1}+A_{2}$, where $A_{0}=\left(P\left(r_{1}\right)-\mathcal{E}^{(1)}(\vec{\varkappa})\right) A\left(P\left(r_{1}\right)-\mathcal{E}^{(1)}(\vec{\varkappa})\right), A_{1}=\left(P\left(r_{1}\right)-\mathcal{E}^{(1)}(\vec{\varkappa})\right) A \mathcal{E}^{(1)}(\vec{\varkappa})$, $A_{2}=\mathcal{E}^{(1)}(\vec{\varkappa}) A\left(P\left(r_{1}\right)-\mathcal{E}^{(1)}(\vec{\varkappa})\right)$. Note that we have $\mathcal{E}^{(1)}(\vec{\varkappa}) W \mathcal{E}^{(1)}(\vec{\varkappa})=0$, because of (167). It is easy to see that by construction $A_{0}$ is holomorphic inside $C_{2}$ (see, e.g. Lemma 3.31 and Theorem 3.3). Hence,

$$
\oint_{C_{2}}\left(\tilde{H}^{(1)}-z\right)^{-1} A_{0}^{r} d z=0
$$

Therefore,

$$
G_{r}^{(2)}(\vec{\varkappa})=\frac{(-1)^{r}}{2 \pi i} \sum_{j_{1}, \ldots j_{r}=0,1,2, j_{1}^{2}+\ldots+j_{r}^{2} \neq 0} I_{j_{1} \ldots j_{r}}, \quad I_{j_{1} \ldots j_{r}}:=\oint_{C_{2}}\left(\tilde{H}^{(1)}-z\right)^{-1} A_{j_{1} \ldots . . A_{j_{r}} d z .}
$$

At least one of indices in each term is equal to 1 or 2 . Let us show that

$$
\left\|A_{2}\right\|_{1}<c k^{-k^{\delta}(2 Q)^{-1}} k^{214 \mu \delta} .
$$

First, we notice that $\mathcal{E}^{(1)} W\left(P\left(r_{1}\right)-\mathcal{E}^{(1)}\right)=\mathcal{E}^{(1)} W P^{\prime}$ by (167) and (151). It suffices to show that

$$
\left\|\mathcal{E}^{(1)} W P^{\prime}\right\|_{1}<c k^{-k^{\delta}(2 Q)^{-1}}
$$


since $\left\|P^{\prime}\left(\tilde{H}^{(1)}-z\right)^{-1}\right\|=\left\|P^{\prime}\left(H_{0}-z\right)^{-1}\right\|<2 k^{214 \mu \delta}$ for $z \in C_{2}$. Indeed,

$$
\left(\mathcal{E}^{(1)} W P^{\prime}\right)_{\mathbf{s s}^{\prime}}=\sum_{\mathbf{s}^{\prime \prime}:\left\|\vec{p}_{\mathbf{s}^{\prime \prime}}\right\|\left|\leq k^{\delta},\left\|\mid \vec{p}_{\mathbf{s}^{\prime \prime}-\mathbf{s}^{\prime}}\right\| \leq Q\right.} \mathcal{E}_{\mathbf{s s}^{\prime \prime}}^{(1)} W_{\mathbf{s}^{\prime \prime}-\mathbf{s}^{\prime}}
$$

when $\left\|\left|\vec{p}_{\mathbf{s}^{\prime}} \|\right|>k^{\delta}\right.$ and it is equal to zero otherwise. Hence,

$$
\left|\left(\mathcal{E}^{(1)} W P^{\prime}\right)_{\mathbf{s s}^{\prime}}\right| \leq\|W\| \sum_{\mathbf{s}^{\prime \prime}: k^{\delta}-Q \leq\left\|\vec{p}_{\mathbf{s}^{\prime \prime}}\right\| \mid \leq k^{\delta}} \mathcal{E}_{\mathbf{s s}^{\prime \prime}}^{(1)}
$$

if $\left\|\left|\vec{p}_{\mathbf{s}^{\prime}} \|\right|<k^{\delta}+Q\right.$ and zero otherwise. Using (50), we obtain

$$
\left|\left(\mathcal{E}^{(1)} W P^{\prime}\right)_{\mathbf{s s}^{\prime}}\right|<c k^{4 \delta} \max _{\left\|\vec{p}_{\mathbf{s}^{\prime \prime}}\right\| \mid>k^{\delta}-Q} k^{-d^{(1)}\left(\mathbf{s}, \mathbf{s}^{\prime \prime}\right)} .
$$

It easily follows:

$$
\left|\left(\mathcal{E}^{(1)} W P^{\prime}\right)_{\mathbf{s s}^{\prime}}\right|<c k^{4 \delta} k^{-(1-40 \mu \delta)\left(k^{\delta} Q^{-1}-1+\left\|\vec{p}_{\mathbf{s}}\right\| Q^{-1}\right)}
$$

when $\left\|\left|\vec{p}_{\mathbf{s}^{\prime}} \|\right|<k^{\delta}+Q\right.$, and zero otherwise. It follows $\| \mathcal{E}^{(1)} W P^{\prime} \|<c k^{-k^{\delta}(2 Q)^{-1}}$. Considering that $\mathcal{E}^{(1)}$ is a one-dimensional projection, we obtain the same estimate for $\mathbf{S}_{\mathbf{1}}$-norm, namely, (193). Thus, we have proved (192). Let us estimate $I_{j_{1} \ldots j_{r}}$. Suppose one of the indices is equal to 2. Substituting (192) into (191) and taking into account (180), (178), we obtain:

$$
\left\|I_{j_{1} \ldots j_{r}}\right\|<c k^{-k^{\delta}(2 Q)^{-1}} k^{214 \mu \delta} k^{-\frac{1}{8} \delta_{*}\left(\frac{r}{2}-1\right)} k^{4 r_{1}^{\prime}}<k^{-k^{\delta}(4 Q)^{-1}} k^{-\delta_{*} r / 16} .
$$

(More precisely, our $A_{2}$ splits the integrand in $I_{j_{1} \ldots j_{r}}$ into two parts; for each part we use (180) for every product of two $A_{j_{k}}$ and (178) for the last single $A_{j_{s}}$ or $\left(\tilde{H}^{(1)}-z\right)^{-1}$; we also take into account the length of the circle.) Note that the operator $A_{1}$ is always followed by $A_{2}$ unless $A_{1}$ occupies the very last position in the product. Thus, it remains to consider the case $A_{j_{1}} \ldots . A_{j_{r}}=A_{0}^{r-1} A_{1}$. It is easy to see that

$$
\left(\tilde{H}^{(1)}-z\right)^{-1} A_{0}^{r-1} A_{1}=\left(\left(\tilde{H}^{(1)}-\bar{z}\right)^{-1} A_{2}(\bar{z}) A_{0}^{r-1}(\bar{z})\right)^{*}
$$

This implies the estimate for this case too. Therefore,

$$
\left\|G_{r}^{(2)}(\vec{\varkappa})\right\|<k^{-k^{\delta}(4 Q)^{-1}} k^{-\delta_{*} r / 16} .
$$

The same estimate can be written for the $\mathbf{S}_{\mathbf{1}}$ norm of this operator, since $\mathcal{E}^{(1)}$ is onedimensional. 
Let us obtain the estimate for $g_{r}(\vec{\varkappa})$. Obviously,

$$
g_{r}^{(2)}(\vec{\varkappa})=\frac{(-1)^{r}}{2 \pi i r} \sum_{j_{1}, \ldots j_{r}=0,1,2, j_{1}^{2}+\ldots+j_{r}^{2} \neq 0} \operatorname{Tr} \oint_{C_{2}} A_{j_{1}} \ldots . . A_{j_{r}} d z .
$$

Note that each term contains both $A_{1}$ and $A_{2}$, since we compute the trace of the integral. Using (193), we obtain: $\left\|A_{1}\right\|_{1}<c b_{2}^{-1} k^{-k^{\delta}(2 Q)^{-1}}$, where $b_{2}$ is the radius of $C_{2}$. Combining this estimate with (192) and (180), (178), we obtain (171) for $r \geq 2$. Finally, applying (168) in the case $r=1$, we see that $g_{1}^{(2)}(\vec{\varkappa})=0$, since $\mathcal{E}^{(1)} W \mathcal{E}^{(1)}=0$.

To prove (174) it's enough to notice that the biggest block of $\tilde{H}^{(1)}$ has the size not greater than $2 k^{\delta_{*}}$.

It is easy to see that coefficients $g_{r}^{(2)}(\vec{\varkappa})$ and operators $G_{r}^{(2)}(\vec{\varkappa})$ can be analytically extended into the complex $k^{-r_{1}^{\prime}-\delta}$-neighborhood of $\omega^{(2)}$ (in fact, into $k^{-r_{1}^{\prime}-\delta}$-neighborhood of $\left.\mathcal{W}^{(2)}\right)$ as functions of $\varphi$ and to the complex $\left(k^{-4 r_{1}^{\prime}-1-\delta}\right)-$ neighborhood of $\varkappa=\varkappa^{(1)}(\varphi)$ as functions of $\varkappa$, estimates (171), (175) being preserved. Now, we use formulae (168), (170) to extend $\lambda^{(2)}(\vec{\varkappa})=\lambda^{(2)}(\varkappa, \varphi)$ as an analytic function. Obviously, series (170) is differentiable. Using Cauchy integral and Lemma 3.5 we get the following lemma.

Lemma 4.3. Under conditions of Theorem 4.1 the following estimates hold when $\varphi \in$ $\omega^{(2)}(k, \delta)$ or its complex $k^{-r_{1}^{\prime}-\delta}$-neighborhood and $\varkappa \in \mathbb{C}:\left|\varkappa-\varkappa^{(1)}(\varphi)\right|<k^{-4 r_{1}^{\prime}-1-\delta}$ :

$$
\begin{gathered}
\lambda^{(2)}(\vec{\varkappa})=\lambda^{(1)}(\vec{\varkappa})+O\left(k^{-k^{\delta}(2 Q)^{-1}}\right), \\
\frac{\partial \lambda^{(2)}}{\partial \varkappa}=\frac{\partial \lambda^{(1)}}{\partial \varkappa}+O\left(k^{-k^{\delta}(2 Q)^{-1}} k^{4 r_{1}^{\prime}+1+\delta}\right), \\
\frac{\partial \lambda^{(2)}}{\partial \varphi}=\frac{\partial \lambda^{(1)}}{\partial \varphi}+O\left(k^{-k^{\delta}(2 Q)^{-1}} k^{r_{1}^{\prime}+\delta}\right), \\
\frac{\partial^{2} \lambda^{(2)}}{\partial \varkappa^{2}}=\frac{\partial^{2} \lambda^{(1)}}{\partial \varkappa^{2}}+O\left(k^{-k^{\delta}(2 Q)^{-1}} k^{8 r_{1}^{\prime}+2+2 \delta}\right), \\
\frac{\partial^{2} \lambda^{(2)}}{\partial \varkappa \partial \varphi}=\frac{\partial^{2} \lambda^{(1)}}{\partial \varkappa \partial \varphi}+O\left(k^{-k^{\delta}(2 Q)^{-1}} k^{5 r_{1}^{\prime}+1+2 \delta}\right), \\
\frac{\partial^{2} \lambda^{(2)}}{\partial \varphi^{2}}=\frac{\partial^{2} \lambda^{(1)}}{\partial \varphi^{2}}+O\left(k^{-k^{\delta}(2 Q)^{-1}} k^{2 r_{1}^{\prime}+2 \delta}\right) .
\end{gathered}
$$

\subsection{Isoenergetic Surface for Operator $H^{(2)}$}

Lemma 4.4. 1. For every sufficiently large $\lambda, \lambda:=k^{2}$, and $\varphi$ in the real $\frac{1}{2} k^{-r_{1}^{\prime}-\delta_{-}}$ neighborhood of $\omega^{(2)}(k, \delta, \tau)$, there is a unique $\varkappa^{(2)}(\lambda, \varphi)$ in the interval $I_{1}:=$ $\left[\varkappa^{(1)}(\lambda, \varphi)-\frac{1}{2} k^{-4 r_{1}^{\prime}-1-\delta}, \varkappa^{(1)}(\lambda, \varphi)+\frac{1}{2} k^{-4 r_{1}^{\prime}-1-\delta},\right]$, such that

$$
\lambda^{(2)}\left(\vec{\varkappa}^{(2)}(\lambda, \varphi)\right)=\lambda, \quad \vec{\varkappa}^{(2)}(\lambda, \varphi):=\varkappa^{(2)}(\lambda, \varphi) \vec{\nu}(\varphi) .
$$


2. Furthermore, there exists an analytic in $\varphi$ continuation of $\varkappa^{(2)}(\lambda, \varphi)$ to the complex $\frac{1}{2} k^{-r_{1}^{\prime}-\delta}$-neighborhood of $\omega^{(2)}(k, \delta, \tau)$ such that $\lambda^{(2)}\left(\vec{\varkappa}^{(2)}(\lambda, \varphi)\right)=\lambda$. Function $\varkappa^{(2)}(\lambda, \varphi)$ can be represented as $\varkappa^{(2)}(\lambda, \varphi)=\varkappa^{(1)}(\lambda, \varphi)+h^{(2)}(\lambda, \varphi)$, where

$$
\begin{gathered}
\left|h^{(2)}(\varphi)\right|=O\left(k^{-k^{\delta}(2 Q)^{-1}} k^{-1}\right), \\
\frac{\partial h^{(2)}}{\partial \varphi}=O\left(k^{-k^{\delta}(2 Q)^{-1}} k^{r_{1}^{\prime}+\delta-1}\right), \quad \frac{\partial^{2} h^{(2)}}{\partial \varphi^{2}}=O\left(k^{-k^{\delta}(2 Q)^{-1}} k^{2 r_{1}^{\prime}+2 \delta-1}\right) .
\end{gathered}
$$

Proof. The proof is completely analogous to that of Lemma 3.11, estimates (196) -(201) being used.

Let us consider the set of points in $\mathbb{R}^{2}$ given by the formula: $\vec{\varkappa}=\vec{\varkappa}^{(2)}(\varphi), \quad \varphi \in$ $\omega^{(2)}(k, \delta, \tau)$. By Lemma 4.4 this set of points is a slight distortion of $\mathcal{D}_{1}$. All the points of this curve satisfy the equation $\lambda^{(2)}\left(\vec{\varkappa}^{(2)}(\varphi)\right)=k^{2}$. We call it isoenergetic surface of the operator $H^{(2)}$ and denote by $\mathcal{D}_{2}$.

\subsection{Preparation for Step III - Geometric Part. Properties of the Quasiperiodic Lattice}

Let

$$
\mathcal{S}\left(k, \varepsilon_{0}\right)=\left\{\vec{\varkappa} \in \mathbb{R}^{2}:\left\|\left(H^{(1)}(\vec{\varkappa})-k^{2}\right)^{-1}\right\|>\varepsilon_{0}^{-1}\right\} .
$$

In this section we prove that the number of the lattice points $\vec{\varkappa}_{0}+\vec{p}_{\mathbf{m}},\left\|\left|\vec{p}_{\mathbf{m}} \|\right|<k^{r_{1}}\right.$ in $\mathcal{S}\left(k, \varepsilon_{0}\right)$ does not exceed $C k^{\frac{2 r_{1}}{3}+1}$ when $\varepsilon_{0}$ is sufficiently small and $\vec{\varkappa}_{0}$ is fixed. For this we split $\mathcal{S}$ into two subsets: "non-resonant" and "resonant", the non-resonant set being just a vicinity of $\mathcal{D}_{1}\left(k^{2}\right)$. An estimate for the number of lattice points in the non-resonant set is proven in Lemma 4.8. Estimates for the number of lattice points in different types of resonant sets are proven in Lemmas 4.10, 4.12, 4.13, 4.16, These estimates play an important role in the further construction.

\subsubsection{General Lemmas}

We consider $\vec{p}_{\mathbf{m}}=2 \pi\left(\mathbf{s}_{1}+\alpha \mathbf{s}_{2}\right)$ with integer vectors $\mathbf{s}_{j}$ such that $\left|\mathbf{s}_{j}\right| \leq 4 k^{r_{1}}$.

It is easy to see that there exists a pair $(q, p) \in \mathbb{Z}^{2}$ such that $0<q \leq 4 k^{r_{1}}$ and

$$
|\alpha q+p| \leq \frac{1}{4} k^{-r_{1}}
$$

We choose a pair $(p, q)$ which gives the best approximation. In particular, $p$ and $q$ are mutually prime. Put $\epsilon_{q}:=\alpha+\frac{p}{q}$. We have (see (27), (206))

$$
k^{-2 r_{1} \mu} \leq\left|\epsilon_{q}\right| \leq \frac{1}{4} q^{-1} k^{-r_{1}} .
$$


Here we assume that $k$ is large enough (depending on $\alpha$ only) so that (27) ensures the lower inequality in (207). In what follows we will often make similar assumptions without additional remarks.

We write any $\mathbf{s}_{2}$ in the form

$$
\mathbf{s}_{2}=q \mathbf{s}_{2}^{\prime}+\mathbf{s}_{2}^{\prime \prime}
$$

with integer vectors $\mathbf{s}_{2}^{\prime}$ and $\mathbf{s}_{2}^{\prime \prime}, 0 \leq\left(\mathbf{s}_{2}^{\prime \prime}\right)_{j}<q$ for $j=1,2$. Hence, $\left|\left(\mathbf{s}_{2}^{\prime}\right)_{j}\right| \leq 4 k^{r_{1}} / q+1$. It follows

$$
(2 \pi)^{-1} \vec{p}_{\mathbf{m}}=\left(\mathbf{s}_{1}-p \mathbf{s}_{2}^{\prime}\right)+\left(-\frac{p}{q} \mathbf{s}_{2}^{\prime \prime}+\epsilon_{q} \mathbf{s}_{2}^{\prime \prime}\right)+\epsilon_{q} q \mathbf{s}_{2}^{\prime}
$$

Denote $\mathbf{s}:=\mathbf{s}_{1}-p \mathbf{s}_{2}^{\prime}$. Then $|\mathbf{s}| \leq 8 k^{r_{1}}$. The number of different vectors $\tilde{\mathbf{s}}:=-\frac{p}{q} \mathbf{s}_{2}^{\prime \prime}+\epsilon_{q} \mathbf{s}_{2}^{\prime \prime}$ is not greater than $(2 q)^{2}$. For each fixed pair $\tilde{\mathbf{s}}$, $\mathbf{s}$ we obtain a lattice parameterized by $\mathbf{s}_{2}^{\prime}$. We call this lattice a cluster corresponding to given $\tilde{\mathbf{s}}$, $\mathbf{s}$. Each cluster, obviously, is a square lattice with the step $\epsilon_{q} q$. It contains no more than $\left(9 k^{r_{1}} q^{-1}\right)^{2}$ elements, since $\left|\left(\mathbf{s}_{2}^{\prime}\right)_{j}\right| \leq 4 k^{r_{1}} q^{-1}+1, j=1,2$. The size of each cluster is less than $5\left|\epsilon_{q}\right| k^{r_{1}}$. If $\epsilon_{q}$ satisfies slightly stronger inequality, than (207) then clusters don't overlap, see the following lemma.

Lemma 4.5. Suppose that $\epsilon_{q}$ satisfies the inequality

$$
\left|\epsilon_{q}\right| \leq \frac{1}{64} q^{-1} k^{-r_{1}}
$$

Then, the size of each cluster is less that $\frac{1}{8 q}$. The distance between clusters is greater than $\frac{1}{2 q}$.

Proof. Let us estimate the distance between points $\mathbf{s}_{2}^{\prime}=\mathbf{0}$ of two different clusters. Indeed, $\mathbf{s}-\frac{p}{q} \mathbf{s}_{2}^{\prime \prime} \neq \mathbf{0}$, since $p \mathbf{s}_{2}^{\prime \prime}$, see (208), is not a multiple of $q$. Therefore, $\left|\left(\mathbf{s}-\frac{p}{q} \mathbf{s}_{2}^{\prime \prime}\right)_{j}\right| \geq \frac{1}{q}$, $j=1,2$. Considering that $0 \leq\left(\mathbf{s}_{2}^{\prime \prime}\right)_{j}<q, j=1,2$, we obtain that the distance between two points where $\mathbf{s}_{2}^{\prime}=\mathbf{0}$ is greater than $\frac{1}{q}-\left|\epsilon_{q}\right| q$, that is greater than $\frac{15}{16 q}$. The size of each cluster is obviously less than $\left|\epsilon_{q}\right| q\left(4 k^{r_{1}} q^{-1}+1\right) \leq \frac{1}{8 q}$. Thus, two clusters cannot overlap, the distance between them being greater than $\frac{1}{2 q}$.

We need two more properties of the lattice $\vec{p}_{\mathbf{m}},\left\|\left|\vec{p}_{\mathbf{m}} \|\right|<2 k^{r_{1}}\right.$.

Lemma 4.6. The number of vectors $\vec{p}_{\mathbf{m}}$, satisfying the inequalities $\left\|\left|\vec{p}_{\mathbf{m}} \|\right|<2 k^{r_{1}}, p_{\mathbf{m}}<\right.$ $\left|\epsilon_{q}\right| q k^{r_{1} / 3}$, does not exceed $k^{2 r_{1} / 3}$.

Proof. Suppose vectors $\vec{p}_{\mathbf{m}}$ and $\vec{p}_{\mathbf{m}^{\prime}}$ satisfy the conditions of the lemma. Then, $\| \mid \vec{p}_{\mathbf{m}}-$ $\vec{p}_{\mathbf{m}^{\prime}}|\||<4 k^{r_{1}}$. By definition of $\epsilon_{q},(2 \pi)^{-1}\left|\vec{p}_{\mathbf{m}}-\vec{p}_{\mathbf{m}^{\prime}}\right| \geq\left|\epsilon_{q}\right| q$. Thus, the distance between the points $\vec{p}_{\mathbf{m}}, \vec{p}_{\mathbf{m}^{\prime}}$ is greater than $2 \pi\left|\epsilon_{q}\right| q$ and each point can be surrounded by the disc of the radius $\pi\left|\epsilon_{q}\right| q$, the discs being disjoint. Dividing the area of the disc of the radius $2\left|\epsilon_{q}\right| q k^{r_{1} / 3}$ (we increased radius to take into account points $\vec{p}_{\mathbf{m}}$ near the boundary of the disc $\left.p_{\mathbf{m}}<\left|\epsilon_{q}\right| q k^{r_{1} / 3}\right)$ by the area of a disc of the radius $\pi\left|\epsilon_{q}\right| q$, we obtain that the number of vectors satisfying the inequality $p_{\mathbf{m}}<\left|\epsilon_{q}\right| q k^{r_{1} / 3}$ does not exceed $k^{2 r_{1} / 3}$. 
Lemma 4.7. Suppose $q$ in the inequality (206) satisfies the estimate $q>k^{2 r_{1} / 3}$. Then, the number of vectors $\vec{p}_{\mathbf{m}},\left\|\left|\vec{p}_{\mathbf{m}} \|\right|<2 k^{r_{1}}\right.$, satisfying the inequality $p_{\mathbf{m}}<k^{-2 r_{1} / 3}$ does not exceed $2^{12} \cdot k^{2 r_{1} / 3}$.

Proof. First assume $\left|\epsilon_{q}\right|>\frac{1}{64} q^{-1} k^{-r_{1}}$. Then, dividing the area of the disc of the radius $2 k^{-2 r_{1} / 3}$ by the area of a disc of the radius $\pi\left|\epsilon_{q}\right| q>\frac{1}{32} k^{-r_{1}}$, we obtain that the number of vectors satisfying the inequality $p_{\mathbf{m}}<k^{-2 r_{1} / 3}$ does not exceed $2^{12} k^{2 r_{1} / 3}$.

Second, we consider the case $\left|\epsilon_{q}\right| \leq \frac{1}{64} q^{-1} k^{-r_{1}}$. According to Lemma 4.5, the clusters do not overlap. The distance between clusters is greater than $\frac{1}{2 q}$. Therefore, dividing the area of a disc with radius $\frac{3}{2} k^{-2 r_{1} / 3}$ by the area of a disc with radius $\frac{1}{4 q}$, the last number being smaller than $\frac{1}{4} k^{-2 r_{1} / 3}$ by the conjecture of the lemma, we obtain that the number of clusters intersecting the disc of the radius $k^{-2 r_{1} / 3}$ is less than $\left(6 k^{-2 r_{1} / 3} q\right)^{2}$. Each cluster contains less than $\left(9 k^{r_{1}} q^{-1}\right)^{2}$ points. Therefore, the total number of of vectors $\vec{p}_{\mathbf{m}},\left\|\left|\vec{p}_{\mathbf{m}} \|\right|<2 k^{r_{1}}\right.$, satisfying the inequality $p_{\mathbf{m}}<k^{-2 r_{1} / 3}$ does not exceed $\left(6 k^{-2 r_{1} / 3} q\right)^{2}$. $\left(9 k^{r_{1}} q^{-1}\right)^{2}<2^{12} \cdot k^{2 r_{1} / 3}$.

\subsubsection{Lattice Points in the Nonresonant Set}

Lemma 4.8. Let $N\left(k, r_{1}, \vec{\varkappa}_{0}, \varepsilon_{0}\right)$ be the number of points $\vec{\varkappa}_{0}+\vec{p}_{\mathbf{n}},\left\|\left|\vec{p}_{\mathbf{n}} \|\right|<k^{r_{1}}\right.$ in the $\varepsilon_{0}$-neighborhood of $\mathcal{D}_{1}\left(k^{2}\right)$, where $\varepsilon_{0}=k^{-5 \mu r_{1}}$ and $\vec{\varkappa}_{0} \in \mathbb{R}^{2}$ being fixed. Then,

$$
N\left(k, r_{1}, \vec{\varkappa}_{0}, \varepsilon_{0}\right)<1000 \cdot k^{\frac{2 r_{1}}{3}+1} .
$$

Proof. Let us consider the segment $\vec{p}_{\mathbf{n}-\mathbf{n}^{\prime}}$ between two points $\vec{\varkappa}_{0}+\vec{p}_{\mathbf{n}}$ and $\vec{\varkappa}_{0}+\vec{p}_{\mathbf{n}^{\prime}}$ in the neighborhood. Obviously, $\left\|\left|\vec{p}_{\mathbf{n}-\mathbf{n}^{\prime}} \|\right|<2 k^{r_{1}}\right.$ and $p_{\mathbf{n}-\mathbf{n}^{\prime}}>k^{-\mu r_{1}}>>\varepsilon_{0}$. This means that the direction of the segment cannot be orthogonal to the curve (in fact they are almost parallel to the curve) and each end can be assigned its own angle coordinate $\varphi_{\mathbf{n}}, \varphi_{\mathbf{n}^{\prime}}$, $\varphi_{\mathbf{n}} \neq \varphi_{\mathbf{n}^{\prime}}$. We enumerate the points $\vec{\varkappa}_{0}+\vec{p}_{\mathbf{n}}$ in the order of increasing $\varphi_{\mathbf{n}}$ and connect neighboring points by segments. First we consider the segments with the length greater or equal to $\frac{1}{64} k^{-\frac{2 r_{1}}{3}}$. Since the length of $\mathcal{D}_{1}\left(k^{2}\right)$ does not exceed $3 \pi k$, the number of such segments does not exceed $650 k^{\frac{2 r_{1}}{3}+1}$.

It remains to estimate the number of segments with the length less than $\frac{1}{64} k^{-\frac{2 r_{1}}{3}}$. First, we prove that no two segments $\vec{p}_{\mathbf{n}_{1}-\mathbf{n}_{1}^{\prime}}, \vec{p}_{\mathbf{n}_{2}-\mathbf{n}_{2}^{\prime}}$ can be equal to each other. We use concavity of the curve $\mathcal{D}_{1}\left(k^{2}\right)$ and a small size $\varepsilon_{0}$ of its neighborhood. We show that for every $\vec{p}_{\mathbf{n}_{1}-\mathbf{n}_{1}^{\prime}}$ with both ends in the neighborhood, there is a point on the curve where the tangent vector is parallel to $\vec{p}_{\mathbf{n}_{1}-\mathbf{n}_{1}^{\prime}}$. Since the tangent vector changes monotonously with $\varphi$, no two vectors $\vec{p}_{\mathbf{n}_{1}-\mathbf{n}_{1}^{\prime}}$ can have the same direction. Indeed, let us consider a segment $\vec{p}_{\mathbf{n}_{1}-\mathbf{n}_{1}^{\prime}}$. Let $(x, y)$ be local coordinates associated with $\vec{p}_{\mathbf{n}_{1}-\mathbf{n}_{1}^{\prime}}$, the beginning of the segment being at the origin and the end having the coordinates $(\tau, 0), \tau=p_{\mathbf{n}_{1}-\mathbf{n}_{1}^{\prime}}$. The curve is described by the equation $y=y(x)$. It easily follows from Lemma 3.11 that $y^{\prime}(x)=o(1)$ and the curvature $\kappa$ of the curve $\mathcal{D}_{1}\left(k^{2}\right)$ is $\frac{1}{k}(1+o(1))$ at all points of the curve. Using the 
formula $\kappa(x)=\left|y^{\prime \prime}(x)\right|\left(1+y^{\prime}(x)^{2}\right)^{-3 / 2}$, we easily obtain $y^{\prime \prime}(x)=-\frac{1}{k}(1+o(1))$. Using a Taylor formula, we get $y(\tau)=y(0)+y^{\prime}(0) \tau-\frac{1}{2 k}(1+o(1)) \tau^{2}$. Note that $|y(0)|,|y(\tau)|<2 \varepsilon_{0}$, since both ends of the segment are in the $\varepsilon_{0}$-neighborhood of the curve. Considering also that $\tau>k^{-r_{1} \mu}$ and the estimate on $\varepsilon_{0}$, we conclude: $\frac{\tau}{k}=2 y^{\prime}(0)(1+o(1))+O\left(k^{-4 r_{1} \mu}\right)$. Substituting this into the Taylor formula

$$
y^{\prime}(\tau)=y^{\prime}(0)-\frac{\tau}{k}(1+o(1))
$$

we obtain: $y^{\prime}(\tau)=-y^{\prime}(0)(1+o(1))+O\left(k^{-4 r_{1} \mu}\right)$. If $y^{\prime}(\tau)$ and $y^{\prime}(0)$ have the same sign or one of them is zero, the last relation yields $\left|y^{\prime}(\tau)\right|+\left|y^{\prime}(0)\right|=O\left(k^{-4 r_{1} \mu}\right)$. This contradicts to (210), since $\tau>k^{-r_{1} \mu}$. Therefore, $y^{\prime}(\tau)$ and $y^{\prime}(0)$ have different signs. Considering that $y^{\prime}(x)$ is continuous, we obtain that there is a point $x_{0}$ in $(0, \tau)$ such that $y^{\prime}\left(x_{0}\right)=0$. This means that the isoenergetic curve at this point is parallel to $\vec{p}_{\mathbf{n}_{1}-\mathbf{n}_{1}^{\prime}}$.

To finish the proof of the lemma we consider two cases. Suppose $q$ in the inequality (206) Satisfies the estimate $q>k^{2 r_{1} / 3}$. Then, by Lemma 4.7, the number of vectors $\vec{p}_{\mathbf{n}}$, $\left\|\left|\vec{p}_{\mathbf{n}} \|\right|<2 k^{r_{1}}\right.$, satisfying the inequality $p_{\mathbf{n}}<\frac{1}{64} k^{-2 r_{1} / 3}$ does not exceed $2^{12} \cdot k^{2 r_{1} / 3}$. Since each of them can be used only once, the total number of short segments does not exceed $2^{12} \cdot k^{2 r_{1} / 3}$.

Let $q \leq k^{2 r_{1} / 3}$. If $\left|\epsilon_{q}\right|>\frac{1}{64} q^{-1} k^{-r_{1}}$. Then, obviously, $\frac{1}{64} k^{-2 r_{1} / 3}<\left|\epsilon_{q}\right| q k^{r_{1} / 3}$. Applying Lemma 4.6, we obtain that the number of segments with the length less than $\frac{1}{64} k^{-2 r_{1} / 3}$ is less than $k^{2 r_{1} / 3}$. Since each of them can be used only once, the total number of short segments does not exceed $k^{2 r_{1} / 3}$. It remains to consider the case $q \leq k^{2 r_{1} / 3},\left|\epsilon_{q}\right| \leq$ $\frac{1}{64} q^{-1} k^{-r_{1}}$. By Lemma 4.5, clusters are well separated. Considering that the distance between clusters is greater than $\frac{1}{2 q}$ and the size of each cluster is less than $\frac{1}{8 q}$, we obtain that no more than $8 \pi q k$ clusters can intersect $\varepsilon_{0}$-neighborhood of $\mathcal{D}_{1}\left(k^{2}\right)$. The part of the curve inside the clusters has the length $L_{i n}$ which is less than the double size of a cluster $10\left|\epsilon_{q}\right| k^{r_{1}}$ (the curve is concave) multiplied by the number of clusters $8 \pi q k$, i.e., $L_{i n}<80 \pi\left|\epsilon_{q}\right| q k^{r_{1}+1}$. Next, the segments with the length less than $\frac{1}{2} k^{-2 r_{1} / 3}$ cannot connect different clusters, since the distance between clusters is greater than $\frac{1}{2 q} \geq \frac{1}{2} k^{-2 r_{1} / 3}$. Therefore, any segment of the length less than $\frac{1}{2} k^{-2 r_{1} / 3}$ is inside one cluster. If we consider the segments with the length greater than $\left|\epsilon_{q}\right| q k^{r_{1} / 3}$, then the number of such segments is less than $L_{i n} /\left|\epsilon_{q}\right| q k^{r_{1} / 3}$, i.e., it is less than $80 \pi k^{2 r_{1} / 3+1}$. By Lemma 4.6, the total number of segments of the length less than $\left|\epsilon_{q}\right| q k^{r_{1} / 3}$ is less than $k^{2 r_{1} / 3}$. Each of them can be used only once. Thus, the total number of segments is less than $300 k^{2 r_{1} / 3+1}$.

\subsubsection{Lattice Points in the Resonant Set}

Here we introduce and investigate some properties of the subsets of (205) associated with strongly resonant clusters $\mathcal{M}_{2, s t r}^{j, s}$. We remind that, by Lemma 3.20 , such cluster can contain no more than two sets $\mathcal{M}_{2}^{j, s}$. We have to consider several cases (corresponding to nontrivial $\mathcal{M}_{2}^{j}$ and trivial $\mathcal{M}_{2}^{j}$ of two types in accordance with Definition [3.15). The 
goal is to prove Lemmas 4.10, 4.12, 4.13 and 4.16. They follow from Lemmas 4.9, 4.11 and 4.15, describing properties of the isoenergetic surface in the resonant region, and Lemmas 4.5,4.7. Lemma 4.14 is preparatory for 4.15, As usual, where it does not lead to confusion, we will use identical notation for similar (but not coinciding) auxiliary objects from different cases. We believe that this way it makes easier to follow parallel proofs for all cases.

Trivial case. I. First, consider $\mathbf{q},\left\|\left|\vec{p}_{\mathbf{q}} \|\right| \leq k^{\delta}\right.$, such that no vector in $\mathcal{S}_{Q}$ is a multiple of q. We put

$$
\begin{aligned}
& \mathcal{R}_{\mathbf{q}, \text { triv }}^{1}=\mathcal{R}_{\mathbf{q}}:=\left\{\vec{\varkappa} \in \mathbb{R}^{2}: \|\left.\vec{\varkappa}\right|^{2}-k^{2} \mid<k^{-40 \mu \delta} ;\right. \\
& \| \vec{\varkappa}+\left.\vec{p}_{\mathbf{q}^{\prime}}\right|^{2}-k^{2} \mid>2 k^{-40 \mu \delta \delta} \text { for } \mathbf{q}^{\prime}=c \mathbf{q}, c \neq 0,\left\|\left|\vec{p}_{\mathbf{q}^{\prime}} \|\right| \leq k^{\delta} ;\right. \\
& \| \vec{\varkappa}+\left.\vec{p}_{\mathbf{q}^{\prime \prime}}\right|^{2}-k^{2} \mid>k^{\delta_{*}} \text { for } \mathbf{q}^{\prime \prime} \neq c \mathbf{q},\left\|\left|\vec{p}_{\mathbf{q}^{\prime \prime}} \|\right| \leq k^{\delta}\right\} .
\end{aligned}
$$

Obviously, $\mathcal{R}_{\mathbf{q}}=\mathcal{R}_{\tilde{\mathbf{q}}}$ if $\tilde{\mathbf{q}}=$ cq. We will also assume that

$$
|| \vec{\varkappa}+\left.\vec{p}_{\mathbf{q}^{\prime}}\right|^{2}-k^{2} \mid \leq k^{\delta_{*}} \text { for at least one } \mathbf{q}^{\prime}=c \mathbf{q}, c \neq 0
$$

otherwise, we just deal with a non-resonant situation. Let $P_{\mathbf{q}}:=P(\delta), P(\delta)$ being defined at the beginning of Step I (we use different notation here to make it similar to constructions below for other cases). We consider the operator $P_{\mathbf{q}}\left(H(\overrightarrow{\mathcal{\varkappa}})-\left(k^{2}+\varepsilon_{0}^{\prime}\right) I\right) P_{\mathbf{q}},\left|\varepsilon_{0}^{\prime}\right| \leq k^{-165 \mu \delta}$, and its determinant $D\left(\vec{\varkappa}, k^{2}+\varepsilon_{0}^{\prime}\right)$. Let $S_{\mathbf{q}} \subset \mathcal{R}_{\mathbf{q}}$ be the set

$$
S_{\mathbf{q}}\left(\varepsilon_{0}\right):=\left\{\vec{\varkappa} \in \mathcal{R}_{\mathbf{q}}: D\left(\vec{\varkappa}, k^{2}+\varepsilon_{0}^{\prime}\right)=0 \text { for some }\left|\varepsilon_{0}^{\prime}\right| \leq \varepsilon_{0}\right\}, \quad 0<\varepsilon_{0} \leq k^{-165 \mu \delta} .
$$

Let $\vec{\varkappa}=\left(\tau_{1}, \tau_{2}\right)$ where $\tau_{1}$ is the coordinate in the direction of $\mathbf{q}$ and $\tau_{2}$ is the coordinate in the direction orthogonal to $\mathbf{q}$. We have

Lemma 4.9. The sets $D\left(\vec{\varkappa}, k^{2}\right)=0$ and $S_{\mathbf{q}}\left(\varepsilon_{0}\right)$ have the following properties in $\mathcal{R}_{\mathbf{q}}$ :

1) The equation $D\left(\vec{\varkappa}, k^{2}\right)=0$ describes at most two curves in $\mathcal{R}_{\mathbf{q}}$ which are represented by $\tau_{2}=f_{i}\left(\tau_{1}\right)$ where $\left|f_{i}^{\prime}\right| \leq k^{\delta_{*}+\mu \delta-1}, i=1,2$.

2) The set $S_{\mathbf{q}}\left(\varepsilon_{0}\right)$ belongs to $\cup_{i=1,2} S_{i}$,

$$
S_{i}\left(k, \varepsilon_{0}\right):=\left\{\vec{\varkappa}:\left|\tau_{2}-f_{i}\left(\tau_{1}\right)\right|<2 \varepsilon_{0} k^{-1},\left|\tau_{1}\right| \leq k^{\delta_{*}+\mu \delta}\right\} .
$$

3) Every curve $\tau_{2}=f_{i}\left(\tau_{1}\right)$ contains no more than $2^{31} k^{8 \delta}$ inflection points.

4) Let $\mathbf{l}$ be a segment of a straight line,

$$
\mathbf{l}=\left\{\vec{\varkappa}=\left(\tau_{1}, \beta_{1} \tau_{1}+\beta_{2}\right), \tau_{1,0} \leq \tau_{1} \leq \tau_{1,0}+\eta\right\}, \quad\left|\tau_{1}\right|<k^{\delta_{*}+\mu \delta},
$$

such that both of its ends belong to $S_{i}\left(k, \varepsilon_{0}\right)$ and $2 \varepsilon_{0}<\eta^{2} k^{-1}, 0<\eta<k^{-41 \mu \delta}$. Then, there is an inner part $\mathbf{l}^{\prime}$ of the segment which is not in $S_{i}\left(k, \varepsilon_{0}\right)$. Moreover, there is a point $\left(\tau_{1 *}, \tau_{2 *}\right)$ in $\mathbf{l}^{\prime}$ such that $f_{i}^{\prime}\left(\tau_{1 *}\right)=\beta_{1}$, i.e., the curve and the segment have the same direction when $\tau_{1}=\tau_{1 *}$. 
Proof. Let us prove that operator $P_{\mathbf{q}} H(\vec{\varkappa}) P_{\mathbf{q}}$ has exactly one eigenvalue $\lambda_{1}(\vec{\varkappa})$ satisfying $\left|\lambda_{1}(\vec{\varkappa})-k^{2}\right|<\frac{3}{2} k^{-40 \mu \delta}$. Indeed, we surround $k^{2}$ by the circle $\tilde{C}$ of the radius $\frac{3}{2} k^{-40 \mu \delta}$ and consider the perturbation series for $\left(P_{\mathbf{q}}(H(\vec{\varkappa})-z) P_{\mathbf{q}}\right)^{-1}, z \in \tilde{C}$, with respect to the resolvent of the free operator. Considering the definitions of $\mathcal{R}_{\mathbf{q}}, \tilde{C}$ and the assumption that no vector in $\mathcal{S}_{Q}$ is a multiple of $\mathbf{q}$, we easily obtain:

$$
\left\|\left(P_{\mathbf{q}}\left(H_{0}-z\right) P_{\mathbf{q}}\right)^{-1} V\left(P_{\mathbf{q}}\left(H_{0}-z\right) P_{\mathbf{q}}\right)^{-1}\right\| \leq k^{-\delta_{*}+41 \mu \delta} .
$$

Therefore, the series converges. The convergence of the series implies that $P_{\mathbf{q}} H P_{\mathbf{q}}$ has the same number of eigenvalues inside $\tilde{C}$ as $P_{\mathbf{q}} H_{0} P_{\mathbf{q}}$. i.e. exactly one. We also have $\lambda_{1}(\vec{\varkappa})=|\vec{\varkappa}|^{2}+O\left(k^{-\delta_{*}+41 \mu \delta}\right)$ and this asymptotic formula can be differentiated. From (211) it follows that $\lambda_{1}(\vec{\varkappa})=k^{2}+\varepsilon_{0}^{\prime},\left|\varepsilon_{0}^{\prime}\right|<\frac{3}{2} k^{-40 \mu \delta}$.

Next, since ||$\vec{\varkappa}+\left.\vec{p}_{\mathbf{q}^{\prime}}\right|^{2}-k^{2} \mid \leq k^{\delta_{*}}$ for at least one $\mathbf{q}^{\prime} \neq \mathbf{0}$ parallel to $\mathbf{q}$, we obtain that $\left|\frac{\partial \lambda_{1}}{\partial \tau_{1}}\right|=O\left(k^{\delta_{*}+\delta}\right)$ and $\frac{\partial \lambda_{1}}{\partial \tau_{2}}=2 \tau_{2}(1+o(1))$. This gives us the first and the second statements of the lemma (for more details see the proof of Lemma 4.9 in [1]). The third statement can be proven in complete analogy with the proof of the similar statement from Lemma 4.9 from [1].

To prove the fourth statement we introduce operators $\hat{H}_{0}$ and $E_{0}$ such that $\left(\hat{H}_{0}\right)_{\mathbf{n n}}=$ $\left(H_{0}\right)_{\mathbf{n n}}$ for $\mathbf{n} \neq \mathbf{0}$ and $\left(\hat{H}_{0}\right)_{\mathbf{0} \mathbf{0}}=1 ;\left(E_{0}\right)_{\mathbf{n n}}=0$ for $\mathbf{n} \neq \mathbf{0}$ and $\left(E_{0}\right)_{\mathbf{0} \mathbf{0}}=1$. Obviously, $\hat{H}_{0}=H_{0}\left(P_{\mathbf{q}}-E_{0}\right)+E_{0}$. We will use this operator to "normalize" the determinant $D$. Indeed, let

$$
\hat{D}\left(\vec{\varkappa}, k^{2}\right):=\frac{\operatorname{det}\left(P_{\mathbf{q}}\left(H(\vec{\varkappa})-k^{2} I\right) P_{\mathbf{q}}\right)}{\operatorname{det}\left(P_{\mathbf{q}}\left(\hat{H}_{0}(\vec{\varkappa})-k^{2}\left(I-E_{0}\right)\right) P_{\mathbf{q}}\right)} .
$$

Obviously, $D=0$ in $\mathcal{R}_{\mathbf{q}}$ if and only if $\hat{D}=0$. We consider $\vec{\varkappa}$ being in the segment $\mathbf{l}$. It follows from the estimate for $f_{i}^{\prime}$ that $\beta_{1}=O\left(k^{\delta_{*}+\mu \delta-1}\right)$ and $\left|\beta_{2}\right|=k(1+o(1))$. Let $D_{0}:=\hat{D}$ for $V=0$ :

$$
D_{0}:=\frac{\operatorname{det}\left(P_{\mathbf{q}}\left(H_{0}(\vec{\varkappa})-k^{2} I\right) P_{\mathbf{q}}\right)}{\operatorname{det}\left(P_{\mathbf{q}}\left(\hat{H}_{0}(\vec{\varkappa})-k^{2}\left(I-E_{0}\right)\right) P_{\mathbf{q}}\right)}=\operatorname{det}\left(\hat{H}_{0}(\vec{\varkappa})-k^{2} I\right) E_{0} .
$$

We easily see:

$$
D_{0}=|\vec{\imath}|^{2}-k^{2}=\tau_{1}^{2}+\left(\beta_{1} \tau_{1}+\beta_{2}\right)^{2}-k^{2} .
$$

Clearly, $D_{0}$ has no more than two roots $\tau_{1}$. Assume these roots are separated by the distance less than $2 k^{-41 \mu \delta}$ (in another case the proof is analogous, but simpler). We consider their $k^{-41 \mu \delta}$-neighborhood which we denote by $T$ (in the case of separated roots, the size of the neighborhood is $k^{-82 \mu \delta} d^{-1}, d$ being the distance between the roots). Obviously,

$$
\left|D_{0}\right|>k^{-83 \mu \delta} \text { on } \partial T \text {. }
$$


If $\tau_{1} \in T$, then the point $\left(\tau_{1}, \beta_{1} \tau_{1}+\beta_{2}\right)$ satisfy the inequalities in (211). A simple computation yields:

$$
\frac{\hat{D}}{D_{0}}=\operatorname{det}(I+B), \quad B=\left(\left(|\vec{\varkappa}|^{2}-k^{2}\right) E_{0}+I-E_{0}\right)^{-1} A
$$

where $A:=\left(P_{\mathbf{q}}\left(\hat{H}_{0}-k^{2}\left(I-E_{0}\right)\right) P_{\mathbf{q}}\right)^{-1 / 2} V\left(P_{\mathbf{q}}\left(\hat{H}_{0}-k^{2}\left(I-E_{0}\right)\right) P_{\mathbf{q}}\right)^{-1 / 2}$, the square root being chosen arbitrary. It is easy to see (cf. (215) ) that

$$
\|A\| \leq k^{-\delta_{*} / 2+21 \mu \delta} .
$$

Using (217), we obtain: $\|B\|_{1}<k^{-\delta_{*} / 2+105 \mu \delta}$. By the inequality $\|\operatorname{det}(I+B)-1\|<$ $\|B\|_{1} e^{2+\|B\|_{1}}, B$ being in the trace class, we easily obtain:

$$
\hat{D}=D_{0}\left(1+O\left(k^{-\delta_{*} / 4}\right)\right) \text { on } \partial T \text {. }
$$

By Rouché's Theorem $\hat{D}$ has the same number of zeros in $T$ as $D_{0}$ (and they are in $\frac{1}{2} k^{-41 \mu \delta}$-neighborhood of the zeros of $D_{0}$ ). Therefore, $\hat{D}$ can be represented in the form

$$
\hat{D}\left(\tau_{1}, \beta_{1} \tau_{1}+\beta_{2}\right)=\tilde{f}\left(\tau_{1}\right) \prod_{j=1}^{2}\left(\tau_{1}-\tau_{1}^{(j)}\right), \tau_{1} \in T .
$$

From (217), (219) and the minimum principle in $T$ it follows that $|\tilde{f}|>k^{-2 \mu \delta}$ when $\tau_{1} \in T$.

Let us consider the segment $\mathbf{1}$. By (220), there is a point $\left(\tau_{1}^{\prime}, \beta_{1} \tau_{1}^{\prime}+\beta_{2}\right) \in \mathbf{l}$ where

$$
|\hat{D}|>k^{-2 \mu \delta} \eta^{2}
$$

Obviously, $\left(D_{0}\right)_{\tau_{2}}=2 \tau_{2}$. Using the estimates similar to (218), we obtain $\left|\hat{D}_{\tau_{2}}^{\prime}\right| \leq 3 k$. Since by the definition of the curve $\hat{D}\left(\left(\tau_{1}^{\prime}, f_{i}\left(\tau_{1}^{\prime}\right)\right), k^{2}\right)=0$, we have

$$
\left|f_{i}\left(\tau_{1}^{\prime}\right)-\left(\beta_{1} \tau_{1}^{\prime}+\beta_{2}\right)\right|>\frac{1}{3} k^{-2 \mu \delta-1} \eta^{2} .
$$

Since $2 \varepsilon_{0} / k<\frac{1}{3} k^{-2 \mu \delta-1} \eta^{2}$, there are points in $\mathbf{l}$ which are outside $S_{i}$. At one of these points the function $\left|f_{i}\left(\tau_{1}^{\prime}\right)-\left(\beta_{1} \tau_{1}^{\prime}+\beta_{2}\right)\right|$ attains its maximum value. At this point the curve and the segment are parallel.

Let $N_{\mathbf{q}}^{(1)}\left(k, r_{1}, \vec{\varkappa}_{0}, \varepsilon_{0}\right)$ be the number of points $\vec{\varkappa}_{0}+\vec{p}_{\mathbf{n}},\left\|\left|\vec{p}_{\mathbf{n}} \|\right|<k^{r_{1}}\right.$ in $S_{\mathbf{q}}\left(k, \varepsilon_{0}\right), \vec{\varkappa}_{0}$ being fixed.

Lemma 4.10. Let $\delta_{*}<r_{1}<\infty$. If $\varepsilon_{0}<k^{-16 \mu r_{1}}$ then the number of points $N_{\mathbf{q}}^{(1)}\left(k, r_{1}, \vec{\varkappa}_{0}, \varepsilon_{0}\right)$ admits the estimate

$$
N_{\mathbf{q}}^{(1)}\left(k, r_{1}, \vec{\varkappa}_{0}, \varepsilon_{0}\right) \leq 2^{44} k^{2 r_{1} / 3+8 \delta_{*}}
$$


Proof. The proof of the lemma is analogous to that of Lemma 4.10 from [1].

Now, let

$$
\begin{aligned}
& \tilde{\mathcal{R}}_{\mathbf{q}, \text { triv }}^{1}=\tilde{\mathcal{R}}_{\mathbf{q}}:=\left\{\vec{\varkappa} \in \mathbb{R}^{2}: \|\left.\vec{\varkappa}\right|^{2}-k^{2} \mid<k^{-40 \mu \delta} ;\right. \\
& \| \vec{\varkappa}+\left.\vec{p}_{\tilde{\mathbf{q}}}\right|^{2}-k^{2} \mid<2 k^{-40 \mu \delta} \text { for one } \tilde{\mathbf{q}}=\tilde{c} \mathbf{q}, \tilde{c} \neq 0,\left\|\left|\vec{p}_{\tilde{\mathbf{q}}} \|\right| \leq k^{\delta} ;\right. \\
& \| \vec{\varkappa}+\left.\vec{p}_{\mathbf{q}^{\prime}}\right|^{2}-k^{2} \mid>4 k^{-40 \mu \delta} \text { for } \mathbf{q}^{\prime}=c \mathbf{q}, \quad c \neq 0, \tilde{c},\left\|\mid \vec{p}_{\mathbf{q}^{\prime}}\right\| \leq k^{\delta} ; \\
& \| \vec{\varkappa}+\left.\vec{p}_{\mathbf{q}^{\prime \prime}}\right|^{2}-k^{2} \mid>k^{\delta *} \text { for } \mathbf{q}^{\prime \prime} \neq c \mathbf{q},\left\|\left|\vec{p}_{\mathbf{q}^{\prime \prime}} \|\right| \leq k^{\delta}\right\} .
\end{aligned}
$$

Obviously, $\tilde{\mathcal{R}}_{\mathbf{q}, t r i v}^{1}=\tilde{\mathcal{R}}_{\tilde{\mathbf{q}}, t r i v}^{1}$. So, one can assume $\tilde{c}=1$, i.e. $\tilde{\mathbf{q}}=\mathbf{q}$. Let $\tilde{P}_{\mathbf{q}}$ be the projector onto the set

$$
\left\{\mathbf{n}:\left\|\vec{p}_{\mathbf{n}}\right\| \mid \leq k^{\delta} \text { or }\left\|\vec{p}_{\mathbf{n}-\tilde{\mathbf{q}}}\right\| \mid \leq k^{\delta}\right\} .
$$

We consider the operator $\tilde{P}_{\mathbf{q}}\left(H(\vec{\varkappa})-\left(k^{2}+\varepsilon_{0}^{\prime}\right) I\right) \tilde{P}_{\mathbf{q}}$ and its determinant $\tilde{D}\left(\vec{\varkappa}, k^{2}+\varepsilon_{0}^{\prime}\right)$, $\left|\varepsilon_{0}^{\prime}\right| \leq k^{-165 \mu \delta}$. Let $\tilde{S}_{\mathbf{q}} \subset \tilde{\mathcal{R}}_{\mathbf{q}}$ be the set

$$
\tilde{S}_{\mathbf{q}}:=\left\{\vec{\varkappa} \in \tilde{\mathcal{R}}_{\mathbf{q}}: \tilde{D}\left(\vec{\varkappa}, k^{2}+\varepsilon_{0}^{\prime}\right)=0 \text { for some }\left|\varepsilon_{0}^{\prime}\right| \leq \varepsilon_{0}\right\}, \quad 0<\varepsilon_{0} \leq k^{-165 \mu \delta} .
$$

The following lemma describes properties of $\tilde{S}_{\mathbf{q}}$.

Lemma 4.11. The sets $\tilde{D}\left(\overrightarrow{\mathcal{L}}, k^{2}\right)=0$ and $\tilde{S}_{\mathbf{q}}$ have the following properties in $\tilde{\mathcal{R}}_{\mathbf{q}}$ :

1) The equation $\tilde{D}\left(\overrightarrow{\mathcal{\varkappa}}, k^{2}\right)=0$ describes at most four curves in $\tilde{\mathcal{R}}_{\mathbf{q}}$ which are represented by $\tau_{2}=f_{i}\left(\tau_{1}\right)$ where $\vec{\varkappa}=\left(\tau_{1}, \tau_{2}\right)$ and $\left|f_{i}^{\prime}\right| \leq k^{\delta_{*}+\mu \delta-1}, i=1,2,3,4$.

2) The set $\tilde{S}_{\mathbf{q}}$ belongs to $\cup_{i} S_{i}$,

$$
S_{i}\left(k, \varepsilon_{0}\right):=\left\{\vec{\varkappa}:\left|\tau_{2}-f_{i}\left(\tau_{1}\right)\right|<2 \varepsilon_{0} k^{-1},\left|\tau_{1}\right| \leq k^{\delta_{*}+\mu \delta}\right\} .
$$

3) Every curve $\tau_{2}=f_{i}\left(\tau_{1}\right)$ contains no more than $2^{31} k^{8 \delta}$ inflection points.

4) Let $\mathbf{l}$ be a segment of a straight line,

$$
\mathbf{l}=\left\{\vec{\varkappa}=\left(\tau_{1}, \beta_{1} \tau_{1}+\beta_{2}\right), \tau_{1,0}<\tau_{1}<\tau_{1,0}+\eta\right\}, \quad\left|\tau_{1}\right|<k^{\delta_{*}+\mu \delta},
$$

such that both its ends belong to $S_{i}\left(k, \varepsilon_{0}\right), 2 \varepsilon_{0}<\eta^{4} k^{-1}, 0<\eta<k^{-41 \mu \delta}$. Then, there is an inner part $\mathbf{l}^{\prime}$ of the segment which is not in $S_{i}\left(k, \varepsilon_{0}\right)$. Moreover, there is a point $\left(\tau_{1 *}, \tau_{2 *}\right)$ in $\mathbf{l}^{\prime}$ such that $f_{i}^{\prime}\left(\tau_{1 *}\right)=\beta_{1}$, i.e., the curve and the segment have the same direction when $\tau_{1}=\tau_{1 *}$.

Proof. The proof repeats the arguments of that from Lemma 4.9 with obvious changes (two blocks instead of just one), since no vector in $S_{Q}$ is a multiple of $\mathbf{q}$.

Let $\tilde{N}_{\mathbf{q}}^{(1)}\left(k, r_{1}, \vec{\varkappa}_{0}, \varepsilon_{0}\right)$ be the number of points $\vec{\varkappa}_{0}+\vec{p}_{\mathbf{n}},\left\|\left|\vec{p}_{\mathbf{n}} \|\right|<k^{r_{1}}\right.$ in $\tilde{S}_{\mathbf{q}}\left(k, \varepsilon_{0}\right), \vec{\varkappa}_{0}$ being fixed. The analogue of Lemma 4.10 holds.

Lemma 4.12. Let $\delta_{*}<r_{1}<\infty$. If $0<\varepsilon_{0}<k^{-16 \mu r_{1}}$, then the number of points $\tilde{N}_{\mathbf{q}}^{(1)}\left(k, r_{1}, \vec{\varkappa}_{0}, \varepsilon_{0}\right)$ admits the estimate

$$
\tilde{N}_{\mathbf{q}}^{(1)}\left(k, r_{1}, \vec{\varkappa}_{0}, \varepsilon_{0}\right) \leq 2^{44} k^{2 r_{1} / 3+8 \delta_{*}} .
$$


Trivial case. II. Now we consider the case when $\mathbf{q} \in \mathcal{S}_{Q}$ but $\left|k^{2}-\left(t_{\mathbf{q}}^{\perp}\right)^{2}\right|>\frac{1}{8} k^{\delta_{*}}$, where $t_{\mathbf{q}}^{\perp}=\left(\vec{\varkappa}, \vec{\nu}_{\mathbf{q}}^{\perp}\right), \vec{\nu}_{\mathbf{q}}^{\perp}$ beind a unit vector orthogonal to $\vec{p}_{\mathbf{q}}$, see Definition 3.15, More precisely, we put

$$
\begin{aligned}
& \mathcal{R}_{\mathbf{q}, \text { triv }}^{2}=\mathcal{R}_{\mathbf{q}}:=\left\{\vec{\varkappa} \in \mathbb{R}^{2}: \|\left.\vec{\varkappa}\right|^{2}-k^{2}\left|<k^{-40 \mu \delta} ;\right| k^{2}-\left(\vec{\varkappa}, \vec{\nu}_{\mathbf{q}}^{\perp}\right)^{2} \mid>\frac{1}{8} k^{\delta_{*}} ;\right. \\
& \| \vec{\varkappa}+\left.\vec{p}_{\mathbf{q}^{\prime}}\right|^{2}-k^{2} \mid>2 k^{-40 \mu \delta} \text { for } \mathbf{q}^{\prime}=c \mathbf{q}, c \neq 0,\left\|\left|\vec{p}_{\mathbf{q}^{\prime}} \|\right| \leq k^{\delta} ;\right. \\
& \| \vec{\varkappa}+\left.\vec{p}_{\mathbf{q}^{\prime \prime}}\right|^{2}-k^{2} \mid>k^{\delta_{*}} \text { for } \mathbf{q}^{\prime \prime} \neq c \mathbf{q},\left\|\left|\vec{p}_{\mathbf{q}^{\prime \prime}} \|\right| \leq k^{\delta}\right\}
\end{aligned}
$$

and

$$
\begin{aligned}
& \tilde{\mathcal{R}}_{\mathbf{q}, \text { triv }}^{2}=\tilde{\mathcal{R}}_{\mathbf{q}}:=\left\{\vec{\varkappa} \in \mathbb{R}^{2}: \|\left.\vec{\varkappa}\right|^{2}-k^{2}\left|<k^{-40 \mu \delta} ;\right| k^{2}-\left(\vec{\varkappa}, \vec{\nu}_{\mathbf{q}}^{\perp}\right)^{2} \mid>\frac{1}{8} k^{\delta_{*}} ;\right. \\
& \| \vec{\varkappa}+\left.\vec{p}_{\tilde{\mathbf{q}}}\right|^{2}-k^{2} \mid<2 k^{-40 \mu \delta} \text { for a certain } \tilde{\mathbf{q}}=\tilde{c} \mathbf{q}, \quad \tilde{c} \neq 0,\left\|\left|\vec{p}_{\tilde{\mathbf{q}}} \|\right| \leq k^{\delta} ;\right. \\
& \| \vec{\varkappa}+\left.\vec{p}_{\mathbf{q}^{\prime}}\right|^{2}-k^{2} \mid>4 k^{-40 \mu \delta} \text { for } \mathbf{q}^{\prime}=c \mathbf{q}, c \neq 0, \tilde{c},\left\|\left|\vec{p}_{\mathbf{q}^{\prime}} \|\right| \leq k^{\delta} ;\right. \\
& \| \vec{\varkappa}+\left.\vec{p}_{\mathbf{q}^{\prime \prime}}\right|^{2}-k^{2} \mid>k^{\delta_{*}} \text { for } \mathbf{q}^{\prime \prime} \neq c \mathbf{q},\left\|\left|\vec{p}_{\mathbf{q}^{\prime \prime}} \|\right| \leq k^{\delta}\right\} .
\end{aligned}
$$

Here, one cannot assume that $\tilde{c}=1$ (unlike the trivial case I) since $\mathbf{q} \in \mathcal{S}_{Q}$ does not imply $\tilde{\mathbf{q}} \in \mathcal{S}_{Q}$ and thus $\tilde{\mathcal{R}}_{\mathbf{q}, \text { triv }}^{2} \neq \tilde{\mathcal{R}}_{\tilde{\mathbf{q}} \text {, triv }}^{2}$ in general; but we can and will assume that $\mathbf{q}$ is the generating vector of the corresponding direction. We introduce $P_{\mathbf{q}}, D, \tilde{P}_{\mathbf{q}}, \tilde{D}$ as before. Lemmas 4.94 .12 hold with the same proofs as above. The only difference is the proof of the estimates similar to (215) and (218) providing the convergence of perturbation series. Here, we provide the details which are identical for $\mathcal{R}_{\mathbf{q}}$ and $\tilde{\mathcal{R}}_{\mathbf{q}}$, so we use $\mathcal{R}_{\mathbf{q}}$ for definiteness. We check that

$$
\left|\left(\left(P_{\mathbf{q}}\left(H_{0}-z\right) P_{\mathbf{q}}\right)^{-1} V\left(P_{\mathbf{q}}\left(H_{0}-z\right) P_{\mathbf{q}}\right)^{-1}\right)_{\mathbf{n n}^{\prime}}\right| \leq k^{-\delta_{*} / 2+42 \mu \delta} .
$$

If $\mathbf{n}$ or $\mathbf{n}^{\prime}$ is not parallel to $\mathbf{q}$ the estimate is obvious. Assume that $\mathbf{n}=c \mathbf{q}$ and $\mathbf{n}^{\prime}=c^{\prime} \mathbf{q}$. The left hand side of (228) differs from zero only if $\mathbf{n}-\mathbf{n}^{\prime} \in \mathcal{S}_{Q}$. Thus, $c-c^{\prime} \in \mathbb{Z}$ and $\left|c-c^{\prime}\right| \leq Q$. In this case, considering as in the proof of (128) and using the second inequality in (226), we obtain:

$$
|| \vec{\varkappa}+\left.\vec{p}_{\mathbf{n}}\right|_{\mathbb{R}} ^{2}-k^{2}|+|\left|\vec{\varkappa}+\vec{p}_{\mathbf{n}^{\prime}}\right|_{\mathbb{R}}^{2}-k^{2} \mid>k^{\delta_{*} / 2-\delta},
$$

which proves (228). The estimate similar to (218) can be proven in the same way.

Let $N_{\mathbf{q}}^{(2)}\left(k, r_{1}, \vec{\varkappa}_{0}, \varepsilon_{0}\right)$ be the number of points $\vec{\varkappa}_{0}+\vec{p}_{\mathbf{n}},\left\|\left|\vec{p}_{\mathbf{n}} \|\right|<k^{r_{1}}\right.$ in $S_{\mathbf{q}}\left(k, \varepsilon_{0}\right)$ or $\tilde{S}_{\mathbf{q}}\left(k, \varepsilon_{0}\right), \vec{\varkappa}_{0}$ being fixed. The analogue of Lemma 4.10 holds.

Lemma 4.13. Let $\delta_{*}<r_{1}<\infty$. If $0<\varepsilon_{0}<k^{-16 \mu r_{1}}$, then the number of points $N_{\mathbf{q}}^{(2)}\left(k, r_{1}, \vec{\varkappa}_{0}, \varepsilon_{0}\right)$ admits the estimate

$$
N_{\mathbf{q}}^{(2)}\left(k, r_{1}, \vec{\varkappa}_{0}, \varepsilon_{0}\right) \leq 2^{44} k^{2 r_{1} / 3+8 \delta_{*}} .
$$


Nontrivial case. Let $\mathbf{q} \in \mathcal{S}_{Q}$ be one of two generating vectors of a direction. We put

$$
\mathcal{R}_{\mathbf{q}, \text { nontriv }}=\mathcal{R}_{\mathbf{q}}:=\left\{\vec{\varkappa} \in \mathbb{R}^{2}:\left.|| \vec{\varkappa}\right|^{2}-k^{2}\left|\leq k^{\delta_{*}}, \quad\right| k^{2}-\left(\vec{\varkappa}, \vec{\nu}_{\mathbf{q}}^{\perp}\right)^{2} \mid \leq \frac{1}{8} k^{\delta_{*}}\right\},
$$

and introduce coordinates $\tau_{1}, \tau_{2}$ as before. As in the previous cases, we will describe here properties of $S_{\mathbf{q}}\left(k, \varepsilon_{0}\right)$. However, prior considering $P_{\mathbf{q}} H P_{\mathbf{q}}$, we consider $P_{*} H P_{*}$, where $P_{*}$ is a smaller projection than $P_{\mathbf{q}}$. The projection $P_{*}$ has a property, that $P_{*} H P_{*}$ admits a separation of variables in the direction of $\mathbf{q}$ and its orthogonal, thus $\left(P_{*} V P_{*}\right)_{\mathbf{l j}}=0$, when $\mathbf{l}-\mathbf{j} \neq c \mathbf{q}$. Properties of $P_{*} H P_{*}$ can be described in terms of Schrödinger operator in dimension one. This operator is just periodic, because of condition 2 on potential $V$, see (2) and below. After investigating properties of $P_{*} H P_{*}$, we use perturbative arguments to prove that $P_{\mathbf{q}} H P_{\mathbf{q}}$ has analogous properties. This is the main place in the paper, where condition 2 on the potential is needed, since it allows to use well-known properties of periodic Schrödinger operator in dimension one, instead of a quasi-periodic one.

We consider $\vec{\varkappa} \in \mathcal{R}_{\mathbf{q}}$ and the set:

$$
\mathcal{M}_{*}(\vec{\varkappa})=\left\{n \mathbf{q}, n \in \mathbb{Z}:\left|\tau_{1}+n p_{\mathbf{q}}\right|^{2} \leq \frac{9}{8} k^{\delta_{*}}\right\} .
$$

This definition is independent on $\tau_{2}$ and piece-wise constant in $\tau_{1}$ with maybe one or two steps for every interval of $\tau_{1}$ of the size $\pi p_{\mathbf{q}} / 4$. In what follows we proceed with the constructions locally, in the intervals of $\tau_{1}$ of the size $\pi p_{\mathbf{q}} / 4$, all the statements and estimates being uniform with respect to the position of such intervals. Without the loss of generality we further assume that $\mathcal{M}_{*}$ does not depend on $\vec{\varkappa}$ in the given local interval of $\tau_{1} 2$ We consider $P_{*} H P_{*}$, where $P_{*}$ is the projector, corresponding to $\mathcal{M}_{*}(\vec{\varkappa}):\left(P_{*}\right)_{\mathbf{m m}}=1$ iff $\mathbf{m} \in \mathcal{M}_{*}$. The operator $P_{*} H P_{*}$ admits the separation of variables in the direction of $\mathbf{q}$ and its orthogonal. As usual (see (83) and below), by $\tilde{H}_{p e r}$ we will denote the corresponding one-dimensional periodic Schrödinger operator; $\tilde{\lambda}_{i}^{\text {per }}$ being its eigenvalues.

As before, we introduce the operator $P_{*}\left(H(\vec{\varkappa})-k^{2}-\varepsilon_{0}^{\prime}\right) P_{*}$, its determinant $D_{\text {aux }}\left(\vec{\varkappa}, k^{2}+\right.$ $\left.\varepsilon_{0}^{\prime}\right)$ and corresponding set $S_{\mathbf{q}}\left(\varepsilon_{0}\right) \subset \mathcal{R}_{\mathbf{q}}($ see $(213))$. 3

Lemma 4.14. Let $0<\varepsilon_{0} \leq k^{-165 \mu \delta}$. The set $D_{\text {aux }}\left(\vec{\varkappa}, k^{2}\right)=0$ and $S_{\mathbf{q}}$ have the following properties in $\mathcal{R}_{\mathbf{q}}$ :

1) For every $\left|\varepsilon_{0}^{\prime}\right| \leq \varepsilon_{0}$ the equation $D_{\text {aux }}\left(\vec{\varkappa}, k^{2}\right)=0$ describes at most four curves in $\mathcal{R}_{\mathbf{q}}$ which are represented by $\tau_{2}=f_{i}\left(\tau_{1}\right)$ where $\vec{\varkappa}=\left(\tau_{1}, \tau_{2}\right)$ and $\left|f_{i}^{\prime}\right| \leq k^{\delta_{*}+\mu \delta-1}, i=1,2,3,4$.

2) The set $S_{\mathbf{q}}$ belongs to $\cup_{i} S_{i}$,

$$
S_{i}\left(k, \varepsilon_{0}\right):=\left\{\vec{\varkappa}:\left|\tau_{2}-f_{i}\left(\tau_{1}\right)\right|<2 \varepsilon_{0} k^{-1},\left|\tau_{1}\right| \leq k^{\delta_{*}+\mu \delta}\right\} .
$$

${ }^{2}$ We note that in fact $\mathcal{M}_{*}$ is essentially the same as a $\mathcal{M}_{2}^{j, s}(\vec{\varkappa})$, see (79). However, we prefer to use the notation $\mathcal{M}_{*}$, while working with abstract geometric objects. The precise connection between $\mathcal{M}_{*}$ and sets $\mathcal{M}_{2}^{j, s}(\vec{\varkappa})$ will be established later.

${ }^{3}$ We notice that the determinant $D_{\text {aux }}$ is periodic in $\tau_{1}$ since shift of $\vec{\varkappa}$ is properly compensated by the shift of $\mathcal{M}_{*}$. 
3) Every curve $\tau_{2}=f_{i}\left(\tau_{1}\right)$ contains no more than $2^{31} k^{8 \delta}$ inflection points.

4) Let $\mathbf{l}$ be a segment of a straight line,

$$
\mathbf{l}=\left\{\vec{\varkappa}=\left(\tau_{1}, \beta_{1} \tau_{1}+\beta_{2}\right), \tau_{1,0}<\tau_{1}<\tau_{1,0}+\eta\right\}, \quad\left|\tau_{1}\right|<k^{\delta_{*}+\mu \delta},
$$

such that both its ends belong to $S_{i}\left(k, \varepsilon_{0}\right), 2 \varepsilon_{0}<\eta^{6} k^{-1}, 0<\eta<k^{-41 \mu \delta}$. Then, there is an inner part $\mathbf{l}^{\prime}$ of the segment which is not in $S_{i}\left(k, \varepsilon_{0}\right)$. Moreover, there is a point $\left(\tau_{1 *}, \tau_{2 *}\right)$ in $\mathbf{l}^{\prime}$ such that $f_{i}^{\prime}\left(\tau_{1 *}\right)=\beta_{1}$, i.e., the curve and the segment have the same direction when $\tau_{1}=\tau_{1 *}$.

Proof. The projection $P_{*}$ is defined in such a way that variables for $P_{*} H(\vec{\varkappa}) P_{*}$ can be separated, eigenvalues being described by the formula $\lambda_{i}=\tilde{\lambda}_{i}\left(\tau_{1}\right)+\tau_{2}^{2}$. Obviously, the set $D_{\text {aux }}\left(\vec{\varkappa}, k^{2}\right)=0$ is described by isoenergetic curves $\tilde{\lambda}_{i}\left(\tau_{1}\right)+\tau_{2}^{2}=k^{2}$. The formula $\tau_{2}=f_{i}\left(\tau_{1}\right)$ and the estimate for $f_{i}^{\prime}$ easily follow, as well as the second statement of the lemma.

To prove the first statement, it remains to show that the number of curves $\tau_{2}=f_{i}\left(\tau_{1}\right)$ does not exceed two. Indeed (see (93)), all eigenvalues of $P_{*} H P_{*}$ such that $\left|\lambda_{i}-k^{2}\right| \leq \frac{1}{8} k^{\delta_{*}}$ can be approximated with the high accuracy $O\left(k^{\left.-\frac{\delta_{*}}{C(Q)}\right)^{\delta_{*} / 2}}\right)$ by the eigenvalues of $\tilde{H}_{\text {per }}$ (more precisely, by $\left.\tilde{\lambda}_{i}^{\text {per }}\left(\tau_{1}\right)+\tau_{2}^{2}\right)$. Therefore,

$$
\lambda_{i}(\vec{\varkappa})=k^{2}
$$

implies

$$
\tilde{\lambda}_{i}^{p e r}\left(\tau_{1}\right)+\tau_{2}^{2}=k^{2}+O\left(k^{-\frac{\delta_{*}}{C(Q)} k^{\delta_{*} / 2}}\right) .
$$

Note that then $\left|\tilde{\lambda}_{i}^{\text {per }}\left(\tau_{1}\right)\right|=O\left(k^{\delta_{*}}\right)$. There are no more than two eigenvalues $\tilde{\lambda}_{i}^{\text {per }}$ in the $k^{-\frac{\delta_{*}}{C(Q)} k^{\delta_{*} / 2}}$-neighborhood of $k^{2}-\tau_{2}^{2}$, and consequently, no more than two eigenvalues $\lambda_{i}$ satisfying (233). Hence, the number of the curves $\tilde{\lambda}_{i}\left(\tau_{1}\right)+\tau_{2}^{2}=k^{2}$ does not exceed two, each corresponding to two curves $\tau_{2}=f_{i}\left(\tau_{1}\right)$. The third statement can be proved in complete analogy with the proof of the similar statement from Lemma 4.9 in [1].

It remains to prove the fourth statement. Let $\tau_{2}=\beta_{1} \tau_{1}+\beta_{2}$. It follows from the estimate for $f_{i}^{\prime}$ that $\beta_{1}=O\left(k^{\delta_{*}+\mu \delta-1}\right)$ and $\left|\beta_{2}\right|=k(1+o(1))$. According to the above, to investigate $S_{i} \cap \mathbf{l}$, we have to consider the equations $\tilde{\lambda}_{i}\left(\tau_{1}\right)+\left(\beta_{1} \tau_{1}+\beta_{2}\right)^{2}=k^{2}+\varepsilon_{0}^{\prime}$ with $\left|\tilde{\lambda}_{i}\left(\tau_{1}\right)\right|=O\left(k^{\delta_{*}}\right),\left|\varepsilon_{0}^{\prime}\right|<\varepsilon_{0}$. Obviously, for each real $\tau_{1}$ the last equality can hold for no more than two eigenvalues simultaneously (let's say for $\tilde{\lambda}_{i_{0}}$ and $\tilde{\lambda}_{i_{0}+1}$ ), and for all other eigenvalues we have $\left|\tilde{\lambda}_{i}\left(\tau_{1}\right)+\left(\beta_{1} \tau_{1}+\beta_{2}\right)^{2}-k^{2}\right| \geq C p_{\mathbf{q}}\left|i-i_{0}\right|$. Effectively, the estimate from below for $D_{\text {aux }}$ will follow from the estimate for each pair of close eigenvalues.

We'll need to distinguish the case of low energies (i.e. $\left.\left|\tilde{\lambda}_{i_{0}}\left(\tau_{1}\right)\right| \leq \Lambda\right)$ and large energies (i.e. $\left|\tilde{\lambda}_{i_{0}}\left(\tau_{1}\right)\right| \geq \Lambda$ ), $\Lambda$ being a large constant (to be specified later), which depends on $V$ only. Let $\left|\tilde{\lambda}_{i_{0}}\left(\tau_{1}\right)\right| \leq \Lambda$. With a proper choice of numeration, we obtain that the corresponding $\tilde{\lambda}_{i_{0}}^{\text {per }}\left(\tau_{1}\right), \tilde{\lambda}_{i_{0}+1}^{\text {per }}\left(\tau_{1}\right)$ are analytic functions of $\tau_{1}$ in a vicinity of $\tau_{1,0}$. By [43], $\left|\left(\tilde{\lambda}_{i_{0}}^{\text {per }}\left(\tau_{1}\right)\right)^{\prime \prime}\right|+\left|\left(\tilde{\lambda}_{i_{0}}^{\text {per }}\left(\tau_{1}\right)\right)^{\prime \prime \prime}\right| \neq 0$ and, hence, it is separated from zero by a constant $c(V, \Lambda)$ : 
$\left|\left(\tilde{\lambda}_{i_{0}}^{\text {per }}\left(\tau_{1}\right)\right)^{\prime \prime}\right|+\left|\left(\tilde{\lambda}_{i_{0}}^{\text {per }}\left(\tau_{1}\right)\right)^{\prime \prime \prime}\right|>c(V, \Lambda)$ in a vicinity of $\tau_{1,0}$, the size of vicinity depending on $V$ and $\Lambda$, but not $k$. This means (we recall that $\beta_{1}=O\left(k^{-1 / 2}\right)$ ) that $\tilde{\lambda}_{i_{0}}^{\text {per }}\left(\tau_{1}\right)+\left(\beta_{1} \tau_{1}+\beta_{2}\right)^{2}-k^{2}$ has no more than three zeros in the $\sigma_{\text {small }}$-neighborhood of $\tau_{1,0}, \sigma_{\text {small }}=\sigma_{\text {small }}(V, \Lambda)$. The analogous fact holds for $\tilde{\lambda}_{i_{0}+1}^{\text {per }}$, while for all other $i$-s the analogous expressions are separated from zero by a constant. Hence, denoting by $\Sigma$ the $\sigma_{\text {small }} / 6$-neighborhood of the zeros of $\tilde{\lambda}_{i_{0}}^{\text {per }}\left(\tau_{1}\right)+\left(\beta_{1} \tau_{1}+\beta_{2}\right)^{2}-k^{2}$ and $\tilde{\lambda}_{i_{0}+1}^{\text {per }}\left(\tau_{1}\right)+\left(\beta_{1} \tau_{1}+\beta_{2}\right)^{2}-k^{2}$ we have

$$
\left.\|\left(\tilde{H}_{p e r}\left(\tau_{1}\right)+\left(\beta_{1} \tau_{1}+\beta_{2}\right)^{2}-k^{2} I\right)^{-1}\right) \|<C(V, \Lambda) \text { on } \partial \Sigma .
$$

Using perturbative arguments, we easily obtain that the analogous estimate holds for $P_{*} H P_{*}$ and the corresponding resolvent has no more than 6 poles inside. Therefore,

$$
\left.\|\left(P_{*}\left(H\left(\tau_{1}\right)+\left(\beta_{1} \tau_{1}+\beta_{2}\right)^{2}-k^{2}\right) P_{*}\right)^{-1}\right) \|<C_{1}(V, \Lambda) r^{-6}, \quad 0<r<\sigma_{\text {small }} / 6,
$$

where $r$ is the distance to the nearest pole. It follows that there is a point in the interval $\left(\tau_{1,0}, \tau_{1,0}+\eta\right)$ where

$$
\left.\|\left(P_{*}\left(H\left(\tau_{1}\right)+\left(\beta_{1} \tau_{1}+\beta_{2}\right)^{2}-k^{2}\right) P_{*}\right)^{-1}\right) \|<12^{6} C_{1}(V, \Lambda) \eta^{-6} .
$$

Therefore, $\left|\tilde{\lambda}_{i}\left(\tau_{1}\right)+\left(\beta_{1} \tau_{1}+\beta_{2}\right)^{2}-k^{2}\right|>12^{-6} C_{1}(V, \Lambda)^{-1} \eta^{6}$ for all $i$. It contradicts to the assumption $\left(\tau_{1}, \beta_{1} \tau_{1}+\tau_{2}\right) \in S_{\mathbf{q}}$, since $2 \varepsilon_{0}<\eta^{6} k^{-1}$.

Let now $\left|\tilde{\lambda}_{i_{0}}\left(\tau_{1}\right)\right| \geq \Lambda>>1$. Let us consider the expression:

$$
D_{i_{0}}:=\left(\tilde{\lambda}_{i_{0}}\left(\tau_{1}\right)+\left(\beta_{1} \tau_{1}+\beta_{2}\right)^{2}-k^{2}\right)\left(\tilde{\lambda}_{i_{0}+1}\left(\tau_{1}\right)+\left(\beta_{1} \tau_{1}+\beta_{2}\right)^{2}-k^{2}\right) .
$$

We assume here that $\left|\tilde{\lambda}_{i_{0}}-\tilde{\lambda}_{i_{0}+1}\right| \leq \frac{1}{2} p_{\mathbf{q}} \Lambda^{1 / 2}$, otherwise, only one factor is needed, and this makes arguments just simpler. Since $i_{0}$ is big enough, all other eigenvalues are at the distance greater than $p_{q} \Lambda^{1 / 2}$ from this pair. Unlike each individual factor in the r.h.s. of (237), $D_{i_{0}}$ is analytic in the neighborhood of $\tau_{1,0}$ with the radius of analyticity $\sigma_{\text {large }}=$ $\pi p_{\mathbf{q}} / 4$. If $\Lambda=\Lambda(V)$ is large enough, we can apply standard perturbative arguments to compare $D_{i_{0}}$ with the same expression for $V=0$ which we denote by $D_{i_{0}, 0}$. Indeed, $D_{i_{0}, 0}$ is the polynomial of order four with respect to $\tau_{1}$ with the main coefficient $\left(1+\beta_{1}^{2}\right)^{2}$. We consider $\sigma_{\text {large }} / 100$-neighborhood of each zero and denote the connected component(s) of these neighborhoods, intersecting the $\sigma_{\text {large }} / 2$-neighborhood of $\tau_{1,0}$, by $T_{0}$. By definition, $T_{0}$ consists of $J, 1 \leq J \leq 4$ discs. We have

$$
\left|D_{i_{0}, 0}\left(\tau_{1}\right)\right|>\left(\sigma_{\text {large }} / 100\right)^{J} \quad \text { on } \partial T_{0} .
$$

Now, we choose $\Lambda=\Lambda(V)$ sufficiently large, for details see Appendix 5. By perturbation and Rouché's Theorem, $D_{i_{0}}$ has exactly $J$ zeros in $T_{0}$ (obviously, even in twice more narrow neighborhood) and

$$
\left|D_{i_{0}}\left(\tau_{1}\right)\right|>\left(\sigma_{\text {large }} / 100\right)^{J} / 2 \quad \text { on } \partial T_{0} .
$$


(For a proof see Appendix 5.) It follows $D_{i_{0}}\left(\tau_{1}\right)=g\left(\tau_{1}\right) \prod_{j=1}^{J}\left(\tau-\tau_{j}\right)$ in $T_{0}$, where, by the minimum principle, $\left|g\left(\tau_{1}\right)\right|>2^{-5}$. This means that there is $\tau_{1} \in\left(\tau_{1,0}, \tau_{1,0}+\eta\right)$ such that $\left|D_{i_{0}}\left(\tau_{1}\right)\right|>2^{-17} \eta^{4}$ and thus $\tau_{1} \notin S_{\mathbf{q}}$. Indeed, if $\tau_{1} \in S_{\mathbf{q}}$ then, by (237), $\left|D_{i_{0}}\left(\tau_{1}\right)\right|<2 \varepsilon_{0}<\eta^{6} k^{-1}$ and we arrive at the contradiction.

Obviously, the estimate (235) (and even better one, since we have at most four poles) holds in this case too.

If $\vec{\varkappa} \in \mathcal{R}_{\mathbf{q}}$ and $\vec{\varkappa}+\vec{p}_{\mathbf{m}} \in \mathcal{R}_{\mathbf{q}}, 0<\left\|\left|\vec{p}_{\mathbf{m}} \|\right|<k^{\delta}\right.$, then $\mathbf{m}=c \mathbf{q}, c \in \mathbb{R}$. This easily follows from the definition of $\mathcal{R}_{\mathbf{q}}$. Let $S_{\mathbf{q}}\left(\varepsilon_{0}\right)$ be defined by $D_{\text {aux }}$ as in the previous lemma. We define $S_{\mathbf{q}}\left(\varepsilon_{0}\right)^{\text {simple }} \subset S_{\mathbf{q}}\left(\varepsilon_{0}\right)$ and $S_{\mathbf{q}}\left(\varepsilon_{0}\right)^{\text {double }} \subset S_{\mathbf{q}}\left(\varepsilon_{0}\right)$ as follows:

$$
\begin{gathered}
S_{\mathbf{q}}\left(\varepsilon_{0}\right)^{\text {simple }}=\left\{\vec{\varkappa} \in S_{\mathbf{q}}\left(\varepsilon_{0}\right): \vec{\varkappa}+\vec{p}_{\mathbf{m}} \notin S_{\mathbf{q}}\left(\varepsilon_{0}\right) \text { when } 0<\left\|\vec{p}_{\mathbf{m}}\right\| \mid<k^{\delta}, \mathbf{m} \notin \mathcal{M}_{*}\right\} . \\
S_{\mathbf{q}}\left(\varepsilon_{0}\right)^{\text {double }}=\left\{\vec{\varkappa} \in S_{\mathbf{q}}\left(\varepsilon_{0}\right): \vec{\varkappa}+\vec{p}_{\mathbf{m}_{0}} \in S_{\mathbf{q}}\left(\varepsilon_{0}\right) \text {, for some } \mathbf{m}_{0} \notin \mathcal{M}_{*},\right. \text { and } \\
\left.\vec{\varkappa}+\vec{p}_{\mathbf{m}} \notin S_{\mathbf{q}}\left(\varepsilon_{0}\right) \text { when } 0<\left\|\vec{p}_{\mathbf{m}}\right\|<k^{\delta}, \mathbf{m} \notin \mathcal{M}_{*}, \mathbf{m} \neq \mathbf{m}_{0}\right\} .
\end{gathered}
$$

It is easy to show that the analogous definition of $S_{\mathbf{q}}\left(\varepsilon_{0}\right)^{\text {triple }}$ gives the empty set; the proof just follows the arguments from the proof of Lemma 3.20 (we recall that we work with intervals of $\tau_{1}$ just small enough so that pairs of corresponding eigenvalues $\lambda_{i_{0}}, \lambda_{i_{0}+1}$ stay stable).

Next we define the projection $P_{\mathbf{q}}$. First, let $\vec{\varkappa} \in S_{\mathbf{q}}\left(\varepsilon_{0}\right)^{\text {simple }}$. We consider the $k^{\delta_{-}}$ neighborhood of $\mathcal{M}_{*}$. Next, if it contains any $\mathbf{m}: \vec{\varkappa}+\vec{p}_{\mathbf{m}} \in \mathcal{R}_{\mathbf{q}}$, then we attach the whole set $\mathbf{m}+\mathcal{M}_{*}$ to the neighborhood 4 Thus, we obtained the $k^{\delta}$-neighborhood with "one dimensional branches" growing out of it. We denote this set by $\tilde{\mathcal{M}}_{*}$ and call it the extended $k^{\delta}$-neighborhood of $\mathcal{M}_{*}(\vec{\varkappa})$. In the case of $\vec{\varkappa} \in S_{\mathbf{q}}\left(\varepsilon_{0}\right)^{\text {double }}$ the construction of $\tilde{\mathcal{M}}_{*}$ is analogous. Namely, we consider the union of $\tilde{\mathcal{M}}_{*}(\vec{\varkappa})$ and $\tilde{\mathcal{M}}_{*}\left(\vec{\varkappa}+\vec{p}_{\mathbf{m}_{0}}\right)$ being constructed as for the simple case. With a slight abuse of notations this union we denote by $\tilde{\mathcal{M}}_{*}(\vec{\varkappa})$ again. Let $P_{\mathbf{q}}$ be the projection, corresponding to $\tilde{\mathcal{M}}_{*}(\vec{\varkappa})$ constructed for the simple and double cases. We prove the following lemma for the case $S_{\mathbf{q}}\left(\varepsilon_{0}\right)$ double. The case $S_{\mathbf{q}}\left(\varepsilon_{0}\right)^{\text {simple }}$ is completely analogous, just simpler. By $P_{1}, P_{2}$ we denote projectors corresponding to $\mathcal{M}_{*}(\vec{\varkappa}), \mathcal{M}_{*}\left(\vec{\varkappa}+\vec{p}_{\mathbf{m}_{0}}\right)$ respectively. Let $P=P_{1}+P_{2}$ and $P^{\prime}$ be the projection corresponding to the "branches", i.e. to the union of the sets $\mathbf{m}+\mathcal{M}_{*}: \vec{\varkappa}+\vec{p}_{\mathbf{m}} \in \mathcal{R}_{\mathbf{q}}, \mathbf{m} \neq \mathbf{0}, \mathbf{m}_{0}$, and $\mathbf{m} \notin \mathcal{M}_{*}$, i.e. it corresponds to "weakly-resonant" sets $\mathbf{m}+\mathcal{M}_{*}$ (cf. with the corresponding definition for $\mathcal{M}_{2}^{j, s}$ ).

As before, we introduce the coordinates $\tau_{1}, \tau_{2}$, the operator $P_{\mathbf{q}}\left(H(\vec{\varkappa})-k^{2}-\varepsilon_{0}^{\prime}\right) P_{\mathbf{q}}$ and its determinant $D\left(\overrightarrow{\mathcal{\varkappa}}, k^{2}+\varepsilon_{0}^{\prime}\right)$. Note that as above, $P_{\mathbf{q}}, P, P^{\prime}$ are piece-wise constant with respect to $\tau_{1}$.

Lemma 4.15. Let $0<\varepsilon_{0} \leq k^{-242 \mu \delta}$. The sets $D\left(\vec{\varkappa}, k^{2}\right)=0$ and $S_{\mathbf{q}}$ have the following properties in $\mathcal{R}_{\mathbf{q}}$ :

\footnotetext{
${ }^{4}$ Note that these new points are not in $S_{\mathbf{q}}$. Indeed, suppose $\vec{\varkappa}+\vec{p}_{\mathbf{m}+n \mathbf{q}} \in S_{\mathbf{q}}, n \mathbf{q} \in \mathcal{M}_{*}\left(\vec{\varkappa}+\vec{p}_{\mathbf{m}}\right)$. Since the definition of $S_{\mathbf{q}}$ is the same for $\vec{\varkappa}+\vec{p}_{\mathbf{m}}$ and $\vec{\varkappa}+\vec{p}_{\mathbf{m}+n \mathbf{q}}$ (see footnote 3) this means that $\vec{\varkappa}+\vec{p}_{\mathbf{m}} \in S_{\mathbf{q}}$, and this is not the case by definition of $S_{\mathbf{q}}\left(\varepsilon_{0}\right)^{\text {simple }}$.
} 
1) The equation $D\left(\overrightarrow{\mathcal{\varkappa}}, k^{2}\right)=0$ describes at most eight curves in $\mathcal{R}_{\mathbf{q}}$ which are represented by $\tau_{2}=f_{i}\left(\tau_{1}\right)$ where $\overrightarrow{\mathcal{\varkappa}}=\left(\tau_{1}, \tau_{2}\right)$ and $\left|f_{i}^{\prime}\right| \leq k^{\delta_{*}+\mu \delta-1}, i=1, \ldots, 85$.

2) The set $S_{\mathbf{q}}$ belongs to $\cup_{i} S_{i}$,

$$
S_{i}\left(k, \varepsilon_{0}\right):=\left\{\vec{\varkappa}:\left|\tau_{2}-f_{i}\left(\tau_{1}\right)\right|<2 \varepsilon_{0} k^{-1},\left|\tau_{1}\right| \leq k^{\delta_{*}+\mu \delta}\right\} .
$$

3) Every curve $\tau_{2}=f_{i}\left(\tau_{1}\right)$ contains no more than $2^{31} k^{8 \delta}$ inflection points.

4) Let $\mathbf{l}$ be a segment of a straight line,

$$
\mathbf{l}=\left\{\vec{\varkappa}=\left(\tau_{1}, \beta_{1} \tau_{1}+\beta_{2}\right), \tau_{1,0}<\tau_{1}<\tau_{1,0}+\eta\right\}, \quad\left|\tau_{1}\right|<k^{\delta_{*}+\mu \delta},
$$

such that both its ends belong to $S_{i}\left(k, \varepsilon_{0}\right), 2 \varepsilon_{0}<\eta^{12} k^{-1}, 0<\eta<k^{-41 \mu \delta}$. Then, there is an inner part $\mathbf{l}^{\prime}$ of the segment which is not in $S_{i}\left(k, \varepsilon_{0}\right)$. Moreover, there is a point $\left(\tau_{1 *}, \tau_{2 *}\right)$ in $\mathbf{l}^{\prime}$ such that $f_{i}^{\prime}\left(\tau_{1 *}\right)=\beta_{1}$, i.e., the curve and the segment have the same direction when $\tau_{1}=\tau_{1 *}$.

Proof. Let us consider the operator

$$
H_{\text {aux }}:=P H P+P^{\prime} H P^{\prime}+\left(P_{\mathbf{q}}-P-P^{\prime}\right) H_{0} .
$$

By the previous lemma, the resolvent of this operator as a function of $\tau_{2}$ has poles at the points $\tau_{2}=f_{i}\left(\tau_{1}\right), i \leq 8$. Let $T_{\tau_{2}}$ be the $k^{-1-40 \mu \delta_{\text {}}}$ neighborhood of these points in the complex plane of $\tau_{2}\left(\tau_{1}\right.$ is fixed). We consider $P_{\mathbf{q}} H P_{\mathbf{q}}$ as a perturbation of $H_{\text {aux }}$. Next we construct the perturbation series. Indeed,

$$
\left(H_{a u x}-k^{2}\right)^{-1 / 2}\left(P_{\mathbf{q}}\left(H-k^{2}\right) P_{\mathbf{q}}\right)\left(H_{a u x}-k^{2}-\varepsilon_{0}^{\prime}\right)^{-1 / 2}=P_{\mathbf{q}}+A,
$$

where

$$
\begin{aligned}
& A:=\left(H_{a u x}-k^{2}\right)^{-1 / 2} W\left(H_{a u x}-k^{2}\right)^{-1 / 2}, \\
& W:=\left(P_{\mathbf{q}}-P-P^{\prime}\right) V\left(P+P^{\prime}\right)+\left(P+P^{\prime}\right) V\left(P_{\mathbf{q}}-P-P^{\prime}\right)+\left(P_{\mathbf{q}}-P-P^{\prime}\right) V\left(P_{\mathbf{q}}-P-P^{\prime}\right) .
\end{aligned}
$$

Because of separation of variables, $\tau_{2}^{2}$ can be treated as a spectral parameter. Hence,

$$
\left\|\left(H_{\text {aux }}-k^{2}\right)^{-1}\right\|=O\left(k^{40 \mu \delta}\right) \quad \text { when } \tau_{2} \in \partial T_{\tau_{2}} .
$$

Next,

$$
\left\|\left(P_{\mathbf{q}}-P-P^{\prime}\right)\left(H_{0}-k^{2}\right)^{-1}\right\| \leq c k^{-\delta_{*}},
$$

since $\left(P_{\mathbf{q}}-P-P^{\prime}\right)_{\mathbf{m m}} \neq 0$ only if $\mathbf{m} \notin \mathcal{R}_{\mathbf{q} \text {, nontriv }}$, see (230). Using the last two estimates, we obtain:

$$
\|A\| \leq k^{-\delta_{*} / 4} \quad \text { when } \quad \tau_{2} \in \partial T_{\tau_{2}} .
$$

\footnotetext{
${ }^{5}$ In fact, we can still use 4 instead of 8 since, as explained at the end of the proof of Lemma 3.20, we can have two operators in the cluster only when quasi-momentum is not close to the boundary, i.e. when we have only one eigenvalue and two zeros for $D_{\text {aux }}$ corresponding to $P_{1}$ and $P_{2}$.
} 
The last inequality, in particular, shows that the perturbation series for $\left(P_{\mathbf{q}}\left(H-k^{2}\right) P_{\mathbf{q}}\right)^{-1}$ with respect to $\left(P_{\mathbf{q}}\left(H_{\text {aux }}-k^{2}-\varepsilon_{0}^{\prime}\right) P_{\mathbf{q}}\right)^{-1}$ converges and we have (see (243) $)$

$$
\left\|\left(P_{\mathbf{q}}\left(H-k^{2}\right) P_{\mathbf{q}}\right)^{-1}\right\|=O\left(k^{40 \mu \delta}\right) \quad \text { when } \tau_{2} \in \partial T_{\tau_{2}} .
$$

The perturbative arguments used in the proof of Lemma 3.24 show that $\left(P_{\mathbf{q}}\left(H-k^{2}\right) P_{\mathbf{q}}\right)^{-1}$ has the same number of zeros in $T_{\tau_{2}}$ as $\left(H_{a u x}-k^{2}\right)^{-1}$, i.e. not more than 8. Thus, solutions of $D:=\operatorname{det}\left(P_{\mathbf{q}}\left(H-k^{2}\right) P_{\mathbf{q}}\right)$ are described by at most 8 curves $\tau_{2}=f_{i}\left(\tau_{1}\right)$. The rest of the proof of the statements 1-3 is completely the same as in Lemma 4.14.

Let us prove the fourth statement. By Lemma 4.14, the estimate (235) holds for $P=P_{1}+P_{2}$. Choosing $r=\frac{1}{2} k^{-40 \mu \delta}$, we obtain

$$
\left\|\left(P\left(H-k^{2}\right) P\right)^{-1}\right\| \leq k^{241 \mu \delta} \quad \text { when } \tau_{1} \in \partial T_{\tau_{1}} .
$$

Here, by $T_{\tau_{1}}$ we denote $\frac{1}{2} k^{-40 \mu \delta}$-neighborhood of zeros corresponding to both $P_{1} H P_{1}$ and $P_{2} H P_{2}$. The resolvent $\left(P\left(H\left(\tau_{1}\right)-k^{2}\right) P\right)^{-1}$ has no more than 12 poles in $T_{\tau_{1}}$ (all of them situated in the twice more narrow neighborhood).

By definition, the resolvent $\left(P^{\prime}\left(H-k^{2}\right) P^{\prime}\right)^{-1}$ has no poles in the $k^{-40 \mu \delta}$-neighborhood of $\tau_{1,0}$. Hence, the estimate similar to (246) holds for $\left(P^{\prime}\left(H-k^{2}\right) P^{\prime}\right)^{-1}$. Further, using the perturbative arguments as above, we obtain:

$$
\left\|\left(P_{\mathbf{q}}\left(H-k^{2}\right) P_{\mathbf{q}}\right)^{-1}\right\| \leq 2 k^{241 \mu \delta} \quad \text { when } \tau_{1} \in \partial T_{\tau_{1}},
$$

and $\left(P_{\mathbf{q}}\left(H-k^{2}\right) P_{\mathbf{q}}\right)^{-1}$ has no more than 12 poles in $T_{\tau_{1}}$. Applying the same scaling as many times before we see that

$$
\left\|\left(P_{\mathbf{q}}\left(H-k^{2}\right) P_{\mathbf{q}}\right)^{-1}\right\| \leq 2 k^{241 \mu \delta}\left(\frac{\frac{1}{2} k^{-40 \mu \delta}}{r}\right)^{12},
$$

where $0<r<\frac{1}{2} k^{-40 \mu \delta}$ is the distance to the nearest pole of the resolvent. Hence, there is a point in $\left(\tau_{1,0}, \tau_{1,0}+\eta\right)$ where the norm of the resolvent is smaller than $k^{-238 \mu \delta} \eta^{-12}$. This means that the point is not in $S_{\mathbf{q}}$.

Let $N_{\mathbf{q}, \text { nontriv }}\left(k, r_{1}, \vec{\varkappa}_{0}, \varepsilon_{0}\right)$ be the number of points $\vec{\varkappa}_{0}+\vec{p}_{\mathbf{n}},\left\|\left|\vec{p}_{\mathbf{n}} \|\right|<k^{r_{1}}\right.$ in $S_{\mathbf{q}}^{\text {single }}\left(k, \varepsilon_{0}\right)$, when $\vec{\varkappa}_{0}$ is in a $S_{\mathbf{q}}^{\text {single }}\left(k, \varepsilon_{0}\right)$, or in $S_{\mathbf{q}}^{\text {double }}\left(k, \varepsilon_{0}\right)$, when $\vec{\varkappa}_{0}$ is in a $S_{\mathbf{q}}^{\text {double }}\left(k, \varepsilon_{0}\right), \vec{\varkappa}_{0}$ being fixed. The analogue of Lemma 4.10 holds.

Lemma 4.16. Let $\delta_{*}<r_{1}<\infty$. If $0<\varepsilon_{0}<k^{-16 \mu r_{1}}$, then the number of points $N_{\mathbf{q}, \text { nontriv }}\left(k, r_{1}, \vec{\varkappa}_{0}, \varepsilon_{0}\right)$ admits the estimate

$$
N_{\mathbf{q}, \text { nontriv }}\left(k, r_{1}, \vec{\varkappa}_{0}, \varepsilon_{0}\right) \leq 2^{44} k^{2 r_{1} / 3+8 \delta_{*}} .
$$

Remark 4.17. Note that the the results of Lemmas 4.14. 4.15, and, hence, that of Lemma 4.16, are stable with respect to variations of $\mathcal{M}_{*}$ at the ends. Indeed, assume that $\mathcal{M}_{*}^{\prime} \subset \mathcal{M}_{*}$ and $\mathcal{M}_{*}^{\prime}$ contains a set similar to $\mathcal{M}_{*}$ with, say, $\frac{7}{8}$ instead of $\frac{9}{8}$ in (231). Then, Lemmas 4.14. 4.15 hold for $\mathcal{M}_{*}^{\prime}$ too, since the estimate (234) and others are stable with respect to such a perturbation of $\mathcal{M}_{*}$. The sets $S_{\mathbf{q}}\left(\varepsilon_{0}\right)$ are essentially the same for $\mathcal{M}_{*}$ and $\mathcal{M}_{*}^{\prime}$ when $\varepsilon_{0}>c k^{-\frac{\delta^{*}}{C(Q)} k^{\delta^{*} / 2}}$, see (234) . 


\subsection{Preparation for Step III - Analytic Part}

\subsubsection{Model Operator for Step III}

Let $r_{2}>r_{1}>10^{8}$. Further we use the notation:

$$
\Omega\left(r_{2}\right)=\left\{\mathbf{m}:\left\|\vec{p}_{\mathbf{m}}\right\| \mid<k^{r_{2}}\right\} .
$$

We repeat for $r_{2}$ the construction of Section 3.5.1 which was done for an arbitrary $r_{1}>2$. It is easy to see that the whole construction is monotonous with respect to $r_{1}$. Namely,

$$
\begin{gathered}
\mathcal{M}\left(\varphi_{0}, r_{1}\right) \subseteq \mathcal{M}\left(\varphi_{0}, r_{2}\right), \mathcal{M}^{\prime}\left(\varphi_{0}, r_{1}\right) \subseteq \mathcal{M}^{\prime}\left(\varphi_{0}, r_{2}\right), \\
\mathcal{M}_{1}\left(\varphi_{0}, r_{1}\right) \subseteq \mathcal{M}_{1}\left(\varphi_{0}, r_{2}\right), \quad \mathcal{M}_{2}\left(\varphi_{0}, r_{1}\right) \subseteq \mathcal{M}_{2}\left(\varphi_{0}, r_{2}\right) .
\end{gathered}
$$

Again, following the procedure in Section 3.5.1, we split $\mathcal{M}_{2}\left(\varphi_{0}, r_{2}\right)$ into components $\mathcal{M}_{2}^{j}$ and, further, into $\mathcal{M}_{2}^{j, s}$, see (79) and the text above. According to Definition 3.19, we classify the sets $\mathcal{M}_{2}^{j, s}$ as weakly resonant and strongly resonant. Let $\mathcal{M}_{2}^{\text {weak }}$ be the union of all weakly resonant sets $\mathcal{M}_{2}^{j, s}$ and, correspondingly $\mathcal{M}_{2}^{s t r}=\mathcal{M}_{2} \backslash \mathcal{M}_{2}^{\text {weak }}$. By $\mathcal{M}_{2, t w}$ we denote trivial weakly resonant points. The main difficulty at this step is in treating $\mathcal{M}_{2}^{s t r} 6$

Next, we introduce an analog of $\mathcal{M}$, see (74), for the second step. Indeed, let $\varphi_{0} \in$ $\omega^{(2)}(k, \delta, 1)$ and

$$
\mathcal{M}^{(2)}\left(\varphi_{0}, r_{2}\right):=\mathcal{M}^{(2)}\left(\varphi_{0}, r_{2}\right)=\left\{\mathbf{m} \in \mathcal{M} \backslash \mathcal{M}_{2}^{\text {weak }}: \varphi_{0} \in \mathcal{O}_{\mathbf{m}}^{(2)}\left(10 r_{1}^{\prime}, 1\right)\right\}
$$

where $\mathcal{O}_{\mathbf{m}}^{(2)}\left(10 r_{1}^{\prime}, \tau\right)$ is the union of the disks of the radius $\tau k^{-10 r_{1}^{\prime}}$ with the centers at poles of the resolvent of $k^{\delta}$-component containing $\vec{\varkappa}^{(1)}\left(\varphi_{0}\right)+\vec{p}_{\mathbf{m}}$. More precisely, for each $\mathbf{m} \in \mathcal{M}\left(\varphi_{0}, r_{2}\right) \backslash \mathcal{M}_{2}^{\text {weak }}\left(\varphi_{0}, r_{2}\right)$ we construct the $k^{\delta}$-neighborhood (in $\||\cdot| \mid$ norm) around it. Note that only points from $\mathcal{M}_{1}$ generate separated $k^{\delta}$-boxes around each of them. Points corresponding to strongly resonant sets $\mathcal{M}_{2}^{j, s}$ generate $k^{\delta}$-clusters around no more than two different strongly resonant $\mathcal{M}_{2}^{j, s}$, thus their size is $O\left(k^{\delta_{*}}\right)$ along $\mathcal{M}_{2}^{j, s}$. The details are provided in Step II (see (145)). So, when we say $k^{\delta}$-component/cluster/box we, in fact, mean one of these sets. Thus, $\mathcal{O}_{\mathbf{m}}^{(2)}\left(10 r_{1}^{\prime}, \tau\right)$ is the union of the disks of the radius $\tau k^{-10 r_{1}^{\prime}}$ with the centers at poles of the operator $\left(P(\mathbf{m})\left(H\left(\vec{\varkappa}^{(1)}(\varphi)\right)-k^{2} I\right) P(\mathbf{m})\right)^{-1}$, where $P(\mathbf{m})$ is the projection onto a particular $k^{\delta}$-component containing $\vec{\varkappa}^{(1)}\left(\varphi_{0}\right)+\vec{p}_{\mathbf{m}}$. For $\mathbf{m}$ generating the same $k^{\delta}$-component corresponding sets $\mathcal{O}_{\mathbf{m}}^{(2)}\left(10 r_{1}^{\prime}, \tau\right)$ are identical. By construction of the non-resonant set $\omega^{(2)}(k, \delta, 1)$, we have $\mathcal{M}^{(2)} \cap \Omega\left(r_{1}\right)=\emptyset$.

Further we use the property of the set $\mathcal{M}^{(2)}$ formulated in the next lemma.

Lemma 4.18. Let $\mathbf{m}_{0} \in \Omega\left(r_{2}\right), 1 / 20<\gamma^{\prime}<20$ and $\Pi_{\mathbf{m}_{0}}$ be the $k^{\gamma^{\prime} r_{1}}$-neighborhood (in $\left\||\cdot \||-\right.$ norm) of $\mathbf{m}_{0}$. Then, the set $\Pi_{\mathbf{m}_{0}}$ contains less than ck $^{2 \gamma^{\prime} r_{1} / 3+1}$ elements of $\mathcal{M}^{(2)}$.

\footnotetext{
${ }^{6}$ It follows from Definition 3.19 that each trivial weakly resonant $\mathcal{M}_{2}^{j, s}$ can be treated as points from the complement to $\mathcal{M}$ with $k^{-40 \mu \delta}$ instead of $k^{\delta_{*}}$ in (72), (744) (it does not make a lot of difference). A non-trivial weakly resonant set can be treated similarly up to technical details.
} 
Proof. If $\mathbf{m} \in \mathcal{M}^{(2)}$, then there is a $\varphi_{*}:\left|\varphi_{0}-\varphi_{*}\right|<k^{-10 r_{1}^{\prime}}$ such that

$$
\operatorname{det}\left(P(\mathbf{m})\left(H\left(\vec{\varkappa}^{(1)}\left(\varphi_{*}\right)\right)-k^{2} I\right) P(\mathbf{m})\right)=0 .
$$

Therefore, for some $\varepsilon_{0}^{\prime}:\left|\varepsilon_{0}^{\prime}\right|<\varepsilon_{0}, \varepsilon_{0}:=c k^{1-10 r_{1}^{\prime}}$,

$$
\operatorname{det}\left(P(\mathbf{m})\left(H\left(\vec{\varkappa}^{(1)}\left(\varphi_{0}\right)\right)-\left(k^{2}+\varepsilon_{0}^{\prime}\right) I\right) P(\mathbf{m})\right)=0 .
$$

Indeed, if (253) holds for no $\varepsilon^{\prime}$, then $\left\|\left(P(\mathbf{m})\left(H\left(\vec{\varkappa}^{(1)}\left(\varphi_{0}\right)\right)-k^{2} I\right) P(\mathbf{m})\right)^{-1}\right\|<c k^{-1+10 r_{1}^{\prime}}$, since $\varphi_{0}$ is real and, hence, $H\left(\vec{\varkappa}^{(1)}\left(\varphi_{0}\right)\right.$ is selfadjoint. Using Hilbert identity, we obtain that $\left(P(\mathbf{m})\left(H\left(\vec{\varkappa}^{(1)}\left(\varphi_{*}\right)\right)-k^{2} I\right) P(\mathbf{m})\right)^{-1}$ is bounded. This contradicts to (252). Hence, (253) holds for some $\varepsilon_{0}^{\prime}:\left|\varepsilon_{0}^{\prime}\right|<\varepsilon_{0}$.

Suppose $\mathbf{m} \in \mathcal{M}_{1}\left(\varphi_{0}, r_{2}\right)$. Then, (253) means that $\left|\lambda^{(1)}\left(\vec{\varkappa}^{(1)}\left(\varphi_{0}\right)+\vec{p}_{\mathbf{m}}\right)-k^{2}\right|<\varepsilon_{0}$. Introducing the notation $\vec{\varkappa}_{0}=\vec{\varkappa}^{(1)}\left(\varphi_{0}\right)+\vec{p}_{\mathbf{m}_{0}}$, we rewrite the last inequality in the form: $\left|\lambda^{(1)}\left(\vec{\varkappa}_{0}+\vec{p}_{\mathbf{m}-\mathbf{m}_{0}}\right)-k^{2}\right|<\varepsilon_{0}$, where $\left\|\left|\vec{p}_{\mathbf{m}-\mathbf{m}_{0}} \|\right|<k^{\gamma^{\prime} r_{1}}\right.$. It follows that $\vec{\varkappa}_{0}+\vec{p}_{\mathbf{m}-\mathbf{m}_{0}}$ is in the real $c \varepsilon_{0} k^{-1}$-neighborhood of $\mathcal{D}_{1}\left(k^{2}\right)$. Applying Lemma 4.8, we obtain that the number of such points does not exceed $c k^{2 \gamma^{\prime} r_{1} / 3+1}$.

Let $\mathbf{m} \in \mathcal{M}_{2}^{s t r}\left(\varphi_{0}, r_{2}\right)$. Namely, let us consider all $\mathbf{m}$ belonging to a particular component $\mathcal{M}_{2, s t r}^{j, s}\left(\varphi_{0}, r_{2}\right)$. Then, ||$\vec{\varkappa}^{(1)}\left(\varphi_{0}\right)+\left.\vec{p}_{\mathbf{m}}\right|_{\mathbb{R}} ^{2}-k^{2} \mid<k^{\delta_{*}}$ and (253) holds, $P(\mathbf{m})$ being the projection on $\tilde{\mathcal{M}}_{2, s t r}^{j, s}\left(\varphi_{0}, r_{2}\right)$. Using again the notation $\vec{\varkappa}_{0}=\vec{\varkappa}^{(1)}\left(\varphi_{0}\right)+\vec{p}_{\mathbf{m}_{0}}$, and the definition of $\mathcal{M}_{2, s t r}^{j, s}\left(\varphi_{0}, r_{2}\right)$, we see that in terms of Section 4.3.3, (253) means $\vec{\varkappa}_{0}+\vec{p}_{\mathbf{m}-\mathbf{m}_{0}} \in S_{\mathbf{q}}\left(k, \varepsilon_{0}\right)$, see (213). Applying Lemmas 4.10]4.12]4.13]4.16 and using (253), we obtain that the number of such points does not exceed $c k^{2 \gamma^{\prime} r_{1} / 3+8 \delta_{*}}$ for a fixed set $\mathcal{M}_{2, s t r}^{j, s}\left(\varphi_{0}, r_{2}\right) 7$ Since the number of such sets is bounded by $c k^{4 \delta}$, the lemma is proven. $\square$

Let us split $k^{r_{2}}$-box into $k^{\gamma r_{1}}$-boxes as described below. In the whole construction below we will have $\gamma=\frac{1}{5}$, but in some cases we will refer to the similar estimates with other values of $\gamma$. That's why in what follows we prefer to use implicit notation. The procedure consists of several steps. On each step we introduce a new scale of a box. Further structure will acquire additional scales at each step of approximation procedure. This is why we call the procedure Multiscale Construction in the Space of Momenta.

1. Simple region. Let $\Omega_{s}^{(2)}\left(r_{2}\right)$ be the collection of $\mathbf{m} \in \Omega\left(r_{2}\right)$ with small values of $p_{\mathbf{m}}$, namely, $\Omega_{s}^{(2)}\left(r_{2}\right)=\left\{\mathbf{m} \in \Omega\left(r_{2}\right): 0<p_{\mathbf{m}} \leq k^{-5 r_{1}^{\prime}}\right\}$. Then, $k^{r_{1}}$-boxes around such $\mathbf{m}$-s are similar to $\Omega\left(r_{1}\right)$, since $p_{\mathbf{m}}$ is small. Indeed, it is easy to see that $\Omega_{s}^{(2)}\left(r_{2}\right) \subset \mathcal{M}\left(\varphi_{0}, r_{2}\right)$, since $p_{\mathbf{m}}$ is small, see (74), (72). Next, if $\mathbf{m} \in \Omega_{s}^{(2)}\left(r_{2}\right)$, then there are no other elements of $\mathcal{M}\left(\varphi_{0}, r_{2}\right)$ in the $k^{\delta}$-box around $\mathbf{m}$. Indeed, let $\vec{\varkappa}=\vec{\varkappa}^{(1)}\left(\varphi_{0}\right)+\vec{p}_{\mathbf{m}}$. It is is a small perturbation of $\vec{\varkappa}^{(1)}\left(\varphi_{0}\right)$, hence it satisfies

\footnotetext{
${ }^{7}$ Note that in Lemmas 4.14, 4.15 the set $\mathcal{M}_{*}$ essentially coincides with $\mathcal{M}_{2, s t r}^{j, s}\left(\varphi_{0}, r_{2}\right)-\mathbf{m}$, see also Remark 4.17
} 
||$\vec{\varkappa}+\left.\vec{p}_{\mathbf{n}}\right|^{2}-|\vec{\varkappa}|^{2} \mid>\frac{\tau}{2} k^{1-40 \mu \delta}(1+o(1))$ when $0<\left\|\left|\vec{p}_{\mathbf{n}} \|\right|<k^{\delta}\right.$, see (63)). This means $\mathbf{m}+\mathbf{n} \notin \mathcal{M}\left(\varphi_{0}, r_{2}\right)$. Further, if $\mathbf{m} \in \Omega_{s}^{(2)}\left(r_{2}\right)$, then there are no other elements of $\Omega_{s}^{(2)}\left(r_{2}\right)$ in the surrounding box of the size $k^{r_{1}}$, see (30). Last, $\mathbf{m}$ itself can belong or do not belong to $\mathcal{M}^{(2)}$, but there are no other elements of $\mathcal{M}^{(2)}$ in the $k^{r_{1}}$-box around such $\mathbf{m}$. Indeed, $\vec{\imath}^{(1)}\left(\varphi_{0}\right)$ satisfies the conditions of Lemma 3.31. This means that the $k^{\delta}$-cluster around each q: $0<\left\|\left|\vec{p}_{\mathbf{q}} \|\right|<k^{r_{1}}\right.$ is non-resonant in the sense of (251)(253). Moreover, the $k^{\delta}$-box around each $\mathbf{m}+\mathbf{q}: 0<\left\|\left|\vec{p}_{\mathbf{q}} \|\right|<k^{r_{1}}\right.$ is non-resonant too, since $p_{\mathbf{m}}$ is sufficiently small. This means $\mathbf{m}+\mathbf{q} \notin \mathcal{N}^{(2)}\left(\varphi_{0}, r_{2}\right)$.

For each $\mathbf{m} \in \Omega_{s}^{(2)}\left(r_{2}\right)$ we consider its $k^{r_{1} / 2}$-neighborhood. The union of such boxes we call the simple region and denote it by $\Pi_{s}\left(r_{2}\right)$. The corresponding projection is $P_{s}$. Note, that the distance from the simple region to the nearest point of $\mathcal{M}^{(2)}$ is greater than $\frac{1}{2} k^{r_{1}}$.

2. Black region. Next, we split $\Omega\left(r_{2}\right) \backslash\left(\Omega\left(r_{1}\right) \cup \Pi_{s}\right)$ into boxes of the size $k^{\gamma r_{1}}$. All elements $\mathbf{m} \in \mathcal{M}^{(2)}$ there satisfy $p_{\mathbf{m}}>k^{-5 r_{1}^{\prime}}$. We call a box black, if together with its neighbors it contains more than $k^{\gamma r_{1} / 2+\delta_{0} r_{1}}$ elements of $\mathcal{M}^{(2)}, \delta_{0}=\gamma / 100$ (in particular $\delta_{0} r_{1}>100$ ). Let us consider all "black" boxes together with their $k^{\gamma r_{1}+\delta_{0} r_{1}}$-neighborhoods. We call this the black region. Note that that the size of the neighborhoods involved is much smaller than the size of the neighborhoods $k^{r_{1} / 2}$ for the simple region, since $\gamma+\delta_{0}<\frac{1}{2}$. The estimates for the size of the black region will be proven in Lemma 4.19, We denote the black region by $\Pi_{b}$. The corresponding projector is $P_{b}$. Obviously the distance between black and simple regions is greater than $\frac{1}{2} k^{r_{1}}$.

3. Grey region. By a white box we mean a $k^{\gamma r_{1}}$-box, which together with its neighbors contains no more than $k^{\gamma r_{1} / 2+\delta_{0} r_{1}}$ elements of $\mathcal{M}^{(2)}$. Every white box we split into "small" boxes of the size $k^{\gamma r_{1} / 2+2 \delta_{0} r_{1}}$. We call a small box "grey", if together with its neighbors it contains more than $k^{\gamma r_{1} / 6-\delta_{0} r_{1}}$ elements of $\mathcal{M}^{(2)}$. The grey region is the union of all grey small boxes together with their $k^{\gamma r_{1} / 2+2 \delta_{0} r_{1}}$-neighborhoods. Note that that the size of the neighborhoods involved is much smaller than the size of the neighborhoods the simple and black regions. The estimates for the size of the grey region will be proven in Lemma 4.20. The notation for this region is $\Pi_{g}$. The corresponding projector is $P_{g}$. The part of the grey region, which is outside the black region, we denote by $\Pi_{g}^{\prime}$ and the corresponding projection by $P_{g}^{\prime}$. Obviously, the distance between grey and simple regions is greater than $\frac{1}{2} k^{r_{1}}$.

4. White region. By a white small box we mean a small box, which together with its neighbors has no more than $k^{\gamma r_{1} / 6-\delta_{0} r_{1}}$ elements of $\mathcal{M}^{(2)}$. In each small white box we consider $k^{\gamma r_{1} / 6}$-boxes around each point of $\mathcal{M}^{(2)}$. The union of such $k^{\gamma r_{1} / 6}$-boxes we call the white region and denote it by $\Pi_{w}$. The corresponding projection is $P_{w}$. Note that the size of the neighborhoods involved is much smaller than the size of the 
neighborhoods the simple, black and grey regions. The estimates for the size of the white region will be proven in Lemma 4.21. The part of the white region which is outside the black and grey regions, we denote $\Pi_{w}^{\prime}$ and the corresponding projection by $P_{w}^{\prime}$. Obviously, the distance between grey and simple regions is greater than $\frac{1}{2} k^{r_{1}}$.

5. Non-resonant region. We also consider $k^{\delta}$-components surrounding points in the set $\mathcal{M}\left(r_{2}, \varphi_{0}\right) \backslash\left(\mathcal{M}\left(r_{1}, \varphi_{0}\right) \cup \mathcal{M}^{(2)} \cup \Omega_{s}^{(2)}\left(r_{2}\right) \cup \mathcal{M}_{2, t w}\left(\varphi_{0}, r_{2}\right)\right)$. The union of these components we call the non-resonant region $\Pi_{n r}$. The corresponding projection is $P_{n r}$. The part of the non-resonant region which is outside $\Pi_{s} \cup \Pi_{b} \cup \Pi_{g} \cup \Pi_{w}$, we denote $\Pi_{n r}^{\prime}$ and the corresponding projection by $P_{n r}^{\prime}$.

Let

$$
P_{r}:=P_{s}+P_{b}+P_{g}^{\prime}+P_{w}^{\prime}, \quad P^{(2)}:=P_{r}+P_{n r}^{\prime}+P\left(r_{1}\right),
$$

index $r$ standing for "resonant".

First, we establish $3 k^{\gamma r_{1}+\delta_{0} r_{1}}$-equivalence relation between black boxes. Then the set $\Pi_{b}$ can be represented as the union of components (clusters) separated by distance no less than $k^{\gamma r_{1}+\delta_{0} r_{1}}$. We denote such a component by $\Pi_{b}^{j}$.

Lemma 4.19. 1. Each $\Pi_{b}^{j}$ contains no more than $\mathrm{ck}^{\gamma r_{1} / 2-\delta_{0} r_{1}+3}$ black boxes.

2. The size of $\Pi_{b}^{j}$ in $\left\||\cdot \||\right.$ norm is less than $\mathrm{ck}^{3 \gamma r_{1} / 2+3}$.

3. Each $\Pi_{b}^{j}$ contains no more than $\mathrm{ck}^{\gamma_{1}+3}$ elements of $\mathcal{M}^{(2)}$. Moreover, any box of $\left\||\cdot \||\right.$-size $c k^{3 \gamma r_{1} / 2+3}$ containing $\Pi_{b}^{j}$ has no more than $\mathrm{ck}^{\gamma r_{1}+3}$ elements of $\mathcal{M}^{(2)}$ inside.

Proof. Let $n_{b}$ be the number of black boxes in $\Pi_{b}^{j}, L_{b}$ be the size of $\Pi_{b}^{j}$ and $N_{b}$ the number of elements of $\mathcal{M}^{(2)}$ in $\Pi_{b}^{j}$. Obviously, $L_{b}<n_{b} 3 k^{\gamma r_{1}+\delta_{0} r_{1}}$ and $N_{b}>c n_{b} k^{\gamma r_{1} / 2+\delta_{0} r_{1}}$. By Lemma 4.18, $N_{b}<c L_{b}^{2 / 3} k$. Solving the last three inequalities for $n_{b}$, we get $n_{b}<$ $c k^{\gamma r_{1} / 2-\delta_{0} r_{1}+3}$. It follows $L_{b}<c k^{3 \gamma r_{1} / 2+3}$. Next, we consider a box of the size $k^{3 \gamma r_{1} / 2+3}$, containing $\Pi_{b}^{j}$. Using again Lemma 4.18, we obtain that the number of elements of $\mathcal{M}^{(2)}$ in this box is less than $c L_{b}^{2 / 3} k$. Therefore, $N_{b}<c k^{\gamma r_{1}+3}$.

Second, we establish $3 k^{\gamma r_{1} / 2+2 \delta_{0} r_{1}}$-equivalence relation between small grey boxes. Then the set $\Pi_{g}$ can be represented as the union of components separated by distance no less than $k^{\gamma r_{1} / 2+2 \delta_{0} r_{1}}$. We denote each such component as $\Pi_{g}^{j}$.

Lemma 4.20. 1. Each $\Pi_{g}^{j}$ contains no more than $c k^{\gamma r_{1} / 3+2 \delta_{0} r_{1}}$ grey boxes.

2. The size of $\Pi_{g}^{j}$ in $\left\||\cdot \||\right.$ norm is less than ck $^{5 \gamma r_{1} / 6+4 \delta_{0} r_{1}}$.

3. Each $\Pi_{g}^{j}$ contains no more than $k^{\gamma r_{1} / 2+\delta_{0} r_{1}}$ elements of $\mathcal{M}^{(2)}$. 
Proof. Let us consider a part of $\Pi_{g}^{j}$ belonging to one "big" white box. Let $n_{g}$ be the number of grey boxes in $\Pi_{g}^{j}$. Let $L_{g}$ be the size of $\Pi_{g}^{j}$ and $N_{g}$ be the number of elements of $\mathcal{M}^{(2)}$ in $\Pi_{g}^{j}$. Obviously, $N_{g}>c n_{g} k^{\gamma r_{1} / 6-\delta_{0} r_{1}}$. By definition of a big white box $N_{g}<$ $k^{\gamma r_{1} / 2+\delta_{0} r_{1}}$. Therefore, $n_{g}<c k^{\gamma r_{1} / 3+2 \delta_{0} r_{1}}$. Clearly, $L_{g}<n_{g} 3 k^{\gamma r_{1} / 2+2 \delta_{0} r_{1}}<c k^{5 \gamma r_{1} / 6+4 \delta_{0} r_{1}}$. Since $\delta_{0}<\gamma / 24$, we obtain that the size of each grey component is much less than the size $k^{\gamma r_{1}}$ of a big box. The lemma is proven under condition that $\Pi_{g}^{j}$ is inside one of white boxes. Suppose $\Pi_{g}^{j}$ intersects more than one white box. Considering that the size of $\Pi_{g}^{j}$ in each big white box is much less than the size of this box, we conclude that $\Pi_{g}^{j}$ fits into neighboring boxes and satisfies the estimates proven above.

Third, we consider points of $\mathcal{M}^{(2)}$ in small white boxes. We establish $3 k^{\gamma r_{1} / 6}$-equivalence relation between them. Considering $k^{\gamma r_{1} / 6}$-neighborhoods of the points in $\mathcal{M}^{(2)}$, we see that this neighborhoods form clusters $\Pi_{w}^{j}$ of $\Pi_{w}$ separated by the distance no less than $k^{\gamma r_{1} / 6}$. The number of $\mathcal{M}^{(2)}$ points in a white cluster we denote by $N_{w}^{j}$.

Lemma 4.21. 1. The size of $\Pi_{w}^{j}$ in $\left\||\cdot \||\right.$ norm is less than $c k^{\gamma r_{1} / 3-\delta_{0} r_{1}}$.

2. Each $\Pi_{w}^{j}$ contains no more than $k^{\gamma r_{1} / 6-\delta_{0} r_{1}}$ points of $\mathcal{M}^{(2)}$.

Proof. Let us consider points of $\mathcal{M}^{(2)}$ in a small white box. By the definition of the white small box, the number of such points does not exceed $k^{\gamma r_{1} / 6-\delta_{0} r_{1}}$. We consider the $k^{\gamma r_{1} / 6}$-neighborhoods of these points. They can form clusters. The total contribution from all points of $\mathcal{M}^{(2)}$ in the small white box and its neighbors, obviously, does not exceed $3 k^{\gamma r_{1} / 3-\delta_{0} r_{1}}$, which is much less than the size of a small white box. Therefore, each $\Pi_{w}^{j}$ can't spread outside of the small white box and its neighbors. This proves both statements of the lemma.

Next, we slightly change definitions of the simple, black, grey and white areas to adjust their boundary to the structure of clusters. Namely (cf. construction of the $k^{\delta}$ neighborhood of strongly resonant sets $\mathcal{M}_{2}^{j, s}$ above), if $k^{\delta}$-cluster generated by points of $\mathcal{M}\left(\varphi_{0}, r_{2}\right) \backslash\left(\mathcal{M}^{(2)} \cup \mathcal{M}_{2, t w}\left(r_{2}, \varphi_{0}\right)\right)$ intersects a $k^{\delta}$-neighborhood of a simple, white, grey or black area, then we include it into the corresponding region. This "addition" does not change formulation of Lemmas 4.19, 4.20, 4.21, since the size of a $k^{\delta}$-cluster is $O\left(k^{\delta_{*}}\right)$ which is much smaller that the sizes of $\Pi_{b}^{j}, \Pi_{g}^{j}, \Pi_{w}^{j}$. If a white cluster has a distance less than $k^{\gamma r_{1} / 6}$ to a grey or black cluster, we include it into that with the lighter color. This "addition" also does not change formulation of Lemmas 4.19, 4.20, since the size of a white cluster is much smaller that the characteristic sizes of $\Pi_{b}^{j}, \Pi_{g}^{j}$. If a grey cluster has a distance less than $k^{\gamma r_{1} / 2+2 \delta_{0} r_{1}}$ to a black cluster $\Pi_{b}^{j}$, we include it into this $\Pi_{b}^{j}$. This "addition" does not change formulation of Lemma 4.19, since the size of a grey cluster is much smaller that the characteristic size of any $\Pi_{b}^{j}$. The new structure has the following properties. If the intersection of the $k^{\gamma r_{1} / 6}$-neighborhood of a white cluster with grey or black area is not empty, then this cluster is completely in this area. If the intersection of the $k^{\gamma r_{1} / 2+2 \delta_{0} r_{1}}$-neighborhood of a grey cluster with the black area is not empty, then 
this cluster is completely in this area. 8 Recall that $\delta_{0}<\gamma / 24, \gamma<1 / 3$. This means that each component of the white, grey, black and non-resonance region is much smaller in $\||\cdot| \mid$-size than $\Omega\left(r_{1}\right)$. Moreover, there are no points of $\mathcal{M}^{(2)}$ inside $\Omega\left(r_{1}\right)$. If the $k^{\gamma r_{1} / 6_{-}}$ neighborhood of a white cluster intersect $\Omega\left(r_{1}\right)$, we reduce $\Omega\left(r_{1}\right)$ by this neighborhood. This insignificant reduction does not change Step II. We make a similar reduction of $\Omega\left(r_{1}\right)$ if it is intersected by neighborhoods of grey or black clusters. Sometimes it will be convenient to numerate the projections $P\left(r_{1}\right), P_{b}, P_{g}^{\prime}, P_{w}^{\prime}, P_{n r}^{\prime}, P_{s}$ by indices $0,1,2,3,4,5$ as $P_{0}, P_{1}, P_{2}, P_{3}, P_{4}, P_{5}$. The corresponding sets are $\Pi_{i}$. Note that each $\Pi_{i}$ consists of components $\Pi_{i j}, j=1, \ldots, J(i)$ as described in the construction of sets $\Pi_{i}$. The distance between closest components $\Pi_{i j}, i=1,2,3,4,5$ with the same first index is greater than $k^{\gamma r_{1}+\delta_{0} r_{1}}, k^{\gamma r_{1} / 2+2 \delta_{0} r_{1}}, k^{\gamma r_{1} / 6}, k^{\delta}, k^{\gamma r_{1}}$, correspondingly. Then we can rewrite $P^{(2)}$, see (254), in the form:

$$
P^{(2)}=\sum_{i=0}^{5} P_{i} .
$$

We introduce the boundaries $\partial \Omega\left(r_{1}\right), \partial \Pi_{b}, \partial \Pi_{g}^{\prime}, \partial \Pi_{w}^{\prime}, \partial \Pi_{n r}^{\prime}, \partial \Pi_{s}$ of the sets $\Omega\left(r_{1}\right), \Pi_{b}$, $\Pi_{g}^{\prime}, \Pi_{w}^{\prime}, \Pi_{n r}^{\prime}, \Pi_{s}$ as follows: $\partial \Omega\left(r_{1}\right), \partial \Pi_{b}, \partial \Pi_{g}^{\prime}, \partial \Pi_{w}^{\prime}, \partial \Pi_{n r}^{\prime}, \partial \Pi_{s}$ are the sets of points in $\Omega\left(r_{1}\right), \Pi_{b}, \Pi_{g}^{\prime}, \Pi_{w}^{\prime}, \Pi_{n r}^{\prime}, \Pi_{s}$ which can be connected by $V$ with the complements of $\Omega\left(r_{1}\right)$, $\Pi_{b}, \Pi_{g}^{\prime}, \Pi_{w}^{\prime}, \Pi_{n r}^{\prime}, \Pi_{s}$, respectively. The corresponding projectors we denote as $P^{\partial}\left(r_{1}\right), P_{b}^{\partial}$, $P_{g}^{\prime \partial}, P_{w}^{\prime \partial}, P_{n r}^{\prime \partial}, P_{s}^{\partial}$ or $P_{i}^{\partial}, i=0,1,2,3,4,5$.

Lemma 4.22. Let $i, i^{\prime}=0,1,2,3,4,5, i \neq i^{\prime}$. The following relations hold:

$$
\begin{gathered}
P_{i} P_{i^{\prime}}=0 \\
P_{i} V P_{i^{\prime}}=0, \\
\left(I-P^{(2)}\right) V P_{i}=\left(I-P^{(2)}\right) V P_{i}^{\partial} .
\end{gathered}
$$

Corollary 4.23. Operators $P^{(2)} V P^{(2)}$ and $P^{(2)} H P^{(2)}$ have a block structure. Namely,

$$
\begin{array}{cc}
P^{(2)} V P^{(2)}=\sum_{i=0}^{5} P_{i} V P_{i}, & P^{(2)} H P^{(2)}=\sum_{i=0}^{5} P_{i} H P_{i} . \\
P_{i} V P_{i}=\sum_{j} P_{i j} V P_{i j}, & P_{i} H P_{i}=\sum_{j} P_{i j} H P_{i j},
\end{array}
$$

\footnotetext{
${ }^{8}$ Unlike polyharmonic situation in [1] here we cannot introduce the corresponding $k^{\delta}$-gap between nonresonant regions or between non-resonant region and all other regions because we can't isolate nontrivial weakly resonant sets. This technical difficulty though can be overcome as in Theorem 4.1, see also the proof of Theorem 5.1 below.
} 
Proof. The lemma easily follows from the construction of the projectors and Lemma 3.26. The identity (258) is simply the definition of the boundary.

Remark. Thus, we have constructed a multiscale structure inside $P^{(2)} H P^{(2)}$, blocks of different colors having distinctly different size. Merging blocks of a smaller size (a lighter color) with neighboring blocks of a bigger size (a darker color), we made the blocks to be separated by the \|\|$\cdot \|||$-distance greater than the size of the corresponding lighter block. This property will be important for the proof of main theorem of the Step III (see Theorem 5.1 below). Absence of the similar property for nonresonant region is partially compensated by the fact that potential $V$ still does not connect different nontrivial weakly resonant sets, while strongly resonant sets are still $k^{\delta}$-separated. Corresponding details were provided in the proof of Theorem 4.1 and will be used again in the proof of Theorem 5.1 .

Lemma 4.24. Let $\varphi_{0} \in \omega^{(2)}(k, \delta, \tau),\left|\varphi-\varphi_{0}\right|<k^{-2-40 r_{1}^{\prime}-\delta}$. Then,

$$
\left\|\left(P_{n r}\left(H\left(\vec{\varkappa}^{(2)}(\varphi)\right)-k^{2} I\right) P_{n r}\right)^{-1}\right\|<c k^{40 r_{1}^{\prime}} .
$$

Proof. The set $\Pi_{n r}$ can be presented as $\cup_{j} \Pi_{n r}^{j}$, each $\Pi_{n r}^{j}$ being a $k^{\delta}$-component. Let $P_{n r}=\sum_{j} P_{n r}^{j}$, where $P_{n r}^{j}$ are projections corresponding to $\Pi_{n r}^{j}$. Then by Corollary 4.23, $P_{n r} H P_{n r}=\sum_{j} P_{n r}^{j} H P_{n r}^{j}$. Hence, it is enough to prove

$$
\left\|\left(P_{n r}^{j}\left(H\left(\vec{\varkappa}^{(2)}(\varphi)\right)-k^{2} I\right) P_{n r}^{j}\right)^{-1}\right\|<c k^{40 r_{1}^{\prime}} .
$$

By construction, each $\Pi_{n r}^{j}$ contains $\mathbf{m} \in \mathcal{M}\left(\varphi, r_{2}\right) \backslash \Omega_{s}^{(2)}, \mathcal{M}\left(\varphi, r_{2}\right)=\mathcal{M}_{1}\left(\varphi, r_{2}\right) \cup \mathcal{M}_{2}\left(\varphi, r_{2}\right)$. We can apply Lemmas [3.22, 3.28 and Corollary [3.29, since they were proven for any $r_{1}$ (no restrictions from above). We take $\varepsilon_{0}=k^{-10 r_{1}^{\prime}}$ in Lemma 3.22, since the distance from $\varphi_{0}$ to the nearest pole of the operator $\left(P_{n r}^{j}\left(H\left(\vec{\varkappa}^{(1)}\left(\varphi_{0}\right)\right)-k^{2} I\right) P_{n r}^{j}\right)^{-1}$ is greater than $k^{-10 r_{1}^{\prime}}$, see (251), and $p_{\mathbf{m}}>k^{-5 r_{1}^{\prime}}$ because $\mathbf{m} \notin \Omega_{s}^{(2)}$. By analogy with Corollary [3.29, we obtain:

$$
\left\|\left(P_{n r}^{j}\left(H\left(\vec{\varkappa}^{(1)}\left(\varphi_{0}\right)\right)-k^{2} I\right) P_{n r}^{j}\right)^{-1}\right\|<c k^{40 r_{1}^{\prime}} .
$$

Taking into account that $\varkappa^{(2)}\left(\varphi_{0}\right)-\varkappa^{(1)}\left(\varphi_{0}\right)=o\left(k^{-1-40 r_{1}^{\prime}}\right)$ and $\vec{\varkappa}^{(2)}(\varphi)-\vec{\varkappa}^{(2)}\left(\varphi_{0}\right)=$ $o\left(k^{-1-40 r_{1}^{\prime}}\right)$, we arrive at (262).

Lemma 4.25. Let $\varphi_{0} \in \omega^{(2)}(k, \delta, \tau)$, and $\left|\varphi-\varphi_{0}\right|<k^{-44 r_{1}^{\prime}-2-\delta}, i=1,2,3$. Then,

1. The number of poles of the resolvent $\left(P_{i}\left(H\left(\vec{\varkappa}^{(2)}(\varphi)\right)-k^{2} I\right) P_{i}\right)^{-1}$ in the disc $\mid \varphi-$ $\varphi_{0} \mid<k^{-44 r_{1}^{\prime}-2-\delta}$ is no greater than $N_{i}^{(1)}$, where $N_{1}^{(1)}=k^{\gamma r_{1}+3}, N_{2}^{(1)}=k^{\gamma r_{1} / 2+\delta_{0} r_{1}}$, $N_{3}^{(1)}=k^{\gamma r_{1} / 6-\delta_{0} r_{1}}$. 
2. Let $\varepsilon$ be the distance to the nearest pole of the resolvent in $\mathcal{W}^{(2)}$ and let $\varepsilon_{0}:=$ $\min \left\{\varepsilon, k^{-11 r_{1}^{\prime}}\right\}$. Then, the following estimates hold:

$$
\begin{gathered}
\left\|\left(P_{i}\left(H\left(\vec{\varkappa}^{(2)}(\varphi)\right)-k^{2} I\right) P_{i}\right)^{-1}\right\|<c k^{44 r_{1}^{\prime}}\left(\frac{k^{-11 r_{1}^{\prime}}}{\varepsilon_{0}}\right)^{N_{i}^{(1)}}, \\
\left\|\left(P_{i}\left(H\left(\vec{\varkappa}^{(2)}(\varphi)\right)-k^{2} I\right) P_{i}\right)^{-1}\right\|_{1}<c k^{44 r_{1}^{\prime}+8 \gamma r_{1}}\left(\frac{k^{-11 r_{1}^{\prime}}}{\varepsilon_{0}}\right)^{N_{i}^{(1)}} .
\end{gathered}
$$

Proof. Let $\Pi$ be a component $\Pi_{b}^{j}, \Pi_{g}^{j}$ or $\Pi_{w}^{j}$ and $P_{\Pi}$ be the corresponding projection. By Lemmas 4.19, 4.20, 4.21 the number $N$ of elements $\mathcal{M}^{(2)} \cap \Pi$ does not exceed $c k^{\gamma r_{1}+3}$. Let us recall that the set $\mathcal{M}^{(2)}$ is defined by the formula (251), where $\mathcal{O}_{\mathbf{m}}^{(2)}$ is the union of open disks of the radius $k^{-10 r_{1}^{\prime}}$ with the centers at poles of the resolvent of $k^{\delta}$-components containing $\vec{\varkappa}^{(1)}\left(\varphi_{0}\right)+\vec{p}_{\mathbf{m}}$. Let us consider $\mathcal{O}_{\Pi}^{(2)}=\cup_{\mathbf{m} \in \Pi \cap \mathcal{M}^{(2)}} \mathcal{O}_{\mathbf{m}}^{(2)}$ and an analogous set consisting of smaller discs: $\tilde{\mathcal{O}}_{\Pi}^{(2)}=\cup_{\mathbf{m} \in \Pi \cap \mathcal{M}^{(2)}} \tilde{\mathcal{O}}_{\mathbf{m}}^{(2)}$, where $\tilde{\mathcal{O}}_{\mathbf{m}}^{(2)}$ have the radius $k^{-11 r_{1}^{\prime}}$. Since $N<c k^{\gamma r_{1}+3}$, the total size of $\tilde{\mathcal{O}}_{\Pi}^{(2)}$ is less than $k^{-11 r_{1}^{\prime}+\gamma r_{1}+3}=o\left(k^{-10 r_{1}^{\prime}}\right)$.

First, assume $\varphi_{0} \notin \tilde{\mathcal{O}}_{\Pi}^{(2)}$. Then, we can prove an estimate analogous to (261). Indeed, let us consider a $k^{\delta}$-component in $\Pi$. We denote the corresponding projection by $P(\mathbf{m})$. By the definitions of $\mathcal{O}_{\mathbf{m}}^{(2)}, \tilde{\mathcal{O}}_{\mathbf{m}}^{(2)}$, the distance from $\varphi_{0}$ to the nearest pole of $\left(P(\mathbf{m})\left(H\left(\vec{\varkappa}^{(1)}\right)-k^{2} I\right) P(\mathbf{m})\right)^{-1}$ is greater than $k^{-11 r_{1}^{\prime}}$. Applying Lemmas 3.22, 3.28, to these resolvents, we obtain (recall that now $p_{\mathbf{m}}>k^{-5 r_{1}^{\prime}}$ ):

$$
\begin{gathered}
\left\|\left(P(\mathbf{m})\left(H\left(\vec{\varkappa}^{(1)}\left(\varphi_{0}\right)\right)-k^{2} I\right) P(\mathbf{m})\right)^{-1}\right\|<c k^{44 r_{1}^{\prime}}, \\
\left\|\left(P(\mathbf{m})\left(H\left(\vec{\varkappa}^{(1)}\left(\varphi_{0}\right)\right)-k^{2} I\right) P(\mathbf{m})\right)^{-1}\right\|_{1}<c k^{44 r_{1}^{\prime}+4 \delta_{*}} .
\end{gathered}
$$

By analogy with Corollary 3.29 ,

$$
\begin{gathered}
\left\|\left(P\left(H\left(\vec{\varkappa}^{(1)}\left(\varphi_{0}\right)\right)-k^{2} I\right) P\right)^{-1}\right\|<c k^{44 r_{1}^{\prime},} \\
\left\|\left(P\left(H\left(\vec{\varkappa}^{(1)}\left(\varphi_{0}\right)\right)-k^{2} I\right) P\right)^{-1}\right\|_{1}<c k^{44 r_{1}^{\prime}} L^{4},
\end{gathered}
$$

where $P$ is the projection onto all $k^{\delta}$-components in $\Pi, L$ is the size of $\Pi$. Next, arguing as in the proof of Theorem 4.1, we show that the perturbation series for the resolvent $\left(P_{\Pi}\left(H\left(\vec{\varkappa}^{(1)}\left(\varphi_{0}\right)\right)-k^{2} I\right) P_{\Pi}\right)^{-1}$ converges when we take $P H\left(\vec{\varkappa}^{(1)}\left(\varphi_{0}\right)\right) P+\left(P_{\Pi}-P\right) H_{0}$ as the unperturbed operator. Therefore,

$$
\left\|\left(P_{\Pi}\left(H\left(\vec{\varkappa}^{(1)}\left(\varphi_{0}\right)\right)-k^{2} I\right) P_{\Pi}\right)^{-1}\right\|<c k^{44 r_{1}^{\prime}}
$$


no poles being inside of the disc. Taking into account that $\left|\varphi-\varphi_{0}\right|<k^{-44 r_{1}^{\prime}-2-\delta}$ and $\left|\vec{\varkappa}^{(2)}-\vec{\varkappa}^{(1)}\right|=o\left(k^{-44 r_{1}^{\prime}-2}\right)$, we obtain

$$
\left\|\left(P_{\Pi}\left(H\left(\vec{\varkappa}^{(2)}(\varphi)\right)-k^{2} I\right) P_{\Pi}\right)^{-1}\right\|<c k^{44 r_{1}^{\prime}} .
$$

By Lemmas 4.19, 4.20, 4.21, $L<k^{2 \gamma r_{1}}$,

$$
\left\|\left(P_{\Pi}\left(H\left(\vec{\varkappa}^{(2)}(\varphi)\right)-k^{2} I\right) P_{\Pi}\right)^{-1}\right\|_{1}<c k^{8 \gamma r_{1}+44 r_{1}^{\prime}} .
$$

Thus, the resolvent has no poles inside the disk around $\varphi_{0}$ and the estimates (264), (265) hold with $\varepsilon_{0}:=k^{-11 r_{1}^{\prime}}$. Second, if $\varphi \notin \tilde{\mathcal{O}}_{\Pi}^{(2)}$, then $\varphi_{0} \notin \tilde{\mathcal{O}}_{\Pi}^{(2)}\left(11 r_{1}^{\prime}, \frac{1}{2}\right)$, the latter set consisting of discs of the radius $\frac{1}{2} k^{-11 r_{1}^{\prime}}$. Therefore the estimates analogous to the last two hold. Now estimates (264), (265) easily follow.

It remains to consider the case $\varphi_{0}, \varphi \in \tilde{\mathcal{O}}_{\Pi}^{(2)}$. Obviously, $\varphi_{0}, \varphi$ belong to the same connected component of $\tilde{\mathcal{O}}_{\Pi}^{(2)}$ or to different components being at the distance less than $k^{-44 r_{1}^{\prime}-2-\delta}$ from each other. We consider a $\varphi_{*} \in \partial \tilde{\mathcal{O}}_{\Pi}^{(2)}$, where $\partial \tilde{\mathcal{O}}_{\Pi}^{(2)}$ is the boundary of the component(s) containing $\varphi_{0}, \varphi$. Note that $\varphi_{*} \notin \mathcal{O}_{\mathbf{m}}^{(2)}\left(11 r_{1}^{\prime}, 1\right)$ for all $\mathbf{m} \in \Pi$. Indeed, for $\mathbf{m} \in \mathcal{M}^{(2)}$, it just follows from the relation $\varphi_{*} \in \partial \tilde{\mathcal{O}}_{\Pi}^{(2)}$ and the definition of an open disk $\mathcal{O}_{\mathbf{m}}^{(2)}\left(11 r_{1}^{\prime}, 1\right)$. If $\mathbf{m} \in \Pi \backslash \mathcal{M}^{(2)}$, then $\varphi_{0}$ is not in $\mathcal{O}_{\mathbf{m}}^{(2)}\left(10 r_{1}^{\prime}, 1\right)$ by the definition of $\mathcal{M}^{(2)}$. Since $\varphi_{0}, \varphi \in \tilde{\mathcal{O}}_{\Pi}^{(2)}$ and the length of $\tilde{\mathcal{O}}_{\Pi}^{(2)}$ is $o\left(k^{-10 r_{1}^{\prime}}\right)$, we have $\varphi_{*} \notin \mathcal{O}_{\mathbf{m}}^{(2)}\left(10 r_{1}^{\prime}, \frac{1}{2}\right)$.

Now, considering as in the case $\varphi_{0} \notin \tilde{\mathcal{O}}_{\Pi}^{(2)}$, we obtain that the perturbation series for the resolvent $\left(P_{\Pi}\left(H\left(\overrightarrow{\mathcal{\varkappa}}^{(1)}\left(\varphi_{*}\right)\right)-k^{2} I\right) P_{\Pi}\right)^{-1}$ converges when we take $P H\left(\overrightarrow{\mathcal{\varkappa}}^{(1)}\left(\varphi_{*}\right)\right) P+$ $\left(P_{\Pi}-P\right) H_{0}$ as the unperturbed operator. Therefore,

$$
\left\|\left(P_{\Pi}\left(H\left(\vec{\varkappa}^{(2)}\left(\varphi_{*}\right)\right)-k^{2} I\right) P_{\Pi}\right)^{-1}\right\|<c k^{44 r_{1}^{\prime}} .
$$

The number of poles of the resolvent $\left(P_{\Pi}\left(H\left(\vec{\varkappa}^{(2)}(\varphi)\right)-k^{2} I\right) P_{\Pi}\right)^{-1}$ in $\tilde{\mathcal{O}}_{\Pi}^{(2)}$ is the same as the number of poles of the resolvent of unperturbed operator. Hence, it is $N$. Using the Maximum principle, we get (264) for the case $\varepsilon \leq k^{-11 r_{1}^{\prime}}$, where $N_{i}=N$ and depends on the color of $\Pi$. Considering that the dimension of $P_{\Pi}$ does not exceed $k^{8 \gamma r_{1}}$, we obtain (265)

At last, let $\Pi_{s}^{j}$ be a particular $k^{r_{1} / 2}$-box around $\mathbf{m} \in \Omega_{s}^{(2)}$. Let $P_{s}^{j}$ be corresponding projection.

Lemma 4.26. Let $\varphi_{0} \in \omega^{(2)}(k, \delta, \tau)$. Then, the operator $\left(P_{s}^{j}\left(H\left(\vec{\varkappa}^{(2)}(\varphi)\right)-k^{2} I\right) P_{s}^{j}\right)^{-1}$ has no more than one pole in the disk $\left|\varphi-\varphi_{0}\right|<k^{-r_{1}^{\prime}-\delta}$. Moreover,

$$
\left\|\left(P_{s}^{j}\left(H\left(\vec{\varkappa}^{(2)}(\varphi)\right)-k^{2} I\right) P_{s}^{j}\right)^{-1}\right\|<\frac{8 k^{-1}}{p_{\mathbf{m}} \varepsilon_{0}},
$$




$$
\left\|\left(P_{s}^{j}\left(H\left(\vec{\varkappa}^{(2)}(\varphi)\right)-k^{2} I\right) P_{s}^{j}\right)^{-1}\right\|_{1}<\frac{8 k^{-1+4 r_{1}}}{p_{\mathbf{m}} \varepsilon_{0}},
$$

$\varepsilon_{0}=\min \left\{\varepsilon, k^{-r_{1}^{\prime}-\delta}\right\}$, where $\varepsilon$ is the distance to the pole of the operator.

Proof. The proof is similar to that of Lemma 3.22 (part 3). Indeed, when $p_{\mathbf{m}}<$ $k^{-5 r_{1}^{\prime}}$, the series for $\lambda^{(2)}\left(\vec{\varkappa}^{(2)}(\varphi)+\vec{p}_{\mathbf{m}}\right)$ converges in the complex $k^{-r_{1}^{\prime}-\delta}$ neighborhood of $\omega^{(2)}(k, \delta, \tau)$ and $\lambda^{(2)}\left(\vec{\varkappa}^{(2)}(\varphi)+\vec{p}_{\mathbf{m}}\right)=\lambda^{(1)}\left(\vec{\varkappa}^{(1)}(\varphi)+\vec{p}_{\mathbf{m}}\right)+o\left(k^{-100 r_{1}}\right)$, see (196)). By Lemma 10.2 (Appendix 4), the equation $\lambda^{(1)}\left(\vec{\varkappa}^{(1)}(\varphi)+\vec{p}_{\mathbf{m}}\right)=k^{2}+\varepsilon_{0},\left|\varepsilon_{0}\right| \leq p_{\mathbf{m}} k^{\delta}$ has no more than two solutions in this neighborhood of $\omega^{(2)}(k, \delta, \tau)$. Using Lemma 10.4 and Rouché's Theorem, we obtain the same fact for $\lambda^{(2)}\left(\vec{\varkappa}^{(2)}(\varphi)+\vec{p}_{\mathbf{m}}\right)=\varepsilon_{0}$. It is easy to show that the analogues of Lemmas 10.3, 10.4 and 10.5 hold for $\lambda^{(2)}\left(\vec{\varkappa}^{(2)}(\varphi)+\vec{p}_{\mathbf{m}}\right)$. Thus, we obtain (268), (269).

\subsubsection{Resonant and Nonresonant Sets for Step III}

We divide $[0,2 \pi)$ into $\left[2 \pi k^{44 r_{1}^{\prime}+2+\delta}\right]+1$ intervals $\Delta_{l}^{(2)}$ with the length not bigger than $k^{-44 r_{1}^{\prime}-2-\delta}$. If a particular interval belongs to $\mathcal{O}^{(2)}$, we ignore it; otherwise, let $\varphi_{0}^{(l)}$ be a point inside the $\Delta_{l}^{(2)}, \varphi_{0}^{(l)} \notin \mathcal{O}^{(2)}$. Let

$$
\mathcal{W}_{l}^{(2)}=\left\{\varphi \in \mathcal{W}^{(2)}:\left|\varphi-\varphi_{0}^{(l)}\right|<4 k^{-44 r_{1}^{\prime}-2-\delta}\right\} .
$$

Clearly, neighboring sets $\mathcal{W}_{l}^{(2)}$ overlap (because of the multiplier 4 in the inequality), they cover $\hat{\mathcal{W}}^{(2)}$, which is the restriction of $\mathcal{W}^{(2)}$ to the $2 k^{-44 r_{1}^{\prime}-2-\delta}$-neighborhood of $[0,2 \pi)$. For each $\varphi \in \hat{\mathcal{W}}^{(2)}$ there is an $l$ such that $\left|\varphi-\varphi_{0}^{(l)}\right|<4 k^{-44 r_{1}^{\prime}-2-\delta}$. For each $\varphi_{0}^{(l)}$ we construct the projection $P^{(2)}$, see (254). Further, we consider the poles of the resolvent $\left(P^{(2)}\left(H\left(\vec{\varkappa}^{(2)}(\varphi)\right)-k^{2}\right) P^{(2)}\right)^{-1}$ in $\hat{\mathcal{W}}_{l}^{(2)}$ and denote them by $\varphi_{l m}^{(2)}, m=1, \ldots, M_{l}, P^{(2)}$ being constructed for $\varphi_{0}=\varphi_{0}^{(l)}$. By Corollary 4.23, the resolvent has a block structure. The number of blocks clearly cannot exceed the number of elements in $\Omega\left(r_{2}\right)$, i.e. $k^{4 r_{2}}$. Using the estimates for the number of poles for each block, the estimate being provided by Lemma 4.25 Part 1, we can roughly estimate the number of poles of the resolvent by $k^{4 r_{2}+r_{1}}$.

Next, let $r_{2}^{\prime}>11 r_{1}^{\prime}$ and $\mathcal{O}_{l m}^{(3)}$ be the disc of the radius $k^{-r_{2}^{\prime}}$ around $\varphi_{m l}^{(2)}$.

Definition 4.27. The set

$$
\mathcal{O}^{(3)}=\cup_{l m} \mathcal{O}_{l m}^{(3)}
$$

we call the third resonant set. The set

$$
\mathcal{W}^{(3)}=\hat{\mathcal{W}}^{(2)} \backslash \mathcal{O}^{(3)}
$$

is called the third non-resonant set. The set

$$
\omega^{(3)}=\mathcal{W}^{(3)} \cap[0,2 \pi)
$$

is called the third real non-resonant set. 
Lemma 4.28. Let $r_{2}^{\prime}>\mu r_{2}>44 r_{1}^{\prime}, \varphi \in \mathcal{W}^{(3)}, \varphi_{0}^{(l)}$ corresponds to an interval $\Delta_{l}^{(2)}$ containing $\Re \varphi$. Let $\Pi$ be one of the components $\Pi_{s}^{j}\left(\varphi_{0}^{(l)}\right), \Pi_{b}^{j}\left(\varphi_{0}^{(l)}\right), \Pi_{g}^{j}\left(\varphi_{0}^{(l)}\right), \Pi_{w}^{j}\left(\varphi_{0}^{(l)}\right)$ and $P(\Pi)$ be the projection corresponding to $\Pi$. Let also $\varkappa \in \mathbb{C}:\left|\varkappa-\varkappa^{(2)}(\varphi)\right|<k^{-r_{2}^{\prime} k^{2 \gamma r_{1}}}$. Then, for $\vec{\varkappa}(\varphi)=\varkappa(\cos \varphi, \sin \varphi)$ the following inequality holds:

$$
\begin{gathered}
\left\|\left(P(\Pi)\left(H(\vec{\varkappa}(\varphi))-k^{2} I\right) P(\Pi)\right)^{-1}\right\|<c k^{2 \mu r_{2}+r_{2}^{\prime} N^{(1)}}, \\
\left\|\left(P(\Pi)\left(H(\vec{\varkappa}(\varphi))-k^{2} I\right) P(\Pi)\right)^{-1}\right\|_{1}<c k^{(2 \mu+1) r_{2}+r_{2}^{\prime} N^{(1)}},
\end{gathered}
$$

$N^{(1)}$ corresponding to the color of $\Pi$ : $N^{(1)}=1, k^{\gamma r_{1}+3}, k^{\gamma r_{1} / 2+\delta_{0} r_{1}}, k^{\gamma r_{1} / 6-\delta_{0} r_{1}}$ for simple, black, grey and white clusters, correspondingly.

Proof. For $\vec{\varkappa}=\vec{\varkappa}^{(2)}(\varphi)$ the lemma follows immediately from the definition of $\mathcal{W}^{(3)}$ and Lemmas 4.25 and $4.26\left(p_{\mathbf{m}}>k^{-2 \mu r_{2}}\right)$. It is easy to see that estimates (274) and (275) are stable with respect to perturbation of $\varkappa^{(2)}$ of order $k^{-r_{2}^{\prime} k^{2 \gamma r_{1}}}$.

By total size of the set $\mathcal{O}^{(3)}$ we mean the sum of the sizes of its connected components.

Lemma 4.29. Let $r_{2}>45 r_{1}^{\prime}+2, r_{2}^{\prime} \geq(\mu+10) r_{2}$. Then, the size of each connected component of $\mathrm{O}^{(3)}$ is less than $k^{5 r_{2}-r_{2}^{\prime}}$. The total size of $\mathcal{O}^{(3)}$ is less than $k^{-r_{2}^{\prime} / 2}$.

Proof. Indeed, each set $\mathcal{W}_{l}^{(2)}$ contains no more than $k^{4 r_{2}+r_{1}}$ discs $\mathcal{O}_{l m}^{(3)}$. Therefore, the size of $\mathcal{O}^{(3)} \cap \mathcal{W}_{l}^{(2)}$ is less than $k^{-r_{2}^{\prime}+5 r_{2}}$. Considering that $k^{-r_{2}^{\prime}+5 r_{2}}$ is much smaller that the length of $\Delta_{l}^{(2)}$, we obtain that there is no connected components which go across the whole set $\mathcal{W}_{l}^{(2)}$ and the size of each connected component of $\mathcal{O}^{(3)}$ is less than $k^{5 r_{2}-r_{2}^{\prime}}$. Considering that the number of intervals $\Delta_{l}^{(2)}$ is less than $k^{45 r_{1}^{\prime}+2+\delta}$, we obtain the required estimate for the total size of $\mathcal{O}^{(3)}$.

Lemma 4.30. Let $\varphi \in \mathcal{W}^{(2)}$ and $C_{3}$ be the circle $\left|z-k^{2}\right|=k^{-2 r_{2}^{\prime} k^{2 \gamma r_{1}}}$. Then

$$
\left\|\left(P\left(r_{1}\right)\left(H\left(\vec{\varkappa}^{(2)}(\varphi)\right)-z\right) P\left(r_{1}\right)\right)^{-1}\right\| \leq 4^{2} k^{2 r_{2}^{\prime} k^{2 \gamma r_{1}}} .
$$

Proof. The proof is similar to the proof of Lemma 3.21 in [1], when we take into account (181) and (202), (203). In the proof we use the estimates from the previous step along with some perturbation arguments. First, we use the series decomposition (178) -(181)) for $\vec{\varkappa}=\vec{\varkappa}^{(2)}(\varphi)$ and $z \in C_{2}$. Then, considering that the resolvent has a single pole in $C_{2}$ located at $z=k^{2}$, and using the Maximum principle, we obtain (276)).

\section{$5 \quad$ Step III}

Let $k_{*}$ be sufficiently large to satisfy the estimates:

$$
k_{*} \geq k_{1}(V, \delta, \tau), \quad k_{*}^{\delta / 8}>10^{8}+\|V\|+\mu+2,
$$


$k_{1}(V, \delta, \tau)$ being introduced in the formulation of Theorem 4.1. We also assume that $k_{*}$ is such that all constants $c$ in previous estimates (e.g. (274), (275)) satisfy $c<k_{*}^{\delta / 8}$. Since now on we consider $k>k_{*}$. This restriction on $k$ won't change in all consecutive steps. We introduce a new notation $O_{T}(\cdot)$ : let $f(k)=O_{T}\left(k^{-\gamma}\right)$ mean that $|f(k)|<T k^{-\gamma}$ when $k>k_{*}$.

\subsection{Operator $H^{(3)}$. Perturbation Formulas}

Let $P\left(r_{2}\right)$ be an orthogonal projector onto $\Omega\left(r_{2}\right):=\left\{\mathbf{m}:\left\|\vec{p}_{\mathbf{m}}\right\| \mid \leq k^{r_{2}}\right\}$ and $H^{(3)}=$ $P\left(r_{2}\right) H P\left(r_{2}\right)$. From now on we assume

$$
k^{\delta}<r_{2}<k^{\gamma 10^{-7} r_{1}} .
$$

Note that $45 r_{1}^{\prime}+2<k^{\delta}<k^{\gamma 10^{-7}} r_{1}$ for all $k>k_{*}$, since $10^{8}<r_{1}<k^{\delta / 8}$, see (165) and above (250). Let $\beta:=\frac{\delta_{*}}{100}$. Let us introduce $r_{2}^{\prime}$, satisfying the inequality:

$$
5 \mu r_{2}<r_{2}^{\prime}<k^{\delta_{0} r_{1}-4}
$$

We consider $H^{(3)}\left(\vec{\varkappa}^{(2)}(\varphi)\right)$ as a perturbation of $\tilde{H}^{(2)}\left(\vec{\varkappa}^{(2)}(\varphi)\right)$ :

$$
\tilde{H}^{(2)}:=\tilde{P}_{l}^{(2)} H \tilde{P}_{l}^{(2)}+\left(P\left(r_{2}\right)-\tilde{P}_{l}^{(2)}\right) H_{0},
$$

where $H=H\left(\vec{\varkappa}^{(2)}(\varphi)\right), H_{0}=H_{0}\left(\vec{\varkappa}^{(2)}(\varphi)\right)$ and $\tilde{P}_{l}^{(2)}$ is the projection $P^{(2)}$, see (255), corresponding to $\varphi_{0}^{(l)}$ in the interval $\Delta_{l}^{(2)}$ containing $\varphi$. Note that the operator $\tilde{H}^{(2)}$ has a block structure, each block $\tilde{P}_{l}^{(2)} H \tilde{P}_{l}^{(2)}$ being composed of smaller blocks $P_{i} H P_{i}, i=0, \ldots, 5$, see (259), (260). Let

$$
\begin{gathered}
W^{(2)}=H^{(3)}-\tilde{H}^{(2)}=P\left(r_{2}\right) V P\left(r_{2}\right)-\tilde{P}_{l}^{(2)} V \tilde{P}_{l}^{(2)}, \\
g_{r}^{(3)}(\vec{\varkappa}):=\frac{(-1)^{r}}{2 \pi i r} \operatorname{Tr} \oint_{C_{3}}\left(W^{(2)}\left(\tilde{H}^{(2)}(\vec{\varkappa})-z I\right)^{-1}\right)^{r} d z, \\
G_{r}^{(3)}(\vec{\varkappa}):=\frac{(-1)^{r+1}}{2 \pi i} \oint_{C_{3}}\left(\tilde{H}^{(2)}(\vec{\varkappa})-z I\right)^{-1}\left(W^{(2)}\left(\tilde{H}^{(2)}(\vec{\varkappa})-z I\right)^{-1}\right)^{r} d z,
\end{gathered}
$$

where $C_{3}$ is the circle $\left|z-k^{2}\right|=\varepsilon_{0}^{(3)}, \varepsilon_{0}^{(3)}=k^{-2 r_{2}^{\prime} k^{2 \gamma r_{1}}}$.

Theorem 5.1. Suppose $k>k_{*}, \varphi$ is in the real $k^{-r_{2}^{\prime}-\delta}$-neighborhood of $\omega^{(3)}(k, \delta, \tau)$ and $\varkappa \in \mathbb{R},\left|\varkappa-\varkappa^{(2)}(\varphi)\right| \leq \varepsilon_{0}^{(3)} k^{-1-\delta}, \vec{\varkappa}=\varkappa(\cos \varphi, \sin \varphi)$. Then, there exists a single eigenvalue of $H^{(3)}(\vec{\varkappa})$ in the interval $\varepsilon_{3}(k, \delta, \tau)=\left(k^{2}-\varepsilon_{0}^{(3)}, k^{2}+\varepsilon_{0}^{(3)}\right)$. It is given by the absolutely converging series:

$$
\lambda^{(3)}(\vec{\varkappa})=\lambda^{(2)}(\vec{\varkappa})+\sum_{r=2}^{\infty} g_{r}^{(3)}(\vec{\varkappa}) .
$$


For coefficients $g_{r}^{(3)}(\overrightarrow{\mathcal{\imath}})$ the following estimates hold:

$$
\left|g_{r}^{(3)}(\vec{\varkappa})\right|<k^{-\frac{\beta}{5} k^{r_{1}-\delta_{*}}-\beta(r-1)} .
$$

The corresponding spectral projection is given by the series:

$$
\mathcal{E}^{(3)}(\vec{\varkappa})=\mathcal{E}^{(2)}(\vec{\varkappa})+\sum_{r=1}^{\infty} G_{r}^{(3)}(\vec{\varkappa})
$$

$\mathcal{E}^{(2)}(\vec{\varkappa})$ being the spectral projection of $H^{(2)}(\vec{\varkappa})$. The operators $G_{r}^{(3)}(\vec{\varkappa})$ satisfy the estimates:

$$
\begin{gathered}
\left\|G_{r}^{(3)}(\vec{\varkappa})\right\|_{1}<k^{-\frac{\beta}{10} k^{r_{1}-\delta *}-\beta r}, \\
G_{r}^{(3)}(\vec{\varkappa})_{\mathbf{s s}^{\prime}}=0, \text { when } 2 r k^{\gamma r_{1}+3}+3 k^{r_{1}}<\left\|\left|\vec{p}_{\mathbf{s}}\|\|+\left\|\mid \vec{p}_{\mathbf{s}^{\prime}}\right\| \| .\right.\right.
\end{gathered}
$$

Corollary 5.2. For the perturbed eigenvalue and its spectral projection the following estimates hold:

$$
\begin{gathered}
\lambda^{(3)}(\vec{\varkappa})=\lambda^{(2)}(\vec{\varkappa})+O_{2}\left(k^{-\frac{\beta}{5} k^{r_{1}-\delta_{*}}}\right), \\
\left\|\mathcal{E}^{(3)}(\vec{\varkappa})-\mathcal{E}^{(2)}(\vec{\varkappa})\right\|_{1}<k^{-\frac{\beta}{10} k^{r_{1}-\delta_{*}}}, \\
\left|\mathcal{E}^{(3)}(\vec{\varkappa})_{\mathbf{s s}^{\prime}}\right|<k^{-d^{(3)}\left(\mathbf{s}, \mathbf{s}^{\prime}\right)}, \quad \text { when }\left\|\left|\vec{p}_{\mathbf{s}} \|\right|>4 k^{r_{1}} \text { or }\right\|\left|\vec{p}_{\mathbf{s}^{\prime}} \|\right|>4 k^{r_{1}}, \\
d^{(3)}\left(\mathbf{s}, \mathbf{s}^{\prime}\right)=\frac{1}{16}\left(\left\|\left|\vec { p } _ { \mathbf { s } } \left\|\left|+\left\|\left|\vec{p}_{\mathbf{s}^{\prime}} \|\right|\right) k^{-\gamma r_{1}-3} \beta+\frac{\beta}{10} k^{r_{1}-\delta_{*}} .\right.\right.\right.\right.\right.
\end{gathered}
$$

Formulas (287) and (288) easily follow from (282), (283) and (284), (285). The estimate (289) follows from (284), (285) and (286).

Proof. The proof will follow the constructions from the proof of Theorem 4.1. Let us consider the perturbation series

$$
\left(H^{(3)}-z\right)^{-1}=\sum_{r=0}^{\infty}\left(\tilde{H}^{(2)}-z\right)^{-1}\left(-W^{(2)}\left(\tilde{H}^{(2)}-z\right)^{-1}\right)^{r},
$$

here and below all the operators are computed at $\vec{\varkappa}$. Further, we consider $\vec{\varkappa}$ and, therefore, the operators, as analytic functions of $\varphi$ in $\mathcal{W}_{l}^{(2)}$, assuming $\varkappa$ is fixed. By (279) and (259), $W^{(2)}=P\left(r_{2}\right)\left(V-\sum_{i=0}^{5} P_{i} V P_{i}\right) P\left(r_{2}\right)$. By assumption on $\varkappa$ and Lemmas 3.23, 4.28 and 4.30 ,

$$
\left\|\left(\tilde{H}^{(2)}(\vec{\varkappa})-z\right)^{-1}\right\|<2 \cdot 4^{2} k^{2 r_{2}^{\prime} k^{2 \gamma r_{1}}} .
$$

To check the convergence it is enough to show that

$$
\left\|\left(\tilde{H}^{(2)}-z\right)^{-1} W^{(2)}\left(\tilde{H}^{(2)}-z\right)^{-1}\right\|<k^{-2 \beta} .
$$


Then,

$$
\left\|\left(H^{(3)}(\vec{\varkappa})-z\right)^{-1}\right\|<4^{3} k^{2 r_{2}^{\prime} k^{2 \gamma r_{1}}} .
$$

Let us prove (292). Operator $\tilde{H}^{(2)}$ has a block structure. Identities (257) imply that not only the blocks themselves, but also the blocks multiplied by $W^{(2)}$ have zero action on orthogonal subspaces. The operator $\tilde{H}^{(2)}$ acts as $H_{0}$ "outside" the blocks. Because of the block structure, Corollary 4.23 and (258), it suffices to prove:

$$
\begin{gathered}
\left\|\left(H_{0}-z\right)^{-1}\left(P\left(r_{2}\right)-\tilde{P}_{l}^{(2)}\right) V\left(P\left(r_{2}\right)-\tilde{P}_{l}^{(2)}\right)\left(H_{0}-z\right)^{-1}\right\| \leq k^{-\delta_{*} / 8}<k^{-3 \beta}, \\
\left\|\left(H_{0}-z\right)^{-1}\left(P\left(r_{2}\right)-\tilde{P}_{l}^{(2)}\right) V P^{\partial}\left(r_{1}\right)\left(\tilde{H}^{(2)}-z\right)^{-1}\right\| \leq k^{-\delta_{*} / 8+215 \mu \delta}<k^{-3 \beta}, \\
\left\|\left(H_{0}-z\right)^{-1}\left(P\left(r_{2}\right)-\tilde{P}_{l}^{(2)}\right) V P_{n r}^{\partial}\left(\tilde{H}^{(2)}-z\right)^{-1}\right\| \leq k^{-\delta_{*} / 8}<k^{-3 \beta}, \\
\left\|\left(H_{0}-z\right)^{-1}\left(P\left(r_{2}\right)-\tilde{P}_{l}^{(2)}\right) V P_{s}^{\partial}\left(\tilde{H}^{(2)}-z\right)^{-1}\right\| \leq k^{-\delta_{*} / 8+215 \mu \delta}<k^{-3 \beta}, \\
\left\|\left(H_{0}-z\right)^{-1}\left(P\left(r_{2}\right)-\tilde{P}_{l}^{(2)}\right) V P_{w}^{\partial}\left(\tilde{H}^{(2)}-z\right)^{-1}\right\| \leq k^{-\delta_{*} / 8+215 \mu \delta}<k^{-3 \beta}, \\
\left\|\left(H_{0}-z\right)^{-1}\left(P\left(r_{2}\right)-\tilde{P}_{l}^{(2)}\right) V P_{g}^{\partial}\left(\tilde{H}^{(2)}-z\right)^{-1}\right\| \leq k^{-\delta_{*} / 8+430 \mu \delta}<k^{-3 \beta}, \\
\left\|\left(H_{0}-z\right)^{-1}\left(P\left(r_{2}\right)-\tilde{P}_{l}^{(2)}\right) V P_{b}^{\partial}\left(\tilde{H}^{(2)}-z\right)^{-1}\right\| \leq k^{-\delta_{*} / 8+645 \mu \delta}<k^{-3 \beta} .
\end{gathered}
$$

By definition of $\tilde{P}_{l}^{(2)}$, a matrix element $\left(P\left(r_{2}\right)-\tilde{P}_{l}^{(2)}\right)_{\text {mm }}$ can differ from zero only if $\mathbf{m}$ is not in $\mathcal{M}$ or in a trivial weakly resonant set. Considering as in the proof of (180), we obtain (294).

Let us prove (295)). We notice that $P\left(r_{1}\right) \tilde{H}^{(2)}=H^{(2)}$. As in the proof of Theorem 4.1, we consider $H^{(2)}$ as a perturbation of $\tilde{H}^{(1)}, H^{(2)}=\tilde{H}^{(1)}+W$. Taking into account that $\tilde{H}^{(1)}$ has a block structure and $V$ is a trigonometric polynomial, we obtain

$$
P^{\partial}\left(r_{1}\right)\left(\left(\tilde{H}^{(1)}-z\right)^{-1} W\right)^{s} P(\delta)=0, \quad \text { when } 1 \leq s \leq S, \quad S:=\left[k^{r_{1}-\delta_{*}-\delta}\right] .
$$

Hence,

$$
\begin{aligned}
& P^{\partial}\left(r_{1}\right)\left(H^{(2)}-z\right)^{-1} P\left(r_{1}\right)=\sum_{s=0}^{S-1} P^{\partial}\left(r_{1}\right)\left(-\left(\tilde{H}^{(1)}-z\right)^{-1} W \hat{P}\right)^{s}\left(\tilde{H}^{(1)}-z\right)^{-1} \hat{P} \\
& +P^{\partial}\left(r_{1}\right)\left(-\left(\tilde{H}^{(1)}-z\right)^{-1} W \hat{P}\right)^{S}\left(H^{(2)}-z\right)^{-1}
\end{aligned}
$$


where $\hat{P}=P\left(r_{1}\right)-P(\delta)$. Considering as in the proof of (180) (here, we replace $P\left(r_{1}\right)$ by $\hat{P}$, this compensates for the smallness of $C_{3}$ ), we obtain:

$$
\left\|\hat{P}\left(\tilde{H}^{(1)}-z\right)^{-1} W \hat{P}\left(\tilde{H}^{(1)}-z\right)^{-1}\right\|<c k^{-\delta_{*} / 8} .
$$

Next, by Theorem 4.1 and the definition of $C_{3},\left\|\left(\tilde{H}^{(2)}-z\right)^{-1}\right\|<\left(\varepsilon_{0}^{(3)}\right)^{-1}<k^{\delta_{*} S / 20}$. Substituting the last two estimates into (301), we obtain:

$$
P^{\partial}\left(r_{1}\right)\left(H^{(2)}-z\right)^{-1} P\left(r_{1}\right)=P^{\partial}\left(r_{1}\right)\left(\tilde{H}^{(1)}-z\right)^{-1}\left(I+O\left(k^{-\delta_{*} / 8}\right)\right)+O\left(k^{-\delta_{*} / 8}\right) .
$$

Again, considering as in the proof of (180), we get:

$$
\left\|\left(H_{0}-z\right)^{-1}\left(P\left(r_{2}\right)-\tilde{P}_{l}^{(2)}\right) V P^{\partial}\left(r_{1}\right)\left(\tilde{H}^{(1)}-z\right)^{-1}\right\|<c k^{-\delta_{*} / 8} .
$$

The estimate (295) follows from the last two estimates.

The proof of (296) is analogous to the proof of (180), where one uses Lemma 4.24 rather than Corollary 3.29 .

Next, we prove (297). Denote by $\hat{H}$ the reduction of the operator $H$ onto a particular simple cluster i.e. $\hat{H}=P_{s} H P_{s}$ where $\left(P_{s}\right)_{\mathbf{m m}}=1$, if $\mathbf{m}$ belongs to this simple cluster and $\left(P_{s}\right)_{\mathrm{mm}}=0$ otherwise. By Lemma 4.28 (and obvious perturbation arguments to replace $k^{2}$ by $\left.z\right)$,

$$
\left\|(\hat{H}-z)^{-1}\right\| \leq c k^{2 \mu r_{2}+r_{2}^{\prime}} .
$$

We are going to construct a perturbation formula for $P_{s}^{\partial}(\hat{H}-z)^{-1} P_{s}$. Let $\hat{H}_{0}=P_{s, n r} H P_{s, n r}+$ $\left(P_{s}-P_{s, n r}\right) H_{0}$, where $P_{s, n r}=P_{s} P_{n r}=P_{n r} P_{s}$. Operator $\hat{H}_{0}$ has a block structure. It is analogous to operator $\tilde{H}^{(1)}$ in the proof of Theorem 4.1. The perturbation formula for $P_{s}^{\partial}(\hat{H}-z)^{-1} P_{s}$ has the form:

$$
\begin{aligned}
& P_{s}^{\partial}(\hat{H}-z)^{-1} P_{s}=\sum_{r=0}^{R_{s}} P_{s}^{\partial}\left[-\left(\hat{H}_{0}-z\right)^{-1} W_{s}\right]^{r}\left(\hat{H}_{0}-z\right)^{-1} P_{s} \\
& +P_{s}^{\partial}\left[-\left(\hat{H}_{0}-z\right)^{-1} W_{s}\right]^{R_{s}+1}(\hat{H}-z)^{-1} P_{s}, \\
& W_{s}=\hat{H}-\hat{H}_{0}=P_{s} V P_{s}-P_{s, n r} V P_{s, n r}, \quad R_{s}=\left[k^{\frac{r_{1}}{2}-\delta_{*}-\delta}\right] .
\end{aligned}
$$

Recall that when $\vec{p}_{\mathbf{m}^{\prime}}$ belongs to the boundary of a simple cluster, the $\||\cdot \||$-distance from $\vec{p}_{\mathbf{m}^{\prime}}$ to the point $\vec{p}_{\mathbf{m}}: 0<p_{\mathbf{m}}<k^{-5 r_{1}^{\prime}}$ is at least $k^{r_{1} / 2}-Q$. Since $\vec{p}_{\mathbf{m}}$ is small, the series (304) is analogous to (301), $r_{1}$ being replaced by $r_{1} / 2$. We also notice that (see (303)) $k^{2 \mu r_{2}+r_{2}^{\prime}}<<k^{\delta_{*} R_{s} / 20}$ by (277), (278). Now (297) easily follows.

Now, we prove (298). Here and in what follows we often use the same notation for objects formally different, but playing similar roles in different parts of the proof. We hope 
it will not lead to confusion, but rather make it easier to keep the whole construction and further inductive arguments in mind. Denote by $\hat{H}$ the reduction of the operator $H$ onto a particular white cluster i.e. $\hat{H}=P H P$ where $P_{\mathbf{m m}}=1$ if $\mathbf{m}$ belongs to the white cluster and $P_{\mathbf{m m}}=0$ otherwise. By Lemma 4.28 and obvious perturbative arguments to replace $k^{2}$ by $z$,

$$
\left\|(\hat{H}-z)^{-1}\right\| \leq c k^{2 \mu r_{2}+r_{2}^{\prime} k^{\frac{\gamma r_{1}}{6}-\delta_{0} r_{1}}} .
$$

We are going to construct a perturbation formula for $P_{w}(\hat{H}-z)^{-1} P_{w}^{\partial}$. Let $\hat{H}_{0}=$ $P_{w, n r} H P_{w, n r}+\left(P_{w}-P_{w, n r}\right) H_{0}$, where $P_{w, n r}=P_{w} P_{n r}=P_{n r} P_{w}$. Again, operator $\hat{H}_{0}$ has a block structure analogous to the one of the operator $\tilde{H}^{(1)}$. The perturbation formula for $P_{w}(\hat{H}-z)^{-1} P_{w}^{\partial}$ has the form :

$$
\begin{aligned}
& P_{w}^{\partial}(\hat{H}-z)^{-1} P_{w}=\sum_{r=0}^{R_{w}} P_{w}^{\partial}\left[-\left(\hat{H}_{0}-z\right)^{-1} W\right]^{r}\left(\hat{H}_{0}-z\right)^{-1} P_{w} \\
& +P_{w}^{\partial}\left[-\left(\hat{H}_{0}-z\right)^{-1} W\right]^{R_{w}+1}(\hat{H}-z)^{-1} P_{w} \\
& W=\hat{H}-\hat{H}_{0}, \quad R_{w}=\left[k^{\frac{\gamma r_{1}}{6}-\delta_{*}-\delta}\right] .
\end{aligned}
$$

When $\vec{p}_{\mathbf{m}^{\prime}}$ belongs to the boundary of the white cluster, the $\left\||\cdot \||\right.$-distance from $\vec{p}_{\mathbf{m}^{\prime}}$ to the closest point in $\mathcal{M}^{(2)}$ is $k^{\gamma r_{1} / 6}$. Notice that $\left(\hat{H}_{0}-z\right)_{\mathbf{m m}^{\prime}}^{-1}=0$ if $\left\|\left|\vec{p}_{\mathbf{m}}-\vec{p}_{\mathbf{m}^{\prime}} \|\right|>k^{\delta_{*}+\delta}\right.$. Considering that $R_{w}<k^{\frac{\gamma r_{1}}{6}-\delta_{*}-\delta}$ (so, we never reach the points in $\mathcal{M}^{(2)}$ ), we obtain that the finite sum in (306) can be estimated as those in (301) and (304). Arguing as in the proof of (295) and taking into account that $k^{2 \mu r_{2}+r_{2}^{\prime} k^{\frac{\gamma r_{1}}{6}-\delta_{0} r_{1}}}<<k^{\delta_{*} R_{w} / 20}$, we arrive at (298).

Now, we prove (299). Denote a component of the grey region by $\Pi$ and its boundary (see convention above) by $\partial \Pi$. Corresponding projectors are denoted by $P$ and $P^{\partial}$ respectively. Denote by $\hat{H}$ the reduction of the operator $H$ onto a particular grey cluster i.e. $\hat{H}=P H P$. We are going to construct the perturbation formula for $P^{\partial}(\hat{H}-z)^{-1} P$. Recall, that the size of the neighborhood of grey boxes is $D=k^{\frac{\gamma r_{1}}{2}+2 \delta_{0} r_{1}}$. Let $P_{i}$ be a projector corresponding to a white or non-resonant cluster laying inside $\frac{D}{2}$-neighborhood of $\partial \Pi$, the size of these clusters being much smaller than the size of the neighborhood. For definiteness, let $i=1, \ldots, \hat{I}$. Let $P^{(i n t)}$ be the projector onto all points in $\Pi$ which are at least $D / 2$ away of the boundary (internal points). Note that $P_{i} P^{(i n t)}=0$. At last, put

$$
P_{0}:=P-P^{(i n t)}-\sum_{i=1}^{\hat{I}} P_{i} .
$$

Denote (cf. the case of a white cluster)

$$
\hat{H}_{0}:=\sum_{i=1}^{\hat{I}} P_{i} H P_{i}+P^{(i n t)} H P^{(i n t)}+H_{0} P_{0},
$$




$$
W=\hat{H}-\hat{H}_{0}=P V P-\sum_{i=1}^{\hat{I}} P_{i} V P_{i}-P^{(i n t)} V P^{(i n t)} .
$$

We consider $\hat{H}$ as a perturbation of $\hat{H}_{0}$. Let $R$ be the smallest natural number for which

$$
\mathcal{A}_{R}:=P^{\partial}\left[\left(\hat{H}_{0}-z\right)^{-1} W\right]^{R+1} P^{(i n t)} \neq 0, \quad W:=\hat{H}-\hat{H}_{0} .
$$

It is proven in Appendix 6 that $R>k^{\left(\frac{\gamma}{2}+2 \delta_{0}\right) r_{1}-\delta_{*}-2 \delta}$. Therefore,

$$
\begin{aligned}
P^{\partial}(\hat{H}-z)^{-1} P= & \sum_{r=0}^{R-1} P^{\partial}\left[-\left(\hat{H}_{0}-z\right)^{-1} W\left(P-P^{(i n t)}\right)\right]^{r}\left(\hat{H}_{0}-z\right)^{-1} \\
& +P^{\partial}\left[-\left(\hat{H}_{0}-z\right)^{-1} W\left(P-P^{(i n t)}\right)\right]^{R}(\hat{H}-z)^{-1} P .
\end{aligned}
$$

Again, we plan to proceed as in the proof of (295). However, here the operator $\hat{H}_{0}$ contains not only nonresonant clusters, but also white clusters. Hence, to obtain the estimate

$$
\left\|\left(P-P^{(i n t)}\right)\left(\hat{H}_{0}-z\right)^{-1} W\left(P-P^{(i n t)}\right)\left(\hat{H}_{0}-z\right)^{-1}\right\| \leq k^{-\delta_{*} / 8+215 \mu \delta}
$$

we use (302) (or just (296) ) and already proven estimate (298) for white clusters. Now, we notice that by Lemma 4.28 and perturbation arguments,

$$
\left\|(\hat{H}-z)^{-1}\right\| \leq c k^{2 \mu r_{2}+r_{2}^{\prime} k^{\left(\frac{\gamma}{2}+\delta_{0}\right) r_{1}}} .
$$

Considering that $2 r_{2}^{\prime}<k^{\delta_{0} r_{1}-2 \delta_{*}}$ and combining the estimates above, we obtain (299) in the same way as in the proof of (295).

We prove (300) in the analogous way. Indeed, denote a component of the black region by $\Pi$ and its boundary (see convention above) by $\partial \Pi$. Corresponding projectors are denoted by $P$ and $P^{\partial}$ respectively, $\hat{H}:=P H P$. We and construct the perturbation formula for $P^{\partial}(\hat{H}-z)^{-1} P$. Recall, that the size of the neighborhood of black boxes is $D=k^{\gamma r_{1}+\delta_{0} r_{1}}$. Let $P_{i}$ be a projector corresponding to a grey, white or non-resonant cluster laying inside $\frac{D}{2}$-neighborhood of $\partial \Pi$, the size of these clusters being much smaller than the size of the neighborhood. For definiteness, let $i=1, \ldots, \hat{I}$. Let $P^{(i n t)}$ be the projector onto all points in $\Pi$ which are at least $D / 2$ away of the boundary (internal points). Again, we define $P_{0}, \hat{H}_{0}$ and $W$ by formulas (307), (308) and (309). We are going to use perturbation arguments between $\hat{H}_{0}$ and $\hat{H}$. Let $R$ be the smallest positive integer for which (310) holds in the case of a black cluster. It is proven in Appendix 7 that $R>k^{\left(\gamma r_{1}+\delta_{0} r_{1}-\delta_{*}-2 \delta\right)}$. Next, we use (311). The first term in the RHS of (311) contains only non-resonant, white and grey clusters. Thus, we can use the estimates (296), (298) and (299) obtained before in the case of non-resonant, white and grey clusters. We get

$$
\left\|\left(P-P^{(i n t)}\right)\left(\hat{H}_{0}-z\right)^{-1} W\left(P-P^{(i n t)}\right)\left(\hat{H}_{0}-z\right)^{-1}\right\| \leq k^{-\delta_{*} / 8+430 \mu \delta} .
$$


By Lemma 4.28 and perturbation arguments,

$$
\left\|(\hat{H}-z)^{-1}\right\| \leq c k^{2 \mu r_{2}+r_{2}^{\prime} k^{\gamma r_{1}+3}} .
$$

Considering that $2 r_{2}^{\prime}<k^{\delta_{0} r_{1}-3-2 \delta_{*}}$ and combining the estimates above we obtain (300) in the same way as above.

Estimates (294) - (300) provide convergence of the series for the resolvent. Integrating the resolvent over the contour we get (282) and (284).

Proof of (285) is analogous to that of (173) in Theorem 4.1, Indeed, we consider the operator $A=W^{(2)}\left(\tilde{H}^{(2)}-z\right)^{-1}$ and represent it as $A=A_{0}+A_{1}+A_{2}$, where $A_{0}=\left(P\left(r_{2}\right)-\mathcal{E}^{(2)}(\vec{\varkappa})\right) A\left(P\left(r_{2}\right)-\mathcal{E}^{(2)}(\vec{\varkappa})\right), A_{1}=\left(P\left(r_{2}\right)-\mathcal{E}^{(2)}(\vec{\varkappa})\right) A \mathcal{E}^{(2)}(\vec{\varkappa}), A_{2}=$ $\mathcal{E}^{(2)}(\vec{\varkappa}) A\left(P\left(r_{2}\right)-\mathcal{E}^{(2)}(\vec{\varkappa})\right)$. Note that $\mathcal{E}^{(2)}(\vec{\varkappa}) W^{(2)} \mathcal{E}^{(2)}(\vec{\varkappa})=0$, because of (279)). We see that

$$
\oint_{C_{3}}\left(\tilde{H}^{(2)}-z\right)^{-1} A_{0}^{r} d z=0
$$

since the integrand is a holomorphic function inside $C_{3}$. Therefore,

$$
G_{r}^{(3)}(\vec{\varkappa})=\frac{(-1)^{r}}{2 \pi i} \sum_{j_{1}, \ldots j_{r}=0,1,2, j_{1}^{2}+\ldots+j_{r}^{2} \neq 0} \oint_{C_{3}}\left(\tilde{H}^{(2)}-z\right)^{-1} A_{j_{1}} \ldots . . A_{j_{r}} d z .
$$

At least one of indices in each term is equal to 1 or 2 . Let us show that

$$
\left\|A_{2}\right\|_{1}<k^{-\beta k^{r_{1}-\delta_{*}}+215 \mu \delta} .
$$

Using (279) and the obvious relation $\mathcal{E}^{(2)}=\mathcal{E}^{(2)} P\left(r_{1}\right)$, we obtain:

$$
\begin{aligned}
& \mathcal{E}^{(2)} W^{(2)}\left(P\left(r_{2}\right)-\mathcal{E}^{(2)}\right)=\mathcal{E}^{(2)} P\left(r_{1}\right) W^{(2)}\left(P\left(r_{2}\right)-\tilde{P}_{l}^{(2)}\right)= \\
& \mathcal{E}^{(2)} P^{\partial}\left(r_{1}\right) W^{(2)}\left(P\left(r_{2}\right)-\tilde{P}_{l}^{(2)}\right) .
\end{aligned}
$$

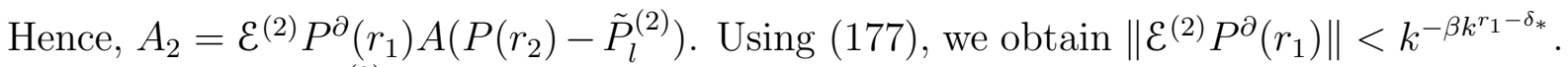
Considering that $\mathcal{E}^{(2)}$ is a one-dimensional projection, we obtain the same estimate for $\mathbf{S}_{\mathbf{1}}$-norm. Using (97) and (117), we obtain

$$
\left\|A\left(P\left(r_{2}\right)-\tilde{P}_{l}^{(2)}\right)\right\| \leq k^{215 \mu \delta} .
$$

Now (316) easily follows. Applying the same considerations as in the proof of (173) with the estimates (291), (292) and (278), we obtain (285).

Let us obtain the estimate for $g_{r}^{(3)}(\vec{\varkappa})$. Obviously,

$$
g_{r}^{(3)}(\vec{\varkappa})=\frac{(-1)^{r}}{2 \pi i r} \sum_{j_{1}, \ldots j_{r}=0,1,2, j_{1}^{2}+\ldots+j_{r}^{2} \neq 0} \operatorname{Tr} \oint_{C_{3}} A_{j_{1} \ldots . . A_{j_{r}}} d z .
$$


Note that each term contains both $A_{1}$ and $A_{2}$, since we compute the trace of the integral. Using (316) and repeating arguments from the proof of (171), we obtain (283).

The estimate (286) follows from the fact that the biggest white, grey or black component has the size not greater than $k^{\gamma r_{1}+3}$. Therefore the biggest block of $\tilde{H}^{(2)}$ not coinciding with $P\left(r_{1}\right) H P\left(r_{1}\right)$ has the size not greater than $k^{\gamma r_{1}+3}$.

It is easy to see that coefficients $g_{r}^{(3)}(\vec{\varkappa})$ and operators $G_{r}^{(3)}(\vec{\varkappa})$ can be analytically extended into the complex $k^{-r_{2}^{\prime}-\delta}$ neighborhood of $\omega^{(3)}$ (in fact, into $k^{-r_{2}^{\prime}-\delta}$-neighborhood of $\left.\mathcal{W}^{(3)}\right)$ as functions of $\varphi$ and to the complex $\left(\varepsilon_{0}^{(3)} k^{-1-\delta}\right)$-neighborhood of $\varkappa=\varkappa^{(2)}(\varphi)$ as functions of $\varkappa$, estimates (283), (285) being preserved. Now, we use formulae (280), (282) to extend $\lambda^{(3)}(\vec{\varkappa})=\lambda^{(3)}(\varkappa, \varphi)$ as an analytic function. Obviously, series (282) is differentiable. Using Cauchy integral and Lemma 4.3 we get the following lemma.

Lemma 5.3. Under conditions of Theorem 5.1 the following estimates hold when $\varphi \in$ $\omega^{(3)}(k, \delta)$ or its complex $k^{-r_{2}^{\prime}-\delta}$-neighborhood and $\varkappa \in \mathbb{C}:\left|\varkappa-\varkappa^{(2)}(\varphi)\right|<\varepsilon_{0}^{(3)} k^{-1-\delta}$.

$$
\begin{gathered}
\lambda^{(3)}(\vec{\varkappa})=\lambda^{(2)}(\vec{\varkappa})+O_{2}\left(k^{-\frac{\beta}{5} k^{r_{1}-\delta_{*}}}\right) \\
\frac{\partial \lambda^{(3)}}{\partial \varkappa}=\frac{\partial \lambda^{(2)}}{\partial \varkappa}+O_{2}\left(k^{-\frac{\beta}{5} k^{r_{1}-\delta_{*}}} M_{1}\right), \quad M_{1}:=\frac{k^{1+\delta}}{\varepsilon_{0}^{(3)}} \\
\frac{\partial \lambda^{(3)}}{\partial \varphi}=\frac{\partial \lambda^{(2)}}{\partial \varphi}+O_{2}\left(k^{-\frac{\beta}{5} k^{r_{1}-\delta_{*}}+r_{2}^{\prime}+\delta}\right) \\
\frac{\partial^{2} \lambda^{(3)}}{\partial \varkappa^{2}}=\frac{\partial^{2} \lambda^{(2)}}{\partial \varkappa^{2}}+O_{2}\left(k^{-\frac{\beta}{5} k^{r_{1}-\delta_{*}}} M_{1}^{2}\right) \\
\frac{\partial^{2} \lambda^{(3)}}{\partial \varkappa \partial \varphi}=\frac{\partial^{2} \lambda^{(2)}}{\partial \varkappa \partial \varphi}+O_{2}\left(k^{-\frac{\beta}{5} k^{r_{1}-\delta_{*}}+r_{2}^{\prime}+\delta} M_{1}\right) \\
\frac{\partial^{2} \lambda^{(3)}}{\partial \varphi^{2}}=\frac{\partial^{2} \lambda^{(2)}}{\partial \varphi^{2}}+O_{2}\left(k^{\left.-\frac{\beta}{5} k^{r_{1}-\delta_{*}+2 r_{2}^{\prime}+2 \delta}\right)}\right.
\end{gathered}
$$

Corollary 5.4. All "O $\mathrm{O}_{2}$ "-s on the right hand sides of (318)-(323) can be written as $O_{1}\left(k^{-\frac{\beta}{10} k^{r_{1}-\delta_{*}}}\right)$.

\subsection{Isoenergetic Surface for Operator $H^{(3)}$}

Lemma 5.5. 1. For every $\lambda:=k^{2}, k>k_{*}$, and $\varphi$ in the real $\frac{1}{2} k^{-r_{2}^{\prime}-\delta}$-neighborhood of $\omega^{(3)}(k, \delta, \tau)$, there is a unique $\varkappa^{(3)}(\lambda, \varphi)$ in the interval

$$
I_{2}:=\left[\varkappa^{(2)}(\lambda, \varphi)-\varepsilon_{0}^{(3)} k^{-1-\delta}, \varkappa^{(2)}(\lambda, \varphi)+\varepsilon_{0}^{(3)} k^{-1-\delta}\right]
$$

such that

$$
\lambda^{(3)}\left(\vec{\varkappa}^{(3)}(\lambda, \varphi)\right)=\lambda, \quad \vec{\varkappa}^{(3)}(\lambda, \varphi):=\varkappa^{(3)}(\lambda, \varphi) \vec{\nu}(\varphi) .
$$


2. Furthermore, there exists an analytic in $\varphi$ continuation of $\varkappa^{(3)}(\lambda, \varphi)$ to the complex $\frac{1}{2} k^{-r_{2}^{\prime}-\delta}$-neighborhood of $\omega^{(3)}(k, \delta, \tau)$ such that $\lambda^{(3)}\left(\vec{\varkappa}^{(3)}(\lambda, \varphi)\right)=\lambda$. Function $\varkappa^{(3)}(\lambda, \varphi)$ can be represented as $\varkappa^{(3)}(\lambda, \varphi)=\varkappa^{(2)}(\lambda, \varphi)+h^{(3)}(\lambda, \varphi)$, where

$$
\begin{gathered}
\left|h^{(3)}(\varphi)\right|=O_{1}\left(k^{-\frac{\beta}{5} k^{r_{1}-\delta_{*}}-1}\right), \\
\frac{\partial h^{(3)}}{\partial \varphi}=O_{2}\left(k^{-\frac{\beta}{5} k^{r_{1}-\delta_{*}}-1+r_{2}^{\prime}+\delta}\right), \quad \frac{\partial^{2} h^{(3)}}{\partial \varphi^{2}}=O_{4}\left(k^{-\frac{\beta}{5} k^{r_{1}-\delta_{*}}-1+2 r_{2}^{\prime}+2 \delta}\right) .
\end{gathered}
$$

Proof. The proof is completely analogous to that of Lemma 3.11, estimates (318) $-(323)$ being used.

Let us consider the set of points in $\mathbb{R}^{2}$ given by the formula: $\vec{\varkappa}=\vec{\varkappa}^{(3)}(\varphi), \quad \varphi \in$ $\omega^{(3)}(k, \delta, \tau)$. By Lemma 5.5 this set of points is a slight distortion of $\mathcal{D}_{2}$. All the points of this curve satisfy the equation $\lambda^{(3)}\left(\vec{\varkappa}^{(3)}(\varphi)\right)=k^{2}$. We call it isoenergetic surface of the operator $H^{(3)}$ and denote by $\mathcal{D}_{3}$.

\subsection{Preparation for Step IV}

\subsubsection{Properties of the Quasiperiodic Lattice. Continuation}

Let

$$
\mathcal{S}^{(2)}(k, \xi):=\left\{\vec{\varkappa} \in \mathbb{R}^{2}:\left\|\left(H^{(2)}(\vec{\varkappa})-k^{2}\right)^{-1}\right\|>k^{\xi}\right\} .
$$

The main purpose of this section is to estimate the number of points $\vec{\varkappa}_{0}+\vec{p}_{\mathbf{m}},\left\|\left|\vec{p}_{\mathbf{m}} \|\right|<k^{r_{2}}\right.$ in $\mathcal{S}^{(2)}(k, \xi), \vec{\varkappa}_{0}$ being fixed. In fact, we prove a more subtle result, see Lemma 5.14.

We consider $\vec{p}_{\mathbf{m}}=2 \pi\left(\mathbf{s}_{1}+\alpha \mathbf{s}_{2}\right)$ with integer vectors $\mathbf{s}_{j}$ such that $\left|\mathbf{s}_{j}\right| \leq 4 k^{r_{2}}$. We repeat the arguments from the beginning of Section 4.3. Namely, let $(q, p) \in \mathbb{Z}^{2}$ be a pair such that $0<q \leq 4 k^{r_{2}}$ and

$$
|\alpha q+p| \leq \frac{1}{4} k^{-r_{2}}
$$

We choose a pair $(p, q)$ which gives the best approximation. In particular, $p$ and $q$ are mutually prime. Put $\epsilon_{q}:=\alpha+\frac{p}{q}$. We have

$$
k^{-2 r_{2} \mu} \leq\left|\epsilon_{q}\right| \leq \frac{1}{4} q^{-1} k^{-r_{2}} .
$$

We write $\mathbf{s}_{2}$ in the form

$$
\mathbf{s}_{2}=q \mathbf{s}_{2}^{\prime}+\mathbf{s}_{2}^{\prime \prime}
$$

with integer vectors $\mathbf{s}_{2}^{\prime}$ and $\mathbf{s}_{2}^{\prime \prime}, 0 \leq\left(\mathbf{s}_{2}^{\prime \prime}\right)_{j}<q$ for $j=1,2$. Hence, $\left|\left(\mathbf{s}_{2}^{\prime}\right)_{j}\right| \leq 4 k^{r_{2}} / q+1$. It follows

$$
(2 \pi)^{-1} \vec{p}_{\mathbf{m}}=\left(\mathbf{s}_{1}-p \mathbf{s}_{2}^{\prime}\right)+\left(-\frac{p}{q} \mathbf{s}_{2}^{\prime \prime}+\epsilon_{q} \mathbf{s}_{2}^{\prime \prime}\right)+\epsilon_{q} q \mathbf{s}_{2}^{\prime} .
$$


Denote $\mathbf{s}:=\mathbf{s}_{1}-p \mathbf{s}_{2}^{\prime}$. Then $|\mathbf{s}| \leq 8 k^{r_{2}}$. The number of different vectors $\tilde{\mathbf{s}}:=-\frac{p}{q} \mathbf{s}_{2}^{\prime \prime}+\epsilon_{q} \mathbf{s}_{2}^{\prime \prime}$ is not greater than $(2 q)^{2}$. For each fixed pair $\tilde{\mathbf{s}}$, $\mathbf{s}$ we obtain a lattice parameterized by $\mathbf{s}_{2}^{\prime}$. We call this lattice a cluster corresponding to given $\tilde{\mathbf{s}}$, $\mathbf{s}$. Each cluster, obviously, is a square lattice with the step $\epsilon_{q} q$. It contains no more than $\left(9 k^{r_{2}} q^{-1}\right)^{2}$ elements, since $\left|\left(\mathbf{s}_{2}^{\prime}\right)_{j}\right| \leq 4 k^{r_{2}} q^{-1}+1, j=1,2$. The size of each cluster is less than $5\left|\epsilon_{q}\right| k^{r_{2}}$. As before we have the following statements.

Lemma 5.6. Suppose that $\epsilon_{q}$ satisfies the inequality

$$
\left|\epsilon_{q}\right| \leq \frac{1}{64} q^{-1} k^{-r_{2}}
$$

Then, the size of each cluster is less that $\frac{1}{8 q}$. The distance between clusters is greater than $\frac{1}{2 q}$.

Lemma 5.7. The number of vectors $\vec{p}_{\mathbf{m}}$, satisfying the inequalities $\left\|\left|\vec{p}_{\mathbf{m}} \|\right|<2 k^{r_{2}}, p_{\mathbf{m}}<\right.$ $\left|\epsilon_{q}\right| q k^{r_{2} / 3}$, does not exceed $k^{2 r_{2} / 3}$.

Lemma 5.8. Suppose $q$ in the inequality (206) satisfies the estimate $q>k^{2 r_{2} / 3}$. Then, the number of vectors $\vec{p}_{\mathbf{m}},\left\|\left|\vec{p}_{\mathbf{m}} \|\right|<2 k^{r_{2}}\right.$, satisfying the inequality $p_{\mathbf{m}}<k^{-2 r_{2} / 3}$ does not exceed $2^{12} \cdot k^{2 r_{2} / 3}$.

We consider the matrix $H^{(2)}(\vec{\varkappa})=P\left(\gamma r_{1}\right) H(\vec{\varkappa}) P\left(\gamma r_{1}\right)$ where $\vec{\varkappa} \in \mathbb{R}^{2}, P\left(\gamma r_{1}\right)$ is the orthogonal projection corresponding to $\Omega\left(\gamma r_{1}\right)$ (it is a slight abuse of notations, since $H^{(2)}$ in Step II was defined for $\gamma=1)$. Let $\vec{\varkappa}\left(\tau_{1}\right)=\mathbf{b}+\mathbf{a} \tau_{1}, \quad|\mathbf{a}|=1,|\mathbf{b}|<4 k^{\gamma r_{1}}$. We consider $H^{(2)}(\vec{\varkappa})$ as a function of $\tau_{1}$ in the complex $3 k^{-\rho_{1}}$-neighborhood of zero, $\rho_{1}=4 \gamma r_{1}^{\prime}+1+\delta$. We construct the block structure in $H^{(2)}(\vec{\varkappa})$ analogous to that in Step II. The difference is that now we consider any $|\mathbf{b}|<4 k^{\gamma r_{1}}$, not only $\mathbf{b}$ being close to $\vec{\varkappa}^{(1)}(\varphi)$. Here is the construction of the block operator. We call $\mathbf{m} \in \Omega\left(\gamma r_{1}\right)$ resonant in the sense of (72) if

$$
|| \mathbf{b}+\left.\vec{p}_{\mathbf{m}}\right|_{\mathbb{R}} ^{2}-k^{2} \mid<k^{\delta_{*}} .
$$

Next, we introduce $\mathcal{M}_{1}, \mathcal{M}_{2}$ by analogy with Section 3.5.1, here $\mathcal{M}_{1}=\mathcal{M}_{1}(\mathbf{b}), \mathcal{M}_{2}=$ $\mathcal{M}_{2}$ (b). Around each resonant $\mathbf{m} \in \mathcal{M}_{1}$ we construct $k^{\delta}$-blocks. Next, we split $\mathcal{M}_{2}$ into components $\mathcal{M}_{2}^{j}$ and introduce trivial and non-trivial $\mathcal{M}_{2}^{j}$. Further we split each $\mathcal{M}_{2}^{j}$ into $\mathcal{M}_{2}^{j, s}$. Slightly abusing the original Definition 3.19 we say that $\mathcal{M}_{2}^{j, s}$ is weakly resonant if (117) holds with $\mathbf{b}$ instead of $\vec{\varkappa}^{(1)}(\varphi)$. Otherwise, $\mathcal{M}_{2}^{j, s}$ is called strongly resonant. Around each strongly resonant $\mathcal{M}_{2}^{j, s}$ we construct $k^{\delta}$-components and introduce the corresponding projectors (see (145)). Next, we construct a block operator $\tilde{H}^{(1)}(\overrightarrow{\mathcal{L}})$ :

$$
\tilde{H}^{(1)}(\vec{\varkappa})=P H(\vec{\varkappa}) P+H_{0}(\vec{\varkappa})(I-P),
$$

where $P$ is defined in complete analogy with (145).

Next, let $P(\mathbf{m})$ be the projection on the $k^{\delta}$-component containing $\mathbf{m}$. 
Definition 5.9. If

$$
\left\|\left(P(\mathbf{m})\left(H(\mathbf{b})-k^{2}\right) P(\mathbf{m})\right)^{-1}\right\|<k^{4 \gamma r_{1}^{\prime}},
$$

then we call the $k^{\delta}$-cluster effectively non-resonant (cf. (158)) for a given $\mathbf{b}$. Otherwise, it is called effectively resonant.

Definition 5.10. We denote by $J(\mathbf{b})$ the number of effectively resonant clusters.

Lemma 5.11. The resolvent $\left(H^{(2)}(\vec{\varkappa})-k^{2}\right)^{-1}, \vec{\varkappa}=\vec{\varkappa}\left(\tau_{1}\right)$, has no more than $12 J(\mathbf{b})$ poles in the the complex $2 k^{-\rho_{1}}$-neighborhood of $\tau_{1}=0, \rho_{1}=4 \gamma r_{1}^{\prime}+1+\delta$. It satisfies the following estimate in the complex $k^{-\rho_{1}}$-neighborhood of zero:

$$
\left\|\left(H^{(2)}(\vec{\varkappa})-k^{2}\right)^{-1}\right\|<k^{24 \rho_{1}}\left(\frac{2 k^{-\rho_{1}}}{\varepsilon_{0}}\right)^{12 J(\mathbf{b})}, \quad \vec{\varkappa}=\vec{\varkappa}\left(\tau_{1}\right),
$$

where $\varepsilon_{0}=\min \left\{k^{-2 \rho_{1}}, \varepsilon\right\}, \varepsilon$ being the distance to the nearest pole.

Proof. We note that the proof of the lemma is quite similar to that of Theorem 4.1. In both cases we prove the estimates for the resolvent of $H^{(2)}$, considering it as a perturbation of a block operator. There are some technical differences though. Here we have $k^{\gamma r_{1}}$-block around arbitrary $\vec{\varkappa}$, while in Theorem 4.1 we had $k^{r_{1}}$-block around $\vec{\varkappa}^{(1)}(\varphi)$. Here we consider the resolvent as an analytic function of $\tau_{1}$, not $\varphi$. However, the proofs of convergence of perturbation series for the resolvents are quite similar. Though we can't use properties of the non-resonant set $\omega^{(1)}$ now, dependence of the operator on parameter $\tau_{1}$ is simpler than it was on $\varphi$, because diagonal elements of the matrix $H^{(2)}(\vec{\varkappa})$, $\vec{\varkappa}=\vec{\varkappa}\left(\tau_{1}\right)$, are quadratic polynomials of $\tau_{1}$, see (333).

Let us consider blocks of $P H P$. Let $\mathbf{m} \in \mathcal{M}_{1}(\mathbf{b})$. We consider the corresponding $k^{\delta}$-block $P_{\mathbf{m}} H(\vec{\varkappa}) P_{\mathbf{m}}$. Our first goal is to show that each block $\left(P_{\mathbf{m}}\left(H(\vec{\varkappa})-k^{2}\right) P_{\mathbf{m}}\right)^{-1}$, $\mathbf{m} \in \mathcal{M}_{1}(\mathbf{b})$ has no more than 2 poles in a $k^{-\rho_{1}}$-neighborhood of $\tau_{1}=0$ and the estimate

$$
\left\|\left(P_{\mathbf{m}}\left(H(\vec{\varkappa})-k^{2}\right) P_{\mathbf{m}}\right)^{-1}\right\|<k^{4 \rho_{1}}
$$

holds at the distance greater than $k^{-2 \rho_{1}}$ from the poles. Indeed, let $\mathbf{m} \in \mathcal{M}_{1}(\mathbf{b})$, i.e. $\| \vec{\varkappa}(0)+\left.\vec{p}_{\mathbf{m}}\right|_{\mathbb{R}} ^{2}-k^{2} \mid<k^{\delta_{*}}$ and $\| \vec{\varkappa}(0)+\left.\vec{p}_{\mathbf{m}+\mathbf{q}}\right|_{\mathbb{R}} ^{2}-k^{2} \mid>k^{\delta_{*}}$ for $\left\|\left|\vec{p}_{\mathbf{q}} \|\right|<k^{\delta}(\vec{\varkappa}(0)=\mathbf{b})\right.$. We will assume (otherwise the situation becomes trivial) that $\| \vec{\varkappa}(0)+\left.\vec{p}_{\mathbf{m}}\right|_{\mathbb{R}} ^{2}-k^{2} \mid<k^{\delta_{*} / 2}$. Let $\mathbf{D}_{0}$ be such a neighborhood of the corresponding zeros of $\left|\vec{\varkappa}\left(\tau_{1}\right)+\vec{p}_{\mathbf{m}}\right|_{\mathbb{R}}^{2}-k^{2}$ that ||$\vec{\varkappa}\left(\tau_{1}\right)+\left.\vec{p}_{\mathbf{m}}\right|_{\mathbb{R}} ^{2}-k^{2} \mid=k^{\delta_{*}-\delta}$ on $\partial \mathbf{D}_{0}$. Since $\left|\vec{\varkappa}\left(\tau_{1}\right)+\vec{p}_{\mathbf{m}}\right|_{\mathbb{R}}^{2}$ is a quadratic polynomial in $\tau_{1}$ with the main coefficient 1 , the set $\mathbf{D}_{0}$, obviously, belongs to the $k^{\left(\delta_{*}-\delta\right) / 2}$-neighborhood of the zeros. We consider only a connected component of $\mathbf{D}_{0}$, which intersects $k^{\delta_{*}-3 \delta_{-}}$ neighborhood of $\tau_{1}=0$ (with a slight abuse of notations we still denote it $\mathbf{D}_{0}$ ). Clearly,

$$
\left(\left|\vec{\varkappa}\left(\tau_{1}\right)+\vec{p}_{\mathbf{m}+\mathbf{q}}\right|_{\mathbb{R}}^{2}-\left|\vec{\varkappa}\left(\tau_{1}\right)+\vec{p}_{\mathbf{m}}\right|_{\mathbb{R}}^{2}\right)-\left(\left|\vec{\varkappa}(0)+\vec{p}_{\mathbf{m}+\mathbf{q}}\right|_{\mathbb{R}}^{2}-\left|\vec{\varkappa}(0)+\vec{p}_{\mathbf{m}}\right|_{\mathbb{R}}^{2}\right)=2 \tau_{1}\left(\mathbf{a}, \vec{p}_{\mathbf{q}}\right) .
$$

Considering that $\left|\tau_{1}\right|<k^{\delta_{*}-2 \delta}$, we obtain:

$$
|| \vec{\varkappa}\left(\tau_{1}\right)+\left.\vec{p}_{\mathbf{m}+\mathbf{q}}\right|_{\mathbb{R}} ^{2}-k^{2} \mid \geq \frac{1}{2} k^{\delta_{*}} \text { on } \partial \mathbf{D}_{0}
$$


Using simple perturbation arguments, we see that $\left(P_{\mathbf{m}}\left(H-k^{2}\right) P_{\mathbf{m}}\right)^{-1}$ has no more than 2 poles in $\mathbf{D}_{0}$ and

$$
\left\|\left(P_{\mathbf{m}}\left(H(\vec{\varkappa})-k^{2}\right) P_{\mathbf{m}}\right)^{-1}\right\|<2 k^{-\delta_{*}+\delta} \text { on } \partial \mathbf{D}_{0} .
$$

Now, shrinking the neighborhood around poles we prove (336).

Next, we consider the case of a trivial $\mathcal{M}_{2}^{j}$. Each such $\mathcal{M}_{2}^{j, s}$ consists just from one point $\mathbf{m}$. Assume that $\mathcal{M}_{2}^{j, s}$ is strongly resonant, i.e., $\mathbf{m}:|| \vec{\varkappa}(0)+\left.\vec{p}_{\mathbf{m}}\right|_{\mathbb{R}} ^{2}-k^{2} \mid<k^{-214 \mu \delta}$. Then, (cf. Lemma 3.20) we have at most 2 such $\mathbf{m}$-s. Choosing a neighborhood of the size $k^{-300 \mu \delta}$ around zeros of the the corresponding quadratic polynomials (see the case $\mathbf{m} \in \mathcal{M}_{1}$ ), we can prove the result similar to Lemma 3.24. In particular, the resolvent of the corresponding $k^{\delta}$-cluster has no more than four poles $\tau_{1}$ in the $4 k^{-300 \mu \delta}$ neighborhood of zero and estimate analogous to (336) holds (with $8 \rho_{1}$ instead of $4 \rho_{1}$ in the r.h.s). If $\mathcal{M}_{2}^{j, s}$ is just weakly resonant, than there are no poles of the resolvent in the $k^{-300 \mu \delta}$ of zero.

It remains to consider a non-trivial case. We start with the model operator $H^{j, s}\left(\tau_{1}\right)$. and assume that it is strongly resonant:

$$
\left\|\left(H^{j, s}(\mathbf{b})-k^{2}\right)^{-1}\right\|>k^{214 \mu \delta} .
$$

Considering as in the proof of Lemma 3.18, we introduce eigenvalues $\tilde{\lambda}_{n}\left(t_{\mathbf{q}}\right)=\lambda_{n}\left(t_{\mathbf{q}}\right)-$ $\left(t_{\mathbf{q}}^{\perp}\right)^{2}\left(\tau_{1}\right)$ and corresponding eigenvalues of the periodic one-dimensional operator $\tilde{\lambda}_{n}^{\text {per }}\left(t_{\mathbf{q}}\right)$, here and below, $t_{\mathbf{q}}=\left(\mathbf{b}+\vec{p}_{\mathbf{m}}, \vec{\nu}_{\mathbf{q}}\right)+\tau_{1}\left(\mathbf{a}, \vec{\nu}_{\mathbf{q}}\right), t_{\mathbf{q}}^{\perp}=\left(\mathbf{b}+\vec{p}_{\mathbf{m}}, \vec{\nu}_{\mathbf{q}}^{\perp}\right)+\tau_{1}\left(\mathbf{a}, \vec{\nu}_{\mathbf{q}}^{\perp}\right)$, m being the central point of $\mathcal{M}_{2}^{j, s}$, and $\left|\tau_{1}\right|<\sigma_{\text {large }}, \sigma_{\text {large }}=\pi p_{\mathbf{q}} / 2$. We have $\left|\tilde{\lambda}_{n}\left(t_{\mathbf{q}}\right)\right| \leq \frac{1}{8} k^{\delta_{*}}$, $\tilde{\lambda}_{n}^{\text {per }}\left(t_{\mathbf{q}}\right)=\tilde{\lambda}_{n}\left(t_{\mathbf{q}}\right)+O\left(k^{-\frac{\delta_{*}}{C(Q)} k^{\delta_{*} / 2}}\right)$. Assume, first, that $\left|\tilde{\lambda}_{n}^{\text {per }}\left(t_{\mathbf{q}}\right)\right|>\Lambda$ with sufficiently large $\Lambda$ to be fixed later. Only two eigenvalues, say, with indices $i_{0}$ and $i_{0}+1$ can be close to each other. Now, the arguments are somewhat similar to those from the proof of Lemma 4.14, see also Appendix 5. Indeed, let us consider the expression:

$$
D_{i_{0}}:=\left(\tilde{\lambda}_{i_{0}}\left(\tau_{1}\right)+\left(t_{\mathbf{q}}^{\perp}\right)^{2}-k^{2}\right)\left(\tilde{\lambda}_{i_{0}+1}\left(\tau_{1}\right)+\left(t_{\mathbf{q}}^{\perp}\right)^{2}-k^{2}\right) .
$$

Since $i_{0}$ is big enough, all other eigenvalues are sufficiently far away and perturbation arguments work. Unlike each individual factor in the r.h.s. of (337), $D_{i_{0}}$ is analytic in the neighborhood of $\tau_{1}=0$ with the radius of analyticity $\sigma_{\text {large }}$. We assume that both factors in $D_{i_{0}}$ are close to zero, i.e. $\left|\tilde{\lambda}_{i_{0}}-\tilde{\lambda}_{i_{0}+1}\right| \leq \Lambda^{-1 / 2}$, otherwise, only one factor is needed which makes arguments even simpler. If $\Lambda=\Lambda(V)$ is large enough we can apply standard perturbative arguments (see Appendix 5 for details) to compare $D_{i_{0}}$ with the same expression for $V=0$ which we denote by $D_{i_{0}, 0}$. Obviously, $D_{i_{0}, 0}$ is a polynomial of order four with respect to $\tau_{1}$ with the main coefficient 1 . We consider $\sigma_{\text {large }} / 100$ neighborhood of each zero and denote the union of these neighborhoods (intersecting the $k^{-41 \mu \delta}$-neighborhood of $\left.\tau_{1}=0\right)$ by $T_{0}$. By definition, $T_{0}$ consists of no more than 4 discs. Without loss of generality we may assume that $T_{0}$ is in the domain of analyticity of $D_{i_{0}}$ 
(otherwise, it means that a particular zero of $D_{i_{0}, 0}$ and $D_{i_{0}}$ is far away from $\tau_{1}=0$ and is not of our interest). We have

$$
\left|D_{i_{0}, 0}\left(\tau_{1}\right)\right|>\left(\sigma_{\text {large }} / 100\right)^{4} \quad \text { on } \partial T_{0} .
$$

Now, we choose $\Lambda=\Lambda(V)$ as described in Appendix 5. By perturbation and Rouché's Theorem, $D_{i_{0}}$ has no more than 4 zeros in $T_{0}$ (obviously, even in twice more narrow neighborhood) and

$$
\left\|\left(H^{j, s}-k^{2}\right)^{-1}\right\| \leq 2\left(100 / \sigma_{\text {large }}\right)^{4} \quad \text { on } \partial T_{0} .
$$

Let $\left|\tilde{\lambda}_{n}^{\text {per }}\left(t_{\mathbf{q}}\right)\right| \leq \Lambda(V)$. Put

$$
C_{*}=C_{*}(V, \Lambda):=\Lambda+\max _{s, n:\left|\lambda_{n}(s)\right| \leq \Lambda}\left\{\left|\lambda_{n}^{\prime}(s)\right|+\left|\lambda_{n}^{\prime \prime}(s)\right|+\left|\lambda_{n}^{\prime \prime \prime}(s)\right|\right\} .
$$

If $\left|\left(\mathbf{a}, \vec{\nu}_{\mathbf{q}}^{\perp}\right)\left(\mathbf{b}+\vec{p}_{\mathbf{m}}, \vec{\nu}_{\mathbf{q}}^{\perp}\right)\right| \geq 2 C_{*}$, then

$$
\left|\frac{d}{d \tau_{1}}\left(\tilde{\lambda}_{n}^{\text {per }}\left(t_{\mathbf{q}}\right)+\left(t_{\mathbf{q}}^{\perp}\right)^{2}\left(\tau_{1}\right)\right)\right| \geq C_{*}
$$

in a complex neighborhood $\sigma, \sigma=\sigma(V, \Lambda)$, of $\tau_{1}=0$. This means that $\tilde{\lambda}_{n}^{\text {per }}\left(t_{\mathbf{q}}\right)+$ $\left(t_{\mathbf{q}}^{\perp}\right)^{2}\left(\tau_{1}\right)-k^{2}$ has no more than one zero in this neighborhood and it can happen only for a real $\tau_{1}$. Estimate of the type (339) (2 instead of 4 and $\sigma$ instead of $\sigma_{\text {large }}$ in the r.h.s) holds on the boundary of the neighborhood. If $\left|\left(\mathbf{a}, \vec{\nu}_{\mathbf{q}}^{\perp}\right)\left(\mathbf{b}+\vec{p}_{\mathbf{m}}, \vec{\nu}_{\mathbf{q}}^{\perp}\right)\right|<2 C_{*}$, then

$$
\begin{aligned}
\frac{d^{2}}{d \tau_{1}^{2}}\left(\tilde{\lambda}_{n}^{\text {per }}\left(t_{\mathbf{q}}\right)+\left(t_{\mathbf{q}}^{\perp}\right)^{2}\left(\tau_{1}\right)\right) & =\frac{d^{2}}{d t_{\mathbf{q}}^{2}} \tilde{\lambda}_{n}^{p e r}\left(t_{\mathbf{q}}\right)+O_{\Lambda, V}\left(k^{-2}\right), \\
\left|\frac{d^{3}}{d \tau_{1}^{3}}\left(\tilde{\lambda}_{n}^{p e r}\left(t_{\mathbf{q}}\right)+\left(t_{\mathbf{q}}^{\perp}\right)^{2}\left(\tau_{1}\right)\right)\right| & =\left|\frac{d^{3}}{d t_{\mathbf{q}}^{3}} \tilde{\lambda}_{n}^{p e r}\left(t_{\mathbf{q}}\right)\right|+O_{\Lambda, V}\left(k^{-2}\right) .
\end{aligned}
$$

It follows from the properties of the one-dimensional periodic Schrödinger operator (see e.g. 43]) that

$$
\left|\frac{d^{2}}{d t_{\mathbf{q}}^{2}} \tilde{\lambda}_{n}^{p e r}\left(t_{\mathbf{q}}\right)\right|+\left|\frac{d^{3}}{d t_{\mathbf{q}}^{3}} \tilde{\lambda}_{n}^{\text {per }}\left(t_{\mathbf{q}}\right)\right|>c(V, \Lambda)>0 .
$$

This means that $\tilde{\lambda}_{n}^{\text {per }}\left(t_{\mathbf{q}}\right)+\left(t_{\mathbf{q}}^{\perp}\right)^{2}-k^{2}$ has no more than three zeros $\tau_{1}$ in a $\sigma$-neighborhood (for details see Appendix 10). Thus we can apply again the same arguments as above to prove an estimate similar to (339) with 6 instead of 4. Next, as in Lemma 3.20 there can be no more than 2 strongly resonant sets $\mathcal{M}_{2}^{j, s}$ in a cluster of $k^{\delta}$-blocks. Using (339) we can proceed as in the proof of Lemma 3.25 and, in particular, prove the analog of (336) for an effectively strongly resonant non-trivial cluster (with $24 \rho_{1}$ instead of $4 \rho_{1}$ ). If $\mathcal{M}_{2}^{j, s}$ is a non-trivial weakly resonant cluster disjoint from any strongly resonant cluster, it does not generate any poles. 
We proved that the resolvent $\left(\tilde{H}^{(1)}(\vec{\varkappa})-k^{2}\right)^{-1}$ has no more than $12 J(\mathbf{b})$ poles $\tau_{1 j}$ in the complex $k^{-1-40 \mu \delta}$-neighborhood of $\tau_{1}=0$ and satisfies the estimate

$$
\left\|\left(\tilde{H}^{(1)}(\vec{\varkappa})-k^{2}\right)^{-1}\right\|<\frac{1}{4} k^{24 \rho_{1}}
$$

at the distance greater than $k^{-2 \rho_{1}}$ from the poles. Let us consider the union of $k^{-2 \rho_{1}}$ neighborhoods of these poles. It may consist from several connected components. We are interested only in those intersecting with the $2 k^{-\rho_{1}}$-disk around $\tau_{1}=0$. We denote their union by $\mathbf{D}$. Using a rough estimate $J(\vec{\varkappa})<k^{4 \gamma r_{1}}$ gives that $\mathbf{D}$ belongs to the $3 k^{-\rho_{1}}$-neighborhood of zero. Thus, (341) holds outside D. Now, considering as in the proof of Theorem 4.1 (see (178) -(190) ), we can show that the perturbation series for the resolvent $\left(H^{(2)}(\vec{\varkappa})-k^{2}\right)^{-1}$ with respect to $\left(\tilde{H}^{(1)}(\vec{\varkappa})-k^{2}\right)^{-1}$ converges on the boundary of $\mathrm{D}$ and

$$
\left\|\left(H^{(2)}(\vec{\varkappa})-k^{2}\right)^{-1}\right\|<k^{24 \rho_{1}}
$$

outside $\mathbf{D}$; the resolvent has no more than $12 J(\vec{\varkappa})$ poles in $\mathbf{D}$. Using again the maximum principle we obtain (335).

Note that each connected component of $\mathcal{S}^{(2)}(k, \xi)$, see (327), is bounded by the curves $D\left(\vec{\varkappa}, k^{2} \pm k^{-\xi}\right)=0$, where $D(\vec{\varkappa}, \lambda)=\operatorname{det}\left(H^{(2)}(\vec{\varkappa})-\lambda\right)$.

Lemma 5.12. Let $\mathbf{l}$ be a segment of a straight line in $\mathbb{R}^{2}$,

$$
\mathbf{l}:=\left\{\vec{\varkappa}=\mathbf{a} \tau_{1}+\mathbf{b}, \tau_{1} \in(0, \eta)\right\}, \quad|\mathbf{a}|=1,|\mathbf{b}|<4 k^{\gamma r_{1}}, \quad 0<\eta<k^{-5 \gamma r_{1}^{\prime}} .
$$

Suppose both ends of $\mathbf{l}$ belong to a connected component of $\mathcal{S}^{(2)}(k, \xi)$. If $\xi$ is sufficiently large, namely, $\xi \geq 48 J(\mathbf{b}) \ln _{k} \frac{1}{\eta}$, then, there is an inner part $\mathbf{l}^{\prime}$ of the segment, which is not in $\mathcal{S}^{(2)}(k, \xi)$.

Corollary 5.13. Let $\vec{\varkappa} \in \mathcal{S}^{(2)}(k, \xi)$ and $\frac{\xi}{48 J(\vec{\varkappa})}>10 \gamma r_{1}^{\prime}$. Then the distance from $\vec{\varkappa}$ to the boundary of $\mathcal{S}^{(2)}(k, \xi)$ is less than $k^{-\frac{\xi}{48 J(\vec{x})}}$.

Proof of the corollary. Let us consider a segment of the length $\eta=k^{-\frac{\xi}{48 J(\vec{\varkappa})}}$ starting at $\vec{\varkappa}$. By the statement of the lemma it intersects a boundary $D\left(\vec{\varkappa}, k^{2} \pm k^{-\xi}\right)=0$.

Proof. Choose $\varepsilon=\eta^{2}$ in (335). Using the hypothesis of the lemma, we obtain that the right-hand side of (335) is less than $k^{\xi}$ outside the discs of radius $\varepsilon$ around the poles of the resolvent. Let us estimate the total size (sum of the sizes) of the discs. Indeed, the size of each disc is $2 \varepsilon$ and the number of discs is, obviously, less $16 k^{4 \gamma r_{1}}$. Therefore, the total size admits the estimate from above: $32 \varepsilon k^{4 \gamma r_{1}}<<\eta$, since $\eta<k^{-5 \gamma r_{1}^{\prime}}$. This means there is a part $\mathbf{l}^{\prime}$ of $\mathbf{l}$ outside these discs. By (335), this part is outside $\mathcal{S}^{(2)}(k, \xi)$, when $\xi$ is as described in the statement of the lemma.

Let $\vec{\varkappa}_{0} \in \mathbb{R}^{2}$ be fixed and $\mathcal{N}\left(k, r_{2}, \vec{\varkappa}_{0}, J_{0}\right)$ be the following subset of the lattice $\vec{\varkappa}_{0}+\vec{p}_{\mathbf{n}}$, $\mathbf{n} \in \Omega\left(r_{2}\right):$

$$
\mathcal{N}\left(k, r_{2}, \vec{\varkappa}_{0}, J_{0}\right)=\left\{\vec{\varkappa}_{0}+\vec{p}_{\mathbf{n}}: \mathbf{n} \in \Omega\left(r_{2}\right): J\left(\vec{\varkappa}_{0}+\vec{p}_{\mathbf{n}}\right) \leq J_{0}\right\}
$$


$J$ being defined by Definition 5.10 , Thus, $\mathcal{N}$ includes only such $\mathbf{n}$ that the surrounding $k^{\gamma r_{1}}$ - block contains less than $J_{0}$ effectively resonant $k^{\delta}$-clusters. Let $N\left(k, r_{2}, \vec{\varkappa}_{0}, J_{0}, \xi\right)$ be the number of points $\vec{\varkappa}_{0}+\vec{p}_{\mathbf{n}}$ in $\mathcal{S}^{(2)}(k, \xi) \cap \mathcal{N}\left(k, r_{2}, \vec{\varkappa}_{0}, J_{0}\right)$.

Lemma 5.14. If $r_{2}>10 \gamma r_{1}^{\prime}$ and $\xi>96 \mu r_{2} J_{0}$, then

$$
N\left(k, r_{2}, \vec{\varkappa}_{0}, J_{0}, \xi\right) \leq k^{\frac{2}{3} r_{2}+43 \gamma r_{1}} .
$$

Proof. Let us call a subset $\tilde{\mathcal{S}}$ of $\mathcal{S}^{(2)}(k, \xi)$ elementary if it can be described by a formula of the type:

$$
\tilde{\mathcal{S}}:=\left\{\vec{\varkappa}: a<\varkappa_{1}<b, f_{1}\left(\varkappa_{1}\right)<\varkappa_{2}<f_{2}\left(\varkappa_{1}\right)\right\}
$$

where the curves $\varkappa_{2}=f_{i}\left(\varkappa_{1}\right), i=1,2$, belong to the boundary of $\mathcal{S}^{(2)}(k, \xi)$, have the lengths less than 1 , functions $f_{i}\left(\varkappa_{1}\right)$ are monotone, continuously differentiable and have no inflection points. We assume that the boundaries $\varkappa_{2}=f_{i}\left(\varkappa_{1}\right)$ are parameterized by $\varkappa_{1}$ for definiteness. The set where $\varkappa_{1}=f_{i}\left(\varkappa_{2}\right), a<\varkappa_{2}<b$, is completely analogous.

Next, we show that the number of points in $\tilde{\mathcal{S}} \cap \mathcal{N}\left(k, r_{2}, \vec{\varkappa}_{0}, J_{0}\right)$ does not exceed $2^{14} k^{\frac{2}{3} r_{2}}$. Indeed, let us consider a segment $\vec{p}_{\mathbf{n}-\mathbf{n}^{\prime}}$ between two points $\vec{\varkappa}_{0}+\vec{p}_{\mathbf{n}}$ and $\vec{\varkappa}_{0}+\vec{p}_{\mathbf{n}^{\prime}}$ in $\tilde{\mathcal{S}}$. Obviously, $\left\|\left|\vec{p}_{\mathbf{n}-\mathbf{n}^{\prime}} \|\right|<2 k^{r_{2}}\right.$ and $p_{\mathbf{n}-\mathbf{n}^{\prime}}>k^{-\mu r_{2}}$. The direction of the segment cannot be parallel to the axis $\varkappa_{2}$ by Corollary 5.13. We enumerate the points $\vec{\varkappa}_{0}+\vec{p}_{\mathbf{n}} \in \tilde{\mathcal{S}} \cap$ $\mathcal{N}\left(k, r_{2}, \vec{\varkappa}_{0}, J_{0}\right)$ in the order of the increasing first coordinate and connect subsequent points by segments. Consider all segments with the length greater or equal to $\frac{1}{64} k^{-\frac{2 r_{2}}{3}}$. The number of such segments does not exceed $128 k^{\frac{2 r_{2}}{3}}$, since they are much longer than the width of $\tilde{\mathcal{S}}$ (Corollary 5.13). It remains to estimate the number of segments with the length less than $\frac{1}{64} k^{-\frac{2 r_{2}}{3}}$.

First, we prove that no more than two segments $\vec{p}_{\mathbf{n}_{1}-\mathbf{n}_{1}^{\prime}}, \vec{p}_{\mathbf{n}_{2}-\mathbf{n}_{2}^{\prime}}$ can be parallel to each other, if they are in the same elementary component $\tilde{\mathcal{S}}$. Indeed, both ends of $\vec{p}_{\mathbf{n}_{1}-\mathbf{n}_{1}^{\prime}}$ are in $\tilde{\mathcal{S}}$. By the previous lemma there is a piece of the segment which is not in $\tilde{\mathcal{S}}$ (we notice that now we use the lemma for $k^{-\mu r_{2}}<\eta<\frac{1}{64} k^{-\frac{2 r_{2}}{3}}$ ). Hence, the segment intersects one of the curves $\varkappa_{2}=f_{i}\left(\varkappa_{1}\right)$ twice. It follows, that there is a point on the curve, where the curve is parallel to the segment. Suppose another segment $\vec{p}_{\mathbf{n}_{2}-\mathbf{n}_{2}^{\prime}}$ intersects the same curve. Then, there is a point on the curve, where the curve is parallel to the second segment. Since the curve is concave, it can not be the case. Therefore, $\vec{p}_{\mathbf{n}_{2}-\mathbf{n}_{2}^{\prime}}$ intersects another curve. It follows that no more than two segments $\vec{p}_{\mathbf{n}_{1}-\mathbf{n}_{1}^{\prime}}, \vec{p}_{\mathbf{n}_{2}-\mathbf{n}_{2}^{\prime}}$ can be parallel to each other, if they are in the same elementary component $\tilde{\mathcal{S}}$.

To finish the proof of the lemma we consider two cases. Suppose $q$ in the inequality (328) satisfies the estimate $q>k^{2 r_{2} / 3}$. Then, by Lemma 5.8, the number of vectors $\vec{p}_{\mathbf{n}}$, $\left\|\left|\vec{p}_{\mathbf{n}} \|\right|<2 k^{r_{2}}\right.$, satisfying the inequality $p_{\mathbf{n}}<\frac{1}{64} k^{-2 r_{2} / 3}$ does not exceed $2^{12} k^{2 r_{2} / 3}$. Since each of them can be used only twice, the total number of short segments does not exceed $2^{13} k^{2 r_{2} / 3}$.

Let $q \leq k^{2 r_{2} / 3}$. If $\left|\epsilon_{q}\right|>\frac{1}{64} q^{-1} k^{-r_{2}}$. Then, obviously, $\frac{1}{64} k^{-2 r_{2} / 3}<\left|\epsilon_{q}\right| q k^{r_{2} / 3}$. Applying Lemma 5.7, we obtain that the number of segments with the length less than $\frac{1}{64} k^{-2 r_{2} / 3}$ 
is less than $k^{2 r_{2} / 3}$. Since each of them can be used only twice, the total number of short segments does not exceed $2 k^{2 r_{2} / 3}$. It remains to consider the case $q \leq k^{2 r_{2} / 3}$, $\left|\epsilon_{q}\right| \leq \frac{1}{64} q^{-1} k^{-r_{2}}$. By Lemma 5.6, clusters are well separated. Considering that the distance between clusters is greater than $\frac{1}{2 q}$ and the size of each cluster is less than $\frac{1}{8 q}$, we obtain that no more than $8 q$ clusters can intersect $\tilde{\mathcal{S}}$. Indeed, the distance between two clusters is greater than $\frac{1}{2 q}$. By Corollary 5.13 , the set $\tilde{\mathcal{S}}$ belongs to the $k^{-\frac{\xi}{48 J_{0}}}$-neighborhood of each curve $\varkappa_{2}=f_{i}\left(\varkappa_{1}\right), i=1,2$. Using the hypothesis of the lemma we easily get that the size of the neighborhood is $o\left(q^{-1}\right)$. If a cluster intersects $\tilde{\mathcal{S}}$, its $\frac{1}{4 q}$-neighborhood intersects both curves $\varkappa_{2}=f_{i}\left(\varkappa_{1}\right), i=1,2$. Since the distance between clusters is greater than $\frac{1}{2 q}$, the distance along the curve between its intersection with $\frac{1}{4 q}$-neighborhoods of different clusters is greater than $\frac{1}{4 q}$. Considering that the lengths of the curves is less than 1 , we obtain that no more than $8 q$ clusters can intersect $\tilde{\mathcal{S}}$. Next, the segments with the length less than $\frac{1}{2} k^{-2 r_{2} / 3}$ cannot connect different clusters, since the distance between clusters is greater than $\frac{1}{2 q} \geq \frac{1}{2} k^{-2 r_{2} / 3}$. Therefore, any segment of the length less than $\frac{1}{2} k^{-2 r_{2} / 3}$ is inside one cluster. The part of the shorter curve inside the clusters has the length $L_{i n}$ which is less than the double size of a cluster $10\left|\epsilon_{q}\right| k^{r_{2}}$ (the curve is concave) multiplied by the number of clusters $8 q$, i.e., $L_{i n}<80\left|\epsilon_{q}\right| q k^{r_{2}}$. If we consider the segments with the length greater than $\left|\epsilon_{q}\right| q k^{r_{2} / 3}$, then the number of such segments is less than $L_{\text {in }} /\left|\epsilon_{q}\right| q k^{r_{2} / 3}$, i.e., it is less than $80 k^{2 r_{2} / 3}$. By Lemma 5.7, the total number of segments of the length less than $\left|\epsilon_{q}\right| q k^{r_{2} / 3}$ is less than $k^{2 r_{2} / 3}$. Each of them can be used only twice. Thus, the total number of segments is less than $162 k^{2 r_{2} / 3}$.

We proved that the number of segments in $\tilde{\mathcal{S}}$ does not exceed $2^{14} k^{2 r_{2} / 3}$. Therefore, the number of points in $\tilde{\mathcal{S}} \cap \mathcal{N}\left(k, r_{2}, \vec{\varkappa}_{0}, J_{0}\right)$ does not exceed $2^{14} k^{\frac{2}{3} r_{2}}+1$. Considering that $k^{\gamma r_{1}}>2^{15}$, we obtain that the number of points inside $\tilde{\mathcal{S}} \cap \mathcal{N}\left(k, r_{2}, \vec{\varkappa}_{0}, J_{0}\right)$ does not exceed $k^{\frac{2}{3} r_{2}+\gamma r_{1}}$.

If we show that $\mathcal{S}^{(2)}(k, \xi)$ is the union of no more than $k^{42 \gamma r_{1}}$ elementary components $\tilde{\mathcal{S}}$, then estimate (343) easily follows. Indeed, let us consider the boundary of $\mathcal{S}^{(2)}(k, \xi)$. It is described by curves $D\left(\vec{\varkappa}, k^{2} \pm k^{-\xi}\right)=0, \vec{\varkappa} \in \mathbb{R}^{2}$. We break each curve into elementary components as described in Appendix 8. By Lemma 10.12 the number of such pieces is less than $k^{17 \gamma r_{1}}$. With each elementary piece of the boundary we associate the part of the adjacent connected component of $\mathcal{S}^{(2)}(k, \xi)$, which is in the $k^{-\frac{\xi}{48 J_{0}}}$-neighborhood of the elementary piece. By Corollary 5.13, every point in $\mathcal{S}^{(2)}(k, \xi)$ belongs to such a component, some components overlapping. Let us consider one of these components $\hat{\mathcal{S}}$. By construction, it is adjacent to a boundary elementary component, which can be described in the form $\varkappa_{1}=f_{1}\left(\varkappa_{2}\right)$ or $\varkappa_{2}=f_{1}\left(\varkappa_{1}\right)$. Let us assume for definiteness that it is described by the formula $\varkappa_{2}=f_{1}\left(\varkappa_{1}\right)$. By Corollary 5.13, there is another boundary (described by $\left.\varkappa_{2}=f_{2}\left(\varkappa_{1}\right)\right)$ of $\hat{\mathcal{S}}$ in the $k^{-\frac{\xi}{48 J_{0}}}$-neighborhood of $\varkappa_{2}=f_{1}\left(\varkappa_{1}\right)$. It also can be split into no more than $k^{17 \gamma r_{1}}$ elementary components. Further, each elementary component contains no more than $k^{8 \gamma r_{1}}$ points $\vec{\varkappa}: D\left(\vec{\varkappa}, k^{2}+k^{-\xi}\right)=D\left(\vec{\varkappa}, k^{2}-k^{-\xi}\right)=0$, unless the last equality 
is an identity on this component (Bezout Theorem). We use these points to break each elementary component into at most $k^{8 \gamma r_{1}}$ parts. Correspondingly, we split the set $\hat{\mathcal{S}}$ by lines $\varkappa_{1}=C$ into at most $k^{25 \gamma r_{1}}$ components $\tilde{\mathcal{S}}$. The second boundary of $\tilde{\mathcal{S}}$ also can be parameterized by $\varkappa_{2}$, since $D_{\varkappa_{2}} \neq 0$ on an elementary component of the boundary. By the definition of an elementary component of the boundary (Appendix 8), both functions $\varkappa_{2}=f_{i}\left(\varkappa_{1}\right)$ are monotone, continuously differentiable and don't have inflection points, the length of the corresponding curves being less than 1. Moreover, neither boundary contains intersections with other pieces of the boundary of $\mathcal{S}^{(2)}(k, \xi)$. Thus, $\mathcal{S}^{(2)}(k, \xi)$ is the union of at most $k^{42 \gamma r_{1}}$ elementary components $\tilde{\mathcal{S}}$, each being bounded by lines $\varkappa_{i}=a, b$ and elementary pieces of the boundary of $\mathcal{S}^{(2)}(k, \xi)$, which do not intersect with other pieces of the boundary of $\mathcal{S}^{(2)}(k, \xi)$.

\subsubsection{Model Operator for Step IV}

Let $r_{3}>r_{2}$. We repeat for $r_{3}$ the construction from the subsection 4.4.1, which was done for arbitrary $r_{2}>r_{1}$. In particular, we introduce the corresponding sets $\mathcal{M}_{2}^{\text {weak }}\left(r_{3}, \varphi_{0}\right)$, $\mathcal{M}_{2}^{s t r}\left(r_{3}, \varphi_{0}\right), \mathcal{M}_{2, t w}\left(r_{3}, \varphi_{0}\right)$. Let $\mathbf{m} \in \Omega\left(r_{3}\right)$. We denote the $k^{\gamma r_{1}}$-component containing $\mathbf{m}$ by $\tilde{\Pi}(\mathbf{m})$ and the corresponding projector by $\tilde{P}(\mathbf{m})$. For $\mathbf{m}$ belonging to the same $k^{\gamma r_{1}}$-component, $\tilde{\Pi}(\mathbf{m})$ and $\tilde{P}(\mathbf{m})$ are the same. Put

$$
\mathcal{M}^{(3)}:=\mathcal{M}^{(3)}\left(\varphi_{0}, r_{3}\right)=\left\{\mathbf{m} \in \mathcal{M}^{(2)}\left(\varphi_{0}, r_{3}\right) \cup \Omega_{s}^{(2)}\left(r_{3}\right) \backslash \mathcal{M}_{2}^{\text {weak }}\left(r_{3}, \varphi_{0}\right): \varphi_{0} \in \mathcal{O}_{\mathbf{m}}^{(3)}\left(r_{2}^{\prime}, 1\right)\right\},
$$

where $\Omega_{s}^{(2)}\left(r_{3}\right)$ is the extension of $\Omega_{s}^{(2)}\left(r_{2}\right)$ to $\Omega\left(r_{3}\right)$,

$$
\Omega_{s}^{(2)}\left(r_{3}\right)=\left\{\mathbf{m} \in \Omega\left(r_{3}\right), 0<p_{\mathbf{m}} \leq k^{-5 r_{1}^{\prime}}\right\},
$$

$\mathcal{O}_{\mathbf{m}}^{(3)}\left(r_{2}^{\prime}, \tau\right)$ is the union of the disks of the radius $\tau k^{-r_{2}^{\prime}}$ with the centers at poles of the resolvent $\left(\tilde{P}(\mathbf{m})\left(H\left(\vec{\varkappa}^{(2)}(\varphi)\right)-k^{2} I\right) \tilde{P}(\mathbf{m})\right)^{-1}$ in the $k^{-44 r_{1}^{\prime}-2-\delta}$-neighborhood of $\varphi_{0}$. (Here $\mathcal{M}^{(2)}\left(\varphi_{0}, r_{3}\right)$ is defined as in (251) with $r_{3}$ instead of $\left.r_{2}\right)$. For $\mathbf{m}$ belonging to the same $k^{\gamma r_{1}}$-component, the sets $\mathcal{O}_{\mathbf{m}}^{(3)}\left(r_{2}^{\prime}, \tau\right)$ are identical. We say that $\mathbf{m} \in \mathcal{M}^{(3)}$ is $k^{\gamma r_{1}}$-resonant. The corresponding $k^{\gamma r_{1}}$-clusters we call resonant too.

Let $\varphi_{0} \in \omega^{(3)}(k, \delta, 1)$. By construction of the non-resonant set $\omega^{(3)}(k, \delta, 1)$, we have $\mathcal{M}^{(3)} \cap \Omega\left(r_{2}\right)=\emptyset$.

Further we use the property of the set $\mathcal{M}^{(3)}$ formulated in the next lemma which is an analogue of the Lemma 4.18 .

Lemma 5.15. Let $r_{2}^{\prime}>2 k^{\left(\gamma+\delta_{0}\right) 10^{-4} r_{1}-2 \delta_{*}}$. Let $1 / 20<\gamma^{\prime}<20, \mathbf{m}_{0} \in \Omega\left(r_{3}\right)$ and $\Pi_{\mathbf{m}_{0}}$ be the $k^{\gamma^{\prime} r_{2}}$-neighborhood (in $\||\cdot|\| \mid$-norm) of $\mathbf{m}_{0}$. Then the set $\Pi_{\mathbf{m}_{0}}$ contains less than $k^{\frac{2}{3} \gamma^{\prime} r_{2}+50 \gamma r_{1}}$ elements of $\mathcal{M}^{(3)}$.

Proof. First, we notice that the condition $r_{2}^{\prime}>2 k^{\left(\gamma+\delta_{0}\right) 10^{-4} r_{1}-2 \delta_{*}}$ is consistent with the restriction $r_{2}^{\prime}<k^{\delta_{0} r_{1}-4}$ in (278). If $\mathbf{m} \in \mathcal{M}^{(3)}$, then there is a $\varphi_{*}$ such that $\left|\varphi_{*}-\varphi_{0}\right|<k^{-r_{2}^{\prime}}$ and

$$
\operatorname{det}\left(\tilde{P}(\mathbf{m})\left(H\left(\vec{\varkappa}^{(2)}\left(\varphi_{*}\right)\right)-k^{2} I\right) \tilde{P}(\mathbf{m})\right)=0,
$$


where $\tilde{P}(\mathbf{m})$ is the projection corresponding to the $k^{r_{1}}$-cluster $\tilde{\Pi}(\mathbf{m})$, which includes $\mathbf{m}$. The cluster $\tilde{\Pi}(\mathbf{m})$ can be simple, white, grey or black. Since $\varphi_{0}$ is close to $\varphi_{*}$, perturbation arguments give:

$$
\left\|\left(\tilde{P}(\mathbf{m})\left(H\left(\vec{\varkappa}^{(2)}\left(\varphi_{0}\right)\right)-k^{2} I\right) \tilde{P}(\mathbf{m})\right)^{-1}\right\| \geq \frac{1}{4} k^{\xi}, \quad \xi \geq r_{2}^{\prime}-1 .
$$

We will apply Lemma 5.14 to $\tilde{\Pi}(\mathbf{m})$ with $\xi=r_{2}^{\prime}-1$ in order to prove the lemma in hand in the same way we proved Lemma 4.18, using Lemmas 4.8, 4.10, 4.12, 4.13, 4.16, There are some technical complications though. Here is a detailed proof.

We start with considering simple boxes $\tilde{\Pi}(\mathbf{m}), \mathbf{m} \in \mathcal{M}^{(3)} \cap \Omega_{s}^{(2)}\left(r_{3}\right)$. Each box has the $\||\cdot| \mid-$-size $2 k^{r_{1} / 2}$ and contains no other than $\mathbf{m}$ elements of $\mathcal{M}^{(2)}\left(\varphi_{0}, r_{3}\right) \cup \Omega_{s}^{(2)}\left(r_{3}\right)$. Indeed, $\vec{\varkappa}^{(2)}\left(\varphi_{0}\right)$ satisfies the conditions of Lemma 3.31. This means that the $k^{\delta}$-cluster around each q: $\left\|\left|\vec{p}_{\mathbf{q}} \|\right|<k^{r_{1}}\right.$ is non-resonant. Since $\vec{\varkappa}=\vec{\varkappa}^{(2)}\left(\varphi_{0}\right)+\vec{p}_{\mathbf{m}}$ is a small perturbation of $\vec{\varkappa}^{(2)}\left(\varphi_{0}\right)$, the $k^{\delta}$-cluster around each $\mathbf{m}+\mathbf{q}$ : $\left\|\left|\vec{p}_{\mathbf{q}} \|\right|<k^{r_{1}}\right.$ is non-resonant too. This means $\mathbf{m}+\mathbf{q} \notin \mathcal{M}^{(2)}$. Further, $\mathbf{m}+\mathbf{q} \notin \Omega_{s}^{(2)}\left(r_{3}\right)$ by $(\underline{30})$, since $\mathbf{m} \in \Omega_{s}^{(2)}\left(r_{3}\right)$ and $\left\|\left|\vec{p}_{\mathbf{q}} \|\right|<k^{r_{1}}\right.$. Thus, $\mathbf{m}+\mathbf{q} \notin \mathcal{M}^{(2)}\left(\varphi_{0}, r_{3}\right) \cup \Omega_{s}^{(2)}\left(r_{3}\right)$. Next, we apply Lemma 5.14 with $\vec{\varkappa}_{0}=\vec{\varkappa}^{(2)}\left(\varphi_{0}\right)+\vec{p}_{\mathbf{m}_{0}}, J_{0}=1, \xi=r_{2}^{\prime}-1$, to conclude that the number of simple boxes $\tilde{\Pi}(\mathbf{m}), \mathbf{m} \in \mathcal{M}^{(3)} \cap \Omega_{s}^{(2)}\left(r_{3}\right)$ does not exceed $k^{\frac{2}{3} \gamma^{\prime} r_{2}+43 \gamma r_{1}}$. Indeed, we rewrite $\vec{\varkappa}^{(2)}\left(\varphi_{0}\right)+\vec{p}_{\mathbf{m}}$ in the form: $\vec{\varkappa}^{(2)}\left(\varphi_{0}\right)+\vec{p}_{\mathbf{m}}=\vec{\varkappa}_{0}+\vec{p}_{\mathbf{n}}, \mathbf{n}=\mathbf{m}-\mathbf{m}_{0} \in \Omega\left(\gamma^{\prime} r_{2}\right)$. By (327), $\vec{\varkappa}_{0}+\vec{p}_{\mathbf{n}} \in \mathcal{S}^{(2)}(k, \xi)$ (the operator in formula (327) having the size $2 k^{\frac{1}{2} r_{1}}$ and $\xi=r_{2}^{\prime}-1$, see (346)). Since, $\tilde{\Pi}(\mathbf{m})$ is simple, $\vec{\varkappa}_{0}+\vec{p}_{\mathbf{n}} \in \mathcal{N}\left(k, \gamma^{\prime} r_{2}, \vec{\varkappa}_{0}, 1\right)$ (here, $\gamma$ is taken to be equal to $1 / 2$ in the definition of $\left.\mathcal{S}^{(2)}(k, \xi)\right)$. Thus, $\vec{\varkappa}_{0}+\vec{p}_{\mathbf{n}} \in \mathcal{S}^{(2)}(k, \xi) \cap \mathcal{N}\left(k, \gamma^{\prime} r_{2}, \vec{\varkappa}_{0}, 1\right)$. By Lemma 5.14, the number of such $\vec{\varkappa}_{0}+\vec{p}_{\mathbf{n}}$ does not exceed $k^{2 \gamma^{\prime} r_{2} / 3+\frac{43}{2} r_{1}}$. Therefore, the number of $\mathcal{M}^{(3)}$ elements in simple boxes also does not exceed $k^{2 \gamma^{\prime} r_{2} / 3+\frac{43}{2} r_{1}}$.

Next, let us consider white clusters $\tilde{\Pi}(\mathbf{m})$, such that $\xi \geq k^{\frac{1}{6} \gamma r_{1}-2 \delta_{*}}$. Generally speaking, $\tilde{\Pi}(\mathbf{m})$ has a shape (in $\left.\mathbb{Z}^{4}\right)$ more complicated than a rectangular. However, each such cluster can be put in a box of the size $3 k^{\gamma r_{1} / 2+2 \delta_{0}}$, the box containing less than $k^{\frac{1}{6} \gamma r_{1}-\delta_{0} r_{1}}$ elements of $\mathcal{M}^{(2)}$ and the box resolvent satisfying (346) with $\xi=k^{\frac{1}{6} \gamma r_{1}-2 \delta_{*}}$ (Lemma 10.13). Applying Lemma 5.14 to such boxes $9\left(\vec{\varkappa}_{0}=\vec{\varkappa}^{(2)}\left(\varphi_{0}\right)+\vec{p}_{\mathbf{m}_{0}}, J_{0}=k^{\frac{1}{6} \gamma r_{1}-\delta_{0} r_{1}}, \xi=k^{\frac{1}{6} \gamma r_{1}-2 \delta_{*}}\right)$, we obtain that the number of $\Pi_{\mathbf{m}_{0}}$ points $\mathbf{m}$ in such boxes does not exceed $k^{2 \gamma^{\prime} r_{2} / 3+43 \gamma r_{1}}$. Similarly, we can treat grey boxes when $\xi \geq k^{\frac{1}{2} \gamma r_{1}+2 \delta_{0} r_{1}-2 \delta_{*}}$ (Lemma 10.14), black boxes when $\xi \geq k^{\gamma r_{1}+\delta_{0} r_{1}-2 \delta_{*}}$ (Lemma 10.15). However, in some cases $\xi$ does not satisfy the previous estimates from below. For such $\xi$ a somewhat more complicated construction is needed. Indeed, let us consider $\left(\tilde{P}\left(H\left(\vec{\varkappa}^{(2)}\left(\varphi_{0}\right)\right)-k^{2} I\right) \tilde{P}\right)^{-1}$ for $\tilde{\Pi}$ being white, grey or black cluster containing a point(s) of $\mathcal{M}^{(3)}$. A cluster $\tilde{\Pi}$ consists of blocks with the minimal size $k^{\gamma r_{1} / 6}$. Let us create a substructure inside $\tilde{\Pi}$. Namely, we construct white,

\footnotetext{
${ }^{9}$ Here we slightly abuse the notation of $J$ as Definition 5.10 deals with the estimates of the resolvent of a cluster while the points in $\mathcal{M}^{(2)}$ used in the definition of the colored boxes are defined by the distance to the poles of the cluster. Though the result of the form of Corollary 3.29 and simple perturbative arguments show that these two definitions are equivalent upto an insignificant factor.
} 
grey and black clusters corresponding to a smaller $\gamma_{\sim}$ which we denote by $\tilde{\gamma}, \tilde{\gamma}=10^{-4} \gamma$. Note, that there are no simple small clusters inside $\tilde{\Pi}$, since $\tilde{\Pi}$ is not simple. The size of these new clusters is much smaller than $k^{\gamma r_{1} / 6}$. However, they have properties analogous to those of the bigger clusters $(\gamma)$. These new clusters we call subclusters. We assert that at least one subcluster satisfies one of the following estimates (depending on whether this subcluster is white, grey or black):

$$
\begin{gathered}
\left\|\left(P_{w, s u b}\left(H\left(\vec{\varkappa}^{(2)}\left(\varphi_{0}\right)\right)-k^{2} I\right) P_{w, s u b}\right)^{-1}\right\|>k^{k^{\frac{\tilde{\gamma} r_{1}}{6}-2 \delta_{*}}}, \\
\left\|\left(P_{g, s u b}\left(H\left(\vec{\varkappa}^{(2)}\left(\varphi_{0}\right)\right)-k^{2} I\right) P_{g, s u b}\right)^{-1}\right\|>k^{k^{\left(\frac{\tilde{\gamma}}{2}+2 \tilde{\delta}_{0}\right) r_{1}-2 \delta_{*}}}, \\
\left\|\left(P_{b, s u b}\left(H\left(\vec{\varkappa}^{(2)}\left(\varphi_{0}\right)\right)-k^{2} I\right) P_{b, s u b}\right)^{-1}\right\|>k^{k^{\left(\tilde{\gamma}+\tilde{\delta}_{0}\right) r_{1}-2 \delta_{*}}},
\end{gathered}
$$

where $\tilde{\delta}_{0}=\tilde{\gamma} / 100$ (cf. definition of $\delta_{0}$ ). Indeed, if all subclusters satisfy the inequalities opposite to the inequalities above, then the perturbation series for the resolvent of the bigger cluster $(\gamma)$ (with respect to the block operator consisting of subclusters) converges, see the proof of Theorem 5.1, in particular the proof of (297) - (300). Hence, we have

$$
\left\|\left(\tilde{P}\left(H\left(\vec{\varkappa}^{(2)}\left(\varphi_{0}\right)\right)-k^{2} I\right) \tilde{P}\right)^{-1}\right\| \leq k^{k\left(\tilde{\gamma}+\tilde{\delta}_{0}\right) r_{1}-2 \delta_{*}},
$$

which contradicts to (346) under the hypothesis of the lemma $r_{2}^{\prime}>2 k^{\left(\gamma+\delta_{0}\right) 10^{-4} r_{1}-2 \delta_{*}}$.

Now, let us consider a resonant $k^{\gamma r_{1}}$-cluster $\tilde{\Pi}$, see (346), and the substructure inside. Note that each subcluster satisfying (347)-(349) can be treated the same way we treated the bigger clusters for large $\xi$. Namely, let us consider all $k^{\gamma r_{1}}$-clusters $\tilde{\Pi}$ for which there exists a white subcluster satisfying (347). By Lemma 10.14 each such subcluster can be put in a box of the size $3 k^{\tilde{\gamma} r_{1} / 2+2 \tilde{\delta}_{0}}$, the box resolvent satisfying (347). Such box has less than $k^{\left(\frac{\tilde{\gamma}}{6}-\tilde{\delta}_{0}\right) r_{1}}$ points $\mathbf{m}$ of $\mathcal{M}^{(2)}$. Now, applying Lemma 5.14 with $\vec{\varkappa}_{0}=\vec{\varkappa}^{(2)}\left(\varphi_{0}\right)+\vec{p}_{\mathbf{m}_{0}}$, $J_{0}=k^{\left(\frac{\tilde{\gamma}}{6}-\tilde{\delta}_{0}\right) r_{1}}, \xi=k^{\frac{\tilde{\gamma} r_{1}}{6}-2 \delta_{*}}$, we obtain that the number of points $\mathbf{m}$ in white subclusters (347) does not exceed $k^{\frac{2 \gamma^{\prime} r_{2}}{3}+43 \tilde{\gamma} r_{1}}$. Here we notice that condition of Lemma 5.14 holds, since $r_{2}<k^{\tilde{\delta}_{0} r_{1}-8 \delta_{*}}$ by (277). It follows that the number of $k^{\gamma r_{1}}$-clusters $\tilde{\Pi}(\mathbf{m})$, containing at least one white subcluster (347), does not exceed $k^{\frac{2 \gamma^{\prime} r_{2}}{3}+43 \tilde{\gamma} r_{1}}$.

Next, we consider all $k^{\gamma r_{1}}$-clusters $\tilde{\Pi}(\mathbf{m})$ for which there exists a grey subcluster, satisfying (348), but no white subclusters satisfying (347). Applying Lemma 10.14 and Lemma 5.14 with $J_{0}=k^{\left(\frac{\tilde{\gamma}}{2}+\tilde{\delta}_{0}\right) r_{1}}$ and $\xi=k^{\left(\frac{\tilde{\gamma}}{2}+2 \tilde{\delta}_{0}\right) r_{1}-2 \delta_{*}}$, we obtain that the number of such $k^{\gamma r_{1}}$-clusters $\tilde{\Pi}(\mathbf{m})$ in $\Pi_{\mathbf{m}_{0}}$ does not exceed $k^{\frac{2 \gamma^{\prime} r_{2}}{3}+43 \gamma r_{1}}$.

Similarly, applying Lemma 10.15 and Lemma 5.14 with $\xi=k^{\left(\tilde{\gamma}+\tilde{\delta}_{0}\right) r_{1}-2 \delta_{*}}$ and $J_{0}=$ $c k^{\tilde{\gamma} r_{1}+3}$, we obtain that the number of $k^{\gamma r_{1}}$-clusters $\tilde{\Pi}(\mathbf{m})$, containing a black subcluster (349) (and no grey or white subclusters, satisfying (347), (348)), does not exceed $k^{\frac{2 \gamma^{\prime} r_{2}}{3}+43 \gamma r_{1}}$. Here, we also used $r_{2}<k^{\tilde{\delta}_{0} r_{1}-3-8 \delta_{*}}$. 
Combining these estimates, we see that the number of clusters $\tilde{\Pi}$, containing at least one point of $\mathcal{M}^{(3)}$ does not exceed $k^{\frac{2 \gamma^{\prime} r_{2}}{3}+43 \gamma r_{1}}$. Taking into account that each $k^{\gamma r_{1}}$-cluster has a size not greater than $k^{\frac{3 \gamma r_{1}}{2}+3}$ and, hence, contains less than $k^{6 \gamma r_{1}+12}$ elements, we obtain that the total number of elements of $\mathcal{M}^{(3)}$ in $\Pi_{\mathbf{m}_{0}}$, does not exceed $k^{\frac{2 \gamma^{\prime} r_{2}}{3}+50 \gamma r_{1}}$.

We continue with constructing $k^{\gamma r_{1}}$-clusters in $\Omega\left(r_{3}\right), r_{3}>r_{2}$, the same way we did it for $\Omega\left(r_{2}\right)$ in Section 4.4.1. We call a $k^{\gamma r_{1}}$-cluster resonant if it contains $\mathbf{m} \in \mathcal{M}^{(3)}$, see (344). Next, we repeat the construction after Lemma 4.18, More precisely, let us split $\Omega\left(r_{3}\right) \backslash \Omega\left(r_{2}\right)$ into $k^{\gamma r_{2}}$-boxes, $\gamma=\frac{1}{5}$.

1. Simple region. Let $\Omega_{s}^{(3)}\left(r_{3}\right) \subset \Omega_{s}^{(2)}\left(r_{3}\right)$ be defined by the formula:

$$
\Omega_{s}^{(3)}\left(r_{3}\right)=\left\{\mathbf{m} \in \Omega\left(r_{3}\right), 0<p_{\mathbf{m}} \leq k^{-r_{2}^{\prime} k^{2 \gamma r_{1}}}\right\} .
$$

It is easy to see that $\Omega_{s}^{(3)}\left(r_{3}\right) \subset \mathcal{M}\left(\varphi_{0}, r_{3}\right)$, since $p_{\mathbf{m}}$ is small, see (74), (72)). Next, if $\mathbf{m} \in \Omega_{s}^{(3)}\left(r_{3}\right)$, then there are no other elements of $\mathcal{N}\left(\varphi_{0}, r_{3}\right)$ in the $k^{\delta}$-box around $\mathbf{m}$. Further, $\mathbf{m}$ itself can belong or do not belong to $\mathcal{M}^{(2)}\left(\varphi_{0}, r_{3}\right)$, but there are no other elements of $\mathcal{M}^{(2)}\left(\varphi_{0}, r_{3}\right)$ in the $k^{r_{1}}$-box around such $\mathbf{m}$. The proof of these facts is analogous to that in Step III, see "Simple region", page 67. Next, if $\mathbf{m} \in \Omega_{s}^{(3)}\left(r_{3}\right)$, then there are no other elements of $\Omega_{s}^{(3)}\left(r_{3}\right)$ in the surrounding $\||\cdot \||$-box of the size $k^{r_{2}}$, see (30). Last, $\mathbf{m}$ can belong or do not belong to $\mathcal{M}^{(3)}\left(\varphi_{0}, r_{3}\right)$, but there are no other elements from $\mathcal{M}^{(3)}\left(\varphi_{0}, r_{3}\right)$ in the $k^{r_{2}}$-box around such $\mathbf{m}$. Indeed, $\vec{\varkappa}^{(2)}\left(\varphi_{0}\right)$ satisfies the conditions of Lemma 4.28. This means that the $k^{\gamma r_{1}}$-cluster around each q: $0<\left\|\left|\vec{p}_{\mathbf{q}} \|\right|<k^{r_{2}}\right.$ is non-resonant. Since $\vec{\varkappa}=\vec{\varkappa}^{(2)}\left(\varphi_{0}\right)+\vec{p}_{\mathbf{m}}$ is a small perturbation of $\vec{\varkappa}^{(2)}\left(\varphi_{0}\right)$, the $k^{\gamma r_{1}}$-box around each $\mathbf{m}+\mathbf{q}: 0<\left\|\left|\vec{p}_{\mathbf{q}} \|\right|<k^{r_{2}}\right.$ is non-resonant too. This means $\mathbf{m}+\mathbf{q} \notin \mathcal{M}^{(3)}\left(\varphi_{0}, r_{3}\right)$.

For each $\mathbf{m} \in \Omega_{s}^{(3)}\left(r_{3}\right)$ we consider its $k^{r_{2} / 2}$-neighborhood. The union of such boxes we call the simple region and denote it by $\Pi_{s}\left(r_{3}\right)$. The corresponding projection is $P_{s}\left(r_{3}\right)$. Note that the distance from the simple region to the nearest point of $\mathcal{M}^{(3)}$ is greater than $\frac{1}{2} k^{r_{2}}$.

2. Black, grey and white regions are defined in the same way as in the construction after Lemma 4.18 with $r_{3}$ instead of $r_{2}, r_{2}$ instead of $r_{1}, \mathcal{M}^{(3)}$ instead of $\mathcal{M}^{(2)}$ and the restriction $p_{\mathbf{m}}>k^{-r_{2}^{\prime} k^{2 \gamma r_{1}}}$ instead of $p_{\mathbf{m}}>k^{-5 r_{1}^{\prime}}$. We continue to use notation $P_{b}, P_{g}, P_{g}^{\prime}, P_{w}, P_{w}^{\prime}$ and $\Pi_{b}, \Pi_{g}, \Pi_{g}^{\prime}, \Pi_{w}, \Pi_{w}^{\prime}$. Sometimes, where it can lead to confusion we will write $P_{b}\left(r_{3}\right)$ etc. to distinguish these objects from the ones introduced in Step II.

3. Non-resonant region. Now, the non-resonant region consists of two parts: the simpler part which was non-resonant already in the previous step and the part which is new for the current step. Namely, first we consider $k^{\delta}$-components corresponding 
to points in the set $\mathcal{M}\left(r_{3}, \varphi_{0}\right) \backslash\left(\mathcal{M}\left(r_{2}, \varphi_{0}\right) \cup \mathcal{M}^{(2)}\left(r_{3}, \varphi_{0}\right) \cup \Omega_{s}^{(2)}\left(r_{3}\right) \cup \mathcal{M}_{2, t w}\left(r_{3}, \varphi_{0}\right)\right)$. The union of this neighborhoods we denote $\Pi_{n r, \delta}$. The corresponding projection is $P_{n r, \delta}$. These $k^{\delta}$-clusters can be treated by means of the second step. We also consider all points in the set $\mathcal{M}^{(2)}\left(r_{3}, \varphi_{0}\right) \cup \Omega_{s}^{(2)}\left(r_{3}\right) \backslash\left(\mathcal{M}^{(2)}\left(r_{2}, \varphi_{0}\right) \cup \mathcal{M}^{(3)}\left(r_{3}, \varphi_{0}\right) \cup \Omega_{s}^{(3)}\left(r_{3}\right)\right)$. We construct simple, white, grey and black clusters around them exactly as in preparation to Step III. The union of these clusters we denote $\Pi_{n r, r_{1}}$. The corresponding projection is $P_{n r, r_{1}}$. The set $\Pi_{n r}:=\Pi_{n r, \delta} \cup \Pi_{n r, r_{1}}$ is called the non-resonant set with $P_{n r}$ being the corresponding projection. The part of the non-resonant region which is outside $\Pi_{s} \cup \Pi_{b} \cup \Pi_{g} \cup \Pi_{w}$, we denote $\Pi_{n r}^{\prime}$ and the corresponding projection by $P_{n r}^{\prime}$.

We put as before

$$
P_{r}\left(r_{3}\right):=P_{s}\left(r_{3}\right)+P_{b}\left(r_{3}\right)+P_{g}^{\prime}\left(r_{3}\right)+P_{w}^{\prime}\left(r_{3}\right), \quad P^{(3)}:=P_{r}\left(r_{3}\right)+P_{n r}^{\prime}\left(r_{3}\right)+P\left(r_{2}\right) .
$$

We also continue to use the similar agreement in the notation which we set in Step II. We just note that now we use $r_{2}$ rather than $r_{1}$ to establish equivalence between the boxes.

We continue construction from Step II. Repeating the arguments from the proofs of Lemmas 4.19, 4.20, 4.21 with obvious changes (in particular, using Lemma 5.15 instead of Lemma 4.18) we obtain the following results.

Lemma 5.16. 1. Each $\Pi_{b}^{j}$ contains no more than $k^{\gamma r_{2} / 2-\delta_{0} r_{2}+150 \gamma r_{1}}$ black boxes.

2. The size of $\Pi_{b}^{j}$ in $\left\||\cdot \||\right.$ norm is less than $k^{3 \gamma r_{2} / 2+150 \gamma r_{1}}$.

3. Each $\Pi_{b}^{j}$ contains no more than $k^{\gamma r_{2}+150 \gamma r_{1}}$ elements of $\mathcal{M}^{(3)}$. Moreover, any box of $\||\cdot| \mid-$-size $k^{3 \gamma r_{2} / 2+150 \gamma r_{1}}$ containing $\Pi_{b}^{j}$ has no more than $k^{\gamma r_{2}+150 \gamma r_{1}}$ elements of $\mathcal{M}^{(3)}$ inside.

Lemma 5.17. 1. Each $\Pi_{g}^{j}$ contains no more than $k^{\gamma r_{2} / 3+2 \delta_{0} r_{2}}$ grey boxes.

2. The size of $\Pi_{g}^{j}$ in $\left\||\cdot \||\right.$ norm is less than $k^{5 \gamma r_{2} / 6+4 \delta_{0} r_{2}}$.

3. Each $\Pi_{g}^{j}$ contains no more than $k^{\gamma r_{2} / 2+\delta_{0} r_{2}}$ elements of $\mathcal{M}^{(3)}$.

Lemma 5.18. 1. The size of $\Pi_{w}^{j}$ in $\left\||\cdot \||\right.$ norm is less than $k^{\gamma r_{2} / 3-\delta_{0} r_{2}}$.

2. Each $\Pi_{w}^{j}$ contains no more than $k^{\gamma r_{2} / 6-\delta_{0} r_{2}}$ points of $\mathcal{M}^{(3)}$.

The construction of the rest of Section 4.4.1 stays unchanged. Let us introduce corresponding notation, formulate the results and provide some comments.

Next lemmas are the analogues of Lemmas 4.24, 4.25, 4.26. 
Lemma 5.19. Let $\varphi_{0} \in \omega^{(3)}(k, \delta, \tau),\left|\varphi-\varphi_{0}\right|<k^{-k^{r} 1}$. Then,

$$
\left\|\left(P_{n r}\left(H\left(\vec{\varkappa}^{(3)}(\varphi)\right)-k^{2} I\right) P_{n r}\right)^{-1}\right\|<k^{r_{2}^{\prime} k^{2 \gamma r_{1}}} k^{r_{2}^{\prime}} \leq k^{k^{3 \gamma r_{1}}} .
$$

Proof. Construction in Section 4.4 is made for an arbitrary large $r_{2}$. Here we repeat it for $r_{3}$. We use Lemma 4.24 for $\Pi_{n r, \delta}$, Lemma 4.25 for white, grey and black clusters $\left(\varepsilon_{0}=k^{-r_{2}^{\prime}}\right)$. We also use Lemma 4.26 $\left(p_{\mathbf{m}}>k^{-r_{2}^{\prime} k^{2 \gamma r_{1}}}, \varepsilon_{0}=k^{-r_{2}^{\prime}}\right)$, for simple clusters in $\Pi_{n r, r_{1}}$. We also use (325). All together the estimates for the clusters resolvents yield (352). The estimate (352) is stable when $\left|\varphi-\varphi_{0}\right|<k^{-k^{r_{1}}}$, since $k^{-k^{r_{1}+2}}=o\left(k^{-k^{3 \gamma r_{1}}}\right)$.

Lemma 5.20. Let $\varphi_{0} \in \omega^{(3)}(k, \delta, \tau)$, and $\left|\varphi-\varphi_{0}\right|<k^{-k^{r_{1}}}, i=1,2,3$. Then,

1. The number of poles of the resolvent $\left(P_{i}\left(H\left(\vec{\varkappa}^{(3)}(\varphi)\right)-k^{2} I\right) P_{i}\right)^{-1}$ in the disc $\mid \varphi-$ $\varphi_{0} \mid<k^{-k^{r_{1}}}$ is no greater than $N_{i}^{(2)}$, where $N_{1}^{(2)}=k^{\gamma r_{2}+150 \gamma r_{1}}, N_{2}^{(2)}=k^{\gamma r_{2} / 2+\delta_{0} r_{2}}$, $N_{3}^{(2)}=k^{\gamma r_{2} / 6-\delta_{0} r_{2}}$.

2. Let $\varepsilon$ be the distance to the nearest pole of the resolvent in $\mathcal{W}^{(3)}$ and $\varepsilon_{0}=\min \left\{\varepsilon, k^{-r_{2}^{\prime}}\right\}$. Then the following estimates hold:

$$
\begin{array}{r}
\left\|\left(P_{i}\left(H\left(\vec{\varkappa}^{(3)}(\varphi)\right)-k^{2} I\right) P_{i}\right)^{-1}\right\|<k^{2 r_{2}^{\prime} k^{2 \gamma r_{1}}} k^{r_{2}^{\prime}}\left(\frac{k^{-r_{2}^{\prime}}}{\varepsilon_{0}}\right)^{N_{i}^{(2)}} \leq \\
k^{k^{3 \gamma r_{1}}}\left(\frac{k^{-r_{2}^{\prime}}}{\varepsilon_{0}}\right)^{N_{i}^{(2)}}, \\
\left\|\left(P_{i}\left(H\left(\vec{\varkappa}^{(3)}(\varphi)\right)-k^{2} I\right) P_{i}\right)^{-1}\right\|_{1}<k^{2 r_{2}^{\prime} k^{2 \gamma r_{1}}} k^{r_{2}^{\prime}+8 \gamma r_{2}}\left(\frac{k^{-r_{2}^{\prime}}}{\varepsilon_{0}}\right)^{N_{i}^{(2)}} \leq \\
k^{k^{3 \gamma r_{1}}}\left(\frac{k^{-r_{2}^{\prime}}}{\varepsilon_{0}}\right)^{N_{i}^{(2)}} .
\end{array}
$$

Proof. The proof of this lemma is analogous to that of Lemma $4.25 \mathrm{up}$ to the replacement of $\mathcal{M}^{(2)}$ by $\mathcal{M}^{(3)}, \mathcal{O}_{\mathbf{m}}^{(2)}$ by $\mathcal{O}_{\mathbf{m}}^{(3)}$, and the shift of indices: $\delta$ to $r_{1}, r_{1}$ to $r_{2}$, etc. We apply Lemmas 5.16 5.18 instead of 4.19 4.21 and Lemmas 4.25, 4.26 with $\varepsilon_{0}=k^{-r_{2}^{\prime}}$ and $p_{\mathbf{m}}>k^{-r_{2}^{\prime} k^{2 \gamma r_{1}}}$ instead of Lemma 3.22. We also note that $N_{i}^{(1)}<k^{2 \gamma r_{1}}$ in (264), (265).

Lemma 5.21. Let $\varphi_{0} \in \omega^{(3)}(k, \delta, \tau)$. Then, the operator $\left(P_{s}^{j}\left(H\left(\vec{\varkappa}^{(3)}(\varphi)\right)-k^{2} I\right) P_{s}^{j}\right)^{-1}$ has no more than one pole in the disk $\left|\varphi-\varphi_{0}\right|<k^{-k^{r_{1}}}$. Moreover,

$$
\left\|\left(P_{s}^{j}\left(H\left(\vec{\varkappa}^{(3)}(\varphi)\right)-k^{2} I\right) P_{s}^{j}\right)^{-1}\right\|<\frac{8 k^{-1}}{p_{\mathbf{m}} \varepsilon_{0}}
$$




$$
\left\|\left(P_{s}^{j}\left(H\left(\vec{\varkappa}^{(3)}(\varphi)\right)-k^{2} I\right) P_{s}^{j}\right)^{-1}\right\|_{1}<\frac{8 k^{-1+4 r_{2}}}{p_{\mathbf{m}} \varepsilon_{0}},
$$

$\varepsilon_{0}=\min \left\{\varepsilon, k^{-r_{2}^{\prime}}\right\}$, where $\varepsilon$ is the distance to the pole of the operator.

Note that $p_{\mathbf{m}}>k^{-\mu r_{3}}$ when $\mathbf{m} \in \Omega\left(r_{3}\right)$. The analogues of Lemma 4.22 and Corollary 4.23 also hold.

\subsubsection{Resonant and Nonresonant Sets for Step IV}

We divide $[0,2 \pi)$ into $\left[2 \pi k^{k^{r_{1}}}\right]+1$ intervals $\Delta_{l}^{(3)}$ with the length not bigger than $k^{-k^{r_{1}}}$. If a particular interval belongs to $\mathcal{O}^{(3)}$ we ignore it; otherwise, let $\varphi_{0}^{(l)} \notin \mathcal{O}^{(3)}$ be a point inside the $\Delta_{l}^{(3)}$. Let

$$
\mathcal{W}_{l}^{(3)}=\left\{\varphi \in \mathcal{W}^{(3)}:\left|\varphi-\varphi_{0}^{(l)}\right|<4 k^{-k^{r} 1}\right\}
$$

Clearly, neighboring sets $\mathcal{W}_{l}^{(3)}$ overlap (because of the multiplier 4 in the inequality), they cover $\hat{\mathcal{W}}^{(3)}$, which is the restriction of $\mathcal{W}^{(3)}$ to the $2 k^{-k^{r_{1}}}$-neighborhood of $[0,2 \pi)$. For each $\varphi \in \hat{\mathcal{W}}^{(3)}$ there is an $l$ such that $\left|\varphi-\varphi_{0}^{(l)}\right|<4 k^{-k^{r_{1}}}$. We consider the poles of the resolvent $\left(P^{(3)}\left(H\left(\overrightarrow{\mathcal{\varkappa}}^{(3)}(\varphi)\right)-k^{2}\right) P^{(3)}\right)^{-1}$ in $\hat{\mathcal{W}}_{l}^{(3)}$ and denote them by $\varphi_{l m}^{(3)}, m=1, \ldots, M_{m}$. As before, the resolvent has a block structure. The number of blocks clearly cannot exceed the number of elements in $\Omega\left(r_{3}\right)$, i.e. $k^{4 r_{3}}$. Using the estimates for the number of poles for each block, the estimate being provided by Lemma 5.20, Part 1, we can roughly estimate the number of poles of the resolvent by $k^{4 r_{3}+r_{2}}$. Next, let $r_{3}^{\prime}>k^{r_{1}}$ and $\mathcal{O}_{l m}^{(4)}$ be the disc of the radius $k^{-r_{3}^{\prime}}$ around $\varphi_{l m}^{(3)}$.

Definition 5.22. The set

$$
\mathcal{O}^{(4)}=\cup_{l m} \mathcal{O}_{l m}^{(4)}
$$

we call the fourth resonant set. The set

$$
\mathcal{W}^{(4)}=\hat{\mathcal{W}}^{(3)} \backslash \mathcal{O}^{(4)}
$$

is called the fourth non-resonant set. The set

$$
\omega^{(4)}=\mathcal{W}^{(4)} \cap[0,2 \pi)
$$

is called the fourth real non-resonant set.

The following statements can be proven in the same way as Lemmas 4.28, 4.29] and 4.30 . 
Lemma 5.23. Let $r_{3}^{\prime}>\mu r_{3}>k^{r_{1}}, \varphi \in \mathcal{W}^{(4)}, \varphi_{0}^{(l)}$ corresponds to an interval $\Delta_{l}^{(3)}$ containing $\Re \varphi$. Let $\Pi$ be one of the components $\Pi_{s}^{j}\left(\varphi_{0}^{(l)}\right), \Pi_{b}^{j}\left(\varphi_{0}^{(l)}\right), \Pi_{g}^{j}\left(\varphi_{0}^{(l)}\right), \Pi_{w}^{j}\left(\varphi_{0}^{(l)}\right)$ and $P(\Pi)$ be the projection corresponding to $\Pi$. Let also $\varkappa \in \mathbb{C}:\left|\varkappa-\varkappa^{(3)}(\varphi)\right|<k^{-r_{3}^{\prime} k^{2 \gamma r_{2}}}$. Then,

$$
\begin{gathered}
\left\|\left(P(\Pi)\left(H(\vec{\varkappa}(\varphi))-k^{2} I\right) P(\Pi)\right)^{-1}\right\|<k^{2 \mu r_{3}+r_{3}^{\prime} N^{(2)}}, \\
\left\|\left(P(\Pi)\left(H(\vec{\varkappa}(\varphi))-k^{2} I\right) P(\Pi)\right)^{-1}\right\|_{1}<k^{(2 \mu+1) r_{3}+r_{3}^{\prime} N^{(2)}},
\end{gathered}
$$

$N^{(2)}$ corresponding to the color of $\Pi\left(N^{(2)}=1, k^{\gamma r_{2}+150 \gamma r_{1}}, k^{\gamma r_{2} / 2+\delta_{0} r_{2}}, k^{\gamma r_{2} / 6-\delta_{0} r_{2}}\right.$ for simple, black, grey and white clusters, correspondingly).

By total size of the set $\mathcal{O}^{(4)}$ we mean the sum of the sizes of its connected components.

Lemma 5.24. Let $r_{3}^{\prime} \geq(\mu+10) r_{3}, r_{3}>k^{r_{1}}$. Then, the size of each connected component of $\mathcal{O}^{(4)}$ is less than $32 k^{4 r_{3}-r_{3}^{\prime}}$. The total size of $\mathcal{O}^{(4)}$ is less than $k^{-r_{3}^{\prime} / 2}$.

Lemma 5.25. Let $\varphi \in \mathcal{W}^{(3)}$ and $C_{4}$ be the circle $\left|z-k^{2}\right|=k^{-2 r_{3}^{\prime} k^{2 \gamma r_{2}}}$. Then

$$
\left\|\left(P\left(r_{2}\right)\left(H\left(\vec{\varkappa}^{(3)}(\varphi)\right)-z\right) P\left(r_{2}\right)\right)^{-1}\right\| \leq 4^{3} k^{2 r_{3}^{\prime} k^{2 \gamma r_{2}}} .
$$

We prove this lemma using (293).

\section{STEP IV}

\subsection{Operator $H^{(4)}$. Perturbation Formulas}

Let $P\left(r_{3}\right)$ be an orthogonal projector onto $\Omega\left(r_{3}\right):=\left\{\mathbf{m}:\left\|\vec{p}_{\mathbf{m}}\right\| \mid \leq k^{r_{3}}\right\}$ and $H^{(4)}=$ $P\left(r_{3}\right) H P\left(r_{3}\right)$. From now on, we assume

$$
k^{r_{1}}<r_{3}<k^{\gamma 10^{-7} r_{2}}, \quad k^{2 \gamma 10^{-4} r_{2}}<r_{3}^{\prime}<k^{\delta_{0} r_{2} / 2} .
$$

We consider $H^{(4)}\left(\vec{\varkappa}^{(3)}(\varphi)\right)$ as a perturbation of $\tilde{H}^{(3)}\left(\vec{\varkappa}^{(3)}(\varphi)\right)$ :

$$
\tilde{H}^{(3)}:=\tilde{P}_{l}^{(3)} H \tilde{P}_{l}^{(3)}+\left(P\left(r_{3}\right)-\tilde{P}_{l}^{(3)}\right) H_{0},
$$

where $H=H\left(\vec{\varkappa}^{(3)}(\varphi)\right), H_{0}=H_{0}\left(\vec{\varkappa}^{(3)}(\varphi)\right)$ and $\tilde{P}_{l}^{(3)}$ is the projection $P^{(3)}$ corresponding to $\varphi_{0}^{(l)}$ in the interval $\Delta_{l}^{(3)}$ containing $\varphi$, see (351). Note that the operator $\tilde{H}^{(3)}$ has a block structure, the block $\tilde{P}_{l}^{(3)} H \tilde{P}_{l}^{(3)}$ being composed of smaller blocks $P_{i} H P_{i}, i=0, \ldots, 5$. By analogy with (279)-(281),

$$
W^{(3)}=H^{(4)}-\tilde{H}^{(3)}=P\left(r_{3}\right) V P\left(r_{3}\right)-\tilde{P}_{l}^{(3)} V \tilde{P}_{l}^{(3)},
$$




$$
\begin{gathered}
g_{r}^{(4)}(\vec{\varkappa}):=\frac{(-1)^{r}}{2 \pi i r} \operatorname{Tr} \oint_{C_{4}}\left(W^{(3)}\left(\tilde{H}^{(3)}(\vec{\varkappa})-z I\right)^{-1}\right)^{r} d z, \\
G_{r}^{(4)}(\vec{\varkappa}):=\frac{(-1)^{r+1}}{2 \pi i} \oint_{C_{4}}\left(\tilde{H}^{(3)}(\vec{\varkappa})-z I\right)^{-1}\left(W^{(3)}\left(\tilde{H}^{(3)}(\vec{\varkappa})-z I\right)^{-1}\right)^{r} d z,
\end{gathered}
$$

where $C_{4}$ is the circle $\left|z-k^{2}\right|=\varepsilon_{0}^{(4)}, \varepsilon_{0}^{(4)}=k^{-2 r_{3}^{\prime} k^{2 \gamma r_{2}}}$.

The proof of the following statements is analogous to the one in the previous step (see Theorem 5.1. Corollary 5.2 and Lemma 5.3) up to the replacement of $r_{3}$ by $r_{4}, r_{2}$ by $r_{3}$, $r_{1}$ by $r_{2}$, etc.

Theorem 6.1. Suppose $k>k_{*}, \varphi$ is in the real $k^{-r_{3}^{\prime}-\delta}$-neighborhood of $\omega^{(4)}(k, \delta, \tau)$ and $\varkappa \in \mathbb{R},\left|\varkappa-\varkappa^{(3)}(\varphi)\right| \leq \varepsilon_{0}^{(4)} k^{-1-\delta}, \vec{\varkappa}=\varkappa(\cos \varphi, \sin \varphi)$. Then, there exists a single eigenvalue of $H^{(4)}(\vec{\varkappa})$ in the interval $\varepsilon_{4}(k, \delta, \tau)=\left(k^{2}-\varepsilon_{0}^{(4)}, k^{2}+\varepsilon_{0}^{(4)}\right)$. It is given by the absolutely converging series:

$$
\lambda^{(4)}(\vec{\varkappa})=\lambda^{(3)}(\vec{\varkappa})+\sum_{r=2}^{\infty} g_{r}^{(4)}(\vec{\varkappa}) .
$$

For coefficients $g_{r}^{(4)}(\vec{\varkappa})$ the following estimates hold:

$$
\left|g_{r}^{(4)}(\vec{\varkappa})\right|<k^{-\frac{\beta}{5} k^{r_{2}-r_{1}}-\beta(r-1)} .
$$

The corresponding spectral projection is given by the series:

$$
\mathcal{E}^{(4)}(\vec{\varkappa})=\mathcal{E}^{(3)}(\vec{\varkappa})+\sum_{r=1}^{\infty} G_{r}^{(4)}(\vec{\varkappa}),
$$

$\mathcal{E}^{(3)}(\vec{\varkappa})$ being the spectral projection of $H^{(3)}(\vec{\varkappa})$. The operators $G_{r}^{(4)}(\vec{\varkappa})$ satisfy the estimates:

$$
\begin{gathered}
\left\|G_{r}^{(4)}(\vec{\varkappa})\right\|_{1}<k^{-\frac{\beta}{10} k^{r_{2}-r_{1}}-\beta r}, \\
G_{r}^{(4)}(\vec{\varkappa})_{\mathbf{s s}^{\prime}}=0 \text {, when } 2 r k^{\gamma r_{2}+150 \gamma r_{1}}+3 k^{r_{2}}<\left\|\left|\vec { p } _ { \mathbf { s } } \left\|\left|+\left\|\mid \vec{p}_{\mathbf{s}^{\prime}}\right\| \| .\right.\right.\right.\right.
\end{gathered}
$$

Corollary 6.2. For the perturbed eigenvalue and its spectral projection the following estimates hold:

$$
\begin{gathered}
\lambda^{(4)}(\vec{\varkappa})=\lambda^{(3)}(\vec{\varkappa})+O_{2}\left(k^{-\frac{1}{5} \beta k^{r_{2}-r_{1}}}\right), \\
\left\|\mathcal{E}^{(4)}(\vec{\varkappa})-\mathcal{E}^{(3)}(\vec{\varkappa})\right\|_{1}<k^{-\frac{\beta}{10} k^{r_{2}-r_{1}}} . \\
\left|\mathcal{E}^{(4)}(\vec{\varkappa})_{\mathbf{s s}^{\prime}}\right|<k^{-d^{(4)}\left(\mathbf{s}, \mathbf{s}^{\prime}\right)}, \quad \text { when }\left\|\left|\vec{p}_{\mathbf{s}} \|\right|>4 k^{r_{2}} \text { or }\right\|\left|\vec{p}_{\mathbf{s}^{\prime}} \|\right|>4 k^{r_{2}}, \\
d^{(4)}\left(\mathbf{s}, \mathbf{s}^{\prime}\right)=\frac{1}{16}\left(\left\|\left|\vec { p } _ { \mathbf { s } } \left\|\left|+\left\|\left|\vec{p}_{\mathbf{s}^{\prime}} \|\right|\right) k^{-\gamma r_{2}-150 \gamma r_{1}} \beta+\frac{1}{10} \beta k^{r_{2}-r_{1}} .\right.\right.\right.\right.\right.
\end{gathered}
$$


Lemma 6.3. Under conditions of Theorem 6.1 the following estimates hold when $\varphi \in$ $\omega^{(4)}(k, \delta)$ or its complex $k^{-r_{3}^{\prime}-\delta}$-neighborhood and $\varkappa \in \mathbb{C}:\left|\varkappa-\varkappa^{(3)}(\varphi)\right|<\varepsilon_{0}^{(4)} k^{-1-\delta}$.

$$
\begin{gathered}
\lambda^{(4)}(\vec{\varkappa})=\lambda^{(3)}(\vec{\varkappa})+O_{2}\left(k^{-\frac{1}{5} \beta k^{r_{2}-r_{1}}}\right) \\
\frac{\partial \lambda^{(4)}}{\partial \varkappa}=\frac{\partial \lambda^{(3)}}{\partial \varkappa}+O_{2}\left(k^{-\frac{1}{5} \beta k^{r_{2}-r_{1}}} M_{2}\right), \quad M_{2}:=\frac{k^{1+\delta}}{\varepsilon_{0}^{(4)}} \\
\frac{\partial \lambda^{(4)}}{\partial \varphi}=\frac{\partial \lambda^{(3)}}{\partial \varphi}+O_{2}\left(k^{-\frac{1}{5} \beta k^{r_{2}-r_{1}}+r_{3}^{\prime}+\delta}\right) \\
\frac{\partial^{2} \lambda^{(4)}}{\partial \varkappa^{2}}=\frac{\partial^{2} \lambda^{(3)}}{\partial \varkappa^{2}}+O_{2}\left(k^{-\frac{1}{5} \beta k^{r_{2}-r_{1}}} M_{2}^{2}\right) \\
\frac{\partial^{2} \lambda^{(4)}}{\partial \varkappa \partial \varphi}=\frac{\partial^{2} \lambda^{(3)}}{\partial \varkappa \partial \varphi}+O_{2}\left(k^{-\frac{1}{5} \beta k^{r_{2}-r_{1}}+r_{3}^{\prime}+\delta} M_{2}\right) \\
\frac{\partial^{2} \lambda^{(4)}}{\partial \varphi^{2}}=\frac{\partial^{2} \lambda^{(3)}}{\partial \varphi^{2}}+O_{2}\left(k^{-\frac{1}{5} \beta k^{r_{2}-r_{1}}+2 r_{3}^{\prime}+2 \delta}\right) .
\end{gathered}
$$

Corollary 6.4. All " $\mathrm{O}_{2}$ "-s on the right hand sides of (375)-(380) can be written as $O_{1}\left(k^{-\frac{1}{10} \beta k^{r_{2}-r_{1}}}\right)$.

Remark 6.5. In the proof of Theorem 6.1 and similar statements in every further step of the induction we obtain the estimate of the form (292). It is important to notice that the right hand side of these estimates is always $k^{-2 \beta}$. It can't become better since it comes, in particular, from the estimate of the free resolvent on the set of points satisfying ||$\vec{\varkappa}+\left.\vec{p}_{\mathbf{m}}\right|_{\mathbb{R}} ^{2}-k^{2} \mid \geq k^{\delta_{*}}$. What changes is the first term in the perturbation series, see e.g. (283), (285) vs (368), (370).

\subsection{Isoenergetic Surface for Operator $H^{(4)}$}

The following statement is an analogue of Lemma 5.5.

Lemma 6.6. 1. For every $\lambda:=k^{2}, k>k_{*}$, and $\varphi$ in the real $\frac{1}{2} k^{-r_{3}^{\prime}-\delta}$-neighborhood of $\omega^{(4)}(k, \delta, \tau)$, there is a unique $\varkappa^{(4)}(\lambda, \varphi)$ in the interval $I_{3}:=\left[\varkappa^{(3)}(\lambda, \varphi)-\right.$ $\left.\varepsilon_{0}^{(4)} k^{-1-\delta}, \varkappa^{(3)}(\lambda, \varphi)+\varepsilon_{0}^{(4)} k^{-1-\delta}\right]$, such that

$$
\lambda^{(4)}\left(\vec{\varkappa}^{(4)}(\lambda, \varphi)\right)=\lambda, \quad \vec{\varkappa}^{(4)}(\lambda, \varphi):=\varkappa^{(4)}(\lambda, \varphi) \vec{\nu}(\varphi) .
$$

2. Furthermore, there exists an analytic in $\varphi$ continuation of $\varkappa^{(4)}(\lambda, \varphi)$ to the complex $\frac{1}{2} k^{-r_{3}^{\prime}-\delta}$-neighborhood of $\omega^{(4)}(k, \delta, \tau)$ such that $\lambda^{(4)}\left(\vec{\varkappa}^{(4)}(\lambda, \varphi)\right)=\lambda$. Function $\varkappa^{(4)}(\lambda, \varphi)$ can be represented as $\varkappa^{(4)}(\lambda, \varphi)=\varkappa^{(3)}(\lambda, \varphi)+h^{(4)}(\lambda, \varphi)$, where

$$
\left|h^{(4)}(\varphi)\right|=O_{1}\left(k^{-\frac{1}{5} \beta k^{r_{2}-r_{1}}-1}\right) \text {, }
$$




$$
\frac{\partial h^{(4)}}{\partial \varphi}=O_{2}\left(k^{-\frac{1}{5} \beta k^{r_{2}-r_{1}-1+r_{3}^{\prime}+\delta}}\right), \quad \frac{\partial^{2} h^{(4)}}{\partial \varphi^{2}}=O_{4}\left(k^{-\frac{1}{5} \beta k^{r_{2}-r_{1}}-1+2 r_{3}^{\prime}+2 \delta}\right) .
$$

Let us consider the set of points in $\mathbb{R}^{2}$ given by the formula: $\vec{\varkappa}=\vec{\varkappa}^{(4)}(\varphi), \quad \varphi \in$ $\omega^{(4)}(k, \delta, \tau)$. By Lemma 6.6 this set of points is a slight distortion of $\mathcal{D}_{3}$. All the points of this curve satisfy the equation $\lambda^{(4)}\left(\vec{\varkappa}^{(4)}(\varphi)\right)=k^{2}$. We call it isoenergetic surface of the operator $H^{(4)}$ and denote by $\mathcal{D}_{4}$.

\section{Induction}

\subsection{Inductive formulas for $r_{n}$}

Now, we are ready to introduce the induction. In fact, STEP IV has been the first inductive step. Here, for the sake of convenience, we reformulate the main statements from the previous step in terms of $r_{n}, n \geq 3$, and provide necessary comments. First, we choose

$$
k^{r_{n-2}}<r_{n}<k^{\gamma 10^{-7} r_{n-1}}, \quad k^{2 \gamma 10^{-4} r_{n-1}}<r_{n}^{\prime}<k^{\delta_{0} r_{n-1} / 2}, \quad n \geq 3 .
$$

\subsection{Preparation for Step $n+1, n \geq 4$}

\subsubsection{Properties of the Quasiperiodic Lattice. Induction}

Here we prove the inductive version of the results from Section 5.3.1. We consider $\vec{p}_{\mathbf{m}}=$ $2 \pi\left(\mathbf{s}_{1}+\alpha \mathbf{s}_{2}\right)$ with integer vectors $\mathbf{s}_{j}$ such that $\left|\mathbf{s}_{j}\right| \leq 4 k^{r_{n-1}}$. We repeat the arguments from the beginning of Section 4.3. Namely, let $(q, p) \in \mathbb{Z}^{2}$ be a pair such that $0<q \leq 4 k^{r_{n-1}}$ and

$$
|\alpha q+p| \leq \frac{1}{4} k^{-r_{n-1}}
$$

We choose a pair $(p, q)$ which gives the best approximation. In particular, $p$ and $q$ are mutually prime. Put $\epsilon_{q}:=\alpha+\frac{p}{q}$. We have

$$
k^{-2 r_{n-1} \mu} \leq\left|\epsilon_{q}\right| \leq \frac{1}{4} q^{-1} k^{-r_{n-1}} .
$$

The analogs of Lemmas $5 . 6 \longdiv { 5 . 8 }$ hold with $n-1$ instead of 2 .

We consider the matrix $H^{(n-1)}(\vec{\varkappa})=P\left(\gamma r_{n-2}\right) H(\vec{\varkappa})\left(\gamma r_{n-2}\right)$ where $\vec{\varkappa} \in \mathbb{R}^{2}, P\left(\gamma r_{n-2}\right)$ is the orthogonal projection corresponding to $\Omega\left(\gamma r_{n-2}\right)$ (it is a slight abuse of notations, since $H^{(n-1)}$ in Step $n-1$ was defined for $\gamma=1$ ). We construct the block structure in $H^{(n-1)}(\vec{\varkappa})$ analogous to that in Step $n-1$. The difference is that now we consider any $\vec{\varkappa} \in \mathbb{R}^{2}$, not only $\vec{\varkappa}$ being close to $\vec{\varkappa}^{(n-2)}(\varphi)$. Correspondingly, we define non-resonant $\mathbf{m}$ not in terms of $\varphi$, but in more general terms of inequalities providing convergence of perturbation series. Indeed, we call $\mathbf{m} \in \Omega\left(\gamma r_{n-2}\right)$ non-resonant if (cf. (72))

$$
|| \vec{\varkappa}+\left.\vec{p}_{\mathbf{m}}\right|^{2}-k^{2} \mid>k^{\delta_{*}} \text {. }
$$


Obviously, this estimate is stable in the $k^{-\delta_{*}-1-\delta}$-neighborhood of a given $\vec{\varkappa}$. Hence, the definition of a non-resonant $\mathbf{m}$ is stable in this neighborhood up to a multiplier $1+o(1)$ in the r.h.s. of (387). Around each resonant $\mathbf{m}$ (which is also not trivial weakly resonant in the sense of Step II) we construct $k^{\delta}$-boxes/clusters (see (145)). Let $P(\mathbf{m})$ be the projection on the $k^{\delta}$-cluster containing $\mathbf{m}$. If

$$
\left\|\left(P(\mathbf{m})\left(H(\vec{\varkappa})-k^{2}\right) P(\mathbf{m})\right)^{-1}\right\|<k^{4 \gamma r_{1}^{\prime}}
$$

(cf. Definition [5.9), then we call the $k^{\delta}$-cluster effectively non-resonant for a given $\vec{\varkappa}$. Note, that the above estimate and, therefore, the definition of an effectively non-resonant $k^{\delta}$-cluster is stable in the $k^{-4 \gamma r_{1}^{\prime}-1-\delta}$-neighborhood of a given $\vec{\varkappa}$. The $k^{\delta}$-clusters, where (388) is not valid, are called effectively resonant $k^{\delta}$-clusters. Around each effectively resonant $k^{\delta}$-cluster, we construct $k^{\gamma r_{1}}$-clusters. We sort these clusters into four types: simple, white, grey and black clusters as in Section 4.4.1, using the term " $\mathrm{m}$ is effectively resonant" instead of "m $\in \mathcal{M}^{(2) "}$. There is no need to consider a special case of simple clusters here. Note that Lemmas $4.19-4.21$ are valid for an arbitrary $\vec{\varkappa}$, since they are based on Lemmas 4.8, 4.10, 4.12, 4.13, 4.16 proven for an arbitrary $\vec{\varkappa}$. Be analogy with (274), a $k^{\gamma r_{1}}$-cluster is called effectively non-resonant if

$$
\left\|\left(P(\mathbf{m})\left(H(\vec{\varkappa})-k^{2}\right) P(\mathbf{m})\right)^{-1}\right\|<k^{2 \mu r_{2}+r_{2}^{\prime} N_{i}^{(1)}},
$$

where $N_{i}^{(1)}$ corresponds to the color of a $k^{\gamma r_{1}}$-cluster, $N_{i}^{(1)}=k^{\gamma r_{1}+3}, k^{\gamma r_{1} / 2+\delta_{0} r_{1}}$ or $k^{\gamma r_{1} / 6-\delta_{0} r_{1}}$. If $n=4$ we stop here. If $n>4$, we surround effectively resonant $k^{\gamma r_{1}}$-clusters by blocks of the next size, etc. The analogues of Lemmas 4.19 - 4.21 are valid, see Lemmas 5.16 5.18, 7.7- 7.9, Eventually, the $k^{\gamma r_{n-3}}$-cluster is effectively non-resonant if

$$
\left\|\left(P(\mathbf{m})\left(H(\vec{\varkappa})-k^{2}\right) P(\mathbf{m})\right)^{-1}\right\|<k^{2 \mu r_{n-2}+r_{n-2}^{\prime} N_{i}^{(n-3)}}
$$

where $N_{i}^{(n-3)}$ is $N_{i}^{(n-3)}=k^{\gamma r_{n-3}+150 \gamma r_{n-4}}, k^{\gamma r_{n-3} / 2+\delta_{0} r_{n-3}}, k^{\gamma r_{n-3} / 6-\delta_{0} r_{n-3}}$, depending on the color of the cluster (cf. (389), (361) ). Further we put $150 \gamma r_{0}=3$. This will make (389) to be a special case of (390) $(n=4)$. Thus, we have constructed a block structure in $H^{(n-1)}(\vec{\varkappa})$, which is stable in the $k^{-\rho_{n-2}}$-neighborhood of a given $\vec{\varkappa}$, where $\rho_{1}=4 \gamma r_{1}^{\prime}+1+\delta$ and

$$
\rho_{n-2}=\mu r_{n-2}+r_{n-2}^{\prime} k^{\gamma r_{n-3}+150 \gamma r_{n-4}}+1+\delta, \text { when } n \geq 4 .
$$

It is not difficult to see that $\rho_{n-2}<r_{n-1}$.

Definition 7.1. We denote by $J(\vec{\varkappa})$ the number of the effectively resonant $k^{\gamma r_{n-3}}$-clusters in $H^{(n-1)}(\vec{\varkappa})$ for a given $\vec{\varkappa}$. Further (with a slight abuse of notations) we consider $J(\vec{\varkappa})$ to be constant in the $k^{-\rho_{n-2}}$-neighborhood of a given $\vec{\varkappa}$.

Let $\vec{\varkappa}=\mathbf{a} \tau_{1}+\mathbf{b}, \quad|\mathbf{a}|=1, \quad|\mathbf{b}|<4 k^{\gamma r_{n-2}}$. We consider $H^{(n-1)}(\vec{\varkappa})$ as a function of $\tau_{1}$ in the complex $k^{-\rho_{n-2}}$-neighbothood of zero. 
Lemma 7.2. The resolvent $\left(H^{(n-1)}(\vec{\varkappa})-k^{2}\right)^{-1}$ has no more than $k^{2 \gamma r_{n-3}} J(\mathbf{b})$ poles $\tau_{1 j}$ in the the complex $2 k^{-\rho_{n-2}}$-neighborhood of zero. It satisfies the following estimate in the the complex $k^{-\rho_{n-2}-n e i g h b o r h o o d ~ o f ~ z e r o: ~}$

$$
\left\|\left(H^{(n-1)}(\overrightarrow{\mathcal{\varkappa}})-k^{2}\right)^{-1}\right\|<k^{\rho_{n-2} k^{2 \gamma r_{n-3}}}\left(\frac{k^{-\rho_{n-2}}}{\varepsilon_{0}}\right)^{J(\vec{\varkappa}) k^{2 \gamma r_{n-3}}},
$$

where $\varepsilon_{0}=\min \left\{k^{-2 \rho_{n-2}}, \varepsilon\right\}$, $\varepsilon$ being the distance to the nearest pole $\tau_{1, j}$.

Proof. The lemma is proved by induction. For $n=3$, see Lemma 5.11. Let us consider the case $n \geq 4$. Recall (Definition 7.1) that $J(\vec{\varkappa})$ may be considered to be constant in $2 k^{-\rho_{n-2}}$-neighborhood of $\tau_{1}=0$. Hence, $J(\vec{\varkappa})=J(\mathbf{b})$ for such $\vec{\varkappa}$-s.

Let us consider the collection of all $k^{\delta}, \ldots, k^{\gamma r_{n-3}}$-clusters $P(\mathbf{m})$ for $H^{(n-1)}(\vec{\varkappa})$. Note that the collection is the same for all such $\vec{\varkappa}$. We construct the corresponding block operator $\tilde{H}^{(n-2)}(\vec{\varkappa})$ :

$$
\tilde{H}^{(n-2)}(\vec{\varkappa})=\sum P(\mathbf{m}) H P(\mathbf{m})+H_{0}\left(I-\sum P(\mathbf{m})\right) .
$$

If a $k^{\gamma r_{n-3}}$-cluster $P(\mathbf{m}) H(\vec{\varkappa}) P(\mathbf{m})$ is effectively non-resonant, then its resolvent, obvi-

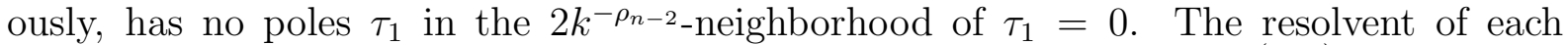
effectively resonant $k^{\gamma r_{n-3}}$-cluster $P(\mathbf{m}) H(\vec{\varkappa}) P(\mathbf{m})$ has no more than $N_{i}^{(n-3)} k^{2 \gamma r_{n-4}}\left(k^{2 \gamma r_{0}}\right.$ is taken to be equal to 12 for $n=4)$ poles $\tau_{1 j}$ in the $k^{-\rho_{n-3}}$-neighborhood of $\tau_{1}=0$. It follows from this lemma for the previous step and also Lemmas 4.19, 4.21, 5.16 5.18 and 7.7 7.9 below for previous steps, which give the estimates for $J(\vec{\varkappa})$ in the previous steps, based on the color of clusters. Let us consider the union of $k^{-2 \rho_{n-2}}$ neighborhoods of these poles and denote it by $\mathbf{D}_{\mathbf{m}}$. By this lemma for $n-1$, instead of $n$, each $k^{\gamma r_{n-3}}$ cluster satisfies the estimate

$$
\left\|\left(P(\mathbf{m})\left(H^{(n-1)}(\vec{\varkappa})-k^{2}\right) P(\mathbf{m})\right)^{-1}\right\|<k^{\rho_{n-3} k^{2 \gamma r_{n-4}}} k^{2 \rho_{n-2} N_{i}^{(n-3)} k^{2 \gamma r_{n-4}}}
$$

outside $\mathbf{D}_{\mathbf{m}}, N_{i}^{(n-3)}$ corresponding to the color of the cluster. Note that $\max _{i} N_{i}^{(n-3)}=$ $N_{1}^{(n-3)}<k^{\gamma r_{n-3}+150 \gamma r_{n-4}}$. Therefore, the resolvent $\left(\tilde{H}^{(n-2)}(\vec{\varkappa})-k^{2}\right)^{-1}$ has no more than $J(\vec{\varkappa}) N_{1}^{(n-3)} k^{2 \gamma r_{n-4}}$ poles $\tau_{1 j}$ in the complex $k^{-\rho_{n-3}}$-neighborhood of $\tau_{1}=0$. Let $\mathbf{D}=$ $\cup_{\mathbf{m}} \mathbf{D}_{\mathbf{m}}$, the union being taken over all $\mathbf{m}$ corresponding to all resonant clusters. The number of $\mathbf{m}$-s in the union, obviously, does not exceed $k^{4 \gamma r_{n-2}}$, which is the number of different $\mathbf{m}$ in $H^{(n-1)}(\vec{\varkappa})$. Therefore, the size of each connected component of $\mathbf{D}$ is less than $k^{-2 \rho_{n-2}} k^{4 \gamma r_{n-2}}=o\left(k^{-\rho_{n-2}}\right)$. We are interested only in those components of $\mathbf{D}$, which are completely in the disk of the radius $2 k^{-\rho_{n-2}}$ around $\tau_{1}=0$. Considering as before (see the proof of Theorem 5.1 with $r_{n-2}$ instead of $r_{2}, r_{n-3}$ instead of $r_{1}$ and $k^{\gamma r_{n-3}+150 \gamma r_{n-4}}$ instead of $k^{\gamma r_{1}+3}$, when one considers black clusters), we can show that the perturbation series for the resolvent $\left(H^{(n-1)}(\vec{\varkappa})-k^{2}\right)^{-1}$ with respect to $\left(\tilde{H}^{(n-2)}(\vec{\varkappa})-k^{2}\right)^{-1}$ converges on 
the boundary of $\mathbf{D}$. The resolvents have the same number of poles inside each component of D. Hence, $\left(H^{(n-1)}(\vec{\varkappa})-k^{2}\right)^{-1}$ has no more than $J(\vec{\varkappa}) N_{1}^{(n-3)} k^{2 \gamma r_{n-4}}$ poles in D. It is easy to see that $J(\vec{\varkappa}) N_{1}^{(n-3)} k^{2 \gamma r_{n-4}}<J(\vec{\varkappa}) k^{2 \gamma r_{n-3}}$. The resolvent satisfies the following estimate outside $\mathbf{D}$ :

$$
\left\|\left(H^{(n-1)}(\vec{\varkappa})-k^{2}\right)^{-1}\right\|<k^{\rho_{n-3} k^{2 \gamma r_{n-4}}} k^{2 \rho_{n-2} N_{1}^{(n-3)} k^{2 \gamma r_{n-4}}}<k^{\rho_{n-2} k^{2 \gamma r_{n-3}}} .
$$

Using the maximum principle we obtain (391).

Next, we introduce

$$
\mathcal{S}^{(n-1)}(k, \xi):=\left\{\vec{\varkappa} \in \mathbb{R}^{2}:\left\|\left(H^{(n-1)}(\vec{\varkappa})-k^{2}\right)^{-1}\right\|>k^{\xi}\right\}
$$

It is easy to see that each connected component of $\mathcal{S}^{(n-1)}(k, \xi)$ is bounded by the curves $D\left(\vec{\varkappa}, k^{2} \pm k^{-\xi}\right)=0$, where $D(\vec{\varkappa}, \lambda)=\operatorname{det}\left(H^{(n-1)}(\vec{\varkappa})-\lambda\right)$.

Lemma 7.3. Let $\mathbf{l}$ be a segment of a straight line in $\mathbb{R}^{2}$,

$$
\left.\mathbf{l}:=\left\{\vec{\varkappa}=\mathbf{a} \tau_{1}+\mathbf{b}, \tau_{1} \in(0, \eta)\right\}, \quad|\mathbf{a}|=1,|\mathbf{b}|<4 k^{\gamma r_{n-2}}, \quad 0<\eta<k^{-\rho_{n-2}}\right\} .
$$

Suppose both ends of $\mathbf{1}$ belong to a connected component of $\mathcal{S}^{(n-1)}(k, \xi)$. If $\xi$ is sufficiently large, namely, $\xi \geq 4 k^{2 \gamma r_{n-3}} J(\mathbf{b}) \log _{k} \frac{1}{\eta}$, then, there is an inner part $\mathbf{l}^{\prime}$ of the segment, which is not in $\mathcal{S}^{(n-1)}(k, \xi)$.

Corollary 7.4. Let $\vec{\varkappa} \in \mathcal{S}^{(n-1)}(k, \xi)$ and $\xi>8 k^{2 \gamma r_{n-3}} J(\vec{\varkappa}) \rho_{n-2}$. Then the distance from $\vec{\varkappa}$ to the boundary of $\mathcal{S}^{(n-1)}(k, \xi)$ is less than $k^{-\tilde{\xi}}, \tilde{\xi}=\xi \frac{1}{4} k^{-2 \gamma r_{n-3}} J(\overrightarrow{\mathcal{\varkappa}})^{-1}$.

Proof of the corollary. Let us consider a segment of the length $\eta=k^{-\tilde{\xi}}$ starting at $\vec{\varkappa}$. By the statement of the lemma it intersects a boundary $D\left(\vec{\varkappa}, k^{2} \pm k^{-\xi}\right)=0$.

Proof. Choose $\varepsilon=\eta^{2}$. Using the hypothesis of the lemma, we obtain that the righthand side of (391) is less than $k^{\xi}$ outside the discs of radius $\varepsilon$ around the poles of the resolvent. Let us estimate the total size (sum of the sizes) of the discs. Indeed, the size of each disc is $2 \eta^{2}$ and the number of discs is, obviously, less $16 k^{4 \gamma r_{n-2}}$. Therefore, the total size admits the estimate from above: $32 \eta^{2} k^{4 \gamma r_{n-2}}=o(\eta)$, since $\eta<k^{-\rho_{n-2}}$. This means there is a part $\mathbf{l}^{\prime}$ of $\mathbf{l}$ outside these discs. By (391), this part is outside $\mathcal{S}^{(n-1)}(k, \xi)$, when $\xi$ is as described in the statement of the lemma.

Let $\vec{\varkappa}_{0} \in \mathbb{R}^{2}$ be fixed and $\mathcal{N}\left(k, r_{n-1}, \vec{\varkappa}_{0}, J_{0}\right)$ be the following subset of the lattice $\vec{\varkappa}_{0}+\vec{p}_{\mathbf{n}}, \mathbf{n} \in \Omega\left(r_{n-1}\right):$

$$
\mathcal{N}\left(k, r_{n-1}, \vec{\varkappa}_{0}, J_{0}\right)=\left\{\vec{\varkappa}_{0}+\vec{p}_{\mathbf{n}}: \mathbf{n} \in \Omega\left(r_{n-1}\right): J\left(\vec{\varkappa}_{0}+\vec{p}_{\mathbf{n}}\right) \leq J_{0}\right\}
$$

$J$ being defined by Definition [7.1. Thus, $\mathcal{N}$ includes only such $\mathbf{n}$ that the surrounding $k^{\gamma r_{n-2}}$ - block contains less than $J_{0}$ of effectively resonant $k^{\gamma r_{n-3}}$-clusters. Let $N\left(k, r_{n-1}, \vec{\varkappa}_{0}, J_{0}, \xi\right)$ be the number of points $\vec{\varkappa}_{0}+\vec{p}_{\mathbf{n}}$ in $\mathcal{S}^{(n-1)}(k, \xi) \cap \mathcal{N}\left(k, r_{n-1}, \vec{\varkappa}_{0}, J_{0}\right)$. 
Lemma 7.5. If $\xi>4 \mu r_{n-1} J_{0} k^{2 \gamma r_{n-3}}$, then

$$
N\left(k, r_{n-1}, \vec{\varkappa}_{0}, J_{0}, \xi\right) \leq k^{\frac{2}{3} r_{n-1}+43 \gamma r_{n-2}} .
$$

Proof. The proof of the lemma is completely analogous to that of 5.14 up to replacement of 2 by $n-1$. Instead of Corollary 5.13 we use Corollary 7.4 and the inequality $\rho_{n-2}<r_{n-1}$. प

\subsubsection{Model Operator for Step $n+1$}

We make for $r_{n}$ the construction analogous to those from subsections 4.4.1, 5.3.2. We start with introducing a new notation by analogy with (345) and (350):

$$
\Omega_{s}^{(j)}\left(r_{n}\right)=\left\{\mathbf{m} \in \Omega\left(r_{n}\right), 0<p_{\mathbf{m}}<k^{-r_{j-1}^{\prime} k^{2 \gamma r_{j-2}}}\right\}, \quad j \geq 2,
$$

where $k^{2 \gamma r_{j-2}}$ is taken to be just 5 when $j=2$. Note that $\Omega_{s}^{(j+1)} \subset \Omega_{s}^{(j)}$ and $\Omega_{s}^{(j)}=\emptyset$ when $j>n$. Next, let $\mathbf{m} \in \Omega\left(r_{n}\right)$. We denote the $k^{\gamma r_{n-2}}$-component containing $\mathbf{m}$ by $\tilde{\Pi}(\mathbf{m})$ and the corresponding projector by $\tilde{P}(\mathbf{m})$ (we assume they are defined by the previous steps of the procedure). For $\mathbf{m}$ belonging to the same $k^{\gamma r_{n-2}}$-component, $\tilde{\Pi}(\mathbf{m})$ and $\tilde{P}(\mathbf{m})$ are the same. We define $\mathcal{M}^{(n)}$ by a recurrent formula, which starts with $\mathcal{M}^{(3)}$, see (344):

$\mathcal{M}^{(n)}:=\mathcal{M}^{(n)}\left(\varphi_{0}, r_{n}\right)=\left\{\mathbf{m} \in \mathcal{M}^{(n-1)}\left(\varphi_{0}, r_{n}\right) \cup \Omega_{s}^{(n-1)}\left(r_{n}\right) \backslash \mathcal{M}_{2}^{\text {weak }}\left(\varphi_{0}, r_{n}\right): \varphi_{0} \in \mathcal{O}_{\mathbf{m}}^{(n)}\left(r_{n-1}^{\prime}, 1\right)\right\}$,

where $\mathcal{O}_{\mathbf{m}}^{(n)}\left(r_{n-1}^{\prime}, \tau\right)$ is the union of the disks of the radius $\tau k^{-r_{n-1}^{\prime}}$ with the centers at poles of the resolvent $\left(\tilde{P}(\mathbf{m})\left(H\left(\vec{\varkappa}^{(n-1)}(\varphi)\right)-k^{2} I\right) \tilde{P}(\mathbf{m})\right)^{-1}$ in the $k^{-44 r_{n-2}^{\prime}-2-\delta}$-neighborhood of $\varphi_{0}$. For $\mathbf{m}$ belonging to the same $k^{\gamma r_{n-2}}$-component, the sets $\mathcal{O}_{\mathbf{m}}^{(n)}\left(r_{n-1}^{\prime}, \tau\right)$ are identical. We say that $\mathbf{m} \in \mathcal{M}^{(n)}$ is $k^{\gamma r_{n-2}}$-resonant. The corresponding $k^{\gamma r_{n-2}}$-clusters we call resonant too.

Let $\varphi_{0} \in \omega^{(n)}(k, \delta, 1)$. By construction of the non-resonant set $\omega^{(n)}(k, \delta, 1)$, we have $\mathcal{M}^{(n)} \cap \Omega\left(r_{n-1}\right)=\emptyset$.

Further we use the property of the set $\mathcal{M}^{(n)}$ formulated in the next lemma which is an analogue of the Lemmas 4.18, 5.15.

Lemma 7.6. Let $r_{n-1}^{\prime}>2 k^{\left(\gamma+\delta_{0}\right) 10^{-4} r_{n-2}-2 \delta}$. Let $1 / 20<\gamma^{\prime}<20, \mathbf{m}_{0} \in \Omega\left(r_{n}\right)$ and $\Pi_{\mathbf{m}_{0}}$ be the $k^{\gamma^{\prime} r_{n-1}}$-neighborhood (in $\left\||\cdot \||\right.$-norm) of $\mathbf{m}_{0}$. Then the set $\Pi_{\mathbf{m}_{0}}$ contains less than $k^{\frac{2}{3} \gamma^{\prime} r_{n-1}+50 \gamma r_{n-2}}$ elements of $\mathcal{M}^{(n)}$.

Proof. The proof is similar to that of Lemma 5.15 up to the replacement of 3 by $n$. First, we notice that the condition $r_{n-1}^{\prime}>2 k^{\left(\gamma+\delta_{0}\right) 10^{-4} r_{n-2}-2 \delta}$ is consistent with the restriction (384). Instead of Lemma 3.31 we use Lemma $4.28(n=4)$, Lemma 5.23 $(n=5)$ and Lemma 7.14 with $n-2$ instead of $n$ when $n>5$. We also use Lemma 7.5 instead of Lemma 5.14. We use (384) to show that the hypothesis of Lemma 7.5 holds. In particular, we use the inequality $r_{n-1}^{\prime}>>4 \mu r_{n-1} k^{2 \gamma r_{n-3}}$, following from (384). 
We continue with constructing $k^{\gamma r_{n-2}}$-clusters in $\Omega\left(r_{n}\right), r_{n}>r_{n-1}$, the same way we did it for $\Omega\left(r_{2}\right)$ in Section 4.4.1. We call a $k^{\gamma r_{n-2}}$-cluster resonant if it contains $\mathbf{m} \in \mathcal{M}^{(n)}$, see (344), (396). Next, we repeat the construction after Lemma 4.18 up to the replacement

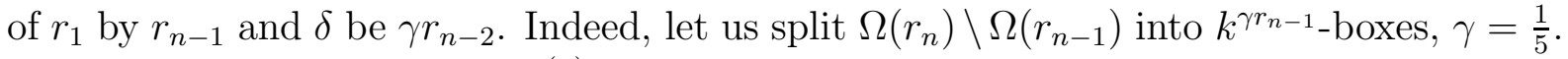

First, let's consider $\mathbf{m} \in \Omega_{s}^{(n)}\left(r_{n}\right)$. As before (see "Simple region", page 67) one can prove that $\Omega_{s}^{(n)}\left(r_{n}\right) \subset \mathcal{M}\left(r_{n}\right)$; there are no other elements of $\mathcal{M}\left(r_{n}\right)$ in the $k^{\delta}$-box around $\mathbf{m} ; \mathbf{m}$ itself can belong or do not belong to $\mathcal{M}^{(j)}\left(r_{n}\right)$, but there are no other elements of $\mathcal{M}^{(j)}\left(r_{n}\right)$ in the $k^{r_{j-1}}$-box around such $\mathbf{m}, j=2, \ldots, n$; and there are no other elements of $\Omega_{s}^{(n)}\left(r_{n}\right)$ in the $k^{r_{n-1}}$-box around $\mathbf{m}$.

For each $\mathbf{m} \in \Omega_{s}^{(n)}\left(r_{n}\right)$ we consider its $k^{r_{n-1} / 2}$-neighborhood in $\||\cdot \||$ norm. The union of such boxes we call the simple region and denote $\Pi_{s}$. The corresponding projection is $P_{s}$.

Now, consider all other boxes (all elements $\vec{p}_{\mathbf{m}}$ there satisfy $p_{\mathbf{m}}>k^{-r_{n-1}^{\prime} k^{2 \gamma r_{n-2}}}$ ). We call a box black if it together with its neighbors contains more than $k^{\gamma r_{n-1} / 2+\delta_{0} r_{n-1}}$ elements of $\mathcal{M}^{(n)}, \delta_{0}=\gamma / 100$. Let us consider "black" boxes together with their $k^{\gamma r_{n-1}+\delta_{0} r_{n-1}}$ neighborhoods and call this the black region. We denote the black region by $\Pi_{b}$. The corresponding projector is $P_{b}$. By white boxes we mean $k^{\gamma r_{n-1}}$-boxes which together with its neighbors contain no more than $k^{\gamma r_{n-1} / 2+\delta_{0} r_{n-1}}$ elements of $\mathcal{M}^{(n)}$. Every white box we split into "small" boxes of the size $k^{\gamma r_{n-1} / 2+2 \delta_{0} r_{n-1}}$. We call a small box "grey" if it together with its neighbors contains more than $k^{\gamma r_{n-1} / 6-\delta_{0} r_{n-1}}$ elements of $\mathcal{M}^{(n)}$. Grey small boxes together with its $k^{\gamma r_{n-1} / 2+2 \delta_{0} r_{n-1}}$-neighborhoods we call the grey region. The notation for this region is $\Pi_{g}$. The corresponding projector is $P_{g}$. The part of the grey region which is outside the black region, we denote $\Pi_{g}^{\prime}$ and the corresponding projection by $P_{g}^{\prime}$. By a white small box we call a small box which has no more than $k^{\gamma r_{n-1} / 6-\delta_{0} r_{n-1}}$ elements of $\mathcal{M}^{(n)}$. In each small white box we consider $k^{\gamma r_{n-1} / 6}$-boxes around each point of $\mathcal{M}^{(n)}$. The union of such $k^{\gamma r_{n-1} / 6}$-boxes we call the white region and denote $\Pi_{w}$. The corresponding projection is $P_{w}$. The part of the white region which is outside the black and grey regions, we denote $\Pi_{w}^{\prime}$ and the corresponding projection by $P_{w}^{\prime}$.

We put as before

$$
P_{r}^{(n)}:=P_{s}^{(n)}+P_{b}^{(n)}+P_{g}^{(n)^{\prime}}+P_{w}^{(n)^{\prime}} .
$$

The construction of the non-resonant region is the inductive extension of that for Step IV, see Section 5.3, page [96. Indeed, we start with construction of $k^{\delta}$ clusters in $\Omega\left(r_{n}\right)$. Those of them, which are resonant, we extend to $k^{\gamma r_{1}}$ clusters, those of them, which are resonant we extend to $k^{\gamma r_{2}}$ clusters, and so on until we reach the size $k^{\gamma r_{n-2}}$. On each step we construct a colored structure (simple, black, grey, white). If $k^{\gamma r_{j}}$-cluster happens to intersect $k^{\gamma r_{j+1}}$-cluster, we consider it to be a part of $k^{\gamma r_{j+1}}$-cluster. Thus, $k^{\delta}$-clusters are built around $\mathcal{M}\left(r_{n}, \varphi_{0}\right) \backslash\left(\mathcal{M}\left(r_{n-1}, \varphi_{0}\right) \cup \mathcal{M}^{(2)}\left(r_{n}, \varphi_{0}\right) \cup \Omega_{s}^{(2)}\left(r_{n}\right) \cup \mathcal{M}_{2, t w}\left(r_{n}, \varphi_{0}\right)\right)$. The union of this neighborhoods we denote $\Pi_{n r, \delta}$. Next, $k^{\gamma r_{j}}$-clusters $(j \geq 1)$ are built around the points of $\mathcal{M}^{(j+1)}\left(r_{n}, \varphi_{0}\right) \cup \Omega_{s}^{(j+1)}\left(r_{n}\right) \backslash\left(\mathcal{M}^{(j+1)}\left(r_{n-1}, \varphi_{0}\right) \cup \mathcal{M}^{(j+2)}\left(r_{n}, \varphi_{0}\right) \cup \Omega_{s}^{(j+2)}\left(r_{n}\right)\right)$. 
The set of all non-resonant $k^{\gamma r_{j}}$-clusters we denote by $\Pi_{n r, r_{j}}^{(n)}$. It is convenient to identify $\Pi_{n r, r_{0}}^{(n)}:=\Pi_{n r, \delta}^{(n)}$. Then

$$
\Pi_{n r}^{(n)}:=\cup_{j=0}^{n-2} \Pi_{n r, r_{j}}^{(n)},
$$

Those $\Pi_{n r, r_{j}}^{(n)}$, which intersect with $\Pi_{r}^{(n)}$ we attach to $\Pi_{r}^{(n)}$ just slightly abusing the notation (cf. Section 5.3). The part of $\Pi_{n r, r_{j}}^{(n)}$ which does not intersect with $\Pi_{r}^{(n)}$ we denote by $\Pi_{n r, r_{j}}^{(n)^{\prime}}$. Correspondingly, the part of $\Pi_{n r}^{(n)}$ which does not intersect $\Pi_{r}^{(n)}$ is denoted by $\Pi_{n r}^{(n)^{\prime}}$. Further,

$$
P^{(n)}:=P_{r}^{(n)}+P_{n r}^{(n)^{\prime}}+P\left(r_{n-1}\right) .
$$

We continue construction from Section 4.4. Repeating the arguments from the proofs of Lemmas 4.19, 4.20, 4.21 with obvious changes (in particular, using Lemma 7.6 instead of Lemmas 4.18, 5.15) we obtain the following results. (Here and in what follows we will omit superscript $(n)$ when it does not lead to a confusion.)

Lemma 7.7. 1. Each $\Pi_{b}^{j}$ contains no more than $k^{\gamma r_{n-1} / 2-\delta_{0} r_{n-1}+150 \gamma r_{n-2}}$ black boxes.

2. The size of $\Pi_{b}^{j}$ in $\left\||\cdot \||\right.$ norm is less than $k^{3 \gamma r_{n-1} / 2+150 \gamma r_{n-2}}$.

3. Each $\Pi_{b}^{j}$ contains no more than $k^{\gamma r_{n-1}+150 \gamma r_{n-2}}$ elements of $\mathcal{M}^{(n)}$. Moreover, any box of $\left\||\cdot \||\right.$-size $k^{3 \gamma r_{n-1} / 2+150 \gamma r_{n-2}}$ containing $\Pi_{b}^{j}$ has no more than $k^{\gamma r_{n-1}+150 \gamma r_{n-2}}$ elements of $\mathcal{M}^{(n)}$ inside.

Lemma 7.8. 1. Each $\Pi_{g}^{j}$ contains no more than $k^{\gamma r_{n-1} / 3+2 \delta_{0} r_{n-1}}$ grey boxes.

2. The size of $\Pi_{g}^{j}$ in $\left\||\cdot \||\right.$ norm is less than $k^{5 \gamma r_{n-1} / 6+4 \delta_{0} r_{n-1}}$.

3. Each $\Pi_{g}^{j}$ contains no more than $k^{\gamma r_{n-1} / 2+\delta_{0} r_{n-1}}$ elements of $\mathcal{M}^{(n)}$.

Lemma 7.9. 1. The size of $\Pi_{w}^{j}$ in \|\|$\cdot \|$ norm is less than $k^{\gamma r_{n-1} / 3-\delta_{0} r_{n-1}}$.

2. Each $\Pi_{w}^{j}$ contains no more than $k^{\gamma r_{n-1} / 6-\delta_{0} r_{n-1}}$ points of $\mathcal{M}^{(n)}$.

The construction of the rest of Section 4.4.1 stays unchanged. Let us introduce corresponding notation, formulate the results and provide some comments.

Next lemmas are the analogues of Lemmas 4.24, 4.25, 4.26.

Lemma 7.10. Let $\varphi_{0} \in \omega^{(n)}(k, \delta, \tau),\left|\varphi-\varphi_{0}\right|<k^{-k^{r_{n}-2}}$. Then,

$$
\left\|\left(P_{n r}\left(H\left(\vec{\varkappa}^{(n)}(\varphi)\right)-k^{2} I\right) P_{n r}\right)^{-1}\right\|<k^{r_{n-1}^{\prime} k^{2 \gamma r_{n-2}}} k^{r_{n-1}^{\prime}} \leq k^{k^{3 \gamma r_{n-2}}} .
$$

Lemma 7.11. Let $\varphi_{0} \in \omega^{(n)}(k, \delta, \tau)$, and $\left|\varphi-\varphi_{0}\right|<k^{-k^{r_{n}-2}}, i=1,2,3$. Then, 
1. The number of poles of the resolvent $\left(P_{i}\left(H\left(\vec{\varkappa}^{(n)}(\varphi)\right)-k^{2} I\right) P_{i}\right)^{-1}$ in the disc $\mid \varphi-$ $\varphi_{0} \mid<k^{-k^{r_{n-2}}}$ is no greater than $N_{i}^{(n-1)}$, where $N_{1}^{(n-1)}=k^{\gamma r_{n-1}+150 \gamma r_{n-2}}, N_{2}^{(n-1)}=$ $k^{\gamma r_{n-1} / 2+\delta_{0} r_{n-1}}, N_{3}^{(n-1)}=k^{\gamma r_{n-1} / 6-\delta_{0} r_{n-1}}$.

2. Let $\varepsilon$ be the distance to the nearest pole of the resolvent in $\mathcal{W}^{(n)}$ and let $\varepsilon_{0}=$ $\min \left\{\varepsilon, k^{-r_{n-1}^{\prime}}\right\}$. Then the following estimates hold:

$$
\begin{gathered}
\left\|\left(P_{i}\left(H\left(\vec{\varkappa}^{(n)}(\varphi)\right)-k^{2} I\right) P_{i}\right)^{-1}\right\|<k^{2 r_{n-1}^{\prime} k^{2 \gamma r_{n-2}}} k^{r_{n-1}^{\prime}}\left(\frac{k^{-r_{n-1}^{\prime}}}{\varepsilon_{0}}\right)^{N_{i}^{(n-1)}} \leq \\
k^{k^{3 \gamma r_{n-2}}}\left(\frac{k^{-r_{n-1}^{\prime}}}{\varepsilon_{0}}\right)^{N_{i}^{(n-1)}}, \\
\left\|\left(P_{i}\left(H\left(\vec{\varkappa}^{(n)}(\varphi)\right)-k^{2} I\right) P_{i}\right)^{-1}\right\|_{1}<k^{2 r_{n-1}^{\prime} k^{2 \gamma r_{n-2}}} k^{r_{n-1}^{\prime}+8 \gamma r_{n-1}}\left(\frac{k^{-r_{n-1}^{\prime}}}{\varepsilon_{0}}\right)^{N_{i}^{(n-1)}} \leq \\
k^{k^{3 \gamma r_{n-2}}}\left(\frac{k^{-r_{n-1}^{\prime}}}{\varepsilon_{0}}\right)^{N_{i}^{(n-1)}} .
\end{gathered}
$$

Proof. The proof of this lemma is analogous to that of Lemma 4.25 up to the replacement of $\mathcal{M}^{(2)}$ by $\mathcal{M}^{(n)}, \mathcal{O}_{\mathbf{m}}^{(2)}$ by $\mathcal{O}_{\mathbf{m}}^{(n)}$, and the shift of indices: $\delta$ to $r_{n-2}, r_{1}$ to $r_{n-1}$, etc. We apply Lemmas $7.7 \sqrt{7.9}$ instead of 4.19 4.21. We apply Lemmas [5.20, 5.21] with $\varepsilon_{0}=k^{-r_{3}^{\prime}}$ and $p_{\mathbf{m}}>k^{-r_{3}^{\prime} k^{2 \gamma r_{2}}}$ instead of Lemma 4.25, 4.26 for $n=4$ and Lemmas 17.11, 7.12 with inductively (with $n-1$ instead of $n$ and $\varepsilon_{0}=k^{-r_{n-1}^{\prime}}, p_{\mathbf{m}}>k^{-r_{n-1}^{\prime} k^{2 \gamma r_{n-2}^{\prime}}}$ ) for further steps.

Let $\Pi_{s}^{j}$ be a particular $k^{r_{n-1} / 2}$-box around $\mathbf{m} \in \Omega_{s}^{(n)}\left(r_{n}\right)$ and let $P_{s}^{j}$ be the corresponding projection.

Lemma 7.12. Let $\varphi_{0} \in \omega^{(n)}(k, \delta, \tau)$. Then, the operator $\left(P_{s}^{j}\left(H\left(\vec{\varkappa}^{(n)}(\varphi)\right)-k^{2} I\right) P_{s}^{j}\right)^{-1}$ has no more than one pole in the disk $\left|\varphi-\varphi_{0}\right|<k^{-k^{r} n-2}$. Moreover,

$$
\begin{gathered}
\left\|\left(P_{s}^{j}\left(H\left(\vec{\varkappa}^{(n)}(\varphi)\right)-k^{2} I\right) P_{s}^{j}\right)^{-1}\right\|<\frac{8 k^{-1}}{p_{\mathbf{m}} \varepsilon_{0}}, \\
\left\|\left(P_{s}^{j}\left(H\left(\vec{\varkappa}^{(n)}(\varphi)\right)-k^{2} I\right) P_{s}^{j}\right)^{-1}\right\|_{1}<\frac{8 k^{-1+4 r_{n-1}}}{p_{\mathbf{m}} \varepsilon_{0}},
\end{gathered}
$$

$\varepsilon_{0}=\min \left\{\varepsilon, k^{-r_{n-1}^{\prime}}\right\}$, where $\varepsilon$ is the distance to the pole of the operator.

Note that $p_{\mathbf{m}}>k^{-2 \mu r_{n}}$ when $\mathbf{m} \in \Omega\left(r_{n}\right)$. The analogues of Lemma 4.22 and Corollary 4.23 also hold. 


\subsubsection{Resonant and Nonresonant Sets for Step $n+1$}

We divide $[0,2 \pi)$ into $\left[2 \pi k^{k^{r} n-2}\right]+1$ intervals $\Delta_{l}^{(n)}$ with the length not bigger than $k^{-k^{r_{n-2}}}$. If a particular interval belongs to $\mathcal{O}^{(n)}$ we ignore it; otherwise, let $\varphi_{0}^{(l)} \notin \mathcal{O}^{(n)}$ be a point inside the $\Delta_{l}^{(n)}$. Let

$$
\mathcal{W}_{l}^{(n)}=\left\{\varphi \in \mathcal{W}^{(n)}:\left|\varphi-\varphi_{0}^{(l)}\right|<4 k^{-k^{r_{n}-2}}\right\}
$$

Clearly, neighboring sets $\mathcal{W}_{l}^{(n)}$ overlap (because of the multiplier 4 in the inequality), they cover $\hat{\mathcal{W}}^{(n)}$, which is the restriction of $\mathcal{W}^{(n)}$ to the $2 k^{-k^{r} n-2}$-neighborhood of $[0,2 \pi)$. For each $\varphi \in \hat{\mathcal{W}}^{(n)}$ there is an $l$ such that $\left|\varphi-\varphi_{0}^{(l)}\right|<4 k^{-k^{r_{n}-2}}$. We consider the poles of the resolvent $\left(P^{(n)}\left(H\left(\vec{\varkappa}^{(n)}(\varphi)\right)-k^{2}\right) P^{(n)}\right)^{-1}$ in $\hat{\mathcal{W}}_{l}^{(n)}$ and denote them by $\varphi_{l m}^{(n)}, l=1, \ldots, M_{m}$. As before, the resolvent has a block structure. The number of blocks clearly cannot exceed the number of elements in $\Omega\left(r_{n}\right)$, i.e. $k^{4 r_{n}}$. Using the estimates for the number of poles for each block, the estimate being provided by Lemma 7.11 Part 1, we can roughly estimate the number of poles of the resolvent by $k^{4 r_{n}+r_{n-1}}$. Next, let $\mathcal{O}_{l m}^{(n+1)}$ be the disc of the radius $k^{-r_{n}^{\prime}}$ around $\varphi_{l m}^{(n)}$.

Definition 7.13. The set

$$
\mathcal{O}^{(n+1)}=\cup_{l m} \mathcal{O}_{l m}^{(n+1)}
$$

we call the $n+1$-th resonant set. The set

$$
\mathcal{W}^{(n+1)}=\mathcal{W}^{(n)} \backslash \mathcal{O}^{(n+1)}
$$

is called the $n+1$-th non-resonant set. The set

$$
\omega^{(n+1)}=\mathcal{W}^{(n+1)} \cap[0,2 \pi)
$$

is called the $n+1$-th real non-resonant set.

The following statements can be proven in the same way as Lemmas 4.28, 4.29 and 4.30.

Lemma 7.14. Let $r_{n}^{\prime}>\mu r_{n}>k^{r_{n-2}}, \varphi \in \mathcal{W}^{(n+1)}, \varphi_{0}(m)$ corresponds to an interval $\Delta_{l}^{(n)}$ containing $\Re \varphi$. Let $\Pi$ be one of the components $\Pi_{s}^{j}\left(\varphi_{0}^{(l)}\right), \Pi_{b}^{j}\left(\varphi_{0}^{(l)}\right), \Pi_{g}^{j}\left(\varphi_{0}^{(l)}\right), \Pi_{w}^{j}\left(\varphi_{0}^{(l)}\right)$ and $P(\Pi)$ be the projection corresponding to $\Pi$. Let also $\varkappa \in \mathbb{C}:\left|\varkappa-\varkappa^{(n)}(\varphi)\right|<k^{-r_{n}^{\prime} k^{2 \gamma r_{n-1}}}$. Then,

$$
\begin{gathered}
\left\|\left(P(\Pi)\left(H(\vec{\varkappa}(\varphi))-k^{2} I\right) P(\Pi)\right)^{-1}\right\|<k^{2 \mu r_{n}+r_{n}^{\prime} N^{(n-1)}}, \\
\left\|\left(P(\Pi)\left(H(\vec{\varkappa}(\varphi))-k^{2} I\right) P(\Pi)\right)^{-1}\right\|_{1}<k^{(2 \mu+1) r_{n}+r_{n}^{\prime} N^{(n-1)}},
\end{gathered}
$$

$N^{(n-1)}$ corresponding to the color of $\Pi\left(N^{(n-1)}=1, k^{\gamma r_{n-1}+150 \gamma r_{n-2}}, k^{\gamma r_{n-1} / 2+\delta_{0} r_{n-1}}\right.$, $k^{\gamma r_{n-1} / 6-\delta_{0} r_{n-1}}$ for simple, black, grey and white clusters, correspondingly). 
Proof. The lemma follows from Lemmas 7.11, 7.12 and the definition of $\mathcal{W}^{(n+1)}$.

We also notice that inequalities $r_{n}^{\prime}>\mu r_{n}>k^{r_{n-2}}$ follow from (384). By total size of the set $\mathcal{O}^{(n+1)}$ we mean the sum of the sizes of its connected components.

Lemma 7.15. Let $r_{n}^{\prime} \geq(\mu+10) r_{n}, r_{n}>k^{r_{n-2}}$. Then, the size of each connected component of $\mathcal{O}^{(n+1)}$ is less than $32 k^{4 r_{n}-r_{n}^{\prime}}$. The total size of $\mathcal{O}^{(n+1)}$ is less than $k^{-r_{n}^{\prime} / 2}$.

Lemma 7.16. Let $\varphi \in \mathcal{W}^{(n)}$ and $C_{n+1}$ be the circle $\left|z-k^{2}\right|=k^{-2 r_{n}^{\prime} k^{2 \gamma r_{n-1}}}$. Then

$$
\left\|\left(P\left(r_{n-1}\right)\left(H\left(\vec{\varkappa}^{(n)}(\varphi)\right)-z\right) P\left(r_{n-1}\right)\right)^{-1}\right\| \leq 4^{n} k^{2 r_{n}^{\prime} k^{2 \gamma r_{n-1}}} .
$$

\subsection{Operator $H^{(n+1)}$. Perturbation Formulas}

Let $P\left(r_{n}\right)$ be an orthogonal projector onto $\Omega\left(r_{n}\right):=\left\{\mathbf{m}:\left\|\vec{p}_{\mathbf{m}}\right\| \mid \leq k^{r_{n}}\right\}$ and $H^{(n+1)}=$ $P\left(r_{n}\right) H P\left(r_{n}\right)$. We consider $H^{(n+1)}\left(\vec{\varkappa}^{(n)}(\varphi)\right)$ as a perturbation of

$$
\tilde{H}^{(n)}=\tilde{P}_{l}^{(n)} H \tilde{P}_{l}^{(n)}+\left(P\left(r_{n}\right)-\tilde{P}_{l}^{(n)}\right) H_{0},
$$

where $H=H\left(\vec{\varkappa}^{(n)}(\varphi)\right), H_{0}=H_{0}\left(\vec{\varkappa}^{(n)}(\varphi)\right)$, and $\tilde{P}_{l}^{(n)}$ is the projection $P^{(n)}$, see (397), corresponding to $\varphi_{0}^{(l)}$ in the interval $\Delta_{l}^{(n)}$ containing $\varphi$. Let

$$
\begin{gathered}
W^{(n)}=H^{(n+1)}-\tilde{H}^{(n)}=P\left(r_{n}\right) V P\left(r_{n}\right)-\tilde{P}_{l}^{(n)} V \tilde{P}_{l}^{(n)}, \\
g_{r}^{(n+1)}(\vec{\varkappa}):=\frac{(-1)^{r}}{2 \pi i r} \operatorname{Tr} \oint_{C_{n+1}}\left(W^{(n)}\left(\tilde{H}^{(n)}(\vec{\varkappa})-z I\right)^{-1}\right)^{r} d z, \\
G_{r}^{(n)}(\vec{\varkappa}):=\frac{(-1)^{r+1}}{2 \pi i} \oint_{C_{n+1}}\left(\tilde{H}^{(n)}(\vec{\varkappa})-z I\right)^{-1}\left(W^{(n)}\left(\tilde{H}^{(n)}(\vec{\varkappa})-z I\right)^{-1}\right)^{r} d z,
\end{gathered}
$$

where $C_{n+1}$ is the circle $\left|z-k^{2}\right|=\varepsilon_{0}^{(n+1)}, \varepsilon_{0}^{(n+1)}=k^{-2 r_{n}^{\prime} k^{2 \gamma r_{n-1}}}$.

Recall that $\beta:=\delta_{*} / 100$. The proof of the following statements is analogous to the one in Step III (see Theorem 5.1, Corollary 5.2, Lemma 5.3 and Lemma 5.5).

Theorem 7.17. Suppose $k>k_{*}, \varphi$ is in the real $k^{-r_{n}^{\prime}-\delta}$-neighborhood of $\omega^{(n+1)}(k, \delta, \tau)$ and $\varkappa \in \mathbb{R},\left|\varkappa-\varkappa^{(n)}(\varphi)\right| \leq \varepsilon_{0}^{(n+1)} k^{-1-\delta}, \vec{\varkappa}=\varkappa(\cos \varphi, \sin \varphi)$. Then, there exists a single eigenvalue of $H^{(n+1)}(\vec{\varkappa})$ in the interval $\varepsilon_{n+1}(k, \delta, \tau)=\left(k^{2}-\varepsilon_{0}^{(n+1)}, k^{2}+\varepsilon_{0}^{(n+1)}\right)$. It is given by the absolutely converging series series:

$$
\lambda^{(n+1)}(\vec{\varkappa})=\lambda^{(n)}(\vec{\varkappa})+\sum_{r=2}^{\infty} g_{r}^{(n+1)}(\vec{\varkappa}) .
$$

For coefficients $g_{r}^{(n+1)}(\vec{\varkappa})$ the following estimates hold:

$$
\left|g_{r}^{(n+1)}(\vec{\varkappa})\right|<k^{-\frac{\beta}{5} k^{r_{n-1}-r_{n-2}-\beta(r-1)}} .
$$


The corresponding spectral projection is given by the series:

$$
\mathcal{E}^{(n+1)}(\vec{\varkappa})=\mathcal{E}^{(n)}(\vec{\varkappa})+\sum_{r=1}^{\infty} G_{r}^{(n+1)}(\vec{\varkappa}),
$$

$\mathcal{E}^{(n)}(\vec{\varkappa})$ being the spectral projection of $H^{(n)}$. The operators $G_{r}^{(n+1)}(\vec{\varkappa})$ satisfy the estimates:

$$
\begin{gathered}
\left\|G_{r}^{(n+1)}(\overrightarrow{\mathcal{\varkappa}})\right\|_{1}<k^{-\frac{\beta}{10} k^{r_{n-1}-r_{n-2}-\beta r}} \\
G_{r}^{(n+1)}(\overrightarrow{\mathcal{\varkappa}})_{\mathbf{s s}^{\prime}}=0, \text { when } 2 r k^{\gamma r_{n-1}+150 \gamma r_{n-2}}+3 k^{r_{n-1}}<\left\|\mid \vec{p}_{\mathbf{s}}\right\|+\|\| \vec{p}_{\mathbf{s}^{\prime}} \| .
\end{gathered}
$$

Corollary 7.18. For the perturbed eigenvalue and its spectral projection the following estimates hold:

$$
\begin{gathered}
\lambda^{(n+1)}(\vec{\varkappa})=\lambda^{(n)}(\vec{\varkappa})+O_{2}\left(k^{-\frac{1}{5} \beta k^{r_{n-1}-r_{n-2}}}\right), \\
\left\|\mathcal{E}^{(n+1)}(\vec{\varkappa})-\mathcal{E}^{(n)}(\vec{\varkappa})\right\|_{1}<k^{-\frac{\beta}{10} k^{r_{n-1}-r_{n-2}},} \\
\left|\mathcal{E}^{(n+1)}(\vec{\varkappa})_{\mathbf{s s}^{\prime}}\right|<k^{-d^{(n+1)}\left(\mathbf{s}, \mathbf{s}^{\prime}\right)}, \text { when }\left\|\left|\vec{p}_{\mathbf{s}} \|\right|>4 k^{r_{n-1}} \text { or }\right\|\left|\vec{p}_{\mathbf{s}^{\prime}} \|\right|>4 k^{r_{n-1}}, \\
d^{(n+1)}\left(\mathbf{s}, \mathbf{s}^{\prime}\right)=\frac{1}{16}\left(\left\|\left|\vec { p } _ { \mathbf { s } } \left\|\left|+\left\|\left|\vec{p}_{\mathbf{s}^{\prime}} \|\right|\right) k^{-\gamma r_{n-1}-150 \gamma r_{n-2}} \beta+\frac{1}{10} \beta k^{r_{n-1}-r_{n-2}} .\right.\right.\right.\right.\right.
\end{gathered}
$$

Lemma 7.19. Under conditions of Theorem 7.17 the following estimates hold when $\varphi \in$ $\omega^{(n+1)}(k, \delta)$ or its complex $k^{-r_{n}^{\prime}-\delta}$ neighborhood and $\varkappa \in \mathbb{C},\left|\varkappa-\varkappa^{(n)}(\varphi)\right|<\varepsilon_{0}^{(n+1)} k^{-1-\delta}$.

$$
\begin{gathered}
\lambda^{(n+1)}(\vec{\varkappa})=\lambda^{(n)}(\vec{\varkappa})+O_{2}\left(k^{\left.-\frac{1}{5} \beta k^{r_{n-1}-r_{n-2}}\right)}\right. \\
\frac{\partial \lambda^{(n+1)}}{\partial \varkappa}=\frac{\partial \lambda^{(n)}}{\partial \varkappa}+O_{2}\left(k^{-\frac{1}{5} \beta k^{r_{n-1}-r_{n-2}}} M_{n-1}\right), \quad M_{n-1}:=\frac{k^{1+\delta}}{\varepsilon_{0}^{(n+1)}} \\
\frac{\partial \lambda^{(n+1)}}{\partial \varphi}=\frac{\partial \lambda^{(n)}}{\partial \varphi}+O_{2}\left(k^{\left.-\frac{1}{5} \beta k^{r_{n-1}-r_{n-2}+r_{n}^{\prime}+\delta}\right)}\right. \\
\frac{\partial^{2} \lambda^{(n+1)}}{\partial \varkappa^{2}}=\frac{\partial^{2} \lambda^{(n)}}{\partial \varkappa^{2}}+O_{2}\left(k^{-\frac{1}{5} \beta k^{r_{n-1}-r_{n-2}}} M_{n-1}^{2}\right) \\
\frac{\partial^{2} \lambda^{(n+1)}}{\partial \varkappa \partial \varphi}=\frac{\partial^{2} \lambda^{(n)}}{\partial \varkappa \partial \varphi}+O_{2}\left(k^{\left.-\frac{1}{5} \beta k^{r_{n-1}-r_{n-2}+r_{n}^{\prime}+\delta} M_{n-1}\right)}\right. \\
\frac{\partial^{2} \lambda^{(n+1)}}{\partial \varphi^{2}}=\frac{\partial^{2} \lambda^{(n)}}{\partial \varphi^{2}}+O_{2}\left(k^{\left.-\frac{1}{5} \beta k^{r_{n-1}-r_{n-2}+2 r_{n}^{\prime}+2 \delta}\right)} .\right.
\end{gathered}
$$

Corollary 7.20. All " $\mathrm{O}_{2}$ "-s on the right hand sides of (421)-(426) can be written as $O_{1}\left(k^{-\frac{1}{10} \beta k^{r_{n-1}-r_{n-2}}}\right)$. 


\subsection{Isoenergetic Surface for Operator $H^{(n+1)}$}

The following statement is an analogue of Lemma 5.5.

Lemma 7.21. 1. For every $\lambda:=k^{2}, k>k_{*}$, and $\varphi$ in the real $\frac{1}{2} k^{-r_{n}^{\prime}-\delta}$-neighborhood of $\omega^{(n+1)}(k, \delta, \tau)$, there is a unique $\varkappa^{(n+1)}(\lambda, \varphi)$ in the interval $I_{n}:=\left[\varkappa^{(n)}(\lambda, \varphi)-\right.$ $\left.\varepsilon_{0}^{(n+1)} k^{-1-\delta}, \varkappa^{(n)}(\lambda, \varphi)+\varepsilon_{0}^{(n+1)} k^{-1-\delta}\right]$, such that

$$
\lambda^{(n+1)}\left(\vec{\varkappa}^{(n+1)}(\lambda, \varphi)\right)=\lambda, \quad \vec{\varkappa}^{(n+1)}(\lambda, \varphi):=\varkappa^{(n+1)}(\lambda, \varphi) \vec{\nu}(\varphi) .
$$

2. Furthermore, there exists an analytic in $\varphi$ continuation of $\varkappa^{(n+1)}(\lambda, \varphi)$ to the complex $\frac{1}{2} k^{-r_{n}^{\prime}-\delta}$-neighborhood of $\omega^{(n+1)}(k, \delta, \tau)$ such that $\lambda^{(n+1)}\left(\vec{\varkappa}^{(n+1)}(\lambda, \varphi)\right)=\lambda$. Function $\varkappa^{(n+1)}(\lambda, \varphi)$ can be represented as $\varkappa^{(n+1)}(\lambda, \varphi)=\varkappa^{(n)}(\lambda, \varphi)+h^{(n+1)}(\lambda, \varphi)$, where

$$
\begin{aligned}
& \left|h^{(n+1)}(\varphi)\right|=O_{1}\left(k^{-\frac{1}{5} \beta k^{r_{n}-1^{-r} n-2}-1}\right), \\
& \frac{\partial h^{(n+1)}}{\partial \varphi}=O_{2}\left(k^{-\frac{1}{5} \beta k^{r_{n}-1}-r_{n-2}-1+r_{n}^{\prime}+\delta}\right)
\end{aligned}
$$

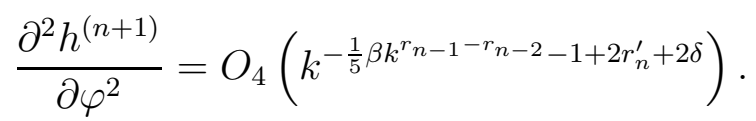

Let us consider the set of points in $\mathbb{R}^{2}$ given by the formula: $\vec{\varkappa}=\vec{\varkappa}^{(n+1)}(\varphi), \quad \varphi \in$ $\omega^{(n+1)}(k, \delta, \tau)$. By Lemma 7.21 this set of points is a slight distortion of $\mathcal{D}_{n}$. All the points of this curve satisfy the equation $\lambda^{(n+1)}\left(\vec{\varkappa}^{(n+1)}(\varphi)\right)=k^{2}$. We call it isoenergetic surface of the operator $H^{(n+1)}$ and denote by $\mathcal{D}_{n+1}$.

\section{Isoenergetic Sets. Generalized Eigenfunctions of $H$}

\subsection{Construction of Limit-Isoenergetic Set}

At every step $n$ we constructed a set $\mathcal{B}_{n}(\lambda), \mathcal{B}_{n}(\lambda) \subset \mathcal{B}_{n-1}(\lambda) \subset S_{1}$, and a function $\varkappa^{(n)}(\lambda, \vec{\nu}), \vec{\nu} \in \mathcal{B}_{n}(\lambda)$, with the following properties. The set $\mathcal{D}_{n}(\lambda)$ of vectors $\vec{\varkappa}=$ $\varkappa^{(n)}(\lambda, \vec{\nu}) \vec{\nu}, \vec{\nu} \in \mathcal{B}_{n}(\lambda)$, is a slightly distorted circle with holes, see formula (19) and Lemmas [3.11, 4.4, 5.5, 6.6, 7.21. For any $\vec{\varkappa}^{(n)}(\lambda, \vec{\nu}) \in \mathcal{D}_{n}(\lambda)$ there is a single eigenvalue of $H^{(n)}\left(\vec{\varkappa}^{(n)}\right)$ equal to $\lambda$ and given by the perturbation series. Let $\mathcal{B}_{\infty}(\lambda)=\bigcap_{n=1}^{\infty} \mathcal{B}_{n}(\lambda)$. Since $\mathcal{B}_{n+1} \subset \mathcal{B}_{n}$ for every $n, \mathcal{B}_{\infty}(\lambda)$ is a unit circle with infinite number of holes, more and more holes of smaller and smaller size appearing at each step.

Lemma 8.1. The length of $\mathcal{B}_{\infty}(\lambda)$ satisfies estimate (8) with $\gamma_{4}=37 \mu \delta$.

Proof. Using Lemmas 3.1 (part 3), 3.32, 4.29, 5.24 and 7.15 and considering that $r_{n}>>37 \delta \mu$, we easily conclude that $L\left(\mathcal{B}_{n}\right)=\left(2 \pi+O\left(k^{-37 \mu \delta}\right)\right), k=\lambda^{1 / 2}$ uniformly in

$n$. Since $\mathcal{B}_{n}$ is a decreasing sequence of sets, (8) holds.

Let us consider $\varkappa_{\infty}(\lambda, \vec{\nu})=\lim _{n \rightarrow \infty} \varkappa^{(n)}(\lambda, \vec{\nu}), \quad \vec{\nu} \in \mathcal{B}_{\infty}(\lambda)$. 
Lemma 8.2. The limit $\varkappa_{\infty}(\lambda, \vec{\nu})$ exists for any $\vec{\nu} \in \mathcal{B}_{\infty}(\lambda)$ and the following estimates hold10:

$$
\begin{aligned}
& \left|\varkappa_{\infty}(\lambda, \vec{\nu})-\lambda^{1 / 2}\right|<c k^{-3+(80 \mu+6) \delta}, \\
& \left|\varkappa_{\infty}(\lambda, \vec{\nu})-\varkappa^{(1)}(\lambda, \vec{\nu})\right|<c k^{-k^{\delta}(2 Q)^{-1}} k^{-1}, \\
& \left|\varkappa_{\infty}(\lambda, \vec{\nu})-\varkappa^{(n)}(\lambda, \vec{\nu})\right|<k^{-\frac{1}{5} \beta k^{r_{n-1}-r_{n-2}}}, \quad n \geq 2, \quad r_{0}:=\delta_{*} .
\end{aligned}
$$

Corollary 8.3. For every $\vec{\nu} \in \mathcal{B}_{\infty}(\lambda)$ estimate (9) holds, where $\gamma_{5}=(3-(80 \mu+6) \delta) / 2>0$.

The lemma easily follows from the estimates (68), (203), (325), (382) and (428).

Estimates (69), (204) (326), (383) and (429) justify convergence of the series $\sum_{n=1}^{\infty} \frac{\partial h_{n}}{\partial \varphi}$, and hence, of the sequence $\frac{\partial \varkappa^{(n)}}{\partial \varphi}$. We denote the limit of this sequence by $\frac{\partial \varkappa_{\infty}}{\partial \varphi}$.

Lemma 8.4. The estimate (23) with $\gamma_{11}=(3-(120 \mu+7) \delta) / 2>0$, holds for any $\vec{\nu} \in \mathcal{B}_{\infty}(\lambda)$.

We define $\mathcal{D}_{\infty}(\lambda)$ by (7). Clearly, $\mathcal{D}_{\infty}(\lambda)$ is a slightly distorted circle of radius $k$ with infinite number of holes. We can assign a tangent vector $\frac{\partial \varkappa_{\infty}}{\partial \varphi} \vec{\nu}+\varkappa_{\infty} \vec{\mu}, \vec{\mu}=(-\sin \varphi, \cos \varphi)$ to the curve $\mathcal{D}_{\infty}(\lambda)$, this tangent vector being the limit of corresponding tangent vectors for curves $\mathcal{D}_{n}(\lambda)$ at points $\vec{\varkappa}^{(n)}(\lambda, \vec{\nu})$ as $n \rightarrow \infty$.

Next we show that $\mathcal{D}_{\infty}(\lambda)$ is an isoenergetic curve for $H$. Namely for every $\vec{\varkappa} \in \mathcal{D}_{\infty}(\lambda)$ there is a generalized eigenfunction $\Psi_{\infty}(\vec{\varkappa}, \vec{x})$ such that $H \Psi_{\infty}=\lambda \Psi_{\infty}$.

\subsection{Generalized Eigenfunctions of $H$}

We show that for every $\vec{\varkappa}$ in a set

$$
\mathcal{G}_{\infty}=\cup_{\lambda>\lambda_{*}} \mathcal{D}_{\infty}(\lambda), \quad \lambda_{*}=k_{*}^{2},
$$

there is a solution $\Psi_{\infty}(\vec{\varkappa}, \vec{x})$ of the equation for eigenfunctions:

$$
-\Delta \Psi_{\infty}(\vec{\varkappa}, \vec{x})+V(\vec{x}) \Psi_{\infty}(\vec{\varkappa}, \vec{x})=\lambda_{\infty}(\vec{\varkappa}) \Psi_{\infty}(\vec{\varkappa}, \vec{x}),
$$

which can be represented in the form

$$
\left.\Psi_{\infty}(\vec{\varkappa}, \vec{x})=e^{i\langle\vec{\varkappa}, \vec{x}\rangle}\left(1+u_{\infty}(\vec{\varkappa}, \vec{x})\right), \quad \| u_{\infty}(\vec{\varkappa}, \vec{x})\right) \|_{L_{\infty}\left(\mathbb{R}^{2}\right)}=O\left(|\vec{\varkappa}|^{-\gamma_{1}}\right),
$$

\footnotetext{
${ }^{10}$ Here and below to make the formulations shorter we will use the notation $r_{0}$ which may be equal either $\delta$ or $\delta_{*}$. In any case $\Omega\left(r_{0}\right):=\Omega(\delta)$. In all other situations where $r_{0}$ appears we will specify what it is equal to.
} 
where $u_{\infty}(\vec{\varkappa}, \vec{x})$ is a quasi-periodic function, $\gamma_{1}=1-45 \mu \delta>0$; the eigenvalue $\lambda_{\infty}(\vec{\varkappa})$ satisfies the asymptotic formula:

$$
\lambda_{\infty}(\vec{\varkappa})=|\vec{\varkappa}|^{2}+O\left(|\vec{\varkappa}|^{-\gamma_{2}}\right), \quad \gamma_{2}=2-(80 \mu+6) \delta>0 .
$$

We also show that the set $\mathcal{G}_{\infty}$ satisfies (무).

In fact, by (431), any $\vec{\varkappa} \in \mathcal{D}_{\infty}(\lambda)$ belongs to the $k^{-\frac{1}{5} \beta k^{r_{n}-1}-r_{n-2}}$-neighborhood of $\mathcal{D}_{n}(\lambda), \quad n \geq 3$. Let us consider spectral projectors $\mathcal{E}^{(n)}$, each of them being defined in a finite dimensional space of sequences with indices in $\Omega\left(r_{n-1}\right)$. We extend each of them to the whole space $\ell^{2}\left(\mathbb{Z}^{4}\right)$ by putting it to be zero into the orthogonal complement of $\ell^{2}\left(\Omega\left(r_{n-1}\right)\right)$. This way they all act in space $\ell^{2}\left(\mathbb{Z}^{4}\right)$. Applying the perturbation formulae proved in the previous sections (see Corollaries [3.4, 4.2, 5.2, 6.2, 7.18), we obtain the following inequalities:

$$
\begin{aligned}
& \left\|\mathcal{E}^{(1)}(\vec{\varkappa})-\mathcal{E}_{0}(\vec{\varkappa})\right\|_{1}<c k^{-\gamma_{0}}, \quad \gamma_{0}:=1-44 \mu \delta, \\
& \left\|\mathcal{E}^{(2)}(\vec{\varkappa})-\mathcal{E}^{(1)}(\vec{\varkappa})\right\|_{1}<k^{-k^{\delta}(4 Q)^{-1}} \text {, } \\
& \left\|\mathcal{E}^{(n)}(\vec{\varkappa})-\mathcal{E}^{(n-1)}(\vec{\varkappa})\right\|_{1}<2 k^{-\frac{1}{10} \beta k^{r_{n-2}-r_{n-3}}}, \quad n \geq 3, \quad r_{0}:=\delta_{*}, \\
& \left.\left|\lambda^{(1)}(\vec{\varkappa})-\right| \vec{\varkappa}\right|^{2}\left|<c k^{-\gamma_{2}}, \quad\right| \lambda^{(2)}(\vec{\varkappa})-\lambda^{(1)}(\vec{\varkappa}) \mid<c k^{-k^{\delta}(2 Q)^{-1}}, \\
& \left|\lambda^{(n)}(\vec{\varkappa})-\lambda^{(n-1)}(\vec{\varkappa})\right|<2 k^{-\frac{1}{5} \beta k^{r_{n-2}-r_{n-3}}}, \quad n \geq 3, \quad r_{0}:=\delta_{*},
\end{aligned}
$$

where $\lambda^{(n+1)}(\vec{\varkappa})$ is the eigenvalue corresponding to $\mathcal{E}^{(n+1)}(\vec{\varkappa}), \mathcal{E}_{0}(\vec{\varkappa})$ corresponds to $V=0$.

Remark 8.5. We see from 4 431), that any $\vec{\varkappa} \in \mathcal{D}_{\infty}(\lambda)$ belongs to the $k^{-\frac{1}{5} \beta k^{r_{n}-1}-r_{n-2}}$ neighborhood of $\mathcal{D}_{n}(\lambda), n \geq 3$. Applying perturbation formulae for $n$-th step, we easily obtain that there is an eigenvalue $\lambda^{(n)}(\vec{\varkappa})$ of $H^{(n)}(\vec{\varkappa})$ satisfying the estimate $\lambda^{(n)}(\vec{\varkappa})=$ $\lambda+\delta_{n}, \delta_{n}=o(1)$ as $n \rightarrow \infty$, the eigenvalue $\lambda^{(n)}(\vec{\varkappa})$ being given by a perturbation series of the type 413). Hence, for every $\vec{\varkappa} \in \mathcal{D}_{\infty}(\lambda)$ there is a limit:

$$
\lim _{n \rightarrow \infty} \lambda^{(n)}(\vec{\varkappa})=\lambda \text {. }
$$

Let $\mathbf{v}^{(n)}$ be a unit vector corresponding to the projection $\mathcal{E}^{(n)}(\vec{\varkappa}), \mathcal{E}^{(n)}(\vec{\varkappa})=\left(\cdot, \mathbf{v}^{(n)}\right) \mathbf{v}^{(n)}$, $\mathbf{v}^{(n)}=\left\{v_{\mathbf{s}}^{(n)}\right\}_{\mathbf{s} \in \mathbb{Z}^{4}} \in \ell^{2}\left(\mathbb{Z}^{4}\right)$. By construction, $v_{\mathbf{s}}^{(n)}=0$ when $\mathbf{s} \notin \Omega\left(r_{n-1}\right)$. Let us consider the linear combination of exponents corresponding to this vector:

$$
\Psi_{n}(\vec{\varkappa}, \vec{x})=\sum_{\mathbf{s} \in \Omega\left(r_{n-1}\right)} v_{\mathbf{s}}^{(n)} e^{i\left\langle\vec{\varkappa}+\vec{p}_{\mathbf{s}}, \vec{x}\right\rangle} .
$$

Lemma 8.6. Function $\Psi_{n}(\vec{\varkappa}, \vec{x}), n \geq 4$, satisfies the equation:

$$
-\Delta \Psi_{n}(\vec{\varkappa}, \vec{x})+V(\vec{x}) \Psi_{n}(\vec{\varkappa}, \vec{x})=\lambda_{n}(\vec{\varkappa}) \Psi_{n}(\vec{\varkappa}, \vec{x})+g_{n}(\vec{\varkappa}, \vec{x}),
$$


the vector $\mathbf{g}_{n}$ of the Fourier coefficients of $g_{n}(\vec{\varkappa}, \vec{x})$ satisfying the estimate:

$$
\left\|\mathbf{g}_{n}\right\|_{\ell^{1}\left(\mathbb{Z}^{4}\right)}<k^{-k^{\frac{1}{2} r_{n-1}}} .
$$

Coefficients $\left(\mathbf{g}_{n}\right)_{\mathbf{s}}$ can differ from zero only when $k^{r_{n-1}}<\left\|\left|\vec{p}_{\mathbf{s}} \|\right| \leq k^{r_{n-1}}+\right.$ Q. Function $g_{n}(\vec{\varkappa}, \vec{x})$ obeys the estimate:

$$
\left\|g_{n}\right\|_{L_{\infty}\left(\mathbb{R}^{2}\right)}<k^{-k^{\frac{1}{2} r_{n}-1}} .
$$

Proof. Let $P\left(r_{n-1}\right)$ be the projection in $\ell^{2}\left(\mathbb{Z}^{4}\right)$ on the subspace corresponding to $\Omega\left(r_{n-1}\right)$. By construction, $P\left(r_{n-1}\right) \mathbf{v}^{(n)}=\mathbf{v}^{(n)}$ and

$$
H_{0} \mathbf{v}^{(n)}+P\left(r_{n-1}\right) V P\left(r_{n-1}\right) \mathbf{v}^{(n)}=\lambda_{n}(\vec{\varkappa}) \mathbf{v}^{(n)} .
$$

Since $V$ is a trigonometric polynomial,

$$
\left(I-P\left(r_{n-1}\right)\right) V P\left(r_{n-1}\right)=\left(I-P\left(r_{n-1}\right)\right) V P_{\partial}\left(r_{n-1}\right),
$$

where $P_{\partial}\left(r_{n-1}\right)_{\mathbf{m m}}=1$ only if $\mathbf{m}$ is in the $Q$-vicinity of the boundary. Using (420) with $n$ instead of $n+1$, we obtain: $\left\|P_{\partial}\left(r_{n-1}\right) \mathcal{E}^{(n)}\right\|<k^{-k^{r_{n-1}(1-\gamma)}}$ and, hence, $\left\|P_{\partial}\left(r_{n-1}\right) \mathbf{v}^{(n)}\right\|<$ $k^{-k^{r_{n-1}(1-\gamma)}}$. Therefore, $\left\|\left(I-P\left(r_{n-1}\right)\right) V P\left(r_{n-1}\right) \mathbf{v}^{(n)}\right\|<\|V\| k^{-k^{r_{n-1}(1-\gamma)}}$. It follows that $H(\vec{\varkappa}) \mathbf{v}^{(n)}=\lambda_{n}(\vec{\varkappa}) \mathbf{v}^{(n)}+\mathbf{g}_{n}$, where $\left\|\mathbf{g}_{n}\right\|_{\ell^{2}\left(\mathbb{Z}^{4}\right)}<\|V\| k^{-k^{r_{n-1}(1-\gamma)}}$. Note that elements $\left(\mathbf{g}_{n}\right)_{\mathbf{s}}$ are equal to zero when $\left\|\left|\vec{p}_{\mathbf{s}} \|\right| \leq k^{r_{n-1}}\right.$ or $\|\left|\vec{p}_{\mathbf{s}} \|\right|>k^{r_{n-1}}+Q$. Therefore, (438) holds. Estimate (439) follows.

Lemma 8.7. Functions $\Psi_{n}(\vec{\varkappa}, \vec{x})$ satisfy the inequalities:

$$
\begin{gathered}
\left\|\Psi_{1}-\Psi_{0}\right\|_{L_{\infty}\left(\mathbb{R}^{2}\right)}<c k^{-\gamma_{0}+2 \delta}, \quad\left\|\Delta\left(\Psi_{1}-\Psi_{0}\right)\right\|_{L_{\infty}\left(\mathbb{R}^{2}\right)}<c k^{-\gamma_{0}+2 \delta+2}, \quad \Psi_{0}(\vec{x})=e^{i\langle\vec{x}, \vec{x}\rangle}, \\
\left\|\Psi_{2}-\Psi_{1}\right\|_{L_{\infty}\left(\mathbb{R}^{2}\right)}<c k^{-k^{\delta}(4 Q)^{-1}+2 r_{1}} \\
\left\|\Delta\left(\Psi_{2}-\Psi_{1}\right)\right\|_{L_{\infty}\left(\mathbb{R}^{2}\right)}<c k^{-k^{\delta}(4 Q)^{-1}+4 r_{1}} \\
\left\|\Psi_{n}-\Psi_{n-1}\right\|_{L_{\infty}\left(\mathbb{R}^{2}\right)}<c k^{-\frac{1}{10} \beta k^{r_{n-2}-r_{n-3}+2 r_{n-1}}} \\
\left\|\Delta\left(\Psi_{n}-\Psi_{n-1}\right)\right\|_{L_{\infty}\left(\mathbb{R}^{2}\right)}<c k^{-\frac{1}{10} \beta k^{r_{n-2}-r_{n-3}+4 r_{n-1}}, \quad n \geq 3, \quad r_{0}:=\delta_{*} .}
\end{gathered}
$$

Corollary 8.8. All functions $\Psi_{n}, n=0,1, \ldots$, obey the estimate $\left\|\Psi_{n}\right\|_{L_{\infty}\left(\mathbb{R}^{2}\right)}<1+$ $C k^{-\gamma_{0}+2 \delta}$ uniformly in $n$.

Proof. Using (435) and considering that $v_{\mathbf{s}}^{(n)}$ are equal to zero when $\left\|\left|\vec{p}_{\mathbf{s}} \|\right|>k^{r_{n-1}}\right.$, we obtain

$$
\begin{gathered}
\left\|\mathbf{v}^{(1)}-\mathbf{v}^{(0)}\right\|_{\ell^{1}\left(\mathbb{Z}^{4}\right)}<c k^{-\gamma_{0}+2 \delta}, \quad\left\|\mathbf{v}^{(2)}-\mathbf{v}^{(1)}\right\|_{\ell^{1}\left(\mathbb{Z}^{4}\right)}<c k^{-k^{\delta}(4 Q)^{-1}+2 r_{1}}, \\
\left\|\mathbf{v}^{(n)}-\mathbf{v}^{(n-1)}\right\|_{\ell^{1}\left(\mathbb{Z}^{4}\right)}<c k^{-\frac{1}{10} \beta k^{r}-_{-2}-r_{n-3}+2 r_{n-1}}, \quad n \geq 3 . \\
\left\|H_{0}\left(\mathbf{v}^{(1)}-\mathbf{v}^{(0)}\right)\right\|_{\ell^{1}\left(\mathbb{Z}^{4}\right)}<c k^{-\gamma_{0}+2 \delta+2}, \quad\left\|H_{0}\left(\mathbf{v}^{(2)}-\mathbf{v}^{(1)}\right)\right\|_{\ell^{1}\left(\mathbb{Z}^{4}\right)}<c k^{-k^{\delta}(4 Q)^{-1}+4 r_{1}}, \\
\left\|H_{0}\left(\mathbf{v}^{(n)}-\mathbf{v}^{(n-1)}\right)\right\|_{\ell^{1}\left(\mathbb{Z}^{4}\right)}<c k^{-\frac{1}{10} \beta k^{r_{n-2}-r_{n-3}+4 r_{n-1}}, \quad n \geq 3, \quad r_{0}:=\delta_{*} .}
\end{gathered}
$$

Now (440) - (442) easily follow. 
Theorem 8.9. For every $\lambda>k_{*}^{2}$ and $\vec{\varkappa} \in \mathcal{D}_{\infty}(\lambda)$ the sequence of functions $\Psi_{n}(\vec{\varkappa}, \vec{x})$ converges in $L_{\infty}\left(\mathbb{R}^{2}\right)$ and $W_{2, l o c}^{2}\left(\mathbb{R}^{2}\right)$. The limit function $\Psi_{\infty}(\vec{\varkappa}, \vec{x}):=\lim _{n \rightarrow \infty} \Psi_{n}(\vec{\varkappa}, \vec{x})$, is a quasi-periodic function:

$$
\Psi_{\infty}(\vec{\varkappa}, \vec{x})=\sum_{\mathbf{s} \in \mathbb{Z}^{4}}\left(v_{\infty}\right)_{\mathbf{s}} e^{i\left\langle\vec{\varkappa}+\vec{p}_{\mathbf{s}}, \vec{x}\right\rangle},
$$

where $\mathbf{v}_{\infty}=\left\{\left(v_{\infty}\right)_{\mathbf{s}}\right\}_{\mathbf{s} \in \mathbb{Z}^{4}} \in \ell^{1}\left(\mathbb{Z}^{4}\right)$ and $\left\|\mathbf{v}_{\infty}\right\|_{\ell^{2}\left(\mathbb{Z}^{4}\right)}=1$. The function $\Psi_{\infty}(\vec{\varkappa}, \vec{x})$ satisfies the equation

$$
-\Delta \Psi_{\infty}(\vec{\varkappa}, \vec{x})+V(\vec{x}) \Psi_{\infty}(\vec{\varkappa}, \vec{x})=\lambda \Psi_{\infty}(\vec{\varkappa}, \vec{x}) .
$$

It can be represented in the form

$$
\Psi_{\infty}(\vec{\varkappa}, \vec{x})=e^{i\langle\vec{\varkappa}, \vec{x}\rangle}\left(1+u_{\infty}(\vec{\varkappa}, \vec{x})\right),
$$

where $u_{\infty}(\vec{\varkappa}, \vec{x})$ is a quasi-periodic function:

$$
\begin{gathered}
u_{\infty}(\vec{\varkappa}, \vec{x})=\sum_{n=1}^{\infty} u_{n}(\vec{\varkappa}, \vec{x}), \\
u_{n}(\vec{\varkappa}, \vec{x})=\sum_{\mathbf{s} \in \Omega\left(r_{n-1}\right)}\left(v_{\mathbf{s}}^{(n)}-v_{\mathbf{s}}^{(n-1)}\right) e^{i\left\langle\vec{p}_{\mathbf{s}}, \vec{x}\right\rangle},
\end{gathered}
$$

functions $u_{n}$ satisfying the estimates:

$$
\begin{aligned}
& \left\|u_{1}\right\|_{L_{\infty}\left(\mathbb{R}^{2}\right)}<c k^{-\gamma_{0}+2 \delta}, \quad\left\|u_{2}\right\|_{L_{\infty}\left(\mathbb{R}^{2}\right)}<c k^{-k^{\delta}(4 Q)^{-1}+2 r_{1}},
\end{aligned}
$$

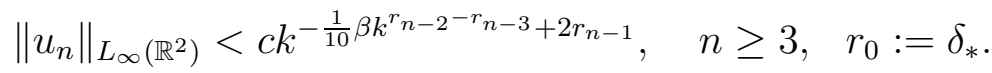

Corollary 8.10. Function $u_{\infty}(\vec{\varkappa}, \vec{x})$ obeys the estimate (433).

Proof. Using (443), (444), we obtain that the sequence $\mathbf{v}^{(n)}$ has the limit in $\ell^{1}\left(\mathbb{Z}^{4}\right)$. We denote this limit by $\mathbf{v}_{\infty}$. Since, vectors $\mathbf{v}^{(n)}$ are normalized in $\ell^{2}\left(\mathbb{Z}^{4}\right)$,

$$
\left\|\mathbf{v}_{\infty}\right\|_{\ell^{2}\left(\mathbb{Z}^{4}\right)}=1 \text {. }
$$

By (442), we obtain that $\Psi_{n}(\vec{\varkappa}, \vec{x})$ is a Cauchy sequence in $L_{\infty}\left(\mathbb{R}^{2}\right)$ and $W_{2, l o c}^{2}\left(\mathbb{R}^{2}\right)$. Let $\Psi_{\infty}(\vec{\varkappa}, \vec{x})=\lim _{n \rightarrow \infty} \Psi_{n}(\vec{\varkappa}, \vec{x})$. This limit is defined pointwise uniformly in $\vec{x}$ and in $W_{2, l o c}^{2}\left(\mathbb{R}^{2}\right)$. Noting also that $\lim \lambda_{n}(\vec{\varkappa})=\lambda$, and taking into account Lemma 8.6 we obtain that (446) holds.

Let us show that $\Psi_{\infty}$ is a quasi-periodic function. Obviously,

$$
\Psi_{\infty}=\Psi_{0}+\sum_{n=1}^{\infty}\left(\Psi_{n}-\Psi_{n-1}\right),
$$

the series converging in $L_{\infty}\left(\mathbb{R}^{2}\right)$ by (442). Introducing $u_{n}:=e^{-i\langle\vec{x}, \vec{x}\rangle}\left(\Psi_{n}-\Psi_{n-1}\right)$, we arrive at (447), (448). Note that $u_{n}$ has a form (449) Estimates (450), (451) follow from (440), (442). 
Theorem 8.11. Formulae (432), 433) and (434) hold for every $\vec{\varkappa} \in \mathcal{G}_{\infty}$. The set $\mathcal{G}_{\infty}$ is Lebesgue measurable and satisfies (6) with $\gamma_{3}=37 \mu \delta$.

Proof. By Theorem 8.9, (432), (433) hold, where $\lambda_{\infty}(\vec{\varkappa})=\lambda$ for $\vec{\varkappa} \in \mathcal{D}_{\infty}(\lambda)$. Using (91), which is proven in Corollary 8.3, with $\varkappa_{\infty}=|\vec{\varkappa}|$, we easily obtain (434). It remains to prove (6). Let us consider a small region $U_{n}\left(\lambda_{0}\right)$ around an isoenergetic surface $\mathcal{D}_{n}\left(\lambda_{0}\right), \lambda_{0}>k_{*}^{2}$. Namely, $U_{n}\left(\lambda_{0}\right)=\cup_{\left|\lambda-\lambda_{0}\right|<\varepsilon_{0}^{(n+1)}} \mathcal{D}_{n}(\lambda), k=\lambda_{0}^{1 / 2}$. By Theorem 7.17 the construction of the $n$-th non-resonant set is stable in $\varepsilon_{0}^{(n)}$-neighborhood of $\lambda_{0}$. Therefore, in fact, we can (and for the sake of convenience will) assume that the sets $\omega^{(n)}(\lambda)$ are chosen to be equal to $\omega^{(n)}\left(\lambda_{0}\right)$ for $\left|\lambda-\lambda_{0}\right|<\varepsilon_{0}^{(n)}$. Thus, $U_{n}\left(\lambda_{0}\right)$ is an open set (a distorted ring with holes) and $\left|U_{n}\left(\lambda_{0}\right)\right|=\varepsilon_{0}^{(n)} 2 \pi\left(1+O\left(k^{-37 \mu \delta}\right)\right)$. It easily follows from (70) and (428) that $U_{n+1} \subset U_{n}$. Definition of $\mathcal{D}_{\infty}\left(\lambda_{0}\right)$ yield: $\mathcal{D}_{\infty}\left(\lambda_{0}\right)=\cap_{n=1}^{\infty} U_{n}\left(\lambda_{0}\right)$. Hence, $\mathcal{G}_{\infty}=\cap_{n=1}^{\infty} \mathcal{G}_{n}$, where

$$
\mathcal{G}_{n}=\cup_{\lambda>\lambda_{*}} \mathcal{D}_{n}(\lambda), \quad \lambda_{*}=k_{*}^{2} .
$$

Considering that $U_{n+1} \subset U_{n}$ for every $\lambda_{0}>\lambda_{*}$, we obtain $\mathcal{G}_{n+1} \subset \mathcal{G}_{n}$. Hence, $\left|\mathcal{G}_{\infty} \cap \mathbf{B}_{\mathbf{R}}\right|=$ $\lim _{n \rightarrow \infty}\left|\mathcal{G}_{n} \cap \mathbf{B}_{\mathbf{R}}\right|$. Calculating the volume of the region $\cup_{\lambda_{*}<\lambda<R^{2}} U_{n}(\lambda)$, we easily conclude $\left|\mathcal{G}_{n} \cap \mathbf{B}_{\mathbf{R}}\right|=\left|\mathbf{B}_{\mathbf{R}}\right|\left(1+O\left(R^{-37 \mu \delta}\right)\right)$ uniformly in $n$. Thus, we have obtained (6) with $\gamma_{3}=37 \mu \delta$.

Theorem 8.12 (Bethe-Sommerfeld Conjecture). The spectrum of operator $H$ contains a semi-axis.

Proof. The theorem immediately follows from the fact that the equation (446) has a bounded solution $\Psi_{\infty}(\vec{\varkappa}, \vec{x})$ for every sufficiently large $\lambda$.

\section{Proof of Absolute Continuity of the Spectrum}

The proof is somewhat analogous to that for the case of limit-periodic potentials [31]. We will just refer to [31] in some places. We also note that proofs of absolutely continuous spectrum through establishing localization in the momentum space have been done for 1D operators in the past (see [41], [42]).

\subsection{Operators $E_{n}\left(\mathcal{G}_{n}^{\prime}\right), \mathcal{G}_{n}^{\prime} \subset \mathcal{G}_{n}$}

Let us consider the open sets $\mathcal{G}_{n}$ given by (453). There is a family of eigenfunctions $\Psi_{n}(\vec{\varkappa}, \vec{x}), \vec{\varkappa} \in \mathcal{G}_{n}$, of the operator $H^{(n)}$, which are described by the perturbation formulas (11), (15). Let, $\mathcal{G}_{n}^{\prime} \subset \mathcal{G}_{n}$, where $\mathcal{G}_{n}^{\prime}$ is Lebesgue measurable and bounded. Let

$$
E_{n}\left(\mathcal{G}_{n}^{\prime}\right) F=\frac{1}{4 \pi^{2}} \int_{\mathcal{G}_{n}^{\prime}}\left(F, \Psi_{n}(\vec{\varkappa})\right) \Psi_{n}(\vec{\varkappa}) d \vec{\varkappa}
$$


for any $F \in C_{0}^{\infty}\left(\mathbb{R}^{2}\right)$, here and below $(\cdot, \cdot)$ is the canonical scalar product in $L_{2}\left(\mathbb{R}^{2}\right)$, i.e.,

$$
\left(F, \Psi_{n}(\vec{\varkappa})\right)=\int_{\mathbb{R}^{2}} F(\vec{x}) \overline{\Psi_{n}(\vec{\varkappa}, \vec{x})} d \vec{x}
$$

We will show that $E_{n}\left(\mathcal{G}_{n}^{\prime}\right)$ is almost a projector in $L_{2}\left(\mathbb{R}^{2}\right)$ in the sense: $E_{n}\left(\mathcal{G}_{n}^{\prime}\right)=E_{n}^{*}\left(\mathcal{G}_{n}^{\prime}\right)$, $E_{n}^{2}\left(\mathcal{G}_{n}^{\prime}\right)=E_{n}\left(\mathcal{G}_{n}^{\prime}\right)+o(1)$, where $o(1)$ is in the class of bounded operators as $n \rightarrow \infty$. First, we note that (454) can be rewritten in the form:

$$
\begin{gathered}
E_{n}\left(\mathcal{G}_{n}^{\prime}\right)=S_{n}\left(\mathcal{G}_{n}^{\prime}\right) T_{n}\left(\mathcal{G}_{n}^{\prime}\right), \\
T_{n}: L_{2}\left(\mathbb{R}^{2}\right) \rightarrow L_{2}\left(\mathcal{G}_{n}^{\prime}\right), \quad S_{n}: L_{2}\left(\mathcal{G}_{n}^{\prime}\right) \rightarrow L_{2}\left(\mathbb{R}^{2}\right), \\
T_{n} F=\frac{1}{2 \pi}\left(F, \Psi_{n}(\vec{\varkappa})\right) \text { for any } F \in C_{0}^{\infty}\left(\mathbb{R}^{2}\right),
\end{gathered}
$$

$T_{n} F$ being in $L_{\infty}\left(\mathcal{G}_{n}^{\prime}\right)$, and,

$$
S_{n} f=\frac{1}{2 \pi} \int_{\mathcal{G}_{n}^{\prime}} f(\vec{\varkappa}) \Psi_{n}(\vec{\varkappa}, \vec{x}) d \vec{\varkappa} \text { for any } f \in L_{\infty}\left(\mathcal{G}_{n}^{\prime}\right) .
$$

Note that $S_{n} f \in L_{2}\left(\mathbb{R}^{2}\right)$, since $\Psi_{n}$ is a finite combination of exponentials $e^{i\left\langle\vec{\varkappa}+\vec{p}_{\mathbf{q}}, \vec{x}\right\rangle}$.

Lemma 9.1. Let $\mathcal{G}_{n}^{\prime}$ be bounded and $f(\vec{\varkappa}), g(\vec{\varkappa}) \in L_{\infty}\left(\mathcal{G}_{n}^{\prime}\right)$. Then,

$$
\left(S_{n} f, S_{n} g\right)_{L_{2}\left(\mathbb{R}^{2}\right)}={ }_{n \rightarrow \infty}(f, g)_{L_{2}\left(\mathcal{G}_{n}^{\prime}\right)}+o(1)\|f\|_{L_{2}\left(\mathcal{G}_{n}^{\prime}\right)}\|g\|_{L^{2}\left(\mathcal{G}_{n}^{\prime}\right)}
$$

where o(1) goes to zero uniformly in $f, g$ and $\mathcal{G}_{n}^{\prime}$ as $n \rightarrow \infty$; namely, $|o(1)|<\xi_{*}^{-r_{n-3}\left(\xi_{*}\right)}$, where $\xi_{*}=\inf _{\vec{\xi} \in \mathcal{G}_{n}^{\prime}}|\vec{\xi}|$.

Corollary 9.2. The following relation holds:

$$
\left|\left(S_{n} f, S_{n} g\right)_{L_{2}\left(\mathbb{R}^{2}\right)}\right|<(1+o(1))\|f\|_{L_{\infty}\left(\mathcal{G}_{n}^{\prime}\right)}\|g\|_{L_{\infty}\left(\mathcal{G}_{n}^{\prime}\right)}\left|\mathcal{G}_{n}^{\prime}\right|,
$$

where $\left|\mathcal{G}_{n}^{\prime}\right|$ is the Lebesgue measure of $\mathcal{G}_{n}^{\prime}$.

Corollary 9.3. The operator $S_{n}$ is bounded and $\left\|S_{n}\right\|={ }_{n \rightarrow \infty} 1+o(1)$.

Proof. The function $\Psi_{n}(\vec{\varkappa}, \vec{x})$ can be represented as a combination of plane waves:

$$
\Psi_{n}(\vec{\varkappa}, \vec{x})=\sum_{\mathbf{m} \in \mathbb{Z}^{4}} v_{\mathbf{m}}^{(n)}(\vec{\varkappa}) \exp \left\{i\left\langle\vec{\varkappa}+\vec{p}_{\mathbf{m}}, \vec{x}\right\rangle\right\}
$$

where $v_{\mathbf{m}}^{(n)}(\vec{\varkappa})$ are Fourier coefficients. By construction, $v_{\mathbf{m}}^{(n)}(\vec{\varkappa})=0$, when $\mathbf{m} \notin \Omega\left(r_{n-1}\right)$. Let $\mathbf{v}^{(n)}(\vec{\varkappa})$ be the vector in $\ell^{2}\left(\mathbb{Z}^{2}\right)$ with components equal to $v_{\mathbf{m}}^{(n)}(\vec{\varkappa})$. Note that the size 
of $\Omega\left(r_{n-1}\right)$ depend on $\varkappa=|\vec{\varkappa}|$; to stress this fact we will use here the notations $\Omega\left(r_{n-1}, \varkappa\right)$ and $r_{n-1}(\varkappa)$. The Fourier transform $\widehat{\Psi}_{n}$ is a combination of $\delta$-functions:

$$
\widehat{\Psi}_{n}(\vec{\varkappa}, \vec{\xi})=2 \pi \sum_{\mathbf{m} \in \mathbb{Z}^{4}} v_{\mathbf{m}}^{(n)}(\vec{\varkappa}) \delta\left(\vec{\xi}+\vec{\varkappa}+\vec{p}_{\mathbf{m}}\right)
$$

From this, we easily compute the Fourier transform of $\left(S_{n} f\right)(\vec{x})$ :

$$
\left(\widehat{S_{n} f}\right)(\vec{\xi})=\sum_{\mathbf{m} \in \mathbb{Z}^{4}} v_{\mathbf{m}}^{(n)}\left(-\vec{\xi}-\vec{p}_{\mathbf{m}}\right) f\left(-\vec{\xi}-\vec{p}_{\mathbf{m}}\right) \chi\left(\mathcal{G}_{n}^{\prime},-\vec{\xi}-\vec{p}_{\mathbf{m}}\right)
$$

where $\chi\left(\mathcal{G}_{n}^{\prime}, \cdot\right)$ is the characteristic function of $\mathcal{G}_{n}^{\prime}$. Note that $v_{\mathbf{m}}^{(n)}\left(-\vec{\xi}-\vec{p}_{\mathbf{m}}\right) \chi\left(\mathcal{G}_{n}^{\prime},-\vec{\xi}-\vec{p}_{\mathbf{m}}\right)$ can differ from zero only when $\mathbf{m} \in \Omega\left(r_{n-1},\left|\vec{\xi}+\vec{p}_{\mathbf{m}}\right|\right) \subset \Omega\left(r_{n-1}, \xi_{* *}\right), \xi_{* *}=\sup _{\vec{\xi} \in \mathcal{G}_{n}^{\prime}}|\vec{\xi}|$. By Parseval's identity,

$$
\begin{gathered}
\left(S_{n} f, S_{n} g\right)_{L_{2}\left(\mathbb{R}^{2}\right)}=\left(\widehat{S_{n} f}, \widehat{S_{n} g}\right)_{L_{2}\left(\mathbb{R}^{2}\right)}= \\
\int_{\mathbb{R}^{2}} \sum_{\mathbf{m}, \mathbf{m}^{\prime} \in \mathbb{Z}^{4}} T_{\mathbf{m}, \mathbf{m}^{\prime}}(\vec{\xi}) f\left(-\vec{\xi}-\vec{p}_{\mathbf{m}}\right) \bar{g}\left(-\vec{\xi}-\vec{p}_{\mathbf{m}^{\prime}}\right) \chi\left(\mathcal{G}_{n}^{\prime},-\vec{\xi}-\vec{p}_{\mathbf{m}}\right) \chi\left(\mathcal{G}_{n}^{\prime},-\vec{\xi}-\vec{p}_{\mathbf{m}^{\prime}}\right) d \vec{\xi}, \\
T_{\mathbf{m}, \mathbf{m}^{\prime}}(\vec{\xi}):=v_{\mathbf{m}}^{(n)}\left(-\vec{\xi}-\vec{p}_{\mathbf{m}}\right) \overrightarrow{v_{\mathbf{m}^{\prime}}^{(n)}}\left(-\vec{\xi}-\vec{p}_{\mathbf{m}^{\prime}}\right) .
\end{gathered}
$$

Note that, in fact, the summation here is over the finite set $\mathbf{m}, \mathbf{m}^{\prime} \in \Omega\left(r_{n-1}, \xi_{* *}\right)$. Hence we can exchange summation and integration in the above formula. Next, shifting the variable $\vec{\xi}+\vec{p}_{\mathbf{m}} \rightarrow \vec{\xi}$, denoting $\mathbf{m}^{\prime}-\mathbf{m}$ by $\mathbf{m}^{\prime \prime}$ and considering that $\left\langle\mathbf{v}^{(n)}, \mathbf{v}^{(n)}\right\rangle=1$, we obtain:

$$
\begin{gathered}
\left(\widehat{S_{n} f}, \widehat{S_{n} g}\right)_{L_{2}\left(\mathbb{R}^{2}\right)}=(f, g)_{L_{2}\left(\mathcal{G}_{n}^{\prime}\right)}+ \\
\sum_{\mathbf{m}^{\prime \prime} \in \mathbb{Z}^{4} \backslash\{\mathbf{0}\}} \int_{\mathbb{R}^{2}} B_{\mathbf{m}^{\prime \prime}}(\vec{\xi}) f(-\vec{\xi}) \bar{g}\left(-\vec{\xi}-\vec{p}_{\mathbf{m}^{\prime \prime}}\right) \chi\left(\mathcal{G}_{n}^{\prime},-\vec{\xi}\right) \chi\left(\mathcal{G}_{n}^{\prime},-\vec{\xi}-\vec{p}_{\mathbf{m}^{\prime \prime}}\right) d \vec{\xi} \\
B_{\mathbf{m}^{\prime \prime}}(\vec{\xi})=\sum_{\mathbf{m} \in \mathbb{Z}^{4}} v_{\mathbf{m}}^{(n)}(-\vec{\xi}) \widehat{v_{\mathbf{m}+\mathbf{m}^{\prime \prime}}^{(n)}}\left(-\vec{\xi}-\vec{p}_{\mathbf{m}^{\prime \prime}}\right) .
\end{gathered}
$$

Obviously,

$$
B_{\mathbf{m}^{\prime \prime}}=\left\langle\mathbf{v}^{(n)}(-\vec{\xi}), \mathbf{v}_{*}^{(n)}\left(-\vec{\xi}-\vec{p}_{\mathbf{m}^{\prime \prime}}\right)\right\rangle
$$

where $\mathbf{v}_{*}^{(n)}\left(-\vec{\xi}-\vec{p}_{\mathbf{m}^{\prime \prime}}\right)$ the "shifted" eigenvector: $\mathbf{v}_{*}^{(n)}\left(-\vec{\xi}-\vec{p}_{\mathbf{m}^{\prime \prime}}\right):\left(\mathbf{v}_{*}^{(n)}\left(-\vec{\xi}-\vec{p}_{\mathbf{m}^{\prime \prime}}\right)\right)_{\mathbf{m}}=$ $v_{\mathbf{m}+\mathbf{m}^{\prime \prime}}^{(n)}\left(-\vec{\xi}-\vec{p}_{\mathbf{m}^{\prime \prime}}\right)$. To obtain (458), it is enough to prove two estimates for $n \geq 4$ :

$$
\sum_{\left\|\vec{p}_{\mathbf{m}^{\prime \prime}}\right\| \mid>\xi_{*}^{r} r_{n-3}\left(\xi_{*}\right)} \sup _{\vec{\xi} \in \mathcal{S}_{n}^{\prime}}\left|B_{\mathbf{m}^{\prime \prime}}(\vec{\xi})\right|<\frac{1}{2} \xi_{*}^{-r_{n-3}\left(\xi_{*}\right)}
$$




$$
\sum_{0<\left\|\left|\vec{p}_{\mathbf{m}^{\prime \prime}} \|\right| \leq \xi_{*}^{r_{n-3}\left(\xi_{*}\right)}\right.} \sup _{\vec{\xi} \in \mathcal{G}_{n}^{\prime}}\left|B_{\mathbf{m}^{\prime \prime}}(\vec{\xi})\right|<\frac{1}{2} \xi_{*}^{-r_{n-3}\left(\xi_{*}\right)} .
$$

To prove (463) we first check that

$$
\sup _{\vec{\xi} \in \mathcal{G}_{n}^{\prime}}\left|B_{\mathbf{m}^{\prime \prime}}(\vec{\xi})\right|<\left\|\left|\vec{p}_{\mathbf{m}^{\prime \prime}} \|\right|^{-8} \quad \text { when } \quad\right\|\left|\vec{p}_{\mathbf{m}^{\prime \prime}} \|\right|>\xi_{*}^{r_{n-3}\left(\xi_{*}\right)}
$$

Indeed, for every $\mathbf{m}^{\prime \prime}$ we break $\mathcal{G}_{n}^{\prime}$ into several parts, partition being dependent on $\mathbf{m}^{\prime \prime}$ : $\mathcal{G}_{n}^{\prime}=\cup_{s, s^{\prime}=0}^{n} \mathcal{G}_{s s^{\prime}}$,

$$
\begin{aligned}
& \mathcal{G}_{s s^{\prime}}= \\
& \left\{\vec{\xi} \in \mathcal{G}_{n}^{\prime}:|\vec{\xi}|^{r_{s-1}(|\vec{\xi}|)} \leq \frac{1}{2}\left\|\left|\vec{p}_{\mathbf{m}^{\prime \prime}} \|\right|<\gamma_{s}|\vec{\xi}|^{r_{s}(|\vec{\xi}|)}\right\} \cap\right. \\
& \left\{\vec{\xi} \in \mathcal{G}_{n}^{\prime}:\left|\vec{\xi}+\vec{p}_{\mathbf{m}^{\prime \prime}}\right|^{r_{s^{\prime}-1}\left(\left|\vec{\xi}+\vec{p}_{\mathbf{m}^{\prime \prime}}\right|\right)} \leq \frac{1}{2} \|\left|\vec{p}_{\mathbf{m}^{\prime \prime}}\right|||<\gamma_{s^{\prime}}\left|\vec{\xi}+\vec{p}_{\mathbf{m}^{\prime \prime}}\right|^{r_{s^{\prime}}}\left(\left|\vec{\xi}+\vec{p}_{\mathbf{m}^{\prime \prime}}\right|\right)\right\},
\end{aligned}
$$

where $r_{-1}:=0, r_{0}:=\delta, \gamma_{s}=1$ when $s<n, \gamma_{n}=\infty$. To prove (465), it is enough to show

$$
\sup _{\vec{\xi} \in \mathcal{G}_{s s^{\prime}}}\left|B_{\mathbf{m}^{\prime \prime}}(\vec{\xi})\right|<\left\|\left|\vec{p}_{\mathbf{m}^{\prime \prime}} \|\right|^{-8}\right.
$$

for all $s, s^{\prime}$. Assume $s, s^{\prime}=n$. It follows from (466) that for any $\mathbf{m} \in \mathbb{Z}^{4}$ either $v_{\mathbf{m}}^{(n)}(\vec{\xi})$ or $v_{\mathbf{m}+\mathbf{m}^{\prime \prime}}^{(n)}\left(\vec{\xi}+\vec{p}_{\mathbf{m}^{\prime \prime}}\right)$ is zero. Hence, $\left\langle\mathbf{v}^{(n)}(-\vec{\xi}), \mathbf{v}_{*}^{(n)}\left(-\vec{\xi}-\vec{p}_{\mathbf{m}^{\prime \prime}}\right)\right\rangle=0$, i.e., $B_{\mathbf{m}^{\prime \prime}}(\vec{\xi})=0$. Next, let $0<s<n, s^{\prime}=n$. By (419),

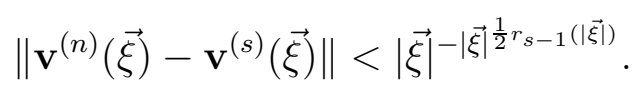

It follows from the definition of $\mathcal{G}_{s n}$ that $\left\langle\mathbf{v}^{(s)}(\vec{\xi}), \mathbf{v}_{*}^{(n)}\left(\vec{\xi}+\vec{p}_{\mathbf{m}^{\prime \prime}}\right)\right\rangle=0$. Therefore,

$$
\left|B_{\mathbf{m}^{\prime \prime}}(\vec{\xi})\right| \leq\left\|\mathbf{v}^{(n)}(\vec{\xi})-\mathbf{v}^{(s)}(\vec{\xi})\right\|<|\vec{\xi}|^{-|\vec{\xi}|^{\frac{1}{2} r_{s-1}(\mid \vec{\xi})}} \quad \text { when } \vec{\xi} \in \mathcal{G}_{s n}
$$

Using (165), (277) and (384), we obtain $\left|B_{\mathbf{m}^{\prime \prime}}(\vec{\xi})\right| \leq|\vec{\xi}|^{-10 r_{s}(|\vec{\xi}|)}$. Considering again the definition of $\mathcal{G}_{s n}$, we get (467). Next, we consider $\mathcal{G}_{0 n}$. By (49), $\mathbf{v}^{(1)}=\mathbf{v}^{(0)}+O\left(|\vec{\xi}|^{-1+44 \mu \delta}\right)$, where $v_{\mathbf{m}}^{(0)}=\delta_{\mathbf{m}, \mathbf{0}}$. By (466), $\left\langle\mathbf{v}^{(0)}(\vec{\xi}), \mathbf{v}^{(n)}\left(\vec{\xi}+\vec{p}_{\mathbf{m}^{\prime \prime}}\right)\right\rangle=0$. Hence, $\left|B_{\mathbf{m}^{\prime \prime}}(\vec{\xi})\right| \leq C|\vec{\xi}|^{-1+44 \mu \delta}$. Using again the definition of $\mathcal{G}_{0 n}$ and the inequality $1-44 \mu \delta>8 \delta$, we obtain (467). The case $s^{\prime}<n$ is considered in the analogous way. Thus, (467) is proved. Summarizing (467) over $\mathbf{m}^{\prime \prime}$, we obtain (463).

Suppose $0<\left\|\left|\vec{p}_{\mathbf{m}^{\prime \prime}} \|\right| \leq \xi_{*}^{r_{n-3}\left(\xi_{*}\right)}\right.$. Let us estimate $B_{\mathbf{m}^{\prime \prime}}(\vec{\xi})$. Assume for definiteness that $\left|\vec{\xi}+\vec{p}_{\mathbf{m}^{\prime \prime}}\right| \leq|\vec{\xi}|$. The case of the opposite inequality is analogous up to the change of the notation $\vec{\xi} \rightarrow \vec{\xi}+\vec{p}_{-\mathbf{m}^{\prime \prime}}$, since $B_{\mathbf{m}^{\prime \prime}}(\vec{\xi})=B_{-\mathbf{m}^{\prime \prime}}\left(\vec{\xi}+\vec{p}_{\mathbf{m}^{\prime \prime}}\right)$. By (460),

$$
H^{(n)}(-\vec{\xi}) \mathbf{v}^{(n)}(-\vec{\xi})=\lambda^{(n)}(-\vec{\xi}) \mathbf{v}^{(n)}(-\vec{\xi})
$$


The analogous relation holds for $\mathbf{v}^{(n)}\left(-\vec{\xi}-\vec{p}_{\mathbf{m}^{\prime \prime}}\right)$ up to the replacement of $H^{(n)}(-\vec{\xi})$ by $H^{(n)}\left(-\vec{\xi}-\vec{p}_{\mathbf{m}^{\prime \prime}}\right)$ and $\lambda^{(n)}(-\vec{\xi})$ by $\lambda^{(n)}\left(-\vec{\xi}-\vec{p}_{\mathbf{m}^{\prime \prime}}\right)$ :

$$
H^{(n)}\left(-\vec{\xi}-\vec{p}_{\mathbf{m}^{\prime \prime}}\right) \mathbf{v}^{(n)}\left(-\vec{\xi}-\vec{p}_{\mathbf{m}^{\prime \prime}}\right)=\lambda^{(n)}\left(-\vec{\xi}-\vec{p}_{\mathbf{m}^{\prime \prime}}\right) \mathbf{v}^{(n)}\left(-\vec{\xi}-\vec{p}_{\mathbf{m}^{\prime \prime}}\right) .
$$

Note that $H^{(n)}\left(-\vec{\xi}-\vec{p}_{\mathbf{m}^{\prime \prime}}\right)$ up to the shift of indices by $-\mathbf{m}^{\prime \prime}$ is equivalent to the operator $P_{\mathbf{m}^{\prime \prime}} H(-\vec{\xi}) P_{\mathbf{m}^{\prime \prime}}$, where $P_{\mathbf{m}^{\prime \prime}}$ is the projection onto the box of the size $\left|\vec{\xi}+\vec{p}_{\mathbf{m}^{\prime \prime}}\right|^{r_{n-1}\left(\left|\vec{\xi}+\vec{p}_{\mathbf{m}^{\prime \prime}}\right|\right)}$ around $-\mathbf{m}^{\prime \prime}$. Using the shifted eigenvector $\mathbf{v}_{*}^{(n)}\left(-\vec{\xi}-\vec{p}_{\mathbf{m}^{\prime \prime}}\right)$, we can rewrite (471) in the form:

$$
P_{\mathbf{m}^{\prime \prime}} H(-\vec{\xi}) P_{\mathbf{m}^{\prime \prime}} \mathbf{V}_{*}^{(n)}\left(-\vec{\xi}-\vec{p}_{\mathbf{m}^{\prime \prime}}\right)=\lambda^{(n)}\left(-\vec{\xi}-\vec{p}_{\mathbf{m}^{\prime \prime}}\right) \mathbf{v}_{*}^{(n)}\left(-\vec{\xi}-\vec{p}_{\mathbf{m}^{\prime \prime}}\right)
$$

where $P_{\mathbf{m}^{\prime \prime}} \mathbf{V}_{*}^{(n)}=\mathbf{v}_{*}^{(n)}$. By (438),

$$
H(-\vec{\xi}) \mathbf{v}^{(n)}(-\vec{\xi})=\lambda^{(n)}(-\vec{\xi}) \mathbf{v}^{(n)}(-\vec{\xi})+O\left(|\xi|^{-|\xi|^{\frac{1}{2} r_{n-1}(|\vec{\xi}|)}}\right)
$$

Similarly,

$$
\begin{aligned}
& H(-\vec{\xi}) \mathbf{v}_{*}^{(n)}\left(-\vec{\xi}-\vec{p}_{\mathbf{m}^{\prime \prime}}\right)= \\
& \lambda^{(n)}\left(-\vec{\xi}-\vec{p}_{\mathbf{m}^{\prime \prime}}\right) \mathbf{v}_{*}^{(n)}\left(-\vec{\xi}-\vec{p}_{\mathbf{m}^{\prime \prime}}\right)+O\left(\left|\xi+\vec{p}_{\mathbf{m}^{\prime \prime}}\right|^{\left.-\left|\xi+\vec{p}_{\mathbf{m}^{\prime \prime}}\right|^{\frac{1}{2} r_{n-1}\left(\left|\vec{\xi}+\vec{p}_{\mathbf{m}^{\prime \prime}}\right|\right)}\right) .}\right.
\end{aligned}
$$

Assume first $\left|\vec{\xi}+\vec{p}_{\mathbf{m}^{\prime \prime}}\right| \leq \frac{1}{2}|\vec{\xi}|$. Then $\left|\lambda^{(n)}(-\vec{\xi})-\lambda^{(n)}\left(-\vec{\xi}-\vec{p}_{\mathbf{m}^{\prime \prime}}\right)\right|>\frac{1}{2}|\vec{\xi}|^{2}$. Using (462), (473) and (474), we obtain:

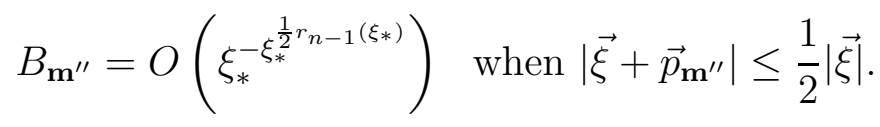

Similar, but somewhat more subtle considerations are required when $\left|\vec{\xi}+\vec{p}_{\mathbf{m}^{\prime \prime}}\right|>\frac{1}{2}|\vec{\xi}|$. We start with introducing a parameter $s$. We will use it to cut $\Omega\left(r_{n-1},|\vec{\xi}|\right)$ to approximately the same size as $\Omega\left(r_{n-1},\left|\vec{\xi}+\vec{p}_{\mathbf{m}^{\prime \prime}}\right|\right)$. If the boxes are of approximately the same size, then $s=n-1$. Indeed, for each $\vec{\xi}$ one of the following relations holds:

$$
|\vec{\xi}|^{r_{s-1}(|\vec{\xi}|)} \leq\left|\vec{\xi}+\vec{p}_{\mathbf{m}^{\prime \prime}}\right|^{r_{n-1}\left(\left|\vec{\xi}+\vec{p}_{\mathbf{m}^{\prime \prime}}\right|\right)}<|\vec{\xi}|^{r_{s}(|\vec{\xi}|)}
$$

where $1 \leq s \leq n-1$ and $s$ is defined by $\mathbf{m}^{\prime \prime}$ and $\vec{\xi}$. Note that $s<n-1$ when $\Omega\left(r_{n-1},|\vec{\xi}|\right)$ is essentially bigger than $\Omega\left(r_{n-1},\left|\vec{\xi}+\vec{p}_{\mathbf{m}^{\prime \prime}}\right|\right)$. Using the second inequality in (476) and (384), we get

$$
\left|\vec{\xi}+\vec{p}_{\mathbf{m}^{\prime \prime}}\right|^{r_{n-3}\left(\left|\vec{\xi}+\vec{p}_{\mathbf{m}^{\prime \prime}}\right|\right)}<\frac{1}{8}|\vec{\xi}|^{r_{s-1}(|\vec{\xi}|)}
$$

Let $P_{\mathbf{0}}$ be the projecting corresponding to $\Omega\left(r_{s},|\vec{\xi}|\right)$. By (419) with $s$ instead of $n$,

$$
\left(I-P_{\mathbf{0} s}\right) \mathbf{v}^{(n)}(-\vec{\xi})=O\left(|\vec{\xi}|^{-|\vec{\xi}|^{\frac{1}{2} r_{s-1}(|\vec{\xi}|)}}\right)
$$


Let us prove the analogous estimate for $\mathbf{v}_{*}^{(n)}$ :

$$
\left(I-P_{\mathbf{0}_{s}}\right) \mathbf{v}_{*}^{(n)}\left(-\vec{\xi}-\vec{p}_{\mathbf{m}^{\prime \prime}}\right)=O\left(|\vec{\xi}|^{-|\vec{\xi}|^{\frac{1}{2} r_{s-1}(|\vec{\xi}|)}}\right) .
$$

Indeed, if $\left(P_{\mathbf{0} s}\right)_{\mathbf{m m}}=0$, then $\left\|\left|\vec{p}_{\mathbf{m}} \|\right|>|\vec{\xi}|^{r_{s-1}(\mid \vec{\xi})}\right.$. Using (477) and the bound on $\|\left|\vec{p}_{\mathbf{m}^{\prime \prime}} \|\right|$, we obtain $\|\left|\vec{p}_{\mathbf{m}+\mathbf{m}^{\prime \prime}}\right|||>\frac{1}{2}|\vec{\xi}|^{r_{s-1}(|\vec{\xi}|)}$. Using (419), (420), we obtain (479). From (473), (474), considering that $\left\|P_{0 s} H\right\|=O\left(|\xi|^{\left.2 r_{s-1}(\mid \vec{\xi})\right)}\right)$ and using (4478), (4479), we get:

$$
\begin{aligned}
& H^{(s)}(-\vec{\xi}) P_{0 s} \mathbf{v}^{(n)}(-\vec{\xi})=\lambda^{(n)}(-\vec{\xi}) P_{0 s} \mathbf{v}^{(n)}(-\vec{\xi})+O\left(|\xi|^{-|\xi|^{\frac{1}{4} r_{s-1}}}\right),
\end{aligned}
$$

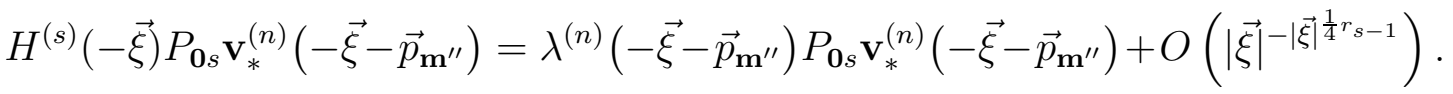

Next, by Theorem 7.17 for step $s,\left|\lambda^{(n)}(-\vec{\xi})-\lambda^{(n)}\left(-\vec{\xi}-\vec{p}_{\mathbf{m}^{\prime \prime}}\right)\right|>\varepsilon_{0}^{(s)} / 2$, where $\varepsilon_{0}^{(s)}=$

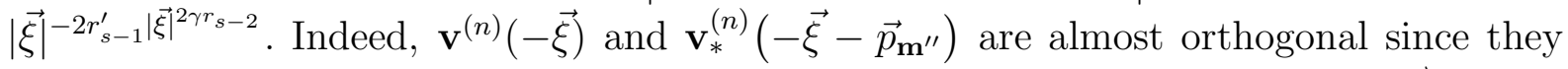
are concentrated around $\mathbf{m}=\mathbf{0}$ and $\mathbf{m}=\mathbf{m}^{\prime \prime} \neq \mathbf{0}$ respectively; thus $\lambda^{(n)}\left(-\vec{\xi}-\vec{p}_{\mathbf{m}^{\prime \prime}}\right)$ must be outside of the interval described in Theorem [7.17, while $\lambda^{(n)}(-\vec{\xi})$ is inside twice shorter interval. Now, using (480) and (481), we obtain:

$$
\left\langle P_{\mathbf{0 s}} \mathbf{v}^{(n)}(-\vec{\xi}), P_{\mathbf{0} s} \mathbf{v}_{*}^{(n)}\left(-\vec{\xi}-\vec{p}_{\mathbf{m}^{\prime \prime}}\right)\right\rangle=O\left(|\vec{\xi}|^{-|\vec{\xi}|^{\frac{1}{4} r_{s-1}}}\right)|\vec{\xi}|^{2 r_{s-1}^{\prime}|\vec{\xi}|^{2 \gamma r_{s-2}}}=O\left(|\vec{\xi}|^{-|\vec{\xi}|^{\frac{1}{8} r_{s-1}}}\right),
$$

see (384). Using one more time (478), (479), and considering (477), we obtain $B_{\mathbf{m}^{\prime \prime}}=$ $O\left(\xi_{*}^{-\xi_{*}^{\frac{1}{8} r_{n-3}\left(\xi_{*}\right)}}\right)$ for the case $\left|\vec{\xi}+\vec{p}_{\mathbf{m}^{\prime \prime}}\right|>\frac{1}{2}|\vec{\xi}|$. Using this estimate together with (475) and considering that the number of $\mathbf{m}^{\prime \prime}$ satisfying $0<\left\|\left|\vec{p}_{\mathbf{m}^{\prime \prime}} \|\right| \leq \xi_{*}^{r_{n-3}\left(\xi_{*}\right)}\right.$ does not exceed $16 \xi_{*}^{4 r_{n-3}\left(\xi_{*}\right)}$, we obtain (464). Substituting the estimates for $B_{\mathbf{m}^{\prime \prime}}$ into (461), we obtain (458).

It is easy to see that $T_{n} \subset S_{n}^{*}$. Therefore, $\left\|T_{n}\right\| \leq 1+o(1)$ and can be extended to the whole space $L_{2}\left(\mathcal{G}_{n}\right)$. We still denote the extended operator by $T_{n}, T_{n}=S_{n}^{*}$. Therefore, $E_{n}$ is a self-adjoint operator.

Lemma 9.4. Let $\mathcal{G}_{n}^{\prime} \subset \mathcal{G}_{n}^{\prime \prime} \subset \mathcal{G}_{n}$. The following relation holds as $n \rightarrow \infty$ :

$$
E_{n}\left(\mathcal{G}_{n}^{\prime}\right) E_{n}\left(\mathcal{G}_{n}^{\prime \prime}\right)=E_{n}\left(\mathcal{G}_{n}^{\prime}\right)+o(1),
$$

where o(1) is taken in the space of bounded operators and uniform in $\mathcal{G}_{n}^{\prime}, \mathcal{G}_{n}^{\prime \prime}$.

Corollary 9.5. $E_{n}\left(\mathcal{G}_{n}^{\prime \prime}\right) E_{n}\left(\mathcal{G}_{n}^{\prime}\right)=E_{n}\left(\mathcal{G}_{n}^{\prime}\right)+o(1)$.

This corollary is valid, since $E_{n}$ is selfajoint. 
Corollary 9.6. $E_{n}^{2}\left(\mathcal{G}_{n}^{\prime}\right)=E_{n}\left(\mathcal{G}_{n}^{\prime}\right)+o(1)$ for any $\mathcal{G}_{n}^{\prime} \subset \mathcal{G}_{n}$.

Proof. Let $I_{n}\left(\mathcal{G}_{n}^{\prime}\right)$ be the projection from $L_{2}\left(\mathcal{G}_{n}^{\prime \prime}\right)$ to $L_{2}\left(\mathcal{G}_{n}^{\prime}\right)$. It is easy to see that $T_{n}\left(\mathcal{G}_{n}^{\prime}\right)=I_{n}\left(\mathcal{G}_{n}^{\prime}\right) T_{n}\left(\mathcal{G}_{n}^{\prime \prime}\right)$. Hence, $T_{n}\left(\mathcal{G}_{n}^{\prime}\right) S_{n}\left(\mathcal{G}_{n}^{\prime \prime}\right)=I_{n}\left(\mathcal{G}_{n}^{\prime}\right) T_{n}\left(\mathcal{G}_{n}^{\prime \prime}\right) S_{n}\left(\mathcal{G}_{n}^{\prime \prime}\right)$. By (458) for set $\mathcal{G}_{n}^{\prime \prime}, T_{n}\left(\mathcal{G}_{n}^{\prime \prime}\right) S_{n}\left(\mathcal{G}_{n}^{\prime \prime}\right)=i d\left(\mathcal{G}_{n}^{\prime \prime}\right)+o(1)$, where $i d\left(\mathcal{G}_{n}^{\prime \prime}\right)$ is the identity in $L_{2}\left(\mathcal{G}_{n}^{\prime \prime}\right)$. It immediately follows $T_{n}\left(\mathcal{G}_{n}^{\prime}\right) S_{n}\left(\mathcal{G}_{n}^{\prime \prime}\right)=I_{n}\left(\mathcal{G}_{n}^{\prime}\right)+o(1)$. Substituting the last relation into the formula $E_{n}\left(\mathcal{G}_{n}^{\prime}\right) E_{n}\left(\mathcal{G}_{n}^{\prime \prime}\right)=S_{n}\left(\mathcal{G}_{n}^{\prime}\right) T_{n}\left(\mathcal{G}_{n}^{\prime}\right) S_{n}\left(\mathcal{G}_{n}^{\prime \prime}\right) T_{n}\left(\mathcal{G}_{n}^{\prime \prime}\right)$, we obtain (482).

Let

$$
\mathcal{G}_{n, \lambda}=\left\{\vec{\varkappa} \in \mathcal{G}_{n}: \lambda^{(n)}(\vec{\varkappa})<\lambda\right\} .
$$

This set is Lebesgue measurable, since $\mathcal{G}_{n}$ is open and $\lambda^{(n)}(\vec{\varkappa})$ is continuous on $\mathcal{G}_{n}$.

Lemma 9.7. $\left|\mathcal{G}_{n, \lambda+\varepsilon} \backslash \mathcal{G}_{n, \lambda}\right| \leq 2 \pi \varepsilon$ when $0 \leq \varepsilon \leq 1$.

The proof is based on Lemma 7.21 and completely analogous to that of Lemma 2 in [31].

By (454), $E_{n}\left(\mathcal{G}_{n, \lambda+\varepsilon}\right)-E_{n}\left(\mathcal{G}_{n, \lambda}\right)=E_{n}\left(\mathcal{G}_{n, \lambda+\varepsilon} \backslash \mathcal{G}_{n, \lambda}\right)$. Let us obtain an estimate for this projection.

Lemma 9.8. For any $F \in C_{0}^{\infty}\left(\mathbb{R}^{2}\right)$ and $0 \leq \varepsilon \leq 1$,

$$
\left\|\left(E_{n}\left(\mathcal{G}_{n, \lambda+\varepsilon}\right)-E_{n}\left(\mathcal{G}_{n, \lambda}\right)\right) F\right\|_{L_{2}\left(\mathbb{R}^{2}\right)}^{2} \leq C(F) \epsilon
$$

where $C(F)$ is uniform with respect to $n$ and $\lambda$.

Proof. Let $\mathcal{G}_{n}^{\prime}=\mathcal{G}_{n, \lambda+\varepsilon} \backslash \mathcal{G}_{n, \lambda}$. Using the definition (455) of $E_{n}$ and formula (459) with $f=g=T_{n} F$, we obtain

$$
\left\|E_{n}\left(\mathcal{G}_{n}^{\prime}\right) F\right\|_{L_{2}\left(\mathbb{R}^{2}\right)}^{2}<(1+o(1))\left\|T_{n} F\right\|_{L_{\infty}\left(\mathcal{G}_{n}^{\prime}\right)}^{2}\left|\mathcal{G}_{n}^{\prime}\right| .
$$

Using (456) and Corollary 8.8 we easily get $\left\|T_{n} F\right\|_{L_{\infty}\left(\mathcal{G}_{n}^{\prime}\right)}<2\|F\|_{L_{1}\left(\mathbb{R}^{2}\right)}$. Substituting this estimate into (485) and using Lemma 9.7, we obtain (484).

\subsection{Sets $\mathcal{G}_{\infty}$ and $\mathcal{G}_{\infty, \lambda}$}

By construction, $\mathcal{G}_{n+1} \subset \mathcal{G}_{n}, \mathcal{G}_{\infty}=\bigcap_{n=1}^{\infty} \mathcal{G}_{n}$. Therefore, the perturbation formulas for $\lambda^{(n)}(\vec{\varkappa})$ and $\Psi_{n}(\vec{\varkappa})$ hold in $\mathcal{G}_{\infty}$ for all $n$. Let

$$
\mathcal{G}_{\infty, \lambda}=\left\{\vec{\varkappa} \in \mathcal{G}_{\infty}: \lambda_{\infty}(\vec{\varkappa})<\lambda\right\} .
$$

The function $\lambda_{\infty}(\vec{\varkappa})$ is a Lebesgue measurable function, since it is a limit of the sequence of measurable functions. Hence, the set $\mathcal{G}_{\infty, \lambda}$ is measurable.

Lemma 9.9. The measure of the symmetric difference of two sets $\mathcal{G}_{\infty, \lambda}$ and $\mathcal{G}_{n, \lambda}$ converges to zero as $n \rightarrow \infty$ uniformly in $\lambda$ in every bounded interval:

$$
\lim _{n \rightarrow \infty}\left|\mathcal{G}_{\infty, \lambda} \Delta \mathcal{G}_{n, \lambda}\right|=0 .
$$

The proof is completely analogous to the proof of Lemma 4 in [31]. 


\subsection{Projections $E\left(\mathcal{G}_{\infty, \lambda}\right)$}

In this section, we show that the operators $E_{n}\left(\mathcal{G}_{\infty, \lambda}\right)$ have a strong limit $E_{\infty}\left(\mathcal{G}_{\infty, \lambda}\right)$ in $L_{2}\left(\mathbb{R}^{2}\right)$ as $n$ tends to infinity. The operator $E_{\infty}\left(\mathcal{G}_{\infty, \lambda}\right)$ is a spectral projection of $H$. It can be represented in the form $E_{\infty}\left(\mathcal{G}_{\infty, \lambda}\right)=S_{\infty} T_{\infty}$, where $S_{\infty}$ and $T_{\infty}$ are strong limits of $S_{n}\left(\mathcal{G}_{\infty, \lambda}\right)$ and $T_{n}\left(\mathcal{G}_{\infty, \lambda}\right)$, respectively. For any $F \in C_{0}^{\infty}\left(\mathbb{R}^{2}\right)$, we show:

$$
\begin{gathered}
E_{\infty}\left(\mathcal{G}_{\infty, \lambda}\right) F=\frac{1}{4 \pi^{2}} \int_{\mathcal{G}_{\infty, \lambda}}\left(F, \Psi_{\infty}(\vec{\varkappa})\right) \Psi_{\infty}(\vec{\varkappa}, \vec{x}) d \vec{\varkappa}, \\
H E_{\infty}\left(\mathcal{G}_{\infty, \lambda}\right) F=\frac{1}{4 \pi^{2}} \int_{\mathcal{G}_{\infty, \lambda}} \lambda_{\infty}(\vec{\varkappa})\left(F, \Psi_{\infty}(\vec{\varkappa})\right) \Psi_{\infty}(\vec{\varkappa}, \vec{x}) d \vec{\varkappa} .
\end{gathered}
$$

Using properties of $E_{\infty}\left(\mathcal{G}_{\infty, \lambda}\right)$, we prove absolute continuity of the branch of the spectrum corresponding to functions $\Psi_{\infty}(\vec{\varkappa})$.

We consider the sequence of operators $S_{n}\left(\mathcal{G}_{\infty, \lambda}\right)$ which are given by (457) with $\mathcal{G}_{n}^{\prime}=$ $\mathcal{S}_{\infty, \lambda}$.

Lemma 9.10. We have

$$
\left\|\left(S_{n}\left(\mathcal{G}_{\infty, \lambda}\right)-S_{n-1}\left(\mathcal{G}_{\infty, \lambda}\right)\right) f\right\|_{L_{2}\left(\mathbb{R}^{2}\right)}<C\|f\|_{L_{2}\left(\mathcal{G}_{\infty, \lambda}\right)} \xi_{*}^{-\frac{1}{4} \xi_{*}^{r_{n-2}\left(\xi_{*}\right)}}, \quad n \geq 3
$$

Proof. Considering as in the proof of Lemma 9.1, we obtain

$$
\begin{gathered}
\left\|\left(S_{n}-S_{n-1}\right) f\right\|_{L_{2}\left(\mathbb{R}^{2}\right)}^{2}=\left\|\widehat{S_{n} f}-\widehat{S_{n-1} f}\right\|_{L_{2}\left(\mathbb{R}^{2}\right)}^{2}= \\
\int_{\mathbb{R}^{2}} \sum_{\mathbf{m}^{\prime \prime}} \tilde{B}_{\mathbf{m}^{\prime \prime}}(\vec{\xi}) f(-\vec{\xi}) \bar{f}\left(-\vec{\xi}-\vec{p}_{\mathbf{m}^{\prime \prime}}\right) \chi\left(\mathcal{G}_{\infty, \lambda},-\vec{\xi}\right) \chi\left(\mathcal{G}_{\infty, \lambda},-\vec{\xi}-\vec{p}_{\mathbf{m}^{\prime \prime}}\right) d \vec{\xi} \\
\tilde{B}_{\mathbf{m}^{\prime \prime}}(\vec{\xi})=\sum_{\mathbf{m} \in \Omega\left(r_{n-1}, \mid \vec{\xi}\right): \mathbf{m}+\mathbf{m}^{\prime \prime} \in \Omega\left(r_{n-1},\left|\vec{\xi}+\vec{p}_{\mathbf{m}^{\prime \prime}}\right|\right)}\left(v_{\mathbf{m}}^{(n)}-v_{\mathbf{m}}^{(n-1)}\right)(-\vec{\xi}) \overline{\left(v_{\mathbf{m}+\mathbf{m}^{\prime \prime}}^{(n)}-v_{\mathbf{m}+\mathbf{m}^{\prime \prime}}^{(n-1)}\right)}(-\vec{\xi}
\end{gathered}
$$

Assume for definiteness that $\left|\vec{\xi}+\vec{p}_{\mathbf{m}^{\prime \prime}}\right| \leq|\vec{\xi}|$. If $\left\|\left|\vec{p}_{\mathbf{m}^{\prime \prime}} \|\right|>2|\vec{\xi}|^{r_{n-1}(|\vec{\xi}|)}\right.$, then $\tilde{B}_{\mathbf{m}^{\prime \prime}}(\vec{\xi})=0$. Let $\vec{\xi}:\left\|\left|\vec{p}_{\mathbf{m}^{\prime \prime}} \|\right| \leq 2|\vec{\xi}|^{r_{n-1}(|\vec{\xi}|)}\right.$. Using (419) with $n$ instead of $n+1$, we easily obtain:

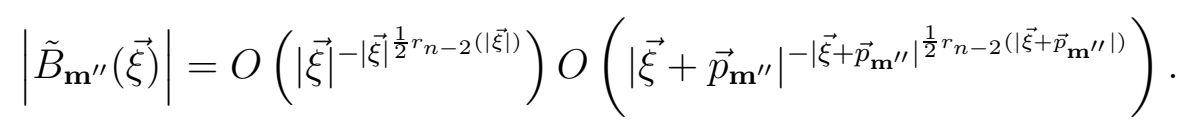

Considering (384) with $n-1$ instead of and $n$ and taking into account that $\left\|\left|\vec{p}_{\mathbf{m}^{\prime \prime}} \|\right| \leq\right.$ $2|\vec{\xi}|^{r_{n-1}(|\vec{\xi}|)}$, we easily get:

$$
\left|\tilde{B}_{\mathbf{m}^{\prime \prime}}(\vec{\xi})\right|=\left\|\left|\vec{p}_{\mathbf{m}^{\prime \prime}}\right|\right\|^{-8} O\left(\xi_{*}^{-\xi_{*}^{\frac{1}{2} r_{n-2}\left(\xi_{*}\right)}}\right) .
$$


Summarizing the last estimate for $\mathbf{m}^{\prime \prime} \neq \mathbf{0}$ and using (491) for $\mathbf{m}^{\prime \prime}=\mathbf{0}$, we arrive at (489).

By (489), the sequence of operators $S_{n}\left(\mathcal{G}_{\infty, \lambda}\right)$ is a Cauchy sequence in the space of bounded operators. We denote its limit by $S_{\infty}\left(\mathcal{G}_{\infty, \lambda}\right)$. Note that the convergence of $S_{n}\left(\mathcal{G}_{\infty, \lambda}\right)$ to $S_{\infty}\left(\mathcal{G}_{\infty, \lambda}\right)$ is uniform in $\lambda$ when $\lambda>\lambda_{*}$.

Lemma 9.11. The operator $S_{\infty}\left(\mathcal{G}_{\infty, \lambda}\right)$ can be described by the formula

$$
\left(S_{\infty} f\right)(\vec{x})=\frac{1}{2 \pi} \int_{\mathcal{G}_{\infty, \lambda}} f(\vec{\varkappa}) \Psi_{\infty}(\vec{\varkappa}, \vec{x}) d \vec{\varkappa}
$$

for any $f \in L_{\infty}\left(\mathcal{G}_{\infty, \lambda}\right)$.

Proof. From Theorem 8.9 it follows that for every $f \in L_{\infty}\left(\mathcal{G}_{\infty, \lambda}\right)$

$$
\lim _{n \rightarrow \infty} \int_{\mathcal{S}_{\infty, \lambda}} f(\vec{\varkappa}) \Psi_{n}(\vec{\varkappa}, \vec{x}) d \vec{\varkappa}=\int_{\mathcal{G}_{\infty, \lambda}} f(\vec{\varkappa}) \Psi_{\infty}(\vec{\varkappa}, \vec{x}) d \vec{\varkappa}
$$

for all $\vec{x}$. Hence, (492) holds.

Now we consider the sequence of operators $T_{n}\left(\mathcal{G}_{\infty, \lambda}\right)$ which are given by (456) and act from $L_{2}\left(\mathbb{R}^{2}\right)$ to $L_{2}\left(\mathcal{G}_{\infty, \lambda}\right)$. Since, $T_{n}=S_{n}^{*}$, the sequence has a limit $T_{\infty}$ in the class of bounded operators, $T_{\infty}=S_{\infty}^{*}$. Note that the convergence of $T_{n}\left(\mathcal{G}_{\infty, \lambda}\right)$ to $T_{\infty}\left(\mathcal{G}_{\infty, \lambda}\right)$ is uniform in $\lambda$ when $\lambda>\lambda_{*}$.

Lemma 9.12. The operator $T_{\infty}\left(\mathcal{G}_{\infty, \lambda}\right)$ can be described by the formula $T_{\infty}\left(\mathcal{G}_{\infty, \lambda}\right) F=$ $\frac{1}{2 \pi}\left(F, \Psi_{\infty}(\vec{\varkappa})\right)$ for any $F \in C_{0}^{\infty}\left(\mathbb{R}^{2}\right)$.

Proof. The lemma easily follows from Theorem 8.9 and formula (456).

Lemma 9.13. Operators $E_{n}\left(\mathcal{G}_{\infty, \lambda}\right)$ have a limit $E_{\infty}\left(\mathcal{G}_{\infty, \lambda}\right)$ in the class of bounded operators in $L_{2}\left(\mathbb{R}^{2}\right)$, the convergence being uniform for $\lambda>\lambda_{*}$. The operator $E_{\infty}\left(\mathcal{G}_{\infty, \lambda}\right)$ is a projection. For any $F \in C_{0}^{\infty}\left(\mathbb{R}^{2}\right)$ it is given by (487).

Proof. The lemma immediately follows from convergence of sequences $S_{n}, T_{n}$ and Lemmas 9.4, 9.11, 9.12.

Lemma 9.14. There is a strong limit $E_{\infty}\left(\mathcal{G}_{\infty}\right)$ of the projections $E_{\infty}\left(\mathcal{G}_{\infty, \lambda}\right)$ as $\lambda$ goes to infinity.

Corollary 9.15. The operator $E_{\infty}\left(\mathcal{G}_{\infty}\right)$ is a projection.

Proof. It can be easily seen from (487) that the sequence of $E_{\infty}\left(\mathcal{G}_{\infty, \lambda}\right)$ is monotonuos in $\lambda$. It is well known that a monotone sequence of projectors has a limit.

The proofs of the next two lemmas are completely analogous to the proofs of Lemmas 10,11 in 31 . 
Lemma 9.16. Projections $E_{\infty}\left(\mathcal{G}_{\infty, \lambda}\right), \lambda \in \mathbb{R}$, and $E_{\infty}\left(\mathcal{G}_{\infty}\right)$ reduce the operator $H$.

Lemma 9.17. The family of projections $E_{\infty}\left(\mathcal{G}_{\infty, \lambda}\right)$ is the resolution of the identity of the operator $H E_{\infty}\left(\mathcal{G}_{\infty}\right)$ acting in $E_{\infty}\left(\mathcal{G}_{\infty}\right) L_{2}\left(\mathbb{R}^{2}\right)$.

Lemma 9.18. Formula (488) holds, when $F \in C_{0}^{\infty}\left(\mathbb{R}^{2}\right)$.

Proof. By the previous lemma, $E_{\infty}\left(\mathcal{G}_{\infty, \lambda}\right) F \in D(H)$. It is easy to see that the r.h.s. of (487) can be differentiated with respect to $\vec{x}$ under the integral sign. Now, considering (446), we get (488).

\subsection{Proof of Absolute Continuity}

Now we show that the branch of spectrum (semi-axis) corresponding to $\mathcal{G}_{\infty}$ is absolutely continuous.

Theorem 9.19. For any $F \in C_{0}^{\infty}\left(\mathbb{R}^{2}\right)$ and $0 \leq \varepsilon \leq 1$,

$$
\left|\left(E_{\infty}\left(\mathcal{G}_{\infty, \lambda+\varepsilon}\right) F, F\right)-\left(E_{\infty}\left(\mathcal{G}_{\infty, \lambda}\right) F, F\right)\right| \leq C_{F} \varepsilon .
$$

Corollary 9.20. The spectrum of the operator $H E_{\infty}\left(\mathcal{G}_{\infty}\right)$ is absolutely continuous.

Proof. By formula (487),

$$
\left|\left(E_{\infty}\left(\mathcal{G}_{\infty, \lambda+\varepsilon}\right) F, F\right)-\left(E\left(\mathcal{G}_{\infty, \lambda}\right) F, F\right)\right| \leq C_{F}\left|\mathcal{G}_{\infty, \lambda+\varepsilon} \backslash \mathcal{G}_{\infty, \lambda}\right| .
$$

Applying Lemmas 9.7 and 9.9, we immediately get (494).

\section{Appendices}

\subsection{Appendix 1. Proof of Lemma 3.12}

Proof.

1. The case $p_{\mathbf{m}}>4 k$. From (73) it immediately follows that $\left|\Im \varphi_{\mathbf{m}}^{ \pm}\right|>(\cosh )^{-1} 2>1$. Hence, $\mathcal{W}_{0} \cap \mathcal{O}_{\mathbf{m}}(k, \tau)=\emptyset$.

Further we use the Taylor series for $\left|\vec{k}(\varphi)+\vec{p}_{\mathbf{m}}\right|_{\mathbb{R}}^{2}-k^{2}$ near its zeros: Noting that

$$
\left|\vec{k}(\varphi)+\vec{p}_{\mathbf{m}}\right|_{\mathbb{R}}^{2}-k^{2}=2 k p_{\mathbf{m}} \cos \left(\varphi-\varphi_{\mathbf{m}}\right)+p_{\mathbf{m}}^{2}
$$

and recalling that $\varphi_{\mathbf{m}}^{ \pm}$are the solutions of $\left|\vec{k}(\varphi)+\vec{p}_{\mathbf{m}}\right|_{\mathbb{R}}^{2}=k^{2}$, we see:

$$
\cos \left(\varphi_{\mathbf{m}}^{ \pm}-\varphi_{\mathbf{m}}\right)=-\frac{p_{\mathbf{m}}}{2 k}, \quad\left|\sin \left(\varphi_{\mathbf{m}}^{ \pm}-\varphi_{\mathbf{m}}\right)\right|=\sqrt{\left|1-\frac{p_{\mathbf{m}}^{2}}{4 k^{2}}\right|} .
$$


Expanding (495) around $\varphi_{\mathbf{m}}^{ \pm}$, we get:

$$
\begin{aligned}
& \left|\vec{k}(\varphi)+\vec{p}_{\mathbf{m}}\right|_{\mathbb{R}}^{2}-k^{2}= \\
& -2 k p_{\mathbf{m}} \sin \left(\varphi_{\mathbf{m}}^{ \pm}-\varphi_{\mathbf{m}}\right) r_{\mathbf{m}}\left(1+O\left(r_{\mathbf{m}}^{2}\right)\right)+k p_{\mathbf{m}} \cos \left(\varphi_{\mathbf{m}}^{ \pm}-\varphi_{\mathbf{m}}\right) r_{\mathbf{m}}^{2}\left(1+O\left(r_{\mathbf{m}}^{2}\right)\right)
\end{aligned}
$$

where $r_{\mathbf{m}}=\left|\varphi-\varphi_{\mathbf{m}}^{ \pm}\right|$.

2. In the second case we put $r_{\mathbf{m}}=\frac{\tau k^{-1+\delta_{*}}}{p_{\mathbf{m}} \sqrt{\left|1-\frac{p_{\mathbf{m}}^{2}}{4 k^{2}}\right|}}(1+o(1))$ when $k^{-1+2 \delta_{*}}<p_{\mathbf{m}}<$ $4 k$ and $\left|1-\frac{p_{\mathbf{m}}^{2}}{4 k^{2}}\right|>\tau k^{-2+\delta_{*}}$. Substituting $r_{\mathbf{m}}$ into (497), we get that the modulus of the first term is $2 \tau k^{\delta_{*}}(1+o(1))$ and that of the second term is $\frac{\tau^{2} k^{-2+2 \delta_{*}}}{2\left|1-\frac{p_{m}^{2}}{4 k^{2}}\right|}(1+o(1))$. Using the condition $\left|1-\frac{p_{\mathrm{m}}^{2}}{4 k^{2}}\right|>\tau k^{-2+\delta_{*}}$, one can easily see that the former is at least twice greater than the latter. Thus, we get

$$
|| \vec{k}(\varphi)+\left.\vec{p}_{\mathbf{m}}\right|_{\mathbb{R}} ^{2}-k^{2} \mid>\tau k^{\delta_{*}} \text { when }\left|\varphi-\varphi_{\mathbf{m}}^{ \pm}\right|=r_{\mathbf{m}}
$$

Now, the minimum principle yield that this inequality holds everywhere outside the $\operatorname{discs} \cup_{ \pm, j \in \mathbb{Z}}\left(\Phi_{\mathbf{m}}^{ \pm}+2 \pi j\right)$. Hence, $\mathcal{O}_{\mathbf{m}} \subset \cup_{ \pm, j \in \mathbb{Z}}\left(\Phi_{\mathbf{m}}^{ \pm}+2 \pi j\right)$.

3. In the third case we put $r_{\mathbf{m}}=32 \tau k^{-1+\delta_{*} / 2}(1+o(1))$ and $\left|1-\frac{p_{\mathbf{m}}^{2}}{4 k^{2}}\right|<\tau k^{-2+\delta_{*}}$. This time the modulus of the second term in (497) is $64 \cdot 32 \tau^{2} k^{\delta_{*}}(1+o(1))$ and that of the first is smaller than $128 \tau^{3 / 2} k^{\delta_{*}}(1+o(1))$. Therefore we again have (498) and $\mathcal{O}_{\mathbf{m}} \subset \cup_{ \pm, j \in \mathbb{Z}}\left(\Phi_{\mathbf{m}}^{ \pm}+2 \pi j\right)$.

\subsection{Appendix 2. Proof of Lemma 3.13}

Proof. By definition of $\mathcal{M}^{\prime}\left(\varphi_{0}\right), \varphi_{0} \in \cap_{j=0}^{2} \mathcal{O}_{\mathbf{m}_{j}}(k, 1)$. By (72),

$$
|| \vec{k}\left(\varphi_{0}\right)+\left.\vec{p}_{\mathbf{m}_{j}}\right|^{2}-k^{2} \mid \leq k^{\delta_{*}}, j=0,1,2
$$

It follows:

$$
\left|2\left(\vec{k}\left(\varphi_{0}\right)+\vec{p}_{\mathbf{m}_{0}}, \vec{p}_{\mathbf{q}_{j}}\right)+p_{\mathbf{q}_{j}}^{2}\right|<2 k^{\delta_{*}}, \mathbf{q}_{j}=\mathbf{m}_{j}-\mathbf{m}_{0}, \quad j=1,2,
$$

where $\left\|\left|\vec{p}_{\mathbf{q}_{j}} \|\right| \leq 3 j k^{\delta}\right.$. We will complete the proof by the way of contradiction. Assume that $\vec{p}_{\mathbf{q}_{1}}$ and $\vec{p}_{\mathbf{q}_{2}}$ are not colinear. We represent every $\vec{p}_{\mathbf{q}_{j}}$ in the form: $\vec{p}_{\mathbf{q}_{j}}=2 \pi\left(\mathbf{s}_{j}+\alpha \mathbf{s}_{j}^{\prime}\right)$, 
$\mathbf{s}_{j}, \mathbf{s}_{j}^{\prime} \in \mathbb{Z}^{2},\left|\mathbf{s}_{j}\right|,\left|\mathbf{s}_{j}^{\prime}\right|<8 k^{\delta}$ and denote $[\mathbf{a}, \mathbf{b}]=a_{1} b_{2}-a_{2} b_{1}$. It follows from (499) that the angle (modulo $\pi$ ) between $\vec{p}_{\mathbf{q}_{1}}, \vec{p}_{\mathbf{q}_{2}}$ is less than $k^{-1+\delta_{*}+2 \delta \mu}$. Hence,

$$
\left[\vec{p}_{\mathbf{q}_{1}}, \vec{p}_{\mathbf{q}_{2}}\right]=O\left(k^{-1+\delta_{*}+2(1+\mu) \delta}\right) .
$$

Substituting $\vec{p}_{\mathbf{q}_{j}}=2 \pi\left(\mathbf{s}_{j}+\alpha \mathbf{s}_{j}^{\prime}\right), j=1,2$, we obtain:

$$
n_{1}+\alpha p_{1}+\alpha^{2} m_{1}=O\left(k^{-1+\delta_{*}+2(1+\mu) \delta}\right)
$$

where $n_{1}, p_{1}, m_{1}$ are integers, $n_{1}=\left[\mathbf{s}_{1}, \mathbf{s}_{2}\right], p_{1}=\left[\mathbf{s}_{1}, \mathbf{s}_{2}^{\prime}\right]+\left[\mathbf{s}_{1}^{\prime}, \mathbf{s}_{2}\right], m_{1}=\left[\mathbf{s}_{1}^{\prime}, \mathbf{s}_{2}^{\prime}\right]$. Obviously, $n_{1}, p_{1}, m_{1}=O\left(k^{2 \delta}\right)$. Next, we use the condition (25) on $\alpha$ at the beginning of the paper. Assume first, $0 \leq\left|n_{1}\right|+\left|p_{1}\right|+\left|m_{1}\right| \leq N_{1}$. If $k$ is sufficiently large, $k>k_{0}\left(N_{0}, N_{1}, \delta, \delta_{*} ; \alpha\right)$, the inequality (501) yields $n_{1}+\alpha p_{1}+\alpha^{2} m_{1}=0$. This means vectors $\vec{p}_{\mathbf{q}_{1}}, \vec{p}_{\mathbf{q}_{2}}$ are colinear and the lemma is proved. Let $\left|n_{1}\right|+\left|p_{1}\right|+\left|m_{1}\right|>N_{1}$. Then either, again, $n_{1}+\alpha p_{1}+\alpha^{2} m_{1}=$ 0 and we are done, or (501) contradicts the initial condition (25) on $\alpha$ when $2 \delta N_{0} \leq 1 / 2$ and $k$ is sufficiently large: $k>k_{0}\left(N_{0}, N_{1}, \delta, \delta_{*} ; \alpha\right)$.

\subsection{Appendix 3}

Lemma 10.1. If the $\|\mid \cdot\|$-distance between $\mathcal{M}_{2}^{j, s}$ and $\mathcal{M}_{2}^{j, s^{\prime}}$ is less than $L(\geq 1)$, then the $\|\mid \cdot\|$-distance between central points of $\mathcal{M}_{2}^{j, s}$ and $\mathcal{M}_{2}^{j, s^{\prime}}$ is less than $C(Q) L$. Moreover, $\mathcal{M}_{2}^{j, s}$ belongs to $\left(C(Q) L+\left\|\left|\vec{p}_{\mathbf{q}} \|\right|\right)\right.$-neighborhood of $\mathcal{M}_{2}^{j, s^{\prime}}$ and vise versa.

Proof. Let $\mathbf{m}+\hat{n} \mathbf{q} \in \mathcal{M}_{2}^{j, s}, \mathbf{m}^{\prime}+\hat{n}^{\prime} \mathbf{q} \in \mathcal{M}_{2}^{j, s^{\prime}}$ and $\left\|\left|\vec{p}_{\mathbf{m}+\hat{n} \mathbf{q}-\mathbf{m}^{\prime}-\hat{n}^{\prime} \mathbf{q}} \|\right| \leq L\right.$ (in particular, $\left.p_{\mathbf{m}+\hat{n} \mathbf{q}-\mathbf{m}^{\prime}-\hat{n}^{\prime} \mathbf{q}} \leq 2 \pi L\right)$. Note that $\vec{p}_{\mathbf{m}-\mathbf{m}^{\prime}}$ is colinear with $\vec{p}_{\mathbf{q}}$, since $\mathbf{m}, \mathbf{m}^{\prime} \in \mathcal{M}_{2}^{j}$, and $p_{\mathbf{m}-\mathbf{m}^{\prime}}<p_{\mathbf{q}}$, since $\mathbf{m}, \mathbf{m}^{\prime}$ are central points, see (79). Considering the last inequality, we obtain, $\left|\hat{n}-\hat{n}^{\prime}\right| \leq C_{0}(Q) L$ and, thus, $\left\|\left|\vec{p}_{\mathbf{m}-\mathbf{m}^{\prime}} \|\right| \leq C(Q) L\right.$.

Let $\mathbf{m}+n \mathbf{q} \in \mathcal{M}_{2}^{j, s}$. We prove that there is a point of $\mathcal{M}_{2}^{j, s^{\prime}}$ in the $\left(C(Q) L+\left\|\left|\vec{p}_{\mathbf{q}} \|\right|\right)\right.$ neighborhood of $\mathbf{m}+n \mathbf{q}$. Using (80), it is easy to see that $\mathbf{m}^{\prime}+n \mathbf{q}$ is in the $\left\|\left|\vec{p}_{\mathbf{q}} \|\right|-\right.$ neighborhood of $\mathcal{M}_{2}^{j, s^{\prime}}$. Considering that $\left\|\left|\vec{p}_{\mathbf{m}-\mathbf{m}^{\prime}} \|\right| \leq C(Q) L\right.$, we finish the proof of the lemma.

\subsection{Appendix 4}

Lemma 10.2. The equation

$$
\lambda^{(1)}\left(\vec{\varkappa}^{(1)}(\varphi)+\vec{p}_{\mathbf{m}}\right)=k^{2}+\varepsilon_{0}, \quad 0<p_{\mathbf{m}} \leq 4 k^{\delta},\left|\varepsilon_{0}\right| \leq p_{\mathbf{m}} k^{\delta},
$$

has no more than two solutions $\varphi^{ \pm}\left(\varepsilon_{0}\right)$ in $\tilde{\mathcal{W}}^{(1)}\left(k, \frac{1}{8}\right) \cap \mathcal{O}_{\mathbf{m}}\left(k, \frac{1}{2}\right)$. They satisfy the estimates:

$$
\left|\varphi^{ \pm}\left(\varepsilon_{0}\right)-\varphi_{\mathbf{m}}^{ \pm}\right|<k^{-1+2 \delta}
$$


Proof. Let $\varphi \in \tilde{\mathcal{W}}^{(1)}\left(k, \frac{1}{4}\right) \cap \mathcal{O}_{\mathbf{m}}\left(k, \frac{1}{2}\right)$. The equation (502) is equivalent to

$$
\lambda^{(1)}(\vec{y}(\varphi))=\lambda^{(1)}\left(\vec{y}(\varphi)-\vec{p}_{\mathbf{m}}\right)+\varepsilon_{0}, \quad \vec{y}(\varphi)=\vec{\varkappa}^{(1)}(\varphi)+\vec{p}_{\mathbf{m}} .
$$

We use perturbation formula (42):

$$
|\vec{y}(\varphi)|_{\mathbb{R}}^{2}+f_{1}(\vec{y}(\varphi))=\left|\vec{y}(\varphi)-\vec{p}_{\mathbf{m}}\right|_{\mathbb{R}}^{2}+f_{1}\left(\vec{y}(\varphi)-\vec{p}_{\mathbf{m}}\right)+\varepsilon_{0},
$$

where $f_{1}$ is the series in the right-hand side of (42). This equation can be rewritten as

$$
\left(2\left(\vec{\varkappa}^{(1)}(\varphi), \vec{p}_{\mathbf{m}}\right)_{\mathbb{R}}+p_{\mathbf{m}}^{2}\right)+f_{1}(\vec{y}(\varphi))-f_{1}\left(\vec{y}(\varphi)-\vec{p}_{\mathbf{m}}\right)=\varepsilon_{0} .
$$

Using the notation $\vec{p}_{\mathbf{m}}=p_{\mathbf{m}}\left(\cos \varphi_{\mathbf{m}}, \sin \varphi_{\mathbf{m}}\right)$, dividing both sides of the equation (504) by $2 p_{\mathbf{m}} k$, and considering that $\vec{y}(\varphi)=\vec{\varkappa}^{(1)}(\varphi)+\vec{p}_{\mathbf{m}}=\left(k+h^{(1)}\right) \vec{\nu}+\vec{p}_{\mathbf{m}}$, we obtain:

$$
\cos \left(\varphi-\varphi_{\mathbf{m}}\right)+\frac{p_{\mathbf{m}}}{2 k}-\varepsilon_{0} g_{1}(\varphi)+g_{2}(\varphi)+g_{3}(\varphi)=0
$$

where $g_{1}(\varphi)=\left(2 p_{\mathbf{m}} k\right)^{-1}$ and

$$
g_{2}(\varphi)=\frac{\left(\vec{h}^{(1)}(\varphi), \vec{p}_{\mathbf{m}}\right)}{p_{\mathbf{m}} k}, \quad g_{3}(\varphi)=\left(f_{1}(\vec{y}(\varphi))-f_{1}\left(\vec{y}(\varphi)-\vec{p}_{\mathbf{m}}\right)\right) g_{1}(\varphi), \quad \vec{h}^{(1)}(\varphi)=h^{(1)}(\varphi) \vec{\nu}
$$

Using Lemma 3.11 and considering that $0<p_{\mathbf{m}} \leq 4 k^{\delta}$, we easily obtain:

$$
\left|g_{2}(\varphi)\right|=\left|\frac{\left(\vec{h}^{(1)}(\varphi), \vec{p}_{\mathbf{m}}\right)}{p_{\mathbf{m}} k}\right| \leq \frac{2 h^{(1)}}{k}=O\left(k^{-4+(80 \mu+6) \delta}\right) .
$$

Let us show $g_{3}(\varphi)=O\left(k^{-1+\delta}\right)$. If $p_{\mathbf{m}} \geq k^{-2+\delta(80 \mu+6)}$, then the estimate easily follows from (48) and the estimate for $g_{1}$. Let $p_{\mathbf{m}}<k^{-2+\delta(80 \mu+6)}$. It can be easily shown that the series $f_{1}(\vec{y}), \nabla f_{1}(\vec{y})$ converge for all $\vec{y}:\left|\vec{y}-\vec{\varkappa}^{(1)}(\varphi)\right|_{\mathbb{C}^{2}}=O\left(k^{-\delta(40 \mu+1)}\right)$ or $\left|\vec{y}+\vec{p}_{\mathbf{m}}-\vec{\varkappa}^{(1)}(\varphi)\right|_{\mathbb{C}^{2}}=$ $O\left(k^{-\delta(40 \mu+1)}\right)$ (see Lemma 3.5), the series being holomorphic with respect to $y_{1}, y_{2}$ in these neighborhoods. Using (48) (cf. Lemma 3.5), we get $\nabla f_{1}(\vec{y})=O\left(k^{-2+\delta(120 \mu+7)}\right)$. Hence,

$$
\left|f_{1}(\vec{y}(\varphi))-f_{1}\left(\vec{y}(\varphi)-\vec{p}_{\mathbf{m}}\right)\right| \leq \sup \left|\nabla f_{1}\right| p_{\mathbf{m}}=o\left(p_{\mathbf{m}}\right),
$$

and therefore, $g_{3}(\varphi)=O\left(k^{-1+\delta}\right)$. Since $\left|\varepsilon_{0}\right|<p_{\mathbf{m}} k^{\delta}$, we obtain $\varepsilon_{0} g_{1}(\varphi)=O\left(k^{-1+\delta}\right)$. Thus,

$$
g_{2}(\varphi)+g_{3}(\varphi)-\varepsilon_{0} g_{1}(\varphi)=O\left(k^{-1+\delta}\right) \text { when } \varphi \in \tilde{\mathcal{W}}^{(1)}\left(k, \frac{1}{4}\right) \cap \mathcal{O}_{\mathbf{m}}\left(k, \frac{1}{2}\right) .
$$

By definition $\varphi_{\mathbf{m}}^{ \pm}$satisfy the equation $\cos \left(\varphi-\varphi_{\mathbf{m}}\right)+\frac{p_{\mathbf{m}}}{2 k}=0$.

Suppose both $\varphi_{\mathbf{m}}^{ \pm}$are in $\tilde{\mathcal{W}}^{(1)}\left(k, \frac{3}{16}\right)$. We draw two circles $C_{ \pm}$centered at $\varphi_{\mathbf{m}}^{ \pm}$with the radius $k^{-1+2 \delta}$. They are both inside $\tilde{\mathcal{W}}^{(1)}\left(k, \frac{1}{4}\right) \cap \mathcal{O}_{\mathbf{m}}\left(k, \frac{1}{2}\right)$, the perturbation series 
converging and the estimate (506) holds. For any $\varphi$ on $C_{ \pm},\left|\varphi-\varphi_{\mathbf{m}}^{ \pm}\right|=k^{-1+2 \delta}$ and, therefore, $\left|\cos \left(\varphi-\varphi_{\mathbf{m}}\right)+\frac{p_{\mathbf{m}}}{2 k}\right|>\frac{1}{2} k^{-1+2 \delta}>\left|g_{2}(\varphi)+g_{3}(\varphi)-\varepsilon_{0} g_{1}(\varphi)\right|$ for any $\varphi \in C_{ \pm}$. By Rouché's Theorem, there is only one solution of the equation (505) inside each $C_{ \pm}$. Obviously, (505) does not have solutions in $\tilde{\mathcal{W}}^{(1)}\left(k, \frac{1}{4}\right) \cap \mathcal{O}_{\mathbf{m}}\left(k, \frac{1}{2}\right)$ outside $C_{ \pm}$.

If both $\varphi_{\mathbf{m}}^{ \pm}$are not in $\tilde{\mathcal{W}}^{(1)}\left(k, \frac{3}{16}\right)$, then their distance to $\tilde{\mathcal{W}}^{(1)}\left(k, \frac{1}{8}\right)$ is at least $\frac{1}{16} k^{-\delta(40 \mu+1)}$, hence $\left|\cos \left(\varphi-\varphi_{\mathbf{m}}\right)+\frac{p_{\mathbf{m}}}{2 k}\right|>\frac{1}{4} k^{-1+2 \delta}$ in $\tilde{\mathcal{W}}^{(1)}\left(k, \frac{1}{8}\right)$. Therefore, equation (505) has no solution in $\tilde{\mathcal{W}}^{(1)}\left(k, \frac{1}{8}\right) \cap \mathcal{O}_{\mathbf{m}}\left(k, \frac{1}{2}\right)$. The case, when only one $\varphi_{\mathbf{m}}^{ \pm}$is not in $\tilde{\mathcal{W}}^{(1)}\left(k, \frac{3}{16}\right)$ is the obvious combination of the two previous situations. Thus, there are at most two solutions in $\tilde{\mathcal{W}}^{(1)}\left(k, \frac{1}{8}\right) \cap \mathcal{O}_{\mathbf{m}}\left(k, \frac{1}{2}\right)$ and $\left|\varphi^{ \pm}\left(\varepsilon_{0}\right)-\varphi_{\mathbf{m}}^{ \pm}\right|<k^{-1+2 \delta}$.

Lemma 10.3. For any $\varphi \in \tilde{\mathcal{W}}^{(1)}\left(k, \frac{1}{4}\right) \cap \mathcal{O}_{\mathbf{m}}(k, 1)$ satisfying the estimate $\left|\varphi-\varphi_{\mathbf{m}}^{ \pm}\right|<k^{-\delta}$,

$$
\frac{\partial}{\partial \varphi} \lambda^{(1)}\left(\vec{\varkappa}^{(1)}(\varphi)+\vec{p}_{\mathbf{m}}\right)= \pm 2 p_{\mathbf{m}} k(1+o(1))
$$

Proof. First, assume $\varphi$ is real. Let $\vec{y}(\varphi)=\vec{\varkappa}^{(1)}(\varphi)+\vec{p}_{\mathbf{m}}$. Using the perturbation formula (42) and Lemma 3.11, we obtain:

$$
\begin{gathered}
\frac{\partial}{\partial \varphi} \lambda^{(1)}(\vec{y}(\varphi))=\frac{\partial}{\partial \varphi}\left[\lambda^{(1)}(\vec{y}(\varphi))-k^{2 l}\right]=\frac{\partial}{\partial \varphi}\left[\lambda^{(1)}(\vec{y}(\varphi))-\lambda^{(1)}\left(\vec{y}(\varphi)-\vec{p}_{\mathbf{m}}\right)\right]= \\
\left(\nabla_{\vec{y}} \lambda^{(1)}(\vec{y}(\varphi))-\nabla_{\vec{y}} \lambda^{(1)}\left(\vec{y}(\varphi)-\vec{p}_{\mathbf{m}}\right), \frac{\partial}{\partial \varphi} \vec{y}(\varphi)\right)_{\mathbb{R}}= \\
\left(\nabla|\vec{y}(\varphi)|_{\mathbb{R}}^{2}-\nabla\left|\vec{y}(\varphi)-\vec{p}_{\mathbf{m}}\right|_{\mathbb{R}}^{2},\left(k+h^{(1)}\right) \vec{t}+\left(h^{(1)}\right)^{\prime} \vec{\nu}\right)_{\mathbb{R}}+ \\
\left(\nabla f_{1}(\vec{y}(\varphi))-\nabla f_{1}\left(\vec{y}(\varphi)-\vec{p}_{\mathbf{m}}\right),\left(k+h^{(1)}\right) \vec{t}+\left(h^{(1)}\right)^{\prime} \vec{\nu}\right)_{\mathbb{R}},
\end{gathered}
$$

where $\vec{\nu}=(\cos \varphi, \sin \varphi)$ and $\vec{t}=\vec{\nu}^{\prime}=(-\sin \varphi, \cos \varphi), f_{1}$ is the series in the right-hand side of (42). Note that

$$
\nabla|\vec{y}(\varphi)|_{\mathbb{R}}^{2}-\nabla\left|\vec{y}(\varphi)-\vec{p}_{\mathbf{m}}\right|_{\mathbb{R}}^{2}=2 \vec{p}_{\mathbf{m}}
$$

Substituting (509) into (508), we get $\frac{\partial}{\partial \varphi} \lambda^{(1)}(\vec{y}(\varphi))=T_{1}+T_{2}$,

$$
\begin{aligned}
T_{1} & =2\left(\vec{p}_{\mathbf{m}},\left(k+h^{(1)}\right) \vec{t}+\left(h^{(1)}\right)^{\prime} \vec{\nu}\right)_{\mathbb{R}}, \\
T_{2} & =\left(\nabla f_{1}(\vec{y}(\varphi))-\nabla f_{1}\left(\vec{y}(\varphi)-\vec{p}_{\mathbf{m}}\right),\left(k+h^{(1)}\right) \vec{t}+\left(h^{(1)}\right)^{\prime} \vec{\nu}\right)_{\mathbb{R}} .
\end{aligned}
$$

We see that $\varphi$ is close to $\varphi_{\mathbf{m}} \pm \pi / 2$, since $\left|\varphi-\varphi_{\mathbf{m}}^{ \pm}\right|<k^{-\delta}$ by the hypothesis of the lemma and

$$
\varphi_{\mathbf{m}}^{ \pm}=\varphi_{\mathbf{m}} \pm \pi / 2+O\left(k^{-1+\delta}\right) \text { when } p_{\mathbf{m}}<4 k^{\delta} .
$$

Now we readily obtain: $\left(\vec{p}_{\mathbf{m}}, \vec{\nu}\right)_{\mathbb{R}}=o\left(p_{\mathbf{m}}\right),\left(\vec{p}_{\mathbf{m}}, \vec{t}\right)_{\mathbb{R}}= \pm p_{\mathbf{m}}(1+o(1))$. Using also estimates (68), (69) for $h^{(1)}$, we get $T_{1}= \pm 2 p_{\mathbf{m}} k(1+o(1))$. Let us estimate $T_{2}$. As above, 
the series $f_{1}(\vec{y}), \nabla f_{1}(\vec{y}), D^{2} f_{1}(\vec{y})$ converge for all $\vec{y}:\left|\vec{y}-\vec{\varkappa}^{(1)}(\varphi)\right|_{\mathbb{C}^{2}}=O\left(k^{-\delta(40 \mu+1)}\right)$ or $\left|\vec{y}+\vec{p}_{\mathbf{m}}-\vec{\varkappa}^{(1)}(\varphi)\right|_{\mathbb{C}^{2}}=O\left(k^{-\delta(40 \mu+1)}\right)$ the series being holomorphic with respect to $y_{1}, y_{2}$ in these neighborhoods, and we have $\nabla f_{1}(\vec{y})=O\left(k^{-2+\delta(120 \mu+7)}\right), D^{2} f_{1}(\vec{y})=O\left(k^{-2+\delta(160 \mu+8)}\right)$. Let $p_{\mathbf{m}} \geq k^{-2+\delta(120 \mu+8)}$. Then, using the estimate for $\nabla f_{1}(\vec{y})$, we easily obtain $T_{2}=$ $O\left(k^{-1+\delta(120 \mu+7)}\right)=o\left(k p_{\mathbf{m}}\right)$. Let $p_{\mathbf{m}}<k^{-2+\delta(120 \mu+8)}$. Then, using the estimate for the second derivative in the direction of $\vec{p}_{\mathbf{m}}$, we get $\left|\nabla f_{1}(\vec{y}(\varphi))-\nabla f_{1}\left(\vec{y}(\varphi)-\vec{p}_{\mathbf{m}}\right)\right|=$ $O\left(p_{\mathbf{m}} k^{-2+(160 \mu+8) \delta}\right)$. Therefore, $T_{2}=O\left(p_{\mathbf{m}} k^{-1+(160 \mu+8) \delta}\right)$. Thus, $T_{2}=o\left(k p_{\mathbf{m}}\right)$ for all $\vec{p}_{\mathbf{m}}$. Adding the estimates for $T_{1}, T_{2}$, we get (507).

Since all formulas can be analytically extended to the area of non-real $\varphi$, the estimates being preserved, (507) holds for any $\varphi \in \tilde{\mathcal{W}}^{(1)}\left(k, \frac{1}{4}\right) \cap \mathcal{O}_{\mathbf{m}}(k, 1)$.

Lemma 10.4. Let $\tilde{\mathcal{O}}_{\mathbf{m}, \varepsilon}^{ \pm}$be the open discs of the radius $\varepsilon$ centered at $\varphi^{ \pm}(0)$ defined in Lemma 10.2. For any $\varphi \in \tilde{\mathcal{W}}^{(1)}\left(k, \frac{1}{8}\right) \cap \mathcal{O}_{\mathbf{m}}\left(k, \frac{1}{2}\right), \varphi \notin \tilde{\mathcal{O}}_{\mathbf{m}, \varepsilon}^{ \pm}$, and $0 \leq \varepsilon<k^{-1+\delta}$,

$$
\left|\lambda^{(1)}(\vec{y}(\varphi))-k^{2}\right| \geq k p_{\mathbf{m}} \varepsilon \text {. }
$$

Proof. Suppose (511) does not hold for some $\varphi \in \tilde{\mathcal{W}}^{(1)}\left(k, \frac{1}{8}\right) \cap \mathcal{O}_{\mathbf{m}}\left(k, \frac{1}{2}\right), \varphi \notin \tilde{\mathcal{O}}_{\mathbf{m}, \varepsilon}^{ \pm}$. This means that $\varphi$ satisfies equation (502) with some $\varepsilon_{0}$ : $\left|\varepsilon_{0}\right|<k p_{\mathbf{m}} \varepsilon\left(<p_{\mathbf{m}} k^{\delta}\right)$. By Lemma 10.2, $\varphi$ could be either $\varphi^{+}\left(\varepsilon_{0}\right)$ or $\varphi^{-}\left(\varepsilon_{0}\right)$. Without loss of generality, assume $\varphi=\varphi^{+}\left(\varepsilon_{0}\right)$. By Lemma 10.2, $\left|\varphi^{+}\left(\varepsilon_{0}\right)-\varphi_{\mathbf{m}}^{ \pm}\right|<k^{-1+2 \delta}$ for $\varphi_{\mathbf{m}}^{+}$or $\varphi_{\mathbf{m}}^{-}$. By the same lemma and (510), there is a single $\varphi^{+}(0)$ in the $2 k^{-1+2 \delta}$-neighborhood of $\varphi^{+}\left(\varepsilon_{0}\right)$. Obviously, the $2 k^{-1+2 \delta_{-}}$ neighborhood of $\varphi^{+}\left(\varepsilon_{0}\right)$ satisfies conditions of Lemma 10.3. Considering (507), we obtain $\left|\varphi^{+}\left(\varepsilon_{0}\right)-\varphi^{+}(0)\right|<\varepsilon$, i.e., $\varphi \in \tilde{\mathcal{O}}_{\mathbf{m}, \varepsilon}$. This contradicts the hypothesis of the lemma.

Lemma 10.5. If $0<p_{\mathbf{m}} \leq 4 k^{\delta}$ and $\varphi \in \tilde{\mathcal{W}}^{(1)}\left(k, \frac{1}{8}\right) \cap \mathcal{O}_{\mathbf{m}}\left(k, \frac{1}{2}\right)$, then

$$
\left\|\left(\lambda^{(1)}(\vec{y}(\varphi))-k^{2}\right)\left(P_{\mathbf{m}}\left(H\left(\vec{\varkappa}^{(1)}(\varphi)\right)-k^{2}\right) P_{\mathbf{m}}\right)^{-1}\right\| \leq 8, \quad \vec{y}(\varphi):=\vec{\varkappa}^{(1)}(\varphi)+\vec{p}_{\mathbf{m}} .
$$

Proof. Let $\tilde{C}_{1}$ be the circle in $\mathbb{C}$ of the radius $\frac{1}{4} k^{1-40 \mu \delta}$ centered at $z_{0}=|\vec{y}(\varphi)|_{\mathbb{R}}^{2}$. Using (110) and (111), we easily get:

$$
|| \vec{y}(\varphi)+\left.\vec{p}_{\mathbf{q}}\right|_{\mathbb{R}} ^{2}-z \mid \gtrsim \frac{1}{4} k^{1-40 \mu \delta}, \quad \text { when } \mathbf{q} \in \Omega(\delta), \quad z \in \tilde{C}_{1} .
$$

Therefore,

$$
\begin{gathered}
\left\|\left(P_{\mathbf{m}}\left(H_{0}\left(\vec{\varkappa}^{(1)}(\varphi)\right)-z\right) P_{\mathbf{m}}\right)^{-1}\right\| \lesssim 4 k^{-1+40 \mu \delta}, \\
\left\|\left(P_{\mathbf{m}}\left(H_{0}\left(\vec{\varkappa}^{(1)}(\varphi)\right)-z\right) P_{\mathbf{m}}\right)^{-1}\right\|_{1} \lesssim 4 k^{-1+40 \delta(\mu+1)} .
\end{gathered}
$$

Next, by (106),

$$
\left|\operatorname{det} \frac{P_{\mathbf{m}}\left(H\left(\vec{\varkappa}^{(1)}(\varphi)\right)-z\right) P_{\mathbf{m}}}{P_{\mathbf{m}}\left(H_{0}\left(\vec{\varkappa}^{(1)}(\varphi)\right)-z\right) P_{\mathbf{m}}}-1\right|<2\|V\|\left\|\left(P_{\mathbf{m}}\left(H_{0}\left(\vec{\varkappa}^{(1)}(\varphi)\right)-z\right) P_{\mathbf{m}}\right)^{-1}\right\|_{1}
$$


for every $z$ on the contour $\tilde{C}_{1}$. Using the estimate (514), we obtain that the righthand part of (515) is less than 1. Applying Rouché's Theorem, we conclude that the determinant has the same number of zeros and poles inside $\tilde{C}_{1}$. Considering that the resolvent $\left(P_{\mathbf{m}}\left(H_{0}\left(\vec{\varkappa}^{(1)}(\varphi)\right)-z\right) P_{\mathbf{m}}\right)^{-1}$ has a single pole, $z=|\vec{y}(\varphi)|_{\mathbb{R}}^{2}$, we obtain that $\left(P_{\mathbf{m}}\left(H\left(\vec{\varkappa}^{(1)}(\varphi)\right)-z\right) P_{\mathbf{m}}\right)^{-1}$ has a single pole inside $\tilde{C}_{1}$ too. Obviously, the pole is at the point $z=\lambda^{(1)}(\vec{y}(\varphi))$. Therefore

$$
\left(\lambda^{(1)}(\vec{y}(\varphi))-z\right)\left(P_{\mathbf{m}}\left(H\left(\vec{\varkappa}^{(1)}(\varphi)\right)-z\right) P_{\mathbf{m}}\right)^{-1}
$$

is a holomorphic function of $z$ inside $\tilde{C}_{1}$.

Let $z \in \tilde{C}_{1}$. Using (48), we easily obtain: $\left|\lambda^{(1)}(\vec{y}(\varphi))-z\right| \leq k^{1-40 \mu \delta}$. From (513) and Hilbert identity it follows that

$$
\left\|\left(P_{\mathbf{m}}\left(H\left(\vec{\varkappa}^{(1)}(\varphi)\right)-z\right) P_{\mathbf{m}}\right)^{-1}\right\| \leq 8 k^{-1+40 \mu \delta}, \text { when } z \in \tilde{C}_{1} .
$$

Multiplying the last two estimates, and using maximum principle we get

$$
\left\|\left(\lambda^{(1)}(\vec{y}(\varphi))-z\right)\left(P_{\mathbf{m}}\left(H\left(\vec{\varkappa}^{(1)}(\varphi)\right)-z\right) P_{\mathbf{m}}\right)^{-1}\right\| \leq 8, \quad z \in \overline{\operatorname{Int} \tilde{C}_{1}} .
$$

Note that $z:=k^{2} \in \overline{\operatorname{Int} \tilde{C}_{1}}$. Indeed, by (110), $\left.|| \vec{y}(\varphi)\right|_{\mathbb{R}} ^{2}-k^{2} \mid<\frac{1}{2} k^{\delta_{*}}<\frac{1}{4} k^{1-40 \mu \delta}$. Substituting $z=k^{2}$ in the last estimate, we get (512).

\subsection{Appendix 5}

First we rewrite $D_{i_{0}}\left(\tau_{1}\right)$ in the form: $D_{i_{0}}=\operatorname{det}\left(E(\vec{\varkappa})\left(H(\vec{\varkappa})-k^{2}\right) E(\vec{\varkappa})\right)$, where $\vec{\varkappa}=$ $\left(\tau_{1}, \beta_{1} \tau_{1}+\beta_{2}\right), \tau_{1,0}-\sigma_{\text {large }} / 100<\tau_{1}<\tau_{1,0}+\sigma_{\text {large }} / 100, E(\vec{\varkappa})$ is the spectral projection corresponding to $\lambda_{i_{0}}(\vec{\varkappa})$ and $\lambda_{i_{0}+1}(\vec{\varkappa})$. Thus, $E P=P E=E, P$ being the projector associated with $\mathcal{M}_{*}$ under consideration. By $E_{0}$ we denote the corresponding spectral projection for $V=0$.

Proposition 10.6. The operators $E_{0}(\vec{\varkappa})$, and $H_{0}(\vec{\varkappa})$ can be extended as holomorphic functions of $\tau_{1}$ to $T_{0}$. They have the following properties:

$$
\begin{gathered}
E_{0} V E_{0}=0, \\
\left\|E_{0}\left(H_{0}-k^{2}\right)^{-1}\right\|=O\left(\sigma_{\text {large }}^{-2}\right) \quad \text { on } \partial T_{0}, \\
\left\|\left(P-E_{0}\right)\left(H_{0}-k^{2}\right)^{-1}\right\|=O\left(\Lambda^{-1 / 2} p_{\mathbf{q}}^{-1}\right) \quad \text { in } T_{0},
\end{gathered}
$$

The spectral projection $E(\vec{\varkappa}), \vec{\varkappa}=\left(\tau_{1}, \beta_{1} \tau_{1}+\beta_{2}\right), \tau_{1,0}-\sigma_{\text {large }} / 100<\tau_{1}<\tau_{1,0}+$ $\sigma_{\text {large }} / 100$, obeys the following asymptotics:

$$
E={ }_{\Lambda \rightarrow \infty} E_{0}+O\left(\frac{\|V\|_{\infty}}{\Lambda^{1 / 2} p_{\mathbf{q}}}\right),
$$




$$
\left(E-E_{0}\right)\left(H-k^{2}\right)=_{\Lambda \rightarrow \infty} O\left(\|V\|_{\infty}\right)
$$

Here, the constants in $O(\cdot)$ do not depend on $V, \Lambda$ or $p_{\mathbf{q}}$.

The projection $E(\vec{\varkappa})$ can be extended as a holomorphic function of $\tau_{1}$ to $T_{0}$, the estimates (521), (522) are preserved in $T_{0}$.

Proof. Estimates (519) and (520) easily follow from the definitions of $E_{0}, T_{0}$ and properties of $H_{0}(\vec{\varkappa})$. In particular, (519) follows from the fact that $H_{0}(\vec{\varkappa})$ is a quadratic polynomial with respect to $\tau_{1}$. Next, for $V=0$ the eigenvalues $\tilde{\lambda}_{i_{0}}, \tilde{\lambda}_{i_{0}+1}$ are equal to $\left(\tau_{1}+n_{1} p_{\mathbf{q}}\right)^{2}$ and $\left(\tau_{1}+n_{2} p_{\mathbf{q}}\right)^{2}$, where $n_{1}, n_{2}$ have different signs and $\left|\left(n_{1}-n_{2}\right) p_{\mathbf{q}}\right|>\frac{1}{4} \Lambda^{1 / 2}$. It follows that $V_{n_{1}-n_{2}}=0$, since $V$ is a trigonometric polynomial (here we assume that $\left.\Lambda^{1 / 2}>4 Q p_{\mathbf{q}}\right)$. Considering also that $V_{\mathbf{0}}=0$, we obtain $E_{0} V E_{0}=0$.

Assume now that $\Lambda^{1 / 2}>4\|V\|_{\infty} p_{\mathbf{q}}^{-1}$. Formulas (521), (522) follow from a perturbation expansion for $E$ :

$$
E=E_{0}+\sum_{r=1}^{\infty} \hat{G}^{(r)}, \quad \hat{G}^{(r)}=\frac{(-1)^{r+1}}{2 \pi i} \int_{\hat{C}}\left(H_{0}-z\right)^{-1}\left(V\left(H_{0}-z\right)^{-1}\right)^{r} d z
$$

where $\hat{C}$ is the contour around the pair of eigenvalues of the distance $R=\frac{1}{2} \Lambda^{1 / 2} p_{\mathbf{q}}$ from them. The r.h.s. of (523) can be easily extended as a holomorphic function of $\tau_{1}$ to $T_{0}$. Therefore, the estimates (521), (522) are preserved in $T_{0}$.

Lemma 10.7. The determinant $D_{i_{0}}\left(\tau_{1}\right)$ has the same number $J$ of zeros in $T_{0}$ as $D_{i_{0}, 0}\left(\tau_{1}\right)$, $T_{0}$ being the $\sigma_{\text {large }} / 100$-neighborhood of the zeros of $D_{i_{0}, 0}$, and

$$
\left|D_{i_{0}}\left(\tau_{1}\right)\right|>\left(\sigma_{\text {large }} / 100\right)^{J} / 2 \quad \text { on } \partial T_{0} .
$$

Proof. First, we prove that

$$
\operatorname{det}\left(P\left(H(\vec{\varkappa})-k^{2} I\right) P\right)\left(P\left(H_{0}(\vec{\varkappa})-k^{2} I\right) P\right)^{-1}=1+O\left(\frac{Q\|V\|_{\infty}}{\sigma_{\text {large }} p_{\mathbf{q}}^{1 / 2} \Lambda^{1 / 4}}\right) \text {, when } \tau_{1} \in \partial T_{0} \text {. }
$$

Indeed, this determinant can be rewritten in the form $\operatorname{det}(I+S)$, where

$$
S=\left(P\left(H_{0}(\vec{\varkappa})-k^{2} I\right) P\right)^{-1 / 2} V\left(P\left(H_{0}(\vec{\varkappa})-k^{2} I\right) P\right)^{-1 / 2} .
$$

By (1518), $E_{0} S E_{0}=0$. Using now (1519), (1520), we obtain $\|S\|_{1}=O\left(\frac{Q\|V\|_{\infty}}{\sigma_{\text {large }} p_{\mathbf{q}}^{1 / 2} \Lambda^{1 / 4}}\right)$. The last estimate yields (525). Now, by (107) and Rouché's Theorem, $\operatorname{det}\left(P\left(H(\vec{\varkappa})-k^{2} I\right) P\right)$ and $\operatorname{det}\left(P\left(H_{0}(\vec{\varkappa})-k^{2} I\right) P\right)$ have the same number of zeros in $T_{0}$, when $\Lambda$ is sufficiently large: $\Lambda=\Lambda(V)$. 
Next, note that the determinant of operator $\left(I-E_{0}\right) P\left(H_{0}(\vec{\varkappa})-k^{2} I\right) P$ has no zeros inside $T_{0}$, see (520). Considering (519) $-(522)$, it is not difficult to show that

$$
\frac{\operatorname{det}\left((I-E) P\left(H(\vec{\varkappa})-k^{2} I\right) P\right)}{\operatorname{det}\left(\left(I-E_{0}\right) P\left(H_{0}(\vec{\varkappa})-k^{2} I\right) P\right)}=1+O\left(\frac{\|V\|_{\infty}}{p_{\mathbf{q}} \Lambda^{1 / 2}}\right) \text {, when } \tau_{1} \in \partial T_{0} \text {. }
$$

It follows that $\operatorname{det}\left((I-E) P\left(H(\vec{\varkappa})-k^{2} I\right) P\right)$ has no zeros inside $T_{0}$. Now, we obtain from (525) and (526) that

$$
\frac{D_{i_{0}}}{D_{i_{0}, 0}}=1+O\left(\frac{Q\|V\|_{\infty}}{\sigma_{\text {large }} p_{\mathbf{q}}^{1 / 2} \Lambda^{1 / 4}}\right), \text { when } \tau_{1} \in \partial T_{0},
$$

and, hence (choosing $\Lambda=\Lambda(V)$ to be sufficiently large), $D_{i_{0}, 0}$ and $D_{i_{0}}$ have the same number of zeros inside $T_{0}$ and even twice smaller neighborhood. Since $D_{i_{0}, 0}$ satisfies (238), $D_{i_{0}}$ satisfies the analogous estimate.

\subsection{Appendix 6}

Lemma 10.8. Let $R$ be the smallest positive integer for which (310) holds. We have $R>k^{\left(\frac{\gamma}{2}+2 \delta_{0}\right) r_{1}-\delta_{*}-2 \delta}$.

Proof. Notice that

$$
\mathcal{A}_{R}=\sum_{i_{1}, \ldots, i_{R}=0}^{\hat{I}} \mathcal{A}_{i_{1}, \ldots, i_{R}}
$$

where

$$
\mathcal{A}_{i_{1}, \ldots, i_{R}}:=P^{\partial}\left(\hat{H}_{0}-k^{2}\right)^{-1} W\left[\prod_{r=1}^{R}\left(P_{i_{r}}\left(\hat{H}_{0}-k^{2}\right)^{-1} W\right)\right] P^{(i n t)},
$$

$P_{i}$ being as in (307), $i \geq 0$. Here we used that $R$ is the smallest positive integer for which $\mathcal{A}_{R} \neq 0$.

In principal, everything is defined by the case where all $i_{q}$ are equal to zero. However, to include impurities of non-resonant and white clusters, we need an additional construction. Consider a particular $\mathcal{A}_{i_{1}, \ldots, i_{R}}$. From the sequence $i_{1}, \ldots, i_{R}$ we take a subsequence of all non-zero indices $i_{r_{1}}, \ldots, i_{r_{s}}, 1 \leq r_{1}<\cdots<r_{s} \leq R$ (this sequence can be empty). In this subsequence we construct a subsubsequence $j_{1}, \ldots, j_{p}(p \leq s)$ of non-repeating indices as follows. We choose $j_{1}=i_{r_{1}}$. If $i_{r_{1}}$ is not equal to any other $i_{r_{t}}, t=2, \ldots, s$, then $j_{2}=i_{r_{2}}$. If there is one or more $i_{r_{t}}$ equal to $i_{r_{1}}$ then we denote the segment between the first and the last $i_{r_{1}}$ as $I_{1}$, both ends being included. The next term after $I_{1}$ we choose to be an $j_{2}$ etc. Thus, with a slight abuse of the notation we have:

$$
i_{r_{1}}, \ldots, i_{r_{s}}=I_{1}, I_{2}, \ldots, I_{p}, \quad p \leq s,
$$


where $I_{q}$ may contain just one element. Now, the initial sequence $i_{1}, \ldots, i_{R}$ can be represented as $I_{1}^{0}, \tilde{I}_{1}, I_{2}^{0}, \tilde{I}_{2}, \ldots, \tilde{I}_{p}, I_{p+1}^{0}$, where each $I_{q}^{0}$ is a sequence of only zeros (it can be empty) and $\tilde{I}_{q}$ is $I_{q}$ with possibly some zeros inside. Put

$$
P_{j_{q}} B P_{j_{q}}:=P_{j_{q}}\left(\hat{H}_{0}-k^{2}\right)^{-1} W P_{i^{\prime}}\left(\hat{H}_{0}-k^{2}\right)^{-1} W \ldots\left(\hat{H}_{0}-k^{2}\right)^{-1} W P_{j_{q}} .
$$

Here for all internal projectors $P_{i^{\prime}}$ we have either $i^{\prime} \in I_{q}$ or $i^{\prime}=0$, all indices in $\tilde{I}_{q}$ being included. We notice that $P_{j_{q}} B P_{j_{q}}$ has a block form. Now we can represent $\mathcal{A}_{i_{1}, \ldots, i_{R}}$ as follows:

$$
\begin{aligned}
& \mathcal{A}_{i_{1}, \ldots, i_{R}}=P^{\partial}\left(\hat{H}_{0}-k^{2}\right)^{-1} W\left[\prod_{q=1}^{p}\left(P_{0}\left(\hat{H}_{0}-k^{2}\right)^{-1} W\right)^{s_{q}} P_{j_{q}} B P_{j_{q}}\left(\hat{H}_{0}-k^{2}\right)^{-1} W\right] \\
& \times\left(P_{0}\left(\hat{H}_{0}-k^{2}\right)^{-1} W\right)^{s_{p+1}} P^{(i n t)},
\end{aligned}
$$

where $s_{q}$ is the number of elements in $I_{q}^{0}, s_{q} \geq 0 ; j_{q}$ is a non-zero index corresponding to $I_{q}$. Obviously, $\left(\left(\hat{H}_{0}-k^{2}\right)^{-1} W\right)_{\mathbf{m m}^{\prime}}=0$ if $\|\mid \cdot\|$-distance between the cluster containing $\vec{p}_{\mathbf{m}}$ and the cluster containing $\vec{p}_{\mathbf{m}^{\prime}}$ is greater than $k^{\delta}$ (here, as usual, we consider the points in the range of $P_{0}$ as $1 \times 1$ clusters). Next, if $P_{j_{q}}$ is the projection on a non-resonant cluster, then

$$
\left(P_{j_{q}} B P_{j_{q}}\right)_{\mathbf{m m}^{\prime}}=0, \quad \text { for }\left\|\left|\vec{p}_{\mathbf{m}-\mathbf{m}^{\prime}} \|\right|>k^{\delta_{*}+\delta},\right.
$$

since a non-resonant cluster has the size not greater than $k^{\delta_{*}+\delta}$. Let $p^{\prime}$ be the number of non-resonant projections in the sequence $\left\{P_{j_{q}}\right\}_{q=1}^{p}$. Hence, $p-p^{\prime}$ is the number of white clusters. The operator $\mathcal{A}_{i_{1}, \ldots, i_{R}}$ can be non-zero only if

$$
\frac{D}{2} \leq k^{\delta} \sum_{q=1}^{p+1}\left(s_{q}+1\right)+k^{\delta_{*}+\delta} p^{\prime}+\sum_{m=1}^{p-p^{\prime}} d_{m},
$$

where $\frac{D}{2}$ is the $\left\||\cdot \||\right.$ distance between the supports of $P^{\partial}$ and $P^{(i n t)}$ and $d_{m}$ is the size of a white cluster. Next, we prove that

$$
\sum_{q=1}^{p+1}\left(s_{q}+1\right)+p^{\prime} \geq \frac{1}{4} k^{\left(\frac{\gamma}{2}+2 \delta_{0}\right) r_{1}-\delta_{*}-\delta} .
$$

Assume that (530) does not hold. Then, by (529)

$$
\sum_{m=1}^{p-p^{\prime}} d_{m} \geq \frac{1}{4} k^{\left(\frac{\gamma}{2}+2 \delta_{0}\right) r_{1}}
$$

since $D=k^{\left(\frac{\gamma}{2}+2 \delta_{0}\right) r_{1}}$. Obviously,

$$
d_{m} \leq n_{m} k^{\frac{\gamma r_{1}}{6}}
$$


where $n_{m}$ is the number of $\mathcal{M}^{(2)}$ points in the white cluster number $m, m=1, \ldots, p-p^{\prime}$. Let $\ell$ be the size of a minimal box containing all these white clusters. It is easy to see that

$$
\ell \leq k^{\delta} \sum_{q=1}^{p+1}\left(s_{q}+1\right)+k^{\delta_{*}+\delta} p^{\prime}+\sum_{m=1}^{p-p^{\prime}} d_{m} \leq \frac{1}{4} k^{\left(\frac{\gamma}{2}+2 \delta_{0}\right) r_{1}}+\sum_{m=1}^{p-p^{\prime}} d_{m} \leq 2 \sum_{m=1}^{p-p^{\prime}} d_{m} .
$$

Here we also used (531) and the inequality opposite to (530). By Lemma 4.18

$$
\sum_{m=1}^{p-p^{\prime}} n_{m} \leq C \ell^{2 / 3} k
$$

Combining inequalities (532)-(534) and solving for $\sum_{m=1}^{p-p^{\prime}} d_{m}$, we obtain: $\sum_{m=1}^{p-p^{\prime}} d_{m}<$ $k^{\frac{\gamma r_{1}}{2}+3}$. This contradicts to (531). Thus, we proved (530). Using (530) and the obvious inequality $\sum_{q=1}^{p+1}\left(s_{q}+1\right)+p^{\prime} \leq R+1$ proves the lemma.

\subsection{Appendix 7}

Lemma 10.9. Let $R$ be the smallest positive integer for which (310) holds in the case of a black cluster. We have $R>k^{\gamma r_{1}+\delta_{0} r_{1}-\delta_{*}-2 \delta}$.

Proof. We again use formula (528), where $P_{j_{q}}$ are projections on non-resonant, white and grey components in a component of a black region. Assume first that all components $\Pi_{j_{q}}$ can be placed in one $\left\||\cdot \||\right.$ box of the size $4 k^{\gamma r_{1}+\delta_{0} r_{1}}$. Obviously,

$$
\frac{D}{2} \leq k^{\delta} \sum_{q=1}^{p+1}\left(s_{q}+1\right)+k^{\delta_{*}+\delta} p^{\prime}+\sum_{m} d_{m}^{w}+\sum_{\tilde{m}} d_{\tilde{m}}^{g}
$$

where $\frac{D}{2}$ is the $\left\||\cdot \||\right.$ distance between the supports of $P^{\partial}$ and $P^{(i n t)}, p^{\prime}$ is the number of non-resonant components in the black component, $\sum_{m} d_{m}^{w}$ and $\sum_{\tilde{m}} d_{\tilde{m}}^{g}$ are the total lengths of white and grey components in the black component. Let us prove first that $k^{\delta} \sum\left(s_{q}+1\right)+k^{\delta_{*}+\delta} p^{\prime}+\sum_{m} d_{m}^{w}>\frac{1}{4} k^{\gamma r_{1}+\delta_{0} r_{1}}$. Suppose that it is not so. Then, by (535), $\sum_{\tilde{m}} d_{\tilde{m}}^{g}>\frac{1}{4} k^{\gamma r_{1}+\delta_{0} r_{1}}$, since $D=\frac{1}{2} k^{\gamma r_{1}+\delta_{0} r_{1}}$. The $4 k^{\gamma r_{1}+\delta_{0} r_{1}}$-box containing all components $\Pi_{j_{q}}$, consists of no more than $4^{4} k^{4 \delta_{0} r_{1}}$ boxes of the size $k^{\gamma r_{1}}$. Since all $\Pi_{j_{q}}$ are in white $k^{\gamma r_{1}}$-boxes, the total number of points of $\mathcal{M}^{(2)}$ in these white boxes does not exceed $c k^{\frac{1}{2} \gamma r_{1}+\delta_{0} r_{1}} \cdot k^{4 \delta_{0} r_{1}}$. Since each grey box contains more than $k^{\frac{1}{6} \gamma r_{1}-\delta_{0} r_{1}}$ points, the total number of grey boxes is less than $c k^{\frac{1}{2} \gamma r_{1}+\delta_{0} r_{1}} \cdot k^{4 \delta_{0} r_{1}} \cdot k^{-\frac{1}{6} \gamma r_{1}+\delta_{0} r_{1}}=c k^{\frac{1}{3} \gamma r_{1}+6 \delta_{0} r_{1}}$. Therefore, the total size of the grey region is less than $c k^{\frac{1}{3} \gamma r_{1}+6 \delta_{0} r_{1}} \cdot k^{\frac{1}{2} \gamma r_{1}+2 \delta_{0} r_{1}}=c k^{\frac{5}{6} \gamma r_{1}+8 \delta_{0} r_{1}}$, see the definition of a grey region. Since $\delta_{0}<\frac{1}{48} \gamma$, it is much less than $\frac{1}{4} k^{\gamma r_{1}+\delta_{0} r_{1}}$. We have arrived to the contradiction with the assumption $\sum_{\tilde{m}} d_{\tilde{m}}^{g}>\frac{1}{4} k^{\gamma r_{1}+\delta_{0} r_{1}}$. Therefore, 
$k^{\delta} \sum\left(s_{q}+1\right)+k^{\delta_{*}+\delta} p^{\prime}+\sum_{m} d_{m}^{w}>\frac{1}{4} k^{\gamma r_{1}+\delta_{0} r_{1}}$. Considering again that the total number of $\mathcal{M}^{(2)}$ points in the white boxes of the $4 k^{\gamma r_{1}+\delta_{0}}$-box does not exceed $c k^{\frac{\gamma r_{1}}{2}+5 \delta_{0} r_{1}}$, we obtain $\sum_{m} d_{m}^{w}<c k^{\frac{\gamma r_{1}}{2}+5 \delta_{0} r_{1}} \cdot 4 k^{\frac{\gamma r_{1}}{6}}<\frac{1}{20} k^{\gamma r_{1}+\delta_{0} r_{1}}$, see the definition of a white region. It follows $k^{\delta} \sum\left(s_{q}+1\right)+k^{\delta_{*}+\delta} p^{\prime}>\frac{1}{5} k^{\gamma r_{1}+\delta_{0} r_{1}}$. By construction, $R+1 \geq \sum\left(s_{q}+1\right)+p^{\prime}$. Therefore, $R>>k^{\gamma r_{1}+\delta_{0} r_{1}-\delta_{*}-2 \delta}$.

Assume that we cannot put all the components $\Pi_{j_{q}}$ in one $\||\cdot|||$-box of the size $4 k^{\gamma r_{1}+\delta_{0} r_{1}}$. Let us consider the box of this size around $\Pi_{j_{1}}$. Let $K$ be a number such that all $\Pi_{j_{q}}, q=1, \ldots K$ are completely in the box and $\Pi_{j_{K+1}}$ is not. Then, instead of (528) we consider just its piece

$$
\begin{aligned}
& P_{j_{1}} W\left(\hat{H}_{0}-k^{2}\right)^{-1}\left[\prod_{q=2}^{K}\left(P_{0} W\left(\hat{H}_{0}-k^{2}\right)^{-1}\right)^{s_{q}} P_{j_{q}} B P_{j_{q}} W\left(\hat{H}_{0}-k^{2}\right)^{-1}\right] \\
& \times\left(P_{0} W\left(\hat{H}_{0}-k^{2}\right)^{-1}\right)^{s_{K+1}} P_{j_{K+1}} .
\end{aligned}
$$

Further considerations are the same as in the previous case since by construction the distance between $\Pi_{j_{1}}$ and $\Pi_{j_{K+1}}$ is at least $\frac{1}{2} k^{\gamma r_{1}+\delta_{0} r_{1}}$.

\subsection{Appendix 8. On Application of Bezout Theorem}

Let $D(\vec{\varkappa}, \lambda)$ be the determinant of the truncated operator $H(\vec{\varkappa})-\lambda$ of the size $k^{r_{*}}, r_{*} \geq 1$. Obviously, $D$ is the polynomial of the degree $k^{4 r_{*}}$ with respect to $\varkappa_{1}, \varkappa_{2}$ and a line is not a solution of the equation $D(\vec{\varkappa}, \lambda)=0$. Let $\lambda$ be fixed, $\lambda=k^{2}$.

Definition 10.10. We call a piece of $D(\vec{\varkappa}, \lambda)=0$ elementary, if

1) it can be parameterized by $\varkappa_{1}: \varkappa_{2}=\varkappa_{2}\left(\varkappa_{1}\right)$ with $\left|\varkappa_{2}^{\prime}\left(\varkappa_{1}\right)\right| \leq 1$ or by $\varkappa_{2}: \varkappa_{1}=\varkappa_{1}\left(\varkappa_{2}\right)$ with $\left|\varkappa_{1}^{\prime}\left(\varkappa_{2}\right)\right| \leq 1$;

2) function $\varkappa_{1}=\varkappa_{1}\left(\varkappa_{2}\right)$ (or $\varkappa_{2}=\varkappa_{2}\left(\varkappa_{1}\right)$ ) is monotone and continuously differentiable;

3) it does not have inflection points inside;

4) it has a length not greater than 1.

We will show that the curve $D(\vec{\varkappa}, \lambda)=0$ can be split into elementary pieces and estimate the number of such pieces. In the proof we will apply several times the following statement (which is a simplified version of Bezout Theorem).

Theorem 10.11. Let $P$ and $Q$ be two plane real-valued polynomials of degree $p$ and $q$ respectively. If $P$ and $Q$ do not contain common factors then the total number of points satisfying $P(\vec{\varkappa})=0=Q(\vec{\varkappa})$ (i.e. number of points of intersection) does not exceed $p q$.

We have

Lemma 10.12. The set $D(\vec{\varkappa}, \lambda)=0$ can be split into $k^{17 r_{*}}$ or less elementary pieces. 
Proof. First, $D(\vec{\varkappa}, \lambda)$ can be represented as a product of simple (i.e. irreducible) factors (counting multiplicity). The total number of factors is less than $k^{4 r_{*}}$ which is also the bound for their total degree. We consider one of such simple factors $P$ and denote by $p$ its degree (note that we do ignore the multiplicity of the factor). Let us consider the points

$$
P(\vec{\varkappa})=0, \quad \frac{\partial}{\partial \varkappa_{2}} P(\vec{\varkappa})=0 .
$$

Since $P$ is irreducible and $\frac{\partial}{\partial \varkappa_{2}} P(\vec{\varkappa})$ has degree less than $p$ (we also notice that $\frac{\partial}{\partial \varkappa_{2}} P(\vec{\varkappa})$ is not identically zero since $D(\vec{\varkappa}, \lambda)=0$ does not contain lines) they do not have common factors. Thus, the number of such points $\vec{\varkappa}$ does not exceed $p(p-1)$. Next, by the same reasons the number of points

$$
P(\vec{\varkappa})=0, \quad \frac{\partial}{\partial \varkappa_{1}} P(\vec{\varkappa})=0
$$

does not exceed $p(p-1)$ and the number of points

$$
P(\vec{\varkappa})=0, \quad \frac{\partial P}{\partial \varkappa_{2}}(\vec{\varkappa})= \pm \frac{\partial P}{\partial \varkappa_{1}}(\vec{\varkappa})
$$

does not exceed $2 p(p-1)$. We split each previous piece by such points. Thus, we have at most $4 p(p-1)+1$ pieces, each end satisfying (537) or (539). The sign of $\left(\frac{\partial}{\partial \varkappa_{2}} P\right)^{2}-\left(\frac{\partial}{\partial \varkappa_{1}} P\right)^{2}$ is constant on each piece, i.e. the piece admits parametrization as in the property 2 of Definition 10.10, Making parametrization by $\varkappa_{1}$ or $\varkappa_{2}$, depending on the sign, we obtain that the length of a piece does not exceed $\sqrt{2} \cdot 4 k^{r_{*}}$ (obviously, $\left|\varkappa_{j}\right|<2 k^{r_{*}}$ ). Therefore the total length of the curve $P=0$ does not exceed $18 p^{2} k^{r_{*}}$. Next, for each piece where $\frac{\partial}{\partial \varkappa 2} P(\vec{\varkappa}) \neq 0$ inflection points of $P(\vec{\varkappa})=0$ are described by the system

$$
P=0, \quad P_{\varkappa_{2} \varkappa_{2}}\left(P_{\varkappa_{1}}\right)^{2}-2 P_{\varkappa_{1} \varkappa_{2}} P_{\varkappa_{1}} P_{\varkappa_{2}}+P_{\varkappa_{1} \varkappa_{1}}\left(P_{\varkappa_{2}}\right)^{2}=0
$$

Again, since $P$ is irreducible and no line is a solution, we have no common factors here and can apply Bezout Theorem. The number of points satisfying (540) does not exceed $p(2(p-1)+(p-2))=3 p^{2}-4 p$. Therefore, we have at most $12 p^{4}$ pieces with the ends satisfying (537) or (539) or (540). At last, we split each of these concave pieces into pieces with the length not greater than 1 . Considering that the total length of $P(\vec{\varkappa})=0$ is less that $18 p^{2} k^{r_{*}}$, we obtain that the total number of elementary pieces does not exceed $18 p^{2} k^{r_{*}}+12 p^{4}$. Taking the sum over all simple factors of $D$ we prove the lemma.

\subsection{Appendix 9. On the Proof of Geometric Lemmas Allowing to Deal with Clusters instead of Boxes}

In the proof of Lemma 5.14 it is important that we deal with the same curve generated by the determinant and just change the argument $\vec{\varkappa}$. At the same time, a priori we have the 
estimates for the resolvent of the operator reduced onto a particular cluster. The form of clusters can vary which formally changes the projector and thus the determinant and the curve. Here we explain how to deal with this situation. We will show that every cluster (white, grey or black) can be embedded into a box of the fixed size (depending on the color of the cluster) such that the estimate for the resolvent on this box is essentially the same as for the embedded cluster. We also notice that the estimate for the number of points of $\mathcal{M}^{(2)}$ inside these boxes is the same as the worst possible estimate for the corresponding cluster (see Lemmas 4.19, 4.20, 4.21). This justifies the application of Lemma 5.14 in the proof of Lemma 5.15 .

By construction, white clusters are separated from each other by the distance no less than $k^{\gamma r_{1} / 6}$. Grey and black clusters are separated by the distance at least $k^{\gamma r_{1} / 2+2 \delta_{0} r_{1}}$ and $k^{\gamma r_{1}+\delta_{0} r_{1}}$, respectively. Consider, first, a white cluster. Let $\Pi_{w}$ be a singular white cluster, namely,

$$
\left\|\left(P_{w}\left(H-k^{2}\right) P_{w}\right)^{-1}\right\|>k^{\xi}, \quad \xi>k^{\gamma r_{1} / 6-2 \delta_{*}},
$$

here and below $H=H\left(\vec{\varkappa}^{(2)}\left(\varphi_{0}\right)\right), P_{w}$ is the projector corresponding to $\Pi_{w}$. By construction, $\Pi_{w}$ belongs to a small white box and its neighbors. Let us refer to it as expanded small white box. Its size is $3 k^{\gamma r_{1} / 2+2 \delta_{0} r_{1}}$ and it contains less than $k^{\gamma r_{1} / 6-\delta_{0} r_{1}}$ elements of $\mathcal{M}^{(2)}$.

Lemma 10.13. If (541) holds for a white cluster $\Pi_{w}$ then

$$
\left\|\left(P\left(H-k^{2}\right) P\right)^{-1}\right\|>c k^{k^{\gamma r_{1} / 6-2 \delta_{*}}}
$$

$P$ being the projector corresponding to the expanded small white box $\Pi$ containing $\Pi_{w}$. The box $\Pi$ has the size $3 k^{\gamma r_{1} / 2+2 \delta_{0} r_{1}}$ and contains less than $k^{\gamma r_{1} / 6-\delta_{0} r_{1}}$ elements of $\mathcal{M}^{(2)}$.

Proof. Assume (541) holds, but (542) does not. Let $f \in P_{w} \ell^{2}$ be such that $\|f\|=1$, $P_{w}\left(H-k^{2}\right) f=o\left(k^{-\xi}\right), \xi=k^{\gamma r_{1} / 6-2 \delta_{*}}$. Let us define

$$
g:=f-\left(P\left(H-k^{2}\right) P\right)^{-1}\left(P-P_{w}\right) V f .
$$

Now we have:

$$
\begin{aligned}
& P\left(H-k^{2}\right) P g=P\left(H-k^{2}\right) f-\left(P-P_{w}\right) V f= \\
& P_{w}\left(H-k^{2}\right) f+\left(P-P_{w}\right)\left(H-k^{2}\right) f-\left(P-P_{w}\right) V f= \\
& P_{w}\left(H-k^{2}\right) P_{w} f+\left(P-P_{w}\right)\left(H_{0}-k^{2}\right) P_{w} f=P_{w}\left(H-k^{2}\right) P_{w} f=o\left(k^{-\xi}\right) .
\end{aligned}
$$

If we show that

$$
\left\|P_{w}\left(P\left(H-k^{2}\right) P\right)^{-1}\left(P-P_{w}\right) V f\right\|=o(1),
$$

which means $\|g\| \geq 1+o(1)$, then the lemma easily follows by the way of contradiction. Thus, it remains to prove (1544). Denote $\tilde{f}:=\left(P-P_{w}\right) V f$. Let $\tilde{H}_{w}^{(2)}$ be the operator 
consisting of $k^{\delta}$-clusters in $\Pi$. Namely, $\tilde{H}_{w}^{(2)}=\sum_{i} P_{i} H P_{i}, P_{i}$ being projectors onto $k^{\delta_{-}}$ clusters. Formally,

$$
\begin{aligned}
& \left(P\left(H-k^{2}\right) P\right)^{-1} \tilde{f}=\sum_{r=0}^{R_{0}}\left(\tilde{H}_{w}^{(2)}-k^{2}\right)^{-1}\left(-\left(H-\tilde{H}_{w}^{(2)}\right)\left(\tilde{H}_{w}^{(2)}-k^{2}\right)^{-1}\right)^{r} \tilde{f}+ \\
& \left(P\left(H-k^{2}\right) P\right)^{-1}\left(-\left(H-\tilde{H}_{w}^{(2)}\right)\left(\tilde{H}_{w}^{(2)}-k^{2}\right)^{-1}\right)^{R_{0}+1} \tilde{f}, \quad R_{0}=\left[k^{\gamma r_{1} / 6-\delta_{*}-\delta}\right]-1 .
\end{aligned}
$$

Some of $k^{\delta}$-clusters $P_{i} H P_{i}$ are strongly resonant. However, their distance to the boundary of any white cluster is greater than $k^{\gamma r_{1} / 6}$. Using this fact and considering as in the proof of (298), we obtain

$$
\left\|\left(-\left(\tilde{H}_{w}^{(2)}-k^{2}\right)^{-1}\left(H-\tilde{H}_{w}^{(2)}\right)\left(\tilde{H}_{w}^{(2)}-k^{2}\right)^{-1}\right)^{s} \tilde{f}\right\|<c k^{-s \delta_{*} / 8} \quad \text { when } \quad 2 s \leq R_{0}+1,
$$

since $(\tilde{f})_{\mathbf{m}} \neq 0$ only near the boundary of a white cluster. Hence, the right hand part of (545) is well defined. Now, substituting (546) into (545) and using the estimate opposite to (542) we estimate the last term in (545). Thus (cf. the proof of (295)), we have

$$
P_{w}\left(P\left(H-k^{2}\right) P\right)^{-1} \tilde{f}=P_{w}\left[\left(I+O\left(k^{-\delta_{*} / 8}\right)\right)\left(\tilde{H}_{w}^{(2)}-k^{2}\right)^{-1}+O\left(k^{-\delta_{*} / 8}\right)\right]\left(P-P_{w}\right) V P_{w} f
$$

Using the identity $P_{w}\left(\tilde{H}_{w}^{(2)}-k^{2}\right)^{-1}\left(P-P_{w}\right)=0$ we estimate

$$
\left\|P_{w}\left(P\left(H-k^{2}\right) P\right)^{-1} \tilde{f}\right\| \leq o(1)+O\left(k^{-\delta_{*} / 8}\right)\left\|\left(\tilde{H}_{w}^{(2)}-k^{2}\right)^{-1}\left(P-P_{w}\right) V P_{w}\right\| .
$$

We notice that near the boundary of $\Pi_{w}$ all clusters satisfy the estimate (261). Thus we can apply the constructions from the proof of Theorem 4.1 (see in particular the proof of (180) and especially the proof of (185), (186) and (188)). We get

$$
\left\|\left(\tilde{H}_{w}^{(2)}-k^{2}\right)^{-1}\left(P-P_{w}\right) V P_{w}\right\|=O\left(k^{214 \mu \delta}\right),
$$

which together with (547) proves (544).

For singular grey and black clusters the proof is very similar. So, we just introduce corresponding objects and formulate the results.

Let $\Pi_{g}$ be a singular grey cluster, i.e.

$$
\left\|\left(P_{g}\left(H-k^{2}\right) P_{g}\right)^{-1}\right\|>k^{\xi}, \quad \xi>k^{\gamma r_{1} / 2+2 \delta_{0} r_{1}-2 \delta_{*}},
$$

$P_{g}$ being the projector corresponding to $\Pi_{g}$. By construction, $\Pi_{g}$ belongs to a big white box and its neighbors. We refer to it as expanded big white box. Its size is $3 k^{\gamma r_{1}}$ and it contains less than $k^{\gamma r_{1} / 2+\delta_{0} r_{1}}$ elements of $\mathcal{M}^{(2)}$. 
Lemma 10.14. If (548) holds for a grey cluster $\Pi_{g}$, such that all the white clusters imbedded into it do not satisfy (542), then

$$
\left\|\left(P\left(H-k^{2}\right) P\right)^{-1}\right\|>c k^{k^{\gamma r_{1} / 2+2 \delta_{0} r_{1}-2 \delta_{*}}},
$$

$P$ being the projector corresponding to the expanded big white box $\Pi$ containing $\Pi_{g}$. The box $\Pi$ has the size $3 k^{\gamma r_{1}}$ and it contains less than $k^{\gamma r_{1} / 2+\delta_{0} r_{1}}$ elements of $\mathcal{M}^{(2)}$.

The proof is analogous to that of Lemma 10.13 up to the obvious changes: instead of $P_{w}$ we take $P_{g}, R_{0}=\left[k^{\frac{1}{2} \gamma r_{1}+2 \delta_{0} r_{1}-\delta_{*}-\delta}\right]-1$ and $\tilde{H}_{w}^{(2)}$ is replaced by $\tilde{H}_{g}^{(2)}$ which consists of $k^{\delta}$ non-resonance clusters and white clusters, which do not satisfy (542).

Let $\Pi_{b}$ be a singular black cluster, i.e.

$$
\left\|\left(P_{b}\left(H-k^{2}\right) P_{b}\right)^{-1}\right\|>k^{\xi}, \quad \xi>k^{\gamma r_{1}+\delta_{0} r_{1}-2 \delta_{*}},
$$

$P_{b}$ being the projector corresponding to $\Pi_{b}$. By Lemma 4.19 any black cluster can be covered by a box of the size $c k^{3 \gamma r_{1} / 2+3}$ containing less than $c k^{\gamma r_{1}+3}$ elements of $\mathcal{M}^{(2)}$. We refer to it as expanded black box.

Lemma 10.15. If (550) holds for a black cluster $\Pi_{b}$, such that all the white and grey clusters imbedded into it do not satisfy (1542), (1549), then

$$
\left\|\left(P\left(H-k^{2}\right) P\right)^{-1}\right\|>c k^{k^{\gamma r_{1}+\delta_{0} r_{1}-2 \delta_{*}}}
$$

$P$ being the projector corresponding to the expanded black box containing $\Pi_{b}$. The box $\Pi$ has the size $c k^{3 \gamma r_{1} / 2+3}$ and it contains less than $c k^{\gamma r_{1}+3}$ elements of $\mathcal{M}^{(2)}$.

\subsection{Appendix 10}

We consider $\vec{\varkappa}\left(\tau_{1}\right)=\mathbf{b}+\tau_{1} \mathbf{a}$. Let $b_{\mathbf{q}}=\left(\mathbf{b}+\vec{p}_{\mathbf{m}}, \vec{\nu}_{\mathbf{q}}\right), a_{\mathbf{q}}=\left(\mathbf{a}, \vec{\nu}_{\mathbf{q}}\right), t_{\mathbf{q}}=b_{\mathbf{q}}+a_{\mathbf{q}} \tau_{1}$ and $b_{\mathbf{q}}^{\perp}=\left(\mathbf{b}+\vec{p}_{\mathbf{m}}, \vec{\nu}_{\mathbf{q}}^{\perp}\right), a_{\mathbf{q}}^{\perp}=\left(\mathbf{a}, \vec{\nu}_{\mathbf{q}}^{\perp}\right), t_{\mathbf{q}}^{\perp}=b_{\mathbf{q}}^{\perp}+a_{\mathbf{q}}^{\perp} \tau_{1}, \mathbf{m}$ being the central point of $\mathcal{M}_{2}^{j, s}$. Let us consider the corresponding periodic operator $H_{1}^{\text {per }}\left(b_{\mathbf{q}}\right)$ associated with $\mathcal{M}_{2}^{j, s}$. Let $\mu \leq 6 C_{*}(V, \Lambda)($ see $(340))$. In our case

$$
\mu=k^{2}-\left(b_{\mathbf{q}}^{\perp}\right)^{2}=k^{2}-\left(t_{\mathbf{q}}^{\perp}\right)^{2}+\left(t_{\mathbf{q}}^{\perp}\right)^{2}-\left(b_{\mathbf{q}}^{\perp}\right)^{2} \leq \Lambda+5 C_{*} \leq 6 C_{*}
$$

by assumption. Let $\lambda_{n_{0}}\left(b_{\mathbf{q}}\right), \lambda_{n_{0}+1}\left(b_{\mathbf{q}}\right)$ be the eigenvalues of the operator being at the distance less than $\frac{1}{8} d$ from $\mu$, where $d$ is the length of the shortest zone of the operator $H_{1}^{\text {per }}$. If we have just one such eigenvalue or none, the consideration is analogous, just simpler. It is easy to see that $\lambda_{n_{0}}\left(t_{\mathbf{q}}\right), \lambda_{n_{0}+1}\left(t_{\mathbf{q}}\right)$ are holomorphic functions of $\tau_{1}$ in a neighborhood of zero, the size of the neighborhood depending only on $V, \Lambda$. The projection $E\left(\tau_{1}\right)$, corresponding to the pair of eigenvalues, can be extended as a holomorphic operator-function of $\tau_{1}$ in a similar neighborhood of zero and $\left\|E\left(\tau_{1}\right)\right\|<c(V, \Lambda)$ in this 
neighborhood. The resolvent $\left(H_{1}^{\text {per }}\left(t_{\mathbf{q}}\right)-\mu\right)^{-1}$ is a meromorphic function of $\tau_{1}$ for any fixed $\mu$ and $\left(H_{1}^{\text {per }}\left(t_{\mathbf{q}}\right)-\mu\right)^{-1}\left(I-E\left(\tau_{1}\right)\right)$ is a holomorphic function. The size of a neighborhood of zero where this holds, depends only on $V, \Lambda$ when $\mu<6 C_{*}$. Let $D_{0}\left(\tau_{1}\right)=\left(\lambda_{n_{0}}\left(t_{\mathbf{q}}\right)-\right.$ $\mu)\left(\lambda_{n_{0}+1}\left(t_{\mathbf{q}}\right)-\mu\right)$. Obviously, $\left.\left(H_{1}^{\text {per }}\left(t_{\mathbf{q}}\right)-\mu\right)^{-1} E\left(\tau_{1}\right)\right) D_{0}\left(\tau_{1}\right)$ is a holomorphic function bounded in norm by $C(V, \Lambda)$. Next, let $\mu_{1}\left(\tau_{1}\right)=\left(b_{\mathbf{q}}^{\perp}\right)^{2}-\left(t_{\mathbf{q}}^{\perp}\right)^{2}=-2 a_{\mathbf{q}}^{\perp} b_{\mathbf{q}}^{\perp} \tau_{1}-\left(a_{\mathbf{q}}^{\perp}\right)^{2} \tau_{1}^{2}$. Obviously, $\left(H_{1}^{\text {per }}\left(t_{\mathbf{q}}\right)-\mu-\mu_{1}\right)^{-1}$ is a meromorphic function of $\tau_{1}$. Perturbative arguments yield that $\left(H_{1}^{\text {per }}\left(t_{\mathbf{q}}\right)-\mu-\mu_{1}\right)^{-1}\left(I-E\left(\tau_{1}\right)\right)$ is a holomorphic function of $\tau_{1}$ in a sufficiently small neighborhood of the origin, the size of the neighborhood depending on $V, \Lambda$ only. This part of the resolvent is bounded by $C(V, \Lambda)$. Let $D_{1}\left(\tau_{1}\right)=\left(\lambda_{n_{0}}\left(t_{\mathbf{q}}\right)-\mu-\mu_{1}\right)\left(\lambda_{n_{0}+1}\left(t_{\mathbf{q}}\right)-\mu-\mu_{1}\right)$. Noting that $\left(H_{1}^{\text {per }}\left(t_{\mathbf{q}}\right)-\mu-\mu_{1}\right)^{-1} E\left(\tau_{1}\right) D_{1}\left(\tau_{1}\right)=\left(H_{1}^{\text {per }}\left(t_{\mathbf{q}}\right)-\mu\right)^{-1} E\left(\tau_{1}\right) D_{0}\left(\tau_{1}\right)-\mu_{1}\left(\tau_{1}\right) E\left(\tau_{1}\right)$, we obtain that

$$
\left\|\left(H_{1}^{p e r}\left(t_{\mathbf{q}}\right)-\mu-\mu_{1}\right)^{-1} E\left(\tau_{1}\right) D_{1}\left(\tau_{1}\right)\right\|<C(V, \Lambda)\left(1+\left|\mu_{1}\right|\right) .
$$

By assumption, $\left|\mu_{1}\right|<5 C_{*}(V, \Lambda)$. Hence,

$$
\left\|\left(H_{1}^{p e r}\left(t_{\mathbf{q}}\right)-\mu-\mu_{1}\right)^{-1}\right\|<\frac{C(V, \Lambda)}{D_{1}\left(\tau_{1}\right)} .
$$

The product $D_{1}\left(\tau_{1}\right)$ consists of two multipliers. Let us consider one of them: $\lambda_{n_{0}}\left(t_{\mathbf{q}}\right)-$ $\mu-\mu_{1}$. Using Taylor expansion of the third order for an eigenvalue, we obtain that $\lambda_{n_{0}}\left(t_{\mathbf{q}}\right)-\mu-\mu_{1}=P_{3}\left(\tau_{1}\right)+O\left(\tau_{1}^{4}\right)$, where $P_{3}$ is a polynomial of order three and $\left|O\left(\tau_{1}^{4}\right)\right|<$ $C(V, \Lambda) \tau_{1}^{4}$. By [43], $\left|\lambda_{n_{0}}^{\prime \prime}\left(t_{\mathbf{q}}\right)\right|+\left|\lambda_{n_{0}}^{\prime \prime \prime}\left(t_{\mathbf{q}}\right)\right|>c(V, \Lambda)>0$. Applying Rouché's Theorem, we obtain that $\lambda_{n_{0}}\left(t_{\mathbf{q}}\right)-\mu-\mu_{1}$ has no more than three zeros in a $r(V, \Lambda)$ - neighborhood of zero and $\left|\lambda_{n_{0}}\left(t_{\mathbf{q}}\right)-\mu-\mu_{1}\right|>c(V, \Lambda) \varepsilon^{3}$ at the distance $\varepsilon$ from the nearest zero, $\varepsilon<\varepsilon_{0}(V, \Lambda)$. Hence $\left|D_{1}\right|>c(V, \Lambda) \varepsilon^{6}$ and

$$
\left\|\left(H_{1}^{p e r}\left(t_{\mathbf{q}}\right)-\mu-\mu_{1}\right)^{-1}\right\|<C(V, \Lambda) \varepsilon^{-6} .
$$

\section{List of the main notations}

Here, for the sake of convenience, we provide the list of main notations and definitions with the directions where they are introduced in the text.

operator $H$ - formula (1)

$C, c$ are constants depending only on $V, C_{0}, c_{0}$ are absolute constants.

$Q$ and irrational number $\alpha$ - formula (2)

measure of irrationality $\mu$ - formulae (27), (28)

norm $\left\|\left|\vec{p}_{\mathbf{s}} \|\right|\right.$ - just before Lemma 2.1

$\delta, \Omega(\delta), \tilde{\Omega}(\delta)$ - at the beginning of Section 3.1

$\mathcal{O}_{\mathbf{m}}$ - formulae (58) and (72)

$\mathcal{M}, \tilde{\mathcal{M}}, \mathcal{M}_{1}, \tilde{\mathcal{M}}_{1}, \mathcal{M}_{2}, \tilde{\mathcal{M}}_{2}, \mathcal{M}_{2}^{j}, \tilde{\mathcal{M}}_{2}^{j}, \mathcal{M}^{\prime}$ - formula (74) and text below 
$\gamma$ - after the proof of Lemma 4.18

simple, black, grey, white and non-resonant regions - Subsection 4.4.1 (Step II) and Subsection 5.3.2 (Step III)

$\delta_{0}$ - see definition of the black regions in Subsection 4.4.1

$k_{*}$ - at the beginning of Section 5

$\beta$ and operator $\tilde{H}^{(2)}$ - at the beginning of Subsection 5.1

$r_{n}, r_{n}^{\prime}$ - relations (384), (363), (277), (278), Lemma 5.15, relations (165) and the beginning of Subsection 4.4 .1

operator $\tilde{H}^{(n)}$ - after Lemma 7.2, at the beginning of Subsections 7.3, 6.1, 5.1 and formula (166)

$\mathcal{M}^{(n)}$ - formulae (396), (344), (251)

$\mathcal{O}^{(n)}$ - formulae (404), (358), (271), (155), (157)

$\mathcal{W}^{(n)}$ - formulae (405), (359), (272), (156), (60)

$\omega^{(n)}$ - formulae (406), (360), (273), (157), (61)

$g_{r}^{(n)}$ - formulae (411), (365), (280), (168), (39)

$G_{r}^{(n)}$ - formulae (412), (366), (281), (169), (40)

contour $C_{n}$ - formulae (412), (366), Lemmas 4.30, 3.34 and formula (37)

$\lambda^{(n)}$ - formulae (413), (367), (282), (170), (42)

spectral projector $\mathcal{E}^{(n)}$ - formulae (415), (369), (284), (172), (45)

$d^{(n)}\left(\mathbf{s}, \mathbf{s}^{\prime}\right)$ - formulae (420), (374), (289), (177), (501)

$\varkappa^{(n)}$ and $h^{(n)}$ - Lemmas 7.21, 6.6, 5.5, 4.4, 3.11

$\mathcal{S}^{(n)}$ - formulae (392), (327), (205)

$\Omega_{s}^{(j)}\left(r_{n}\right)$ - formulae (395), (350), (345) and description of the simple region in Subsection 4.4 .1

\section{References}

[1] Yu. Karpeshina, R. Shterenberg, Multiscale analysis in momentum space for quasiperiodic potential in dimension two, J. Math. Physics 54 (2013), 7.

[2] E. I. Dinaburg, Ya. Sinai, The One-dimensional Schrödinger Equation with a Quasiperiodic Potential, Funct. Anal. Appl. 9 (1975), 279-289.

[3] H. Rûssmann, On the one dimensional Schrödinger equation with a quasi-periodic potential, Ann. N. Y. Acad. Sci. 357 (1980), 90-107.

[4] R. Johnson, J. Moser, The rotation number for almost periodic potentials, Commun. Math. Phys. 84 (1982), 403-438.

[5] J. Moser, J. Püschel, An extension of a result by Dinaburg and Sinai on quasiperiodic potentials, Comment. Math. Helvetic 59 (1984), 39-85. 
[6] H. L. Cycon, R. G. Froese, W. Kirsch, B. Simon, Schrödinger Operators, Berlin: Springer Verlag, 1987, corrected and extended 2nd printing, Springer Verlag, 2008.

[7] L. Pastur, A. Figotin, Spectra of Random and Almost-Periodic Operators, SpringerVerlag, 1992, 583 pp.

[8] L. H. Eliasson, Floquet Solutions for the One-dimensional Quasi-periodic Schrödinger Equation, Comm. Math. Phys. 146 (1992), no. 3, 447-482.

[9] S. Jitomirskaya, Metal-Insulator Transition for the Almost Mathieu Operator, Ann. of Math. 150 (1999), 1159-1175.

[10] B. Simon, Schrödinger Operators in the Twentieth Century, J. Math. Phys. 41 (2000), no. 6, 3523-3355.

[11] A. Fedotov, F. Klopp, On the Singular Spectrum for Adiabatic Quasi-periodic Schrödinger Operators on the real line, Ann. Henri Poincaré 5 (2004), no. 5, 929978.

[12] A. Fedotov, F. Klopp, On the Absolutely Continuous Spectrum of One-dimensional Quasi-periodic Schrödinger operators in the Adiabatic Limit, Trans. Amer. Math. Soc. 357 (2005), no. 11, 4481-4516.

[13] A. Fedotov, F. Klopp, Strong Resonant Tunneling, Level Repulsion and Spectral Type for One-dimensional Adiabatic Quasi-periodic Schrödinger Operators, Ann. Sci. École Norm. Sup. (4) 38 (2005), no. 6, 889-950.

[14] A. Avila, Global theory of one-frequency Schrodinger operators I: stratified analyticity of the Lyapunov exponent and the boundary of nonuniform hyperbolicity, arXiv:0905.3902.

[15] A. Avila, Global theory of one-frequency Schrodinger operators II: acriticality and finiteness of phase transitions for typical potentials, http://w3.impa.br/ avila/global2.pdf

[16] M. A. Shubin, Density of States for Selfadjoint Elliptic Operators with Almost Periodic Coefficients, Trudy sem. Petrovskii (Moscow University) 3 (1978), 243-281.

[17] J. Bellissard, R. Lima, and E. Scoppola, Localization in n-dimensional incommensurable structures, Commun. Math. Phys. 88, (1983) 465-477.

[18] A. L. Figotin, L. A. Pastur, An Exactly Solvable Model of a Multidimensional Incommensurate Structure, Commun. Math. Physics 95 (1984), 401-425.

[19] J. Bellissard, Almost periodicity in solid state Physics and $C^{*}$-algebras, Mat.-Fys. Medd danske Vid. Selsk. 42 (1989), no. 3, 35-75. 
[20] V. Chulaevsky, E. I. Dinaburg, Methods of KAM theory for Long-Range Quasiperiodic Potentials on $\mathbb{Z}^{\vec{\nu}}$. Pure Point Spectrum, Commun. Math. Physics 153 (1993), no. $3,559-577$.

[21] J. Bourgain, M. Goldstein, W. Schlag, Anderson Localization on $\mathbb{Z}^{2}$ with QuasiPeriodic Potential, Acta Math. 188 (2002), 41-87.

[22] J. Bourgain, On Quasi-Periodic Lattice Schrödinger Operators, Discrete and Continuous Dynamical Systems 10 (2004), no. 1\&2, 75-88.

[23] L. Parnovski, R. Shterenberg, Complete Asymptotic Expansion of the Integrated Density of States of Multidimensional Almost-periodic Schrödinger Operators, Ann. of Math. 176 (2012), no. 2, 1039-1096.

[24] S. Morozov, L. Parnovski, R. Shterenberg, Complete Asymptotic Expansion of the Integrated Density of States of Multidimensional Almost-periodic Pseudo-differential Operators, Ann. H. Poincaré 15 (2014), no. 2, 263-312.

[25] J. Bourgain, Anderson localization for quasi-periodic lattice Schrödinger operators on $\mathbb{Z}^{d}$, d arbitrary, Geom. Funct. Anal. 17 (2007), no. 3, 682-706.

[26] J. Bourgain, Green's function estimates for lattice Schrödinger operators and applications, Ann. of Math. Studies, 158, Princeton University Press, 2005.

[27] V. N. Popov, M. M. Skriganov, Remark on the Structure of the Spectrum of a Two-Dimensional Schrödinger Operator with Periodic Potential, Zap. Nauchn. Sem. Leningrad. Otdel. Mat. Inst. Steklov. (LOMI) 109 (1981), 131-133; English transl.: J. Soviet Math. 24 (1984), no. 2, 239-240.

[28] L.Parnovski, A.Sobolev, Bethe-Sommerfeld conjecture for periodic operators with strong perturbations, Invent. Math., 181(3) (2010), 467-540.

[29] M. M. Skriganov, A. V. Sobolev, On the Spectrum of a Limit-Periodic Schrödinger Operator, Algebra i Analiz, 17 (2005), no. 5; Engl. Transl.: St. Petersburg Math. J. 17 (2006), 815-833.

[30] Yu. Karpeshina, Y.-R. Lee, Spectral properties of polyharmonic operators with limitperiodic potential in dimension two, D'Analyse Mathématique 102 (2007), 225-310.

[31] Yu. Karpeshina, Y.-R. Lee, Absolutely Continuous Spectrum of a Polyharmonic Operator with a Limit Periodic Potential in Dimension Two, Communications in Partial Differential Equations 33 (2008), no. 9, 1711-1728.

[32] Yu. Karpeshina, Y.-R. Lee, Spectral properties of a limit-periodic Schrödinger operator in dimension two, J. Anal. Math. 120 (2013), 1-84. 
[33] G. Gallavotti, Perturbation Theory for Classical Hamiltonian Systems, in Scaling and Self-Similarity in Physics edited by J. Froehlich, Birkhäuser, Basel, Switzerland, 1983, 359-424.

[34] L.E. Thomas, S.R. Wassel, Stability of Hamiltonian systems at high evergy, J. Math. Phys. 33(10), (1992), 3367-3373.

[35] L. E. Thomas and S. R. Wassel, Semiclassical Operators at High Energy, Lecture Notes in Physics 403, edited by E. Balslev, Springer-Verlag, 1992, 194-223.

[36] J. Frölich, T. Spencer, Absence of Diffusion in the Anderson Tight Binding Model for Large Disorder and Low Energy, Commun. Math. Physics 88 (1983), no. 2, $151-184$.

[37] J. Bourgain, M. Goldstein, On Nonperturbative Localization with Quasi-Periodic Potential, Ann. of Math. 152 (2000), no. 3, 835-879.

[38] J. Bourgain, Quasiperiodic Solutions of Hamilton Perturbations of 2D Linear Schrödinger Equation, Ann. of Math. 148 (1998), no. 2, 363-439.

[39] T. Kato, Perturbation theory for linear operators, Springer-Verlag, Berlin, 1995.

[40] M. Reed, B. Simon, Methods of Modern Mathematical Physics, Vol IV, Academic Press, 3rd ed., New York-San Francisco-London (1987), 396 pp.

[41] A. Avila, S. Jitomirskaya, Almost Reducibility and Almost Localization, JEMS 12 (2010), no. 1, 93-131.

[42] J. Bourgain, S. Jitomirskaya, Absolutely Continuous Spectrum for 1D Quasiperiodic Operators, Invent. Math. 148 (2002), no. 3, 453-463.

[43] E. Korotyaev, Some properties of the quasimomentum of the one-dimensional Hill operator, (Russian) Zap. Nauchn. Sem. Leningrad. Otdel. Mat. Inst. Steklov. (LOMI) 195 (1991), Mat. Vopr. Teor. Rasprostr. Voln. 21, 48-57, 180; translation in J. Soviet Math. 62 (1992), no. 6, 3081-3087.

[44] J. Pöschel, Examples of discrete Schrödinger operators with pure point spectrum, Comm. Math. Phys. 88 (1983), 447-463.

[45] D. Damanik, Z. Gan, Limit-periodic Schrödinger operators on $\mathbb{Z}^{d}$ : Uniform localization, J. Func. Anal. 265 (2013), 435-448. 